\title{
Bosonic Spectral Function and the Electron-Phonon Interaction in HTSC Cuprates
}

\author{
E. G. Maksimov, ${ }^{1}$ M. L. Kulić, ${ }^{2,3}$ and O. V. Dolgov ${ }^{4}$ \\ ${ }^{1}$ I. E. Tamm Theoretical Department, Lebedev Physical Institute, 119991 Moscow, Russia \\ ${ }^{2}$ Institute for Theoretical Physics, Goethe University, 60438 Frankfurt am Main, Germany \\ ${ }^{3}$ Max-Born-Institut für Nichtlineare Optik und Kurzzeitspektroskopie, 12489 Berlin, Germany \\ ${ }^{4}$ Theoretische Abteilung, Max-Planck-Institut für Festkörperphysik, 70569 Stuttgart, Germany \\ Correspondence should be addressed to M. L. Kulić, kulic@th.physik.uni-frankfurt.de
}

Received 20 July 2009; Revised 1 November 2009; Accepted 24 February 2010

Academic Editor: Carlo Di Castro

Copyright ( $) 2010$ E. G. Maksimov et al. This is an open access article distributed under the Creative Commons Attribution License, which permits unrestricted use, distribution, and reproduction in any medium, provided the original work is properly cited.

\begin{abstract}
In this paper we discuss experimental evidence related to the structure and origin of the bosonic spectral function $\alpha^{2} F(\omega)$ in hightemperature superconducting (HTSC) cuprates at and near optimal doping. Global properties of $\alpha^{2} F(\omega)$, such as number and positions of peaks, are extracted by combining optics, neutron scattering, ARPES and tunnelling measurements. These methods give evidence for strong electron-phonon interaction (EPI) with $1<\lambda_{e p} \lesssim 3.5$ in cuprates near optimal doping. We clarify how these results are in favor of the modified Migdal-Eliashberg (ME) theory for HTSC cuprates near optimal doping. In Section 2 we discuss theoretical ingredients-such as strong EPI, strong correlations-which are necessary to explain the mechanism of d-wave pairing in optimally doped cuprates. These comprise the ME theory for EPI in strongly correlated systems which give rise to the forward scattering peak. The latter is supported by the long-range part of EPI due to the weakly screened Madelung interaction in the ionic-metallic structure of layered HTSC cuprates. In this approach EPI is responsible for the strength of pairing while the residual Coulomb interaction and spin fluctuations trigger the $\mathrm{d}$-wave pairing.
\end{abstract}

\section{Experimental Evidence for Strong EPI}

1.1. Introduction. In spite of an unprecedented intensive experimental and theoretical study after the discovery of high-temperature superconductivity (HTSC) in cuprates, there is, even twenty-three years after, no consensus on the pairing mechanism in these materials. At present there are two important experimental facts which are not under dispute: (1) the critical temperature $T_{c}$ in cuprates is high, with the maximum $T_{c}^{\max } \sim 160 \mathrm{~K}$ in the $\mathrm{Hg}-1223$ compounds; (2) the pairing in cuprates is $d$-wave like, that is, $\Delta(\mathbf{k}, \omega) \approx \Delta_{d}(\omega)\left(\cos k_{x}-\cos k_{y}\right)$. On the contrary there is a dispute concerning the scattering mechanism which governs normal state properties and pairing in cuprates. To this end, we stress that in the HTSC cuprates, a number of properties can be satisfactorily explained by assuming that the quasiparticle dynamics is governed by some electronboson scattering and in the superconducting state bosonic quasiparticles are responsible for Cooper pairing. Which bosonic quasiparticles are dominating in the cuprates is the subject which will be discussed in this work. It is known that the electron-boson (phonon) scattering is well described by the Migdal-Eliashberg theory if the adiabatic parameter $A \equiv \alpha \cdot \lambda\left(\omega_{B} / W_{b}\right)$ fulfills the condition $A \ll$ 1 , where $\lambda$ is the electron-boson coupling constant, $\omega_{B}$ is the characteristic bosonic energy, $W_{b}$ is the electronic band width, and $\alpha$ depends on numerical approximations $[1,2]$. The important characteristic of the electron-boson scattering is the Eliashberg spectral function $\alpha^{2} F\left(\mathbf{k}, \mathbf{k}^{\prime}, \omega\right)$ (or its average $\alpha^{2} F(\omega)$ ) which characterizes scattering of quasiparticle from $\mathbf{k}$ to $\mathbf{k}^{\prime}$ by exchanging bosonic energy $\omega$. Therefore, in systems with electron-boson scattering the knowledge of the spectral function is of crucial importance.

There are at least two approaches differing in assumed pairing bosons in the HTSC cuprates. The first one is based on the electron-phonon interaction (EPI), with the main proponents in [3-11], where mediating bosons are phonons and where the average spectral function $\alpha^{2} F(\omega)$ is similar 
to the phonon density of states $F_{\mathrm{ph}}(\omega)$. Note that $\alpha^{2} F(\omega)$ is not the product of two functions although sometimes one defines the function $\alpha^{2}(\omega)=\alpha^{2} F(\omega) / F(\omega)$ which should approximate the energy dependence of the strength of the EPI coupling. There are numerous experimental evidences in cuprates for the importance of the EPI scattering mechanism with a rather large coupling constant in the normal scattering channel $1<\lambda_{e p} \lesssim 3$, which will be discussed in detail below. In the EPI approach $\alpha^{2} F_{\mathrm{ph}}(\omega)$ is extracted from tunnelling measurements in conjunction with IR optical measurements. The HTSC cuprates are on the borderline and it is a natural question-under which condition can high $T_{c}$ be realized in the nonadiabatic limit $A \approx 1$ ?

The second approach [12-17] assumes that EPI is too weak to be responsible for high $T_{c}$ in cuprates and it is based on a phenomenological model for spin-fluctuation interaction $(S F I)$ as the dominating scattering mechanism, that is, it is a nonphononic mechanism. In this (phenomenological) approach the spectral function is proportional to the imaginary part of the spin susceptibility $\operatorname{Im} \chi\left(\mathbf{k}-\mathbf{k}^{\prime}, \omega\right)$, that is, $\alpha^{2} F\left(\mathbf{k}, \mathbf{k}^{\prime}, \omega\right) \sim g_{\text {sf }}^{2} \operatorname{Im} \chi\left(\mathbf{k}-\mathbf{k}^{\prime}, \omega\right)$ where $g_{\text {sf }}$ is the SFI coupling constant. NMR spectroscopy and magnetic neutron scattering give evidence that in HTSC cuprates $\chi(\mathbf{q}, \omega)$ is peaked at the antiferromagnetic wave vector $Q=$ $(\pi / a, \pi / a)$ and this property is favorable for $d$-wave pairing. The SFI theory roots basically on the strong electronic repulsion on $\mathrm{Cu}$ atoms, which is usually studied by the Hubbard model or its (more popular) derivative the $t$ - $J$ model. Regarding the possibility to explain high $T_{c}$ solely by strong correlations, as it is reviewed in [18], we stress two facts. First, at present there is no viable theory as well as experimental facts which can justify these (nonphononic) mechanisms of pairing with some exotic pairing mechanism such as RVB pairing [18], fractional statistics, anyon superconductivity, and so forth. Therefore we will not discuss these, in theoretical sense interesting approaches. Second, the central question in these nonphononic approaches is the following-do models based solely on the Hubbard Hamiltonian show up superconductivity at sufficiently high critical temperatures $\left(T_{c} \sim 100 \mathrm{~K}\right)$ ? Although the answer on this important question is not definitely settled, there are a number of numerical studies of these models which offer negative answers. For instance, the sign-free variational Monte Carlo algorithm in the $2 \mathrm{D}$ repulsive $(U>0)$ Hubbard model gives no evidence for superconductivity with high $T_{c}$, neither the BCS-like nor the Berezinskii-Kosterlitz-Thouless(BKT-) like [19]. At the same time, similar calculations show that there is a strong tendency to superconductivity in the attractive $(U<0)$ Hubbard model for the same strength of $U$, that is, at finite temperature in the $2 \mathrm{D}$ model with $U<0$ the BKT superconducting transition is favored. Concerning the possibility of HTSC in the $t-J$ model, various numerical calculations such as Monte Carlo calculations of the Drude spectral weight [20] and hightemperature expansion for the pairing susceptibility [21] give evidence that there is no superconductivity at temperatures characteristic for cuprates and if it exists $T_{c}$ must be rather low-few Kelvins. These numerical results tell us that the lack of high $T_{c}$ (even in $2 \mathrm{D}$ BKT phase) in the repulsive
$(U>0)$ single-band Hubbard model and in the $t$ - $J$ model is not only due to thermodynamical 2D-fluctuations (which at finite $T$ suppress and destroy superconducting phase coherence in large systems) but it is also mostly due to an inherent ineffectiveness of strong correlations to produce solely high $T_{c}$ in cuprates. These numerical results signal that the simple single-band Hubbard and its derivative the $t$ - $J$ model are insufficient to explain solely the pairing mechanism in cuprates and some additional ingredients must be included.

Since EPI is rather strong in cuprates, then it must be accounted for. As it will be argued in the following, the experimental support for the importance of EPI in cuprates comes from optics, tunnelling, and recent ARPES measurements $[22,23]$. It is worth mentioning that recent ARPES activity was a strong impetus for renewed experimental and theoretical studies of EPI in cuprates. However, in spite of accumulating experimental evidence for importance of EPI with $\lambda_{e p}>1$, there are occasionally reports which doubt its importance in cuprates. This is the case with recent interpretation of some optical measurements in terms of SFI only [24-27] and with the LDA-DFT (local density approximation-density functional theory) band-structure calculations $[28,29]$, where both claim that EPI is negligibly small, that is, $\lambda_{e p}<0.3$. The inappropriateness of these statements will be discussed in the following sections.

The paper is organized as follows. In Section 1 we will mainly discuss experimental results in cuprates at and near optimal doping by giving also minimal theoretical explanations which are related to the bosonic spectral function $\alpha^{2} F(\omega)$ as well as to the transport spectral function $\alpha_{\mathrm{tr}}^{2} F(\omega)$ and their relations to EPI. The reason that we study only cuprates at and near optimal doping is that in these systems there are rather well-defined quasiparticlesalthough strongly interacting - while in highly underdoped systems the superconductivity is perplexed and possibly masked by other phenomena, such as pseudogap effects, formation of small polarons, interaction with spin and (possibly charge) order parameters, pronounced inhomogeneities of the scattering centers, and so forth. As the ARPES experiments confirm, there are no polaronic effects in systems at and near the optimal doping, while there are pronounced polaronic effects due to EPI in undoped and very underdoped HTSC [8-11]. In this work we consider mainly those direct one-particle and two-particle probes of low-energy quasiparticle excitations and scattering rates which give information on the structure of the spectral functions $\alpha^{2} F\left(\mathbf{k}, \mathbf{k}^{\prime}, \omega\right)$ and $\alpha_{\mathrm{tr}}^{2} F(\omega)$ in systems near optimal doping. These are angle-resolved photoemission (ARPES), various arts of tunnelling spectroscopy such as superconductor/insulator/normal metal (SIN) junctions, break junctions, scanning-tunnelling microscope spectroscopy (STM), infrared $(I R)$ and Raman optics, inelastic neutron and Xray scattering, and so forth. We will argue that these direct probes give evidence for a rather strong EPI in cuprates. Some other experiments on EPI are also discussed in order to complete the arguments for the importance of EPI in cuprates. The detailed contents of Section 1 are the following. In Section 1.2 we discuss some prejudices related to the strength of EPI as well as on the Fermi-liquid behavior of 
HTSC cuprates. We argue that any nonphononic mechanism of pairing should have very large bare critical temperature $T_{c 0} \gg T_{c}$ in the presence of the large EPI coupling constant, $\lambda_{e p} \geq 1$, if the EPI spectral function is weakly momentum dependent, that is, if $\alpha^{2} F\left(\mathbf{k}, \mathbf{k}^{\prime}, \omega\right) \approx \alpha^{2} F(\omega)$ like in lowtemperature superconductors. The fact that EPI is large in the normal state of cuprates and the condition that it must be conform with $d$-wave pairing imply that EPI in HTSC cuprates should be strongly momentum dependent. In Section 1.3 we discuss direct and indirect experimental evidences for the importance of EPI in cuprates and for the weakness of SFI in cuprates. These are the following.

(a) Magnetic Neutron Scattering Measurements. These measurements provide dynamic spin susceptibility $\chi(\mathbf{q}, \omega)$ which is in the SFI phenomenological approach [1217] related to the Eliashberg spectral function, that is, $\alpha^{2} F_{\mathrm{sf}}\left(\mathbf{k}, \mathbf{k}^{\prime}, \omega\right) \sim g_{\text {sf }}^{2} \operatorname{Im} \chi\left(\mathbf{q}=\mathbf{k}-\mathbf{k}^{\prime}, \omega\right)$. We stress that such an approach can be theoretically justified only in the weak coupling limit, $g_{\text {sf }} \ll W_{b}$, where $W_{b}$ is the band width and $g_{\text {sf }}$ is the phenomenological SFI coupling constant. Here we discuss experimental results on YBCO which give evidence for strong rearrangement (with respect to $\omega$ ) of $\operatorname{Im} \chi(\mathbf{q}, \omega)$ (with $\mathbf{q}$ at and near $\mathbf{Q}=(\pi, \pi)$ ) by doping toward the optimal doped HTSC $[30,31]$. It turns out that in the optimally doped cuprates with $T_{c}=$ $92.5 \mathrm{~K} \operatorname{Im} \chi(\mathbf{Q}, \omega)$ is drastically suppressed compared to that in slightly underdoped ones with $T_{c}=91 \mathrm{~K}$. This fact implies that the SFI coupling constant $g_{\text {sf }}$ must be small.

(b) Optical Conductivity Measurements. From these measurements one can extract the transport relaxation rate $\gamma_{\operatorname{tr}}(\omega)$ and indirectly an approximative shape of the transport spectral function $\alpha_{\mathrm{tr}}^{2} F(\omega)$. In the case of systems near optimal doping we discuss the following questions. (i) First is the physical and quantitative difference between the optical relaxation rate $\gamma_{\operatorname{tr}}(\omega)$ and the quasiparticle relaxation rate $\gamma(\omega)$. It was shown in the past that equating these two (unequal) quantities is dangerous and brings incorrect results concerning the quasiparticle dynamics in most metals by including HTSC cuprates too [3-6, 32-38]. (ii) Second are methods of extraction of the transport spectral function $\alpha_{\mathrm{tr}}^{2} F(\omega)$. Although these methods give at finite temperature $T$ a blurred $\alpha_{\mathrm{tr}}^{2} F(\omega)$ which is (due to the ill-defined methods) temperature dependent, it turns out that the width and the shape of the extracted $\alpha_{\mathrm{tr}}^{2} F(\omega)$ are in favor of EPI. (iii) Third is the restricted sum rule for the optical weight as a function of $T$ which can be explained by strong EPI $[39,40]$. (iv) Fourth is the good agreement with experiments of the $T$ dependence of the resistivity $\rho(T)$ in optimally doped YBCO, where $\rho(T)$ is calculated by using the spectral function from tunnelling experiments. Recent femtosecond time-resolved optical spectroscopy in $\mathrm{La}_{2-x} \mathrm{Sr}_{x} \mathrm{CuO}_{4}$ which gives additional evidence for importance of EPI [41] will be shortly discussed.

(c) ARPES Measurements and EPI. From these measurements the self-energy $\Sigma(\mathbf{k}, \omega)$ is extracted as well as some properties of $\alpha^{2} F\left(\mathbf{k}, \mathbf{k}^{\prime}, \omega\right)$. Here we discuss the following items: (i) the existence of the nodal and antinodal kinks in optimally and slightly underdoped cuprates, as well as the structure of the ARPES self-energy $(\Sigma(\mathbf{k}, \omega))$ and its isotope dependence, which are all due to EPI; (ii) the appearance of different slopes of $\Sigma(\mathbf{k}, \omega)$ at low $\left(\omega \ll \omega_{\text {ph }}\right)$ and high energies $\left(\omega \gg \omega_{\mathrm{ph}}\right)$ which can be explained by the strong EPI; (iii) the formation of small polarons in the undoped HTSC which was interpreted to be due to strong EPI-this gives rise to phonon side bands which are clearly seen in ARPES of undoped HTSC $[10,11]$.

(d) Tunnelling Spectroscopy. It is well known that this method is of an immense importance in obtaining the spectral function $\alpha^{2} F(\omega)$ from tunnelling conductance. In this part we discuss the following items: (i) the extracted Eliashberg spectral function $\alpha^{2} F(\omega)$ with the coupling constant $\lambda^{(\text {tun })}=2-3.5$ from the tunnelling conductance of break-junctions in optimally doped YBCO and Bi-2212 [4255] which gives that the maxima of $\alpha^{2} F(\omega)$ coincide with the maxima in the phonon density of states $F_{\mathrm{ph}}(\omega)$; (ii) the existence of eleven peaks in $-d^{2} I / d V^{2}$ in superconducting $\mathrm{La}_{1.84} \mathrm{Sr}_{0.16} \mathrm{CuO}_{4}$ films [56], where these peaks match precisely with the peaks in the intensity of the existing phonon Raman scattering data [57]; (iii) the presence of the dip in $d I / d V$ in STM which shows the pronounced oxygen isotope effect and important role of these phonons.

(e) Inelastic Neutron and X-Ray Scattering Measurements. From these experiments one can extract the phonon density of state $F_{\mathrm{ph}}(\omega)$ and in some cases the strengths of the quasiparticle coupling with various phonon modes. These experiments give sufficient evidence for quantitative inadequacy of LDA-DFT calculations in HTSC cuprates. Here we argue that the large softening and broadening of the half-breathing $\mathrm{Cu}-\mathrm{O}$ bond-stretching phonon, of the apical oxygen phonons and of the oxygen $B_{1 g}$ buckling phonons (in LSCO, BSCO, YBCO), cannot be explained by LDA-DFT. It is curious that the magnitude of the softening can be partially obtained by LDA-DFT but the calculated widths of some important modes are an order of magnitude smaller than the neutron scattering data show. This remarkable fact confirms that additionally the inadequacy of LDA-DFT in strongly correlated systems and a more sophisticated manybody theory for EPI is needed. The problem of EPI will be discussed in more details in Section 2.

In Section 1.4 brief summary of Section 1 is given. Since we are dealing with the electron-boson scattering in cuprates near the optimal doping, then in Appendix A (and in Section 2) we introduce the reader briefly to the MigdalEliashberg theory for superconductors (and normal metals) where the quasiparticle spectral function $\alpha^{2} F\left(\mathbf{k}, \mathbf{k}^{\prime}, \omega\right)$ and the transport spectral function $\alpha_{\mathrm{tr}}^{2} F(\omega)$ are defined.

Finally, one can pose a question-do the experimental results of the above enumerated spectroscopic methods allow a building of a satisfactory and physically reasonable microscopic theory for basic scattering and pairing mechanism in cuprates? The posed question is very modest compared to the much stringent request for the theory of everythingwhich would be able to explain all properties of HTSC materials. Such an ambitious project is not realized even in those low-temperature conventional superconductors where it is definitely proved that in most materials the pairing is due to EPI and many properties are well accounted for by the Migdal-Eliashberg theory. For an illustration, let us mention only two examples. First, the experimental value 
for the coherence peak in the microwave response $\sigma_{s}(T<$ $T_{c}, \omega=$ const) at $\omega=17 \mathrm{GHz}$ in the superconducting $\mathrm{Nb}$ is much higher than the theoretical value obtained by the strong coupling Eliashberg theory [58]. So to say, the theory explains the coherence peak at $17 \mathrm{GHz}$ in $\mathrm{Nb}$ qualitatively but not quantitatively. However, the measurements at higher frequency $\omega \sim 60 \mathrm{GHz}$ are in agreement with the Eliashberg theory [59]. Then one can say that instead of the theory of everything we deal with a satisfactory theory, which allows us qualitative and in many aspects quantitative explanation of phenomena in superconducting state. Second example is the experimental boron (B) isotope effect in $\operatorname{MgB}_{2}\left(T_{c} \approx 40 \mathrm{~K}\right)$ which is smaller than the theoretical value, that is, $\alpha_{\mathrm{B}}^{\exp } \approx$ $0.3<\alpha_{\mathrm{B}}^{\text {th }}=0.5$, although the pairing is due to EPI for boron vibrations [60]. Since the theory of everything is impossible in the complex materials such as HTSC cuprates in Section 1, we will not discuss those phenomena which need much more microscopic details and/or more sophisticated many-body theory. These are selected by chance: (i) large ratio $2 \Delta / T_{c}$ which is on optimally doped YBCO and BSCO $\approx 5$ and 7 , respectively, while in underdoped $\mathrm{BSCO}$ one has even $\left(2 \Delta / T_{c}\right) \approx 20$; (ii) peculiarities of the coherence peak in the microwave response $\sigma(T)$ in HTSC cuprates, which is peaked at $T$ much smaller than $T_{c}$, contrary to the case of LTSC where it occurs near $T_{c}$; (iii) the dependence of $T_{c}$ on the number of $\mathrm{CuO}_{2}$ in the unit cell; (iv) the temperature dependence of the Hall coefficient; (v) distribution of states in the vortex core, and so forth.

The microscopic theory of the mechanism for superconducting pairing in HTSC cuprates will be discussed in Section 2. In Section 2.1 we introduce an $a b$ initio manybody theory of superconductivity which is based on the fundamental (microscopic) Hamiltonian and the manybody technique. This theory can in principle calculate measurable properties of materials such as the critical temperature $T_{c}$, the critical fields, the dynamic and transport properties, and so forth. However, although this method is in principle exact, which needs only some fundamental constants $e, \hbar, m_{e}, M_{\text {ion }}, k_{B}$ and the chemical composition of superconducting materials, it was practically never realized in practice due to the complexity of many-body interactions - electron-electron and electron-lattice-as well as of structural properties. Fortunately, the problem can be simplified by using the fact that superconductivity is a lowenergy phenomenon characterized by the very small energy parameters $\left(T_{c} / E_{F}, \Delta / E_{F}, \omega_{\mathrm{ph}} / E_{F}\right) \ll 1$. It turns out that one can integrate high-energy electronic processes (which are not changed by the appearance of superconductivity) and then solve the low-energy problem by the (so-called) strongcoupling Migdal-Eliashberg theory. It turns out that in such an approach the physics is separated into the following: (1) the solution of the ideal band-structure Hamiltonian with the nonlocal exact crystal potential (sometimes called the excitation potential) $V_{\mathrm{IBS}}\left(\mathbf{r}, \mathbf{r}^{\prime}\right)$ (IBS-the ideal band structure) which includes the static self-energy $\left(\Sigma_{c 0}^{(h)}\left(\mathbf{r}, \mathbf{r}^{\prime}, \omega=0\right)\right)$ due to high-energy electronic processes, that is, $V_{\mathrm{IBS}}\left(\mathbf{r}, \mathbf{r}^{\prime}\right)=$ $\left[V_{e-i}(\mathbf{r})+V_{H}(\mathbf{r})\right] \delta\left(\mathbf{r}-\mathbf{r}^{\prime}\right)+\Sigma_{c 0}^{(h)}\left(\mathbf{r}, \mathbf{r}^{\prime}, \omega=0\right)$, with $V_{e-i}$ and $V_{H}$ being the electron-ion and Hartree potential, respectively;
(2) solving the low-energy Eliashberg equations. However, the calculation of the (excited) potential $V_{\mathrm{IBS}}\left(\mathbf{r}, \mathbf{r}^{\prime}\right)$ and the real EPI coupling $g_{e p}\left(\mathbf{r}, \mathbf{r}^{\prime}\right)=\delta V_{\mathrm{IBS}}\left(\mathbf{r}, \mathbf{r}^{\prime}\right) / \delta \mathbf{R}_{n}$, which include high-energy many-body electronic processes-for instance, the large Hubbard $U$ effects-is extremely difficult at present, especially in strongly correlated systems such as HTSC cuprates. Due to this difficulty the calculations of the EPI coupling in the past were usually based on the LDADFT method which will be discussed in Section 2.2 in the contest of HTSC cuprates, where the nonlocal potential is replaced by the local potential $V_{\mathrm{LDA}}(\mathbf{r})$ - the ground-state potential-and the real EPI coupling by the "local" LDA one $g_{e p}(\mathbf{r})=\delta V_{\mathrm{LDA}}(\mathbf{r}) / \delta \mathbf{R}_{n}$. Since the exchange-correlation effects enter $V_{\mathrm{LDA}}(\mathbf{r})=V_{e-i}(\mathbf{r})+V_{H}(\mathbf{r})+V_{\mathrm{XC}}(\mathbf{r})$ via the local exchange-correlation potential $V_{\mathrm{XC}}(\mathbf{r})$, it is clear that the LDA-DFT method describes strong correlations scarcely and it is inadequate in HTSC cuprates (and other strongly correlated systems such as heavy fermions) where one needs an approach beyond the LDA-DFT method. In Section 2.3 we discuss a minimal theoretical model for HTSC cuprates which takes into account minimal number of electronic orbitals and strong correlations in a controllable manner [6]. This theory treats the interplay of EPI and strong correlations in systems with finite doping in a systematic and controllable way. The minimal model can be further reduced (in some range of parameters) to the single-band $t$-J model, which allows the approximative calculation of the excited potential $V_{\text {IBS }}\left(\mathbf{r}, \mathbf{r}^{\prime}\right)$ and the nonlocal EPI coupling $g_{e p}\left(\mathbf{r}, \mathbf{r}^{\prime}\right)$. As a result one obtains the momentum-dependent EPI coupling $g_{e p}\left(\mathbf{k}_{F}, \mathbf{q}\right)$ which is for small hole-doping $(\delta<0.3)$ strongly peaked at small transfer momenta-the forward scattering peak. In the framework of this minimal model it is possible to explain some important properties and resolve some puzzling experimental results, like the following, for instance. (a) Why is $d$-wave pairing realized in the presence of strong EPI? (b) Why is the transport coupling constant $\left(\lambda_{\mathrm{tr}}\right)$ rather smaller than the pairing one $\lambda$, that is, $\lambda_{\text {tr }} \lesssim \lambda / 3$ ? (c) Why is the mean-field (one-body) LDA-DFT approach unable to give reliable values for the EPI coupling constant in cuprates and how many-body effects can help? (d) Why is $d$-wave pairing robust in the presence of nonmagnetic impurities and defects? (e) Why are the ARPES nodal and antinodal kinks differently renormalized in the superconducting states, and so forth? In spite of the encouraging successes of this minimal model, at least in a qualitative explanation of numerous important properties of HTSC cuprates, we are at present stage rather far from a fully microscopic theory of HTSC cuprates which is able to explain high $T_{c}$. In that respect at the end of Section 2.3 we discuss possible improvements of the present minimal model in order to obtain at least a semiquantitative theory for HTSC cuprates.

Finally, we would like to point out that in real HTSC materials there are numerous experimental evidences for nanoscale inhomogeneities. For instance, recent STM experiments show rather large gap dispersion, at least on the surface of BSCO crystals [61-63], giving rise to a pronounced inhomogeneity of the superconducting order parameter $\Delta(\mathbf{k}, \mathbf{R})$, where $\mathbf{k}$ is the relative momentum of the Cooper 
pair and $\mathbf{R}$ is the center of mass of Cooper pairs. One possible reason for the inhomogeneity of $\Delta(\mathbf{k}, \mathbf{R})$ and disorder on the atomic scale can be due to extremely high doping level of $\sim(10-20) \%$ in HTSC cuprates which is many orders of magnitude larger than in standard semiconductors $\left(10^{21}\right.$ versus $10^{15}$ carrier concentration). There are some claims that high $T_{c}$ is exclusively due to these inhomogeneities (of an extrinsic or intrinsic origin) which may effectively increase pairing potential [64], while some others try to explain high $T_{c}$ solely within the inhomogeneous Hubbard or $t-J$ model. Here we will not discuss this interesting problem but mention only that the concept of $T_{c}$ increase by inhomogeneity is not well-defined, since the increase of $T_{c}$ is defined with respect to the average value $\bar{T}_{c}$. However, $\bar{T}_{c}$ is experimentally not well defined quantity and the hypothesis of an increase of $T_{c}$ by material inhomogeneities cannot be tested at all. In studying and analyzing HTSC cuprates near optimal doping we assume that basic effects are realized in nearly homogeneous systems and inhomogeneities are of secondary role, which deserve to be studied and discussed separately.

1.2. EPI versus Nonphononic Mechanisms. Concerning the high $T_{c}$ values in cuprates, two dilemmas have been dominating after its discovery: (i) which interaction is responsible for strong quasiparticle scattering in the normal state? This question is related also to the dilemma of Fermi versus non-Fermi liquid; (ii) What is the mediating (gluing) boson responsible for the superconducting pairing, that is, phonons or nonphonons? In the last twenty-three years, the scientific community was overwhelmed by numerous proposed pairing mechanisms, most of which are hardly verifiable in HTSC cuprates.

(1) Fermi versus Non-Fermi Liquid in Cuprates. After discovery of HTSC in cuprates there was a large amount of evidence on strong scattering of quasiparticles which contradicts the canonical (popular but narrow) definition of the Fermi liquid, thus giving rise to numerous proposals of the so called non-Fermi liquids, such as Luttinger liquid, RVB theory, marginal Fermi liquid, and so forth. In our opinion there is no need for these radical approaches in explaining basic physics in cuprates at least in optimally, slightly underdoped and overdoped metallic and superconducting HTSC cuprates. Here we give some clarifications related to the dilemma of Fermi versus non-Fermi liquid. The definition of the canonical Fermi liquid (based on the Landau work) in interacting Fermi systems comprises the following properties: (1) there are quasiparticles with charge $q= \pm e$, spin $s=1 / 2$, and low-energy excitations $\xi_{\mathrm{k}}\left(=\epsilon_{\mathrm{k}}-\mu\right)$ which are much larger than their inverse life-times, that is, $\xi_{\mathbf{k}} \gg 1 / \tau_{\mathbf{k}} \sim \xi_{\mathbf{k}}^{2} / W_{b}$. Since the level width $\Gamma=2 / \tau_{\mathbf{k}}$ of the quasiparticle is negligibly small, this means that the excited states of the Fermi liquid are placed in one-to-one correspondence with the excited states of the free Fermi gas; (2) at $T=0 \mathrm{~K}$ there is an energy level $\xi_{\mathbf{k}_{F}}=0$ which defines the Fermi surface on which the Fermi quasiparticle distribution function $n_{F}\left(\xi_{\mathbf{k}}\right)$ has finite jump at $k_{F} ;$ (3) the number of quasiparticles under the Fermi surface is equal to the total number of conduction particles (we omit here other valence and core electrons) - the Luttinger theorem; (4) the interactions between quasiparticles are characterized by the set of Landau parameters which describe the low-temperature thermodynamics and transport properties. Having this definition in mind one can say that if fermionic quasiparticles interact with some bosonic excitation, for instance, with phonons, and if the coupling is sufficiently strong, then the former are not described by the canonical Fermi liquid since at energies and temperatures of the order of the characteristic (Debye) temperature $k_{B} \Theta_{D}\left(\equiv \hbar \omega_{D}\right.$ ) (for the Debye spectrum $\left.\sim \Theta_{D} / 5\right)$, that is, for $\xi_{\mathrm{k}} \sim \Theta_{D}$, one has $\tau_{\mathbf{k}}^{-1} \gtrsim \xi_{\mathbf{k}}$ and the quasiparticle picture (in the sense of the Landau definition) is broken down. In that respect an electron-boson system can be classified as a noncanonical Fermi liquid for sufficiently strong electron-boson coupling. It is nowadays well known that, for instance, $\mathrm{Al}, \mathrm{Zn}$ are weak coupling systems since for $\xi_{\mathbf{k}} \sim \Theta_{D}$ one has $\tau_{\mathbf{k}}^{-1} \ll \xi_{\mathbf{k}}$ and they are well described by the Landau theory. However, in (the noncanonical) cases where for higher energies $\xi_{\mathrm{k}} \sim$ $\Theta_{D}$ one has $\tau_{\mathbf{k}}^{-1} \gtrsim \xi_{\mathbf{k}}$, the electron-phonon system is satisfactory described by the Migdal-Eliashberg theory and the Boltzmann theory, where thermodynamic and transport properties depend on the spectral function $\alpha^{2} F_{\mathrm{sf}}\left(\mathbf{k}, \mathbf{k}^{\prime}, \omega\right)$ and its higher momenta. Since in HTSC cuprates the electron-boson (phonon) coupling is strong and $T_{c}$ is large, then it is natural that in the normal state (at $T>T_{c}$ ) we deal with a strong interacting noncanonical Fermi liquid which is for modest nonadiabaticity parameter $A<1$ described by the Migdal-Eliashberg theory, at least qualitatively and semiquantitatively. In order to justify this statement we will in the following elucidate some properties in more details by studying optical, ARPES, tunnelling and other experiments in HTSC oxides.

(2) Is There Limit of the EPI Strength? In spite of the reached experimental evidence in favor of strong EPI in HTSC oxides, there was a disproportion in the research activity (especially theoretical) in the past, since the investigation of the SFI mechanism of pairing prevailed in the literature. This trend was partly due to an incorrect statement in $[65,66]$ on the possible upper limit of $T_{c}$ in the phonon mechanism of pairing. Since in the past we have discussed this problem thoroughly in numerous papers-for the recent one see [67] — we will outline here the main issue and results only.

It is well known that in an electron-ion crystal, besides the attractive EPI, there is also repulsive Coulomb interaction. In case of an isotropic and homogeneous system with weak quasiparticle interaction, the effective potential $V_{\text {eff }}(\mathbf{k}, \omega)$ in the leading approximation looks like as for two external charges $(e)$ embedded in the medium with the total longitudinal dielectric function $\varepsilon_{\mathrm{tot}}(\mathbf{k}, \omega)$ ( $\mathbf{k}$ is the momentum and $\omega$ is the frequency) $[68,69]$, that is,

$$
V_{\text {eff }}(\mathbf{k}, \omega)=\frac{V_{\text {ext }}(\mathbf{k})}{\varepsilon_{\text {tot }}(\mathbf{k}, \omega)}=\frac{4 \pi e^{2}}{k^{2} \varepsilon_{\text {tot }}(\mathbf{k}, \omega)} .
$$

In case of strong interaction between quasiparticles, the state of embedded quasiparticles changes significantly due 
to interaction with other quasiparticles, giving rise to $V_{\text {eff }}(\mathbf{k}, \omega) \neq 4 \pi e^{2} / k^{2} \varepsilon_{\text {tot }}(\mathbf{k}, \omega)$. In that case $V_{\text {eff }}$ depends on other (than $\varepsilon_{\text {tot }}(\mathbf{k}, \omega)$ ) response functions. However, in the case when (1) holds, that is, when the weak-coupling limit is realized, $T_{c}$ is given by $T_{c} \approx \bar{\omega} \exp \left(-1 /\left(\lambda_{e p}-\mu^{*}\right)\right)[68-$ 70]. Here, $\lambda_{e p}$ is the EPI coupling constant, $\bar{\omega}$ is an average phonon frequency, and $\mu^{*}$ is the Coulomb pseudopotential, $\mu^{*}=\mu /\left(1+\mu \ln E_{F} / \bar{\omega}\right) \quad\left(E_{F}\right.$ is the Fermi energy). The couplings $\lambda_{e p}$ and $\mu$ are expressed by $\varepsilon_{\text {tot }}(\mathbf{k}, \omega=0)$ :

$$
\begin{aligned}
\mu-\lambda_{e p} & =\left\langle N(0) V_{\mathrm{eff}}(\mathbf{k}, \omega=0)\right\rangle \\
& =N(0) \int_{0}^{2 k_{F}} \frac{k d k}{2 k_{F}^{2}} \frac{4 \pi e^{2}}{k^{2} \varepsilon_{\mathrm{tot}}(\mathbf{k}, \omega=0)},
\end{aligned}
$$

where $N(0)$ is the density of states at the Fermi surface and $k_{F}$ is the Fermi momentum-see more in [3-5]. In $[65,66]$ it was claimed that the lattice stability of the system with respect to the charge density wave formation implies the condition $\varepsilon_{\text {tot }}(\mathbf{k}, \omega=0)>1$ for all $\mathbf{k}$. If this were correct, then from (2) it would follow that $\mu>\lambda_{e p}$, which limits the maximal value of $T_{c}$ to the value $T_{c}^{\max } \approx$ $E_{F} \exp \left(-4-3 / \lambda_{e p}\right)$. In typical metals $E_{F}<(1-10) \mathrm{eV}$, and if one accepts the statement in $[65,66]$ that $\lambda_{e p} \leq \mu(\leq 0.5)$, one obtains $T_{c} \sim(1-10) \mathrm{K}$. The latter result, if it would be correct, means that EPI is ineffective in producing not only high- $T_{c}$ superconductivity but also low-temperature superconductivity (LTS with $T_{c} \lesssim 20 \mathrm{~K}$ ). However, this result is in conflict first of all with experimental results in LTSC, where in numerous systems one has $\mu \leq \lambda_{e p}$ and $\lambda_{e p}>1$. For instance, $\lambda_{e p} \approx 2.6$ is realized in $P b B i$ alloy which is definitely much higher than $\mu(<1)$, and so forth.

Moreover, the basic theory tells us that $\varepsilon_{\text {tot }}(\mathbf{k} \neq 0, \omega)$ is not the response function $[68,69]$ (contrary to the assumption in $[65,66])$. Namely, if a small external potential $\delta V_{\text {ext }}(\mathbf{k}, \omega)$ is applied to the system (of electrons and ions in solids), it induces screening by charges of the medium and the total potential is given by $\delta V_{\text {tot }}(\mathbf{k}, \omega)=$ $\delta V_{\text {ext }}(\mathbf{k}, \omega) / \varepsilon_{\text {tot }}(\mathbf{k}, \omega)$, which means that $1 / \varepsilon_{\text {tot }}(\mathbf{k}, \omega)$ is the response function. The latter obeys the Kramers-Kronig dispersion relation which implies the following stability condition $[68,69]$ :

$$
\frac{1}{\varepsilon_{\mathrm{tot}}(\mathbf{k}, \omega=0)}<1, \quad \mathbf{k} \neq 0,
$$

that is, either

$$
\varepsilon_{\text {tot }}(\mathbf{k} \neq 0, \omega=0)>1
$$

or

$$
\varepsilon_{\text {tot }}(\mathbf{k} \neq 0, \omega=0)<0
$$

This important theorem invalidates the restriction on the maximal value of $T_{c}$ in the EPI mechanism given in [65, $66]$. We stress that the condition $\varepsilon_{\text {tot }}(\mathbf{k} \neq 0, \omega=0)<$ 0 is not in conflict with the lattice stability at all. For instance, in inhomogeneous systems such as crystal, the total longitudinal dielectric function is matrix in the space of reciprocal lattice vectors $(\mathbf{Q})$, that is, $\hat{\varepsilon}_{\text {tot }}(\mathbf{k}+\mathbf{Q}, \mathbf{k}+$
$\left.\mathbf{Q}^{\prime}, \omega\right)$, and $\varepsilon_{\text {tot }}(\mathbf{k}, \omega)$ is defined by $\varepsilon_{\text {tot }}^{-1}(\mathbf{k}, \omega)=\widehat{\varepsilon}_{\text {tot }}^{-1}(\mathbf{k}+$ $\mathbf{0}, \mathbf{k}+\mathbf{0}, \omega)$. In dense metallic systems with one ion per cell (such as metallic hydrogen) and with the electronic dielectric function $\varepsilon_{\mathrm{el}}(\mathbf{k}, 0)$, the macroscopic total dielectric function $\varepsilon_{\text {tot }}(\mathbf{k}, 0)$ is given by [71-73]

$$
\varepsilon_{\mathrm{tot}}(\mathbf{k}, 0)=\frac{\varepsilon_{\mathrm{el}}(\mathbf{k}, 0)}{1-1 / \varepsilon_{\mathrm{el}}(\mathbf{k}, 0) G_{e p}(\mathbf{k})} .
$$

At the same time the energy of the longitudinal phonon $\omega_{l}(\mathbf{k})$ is given by

$$
\omega_{l}^{2}(\mathbf{k})=\frac{\Omega_{p}^{2}}{\varepsilon_{\mathrm{el}}(\mathbf{k}, 0)}\left[1-\varepsilon_{\mathrm{el}}(\mathbf{k}, 0) G_{e p}(\mathbf{k})\right],
$$

where $\Omega_{p}^{2}$ is the ionic plasma frequency, and $G_{e p}$ is the local (electric) field correction-see [71-73]. The right condition for lattice stability requires that $\omega_{l}^{2}(\mathbf{k})>0$, which implies that for $\varepsilon_{\mathrm{el}}(\mathbf{k}, 0)>0$ one has $\varepsilon_{\mathrm{el}}(\mathbf{k}, 0) G_{e p}(\mathbf{k})<1$. The latter condition gives automatically $\varepsilon_{\text {tot }}(\mathbf{k}, 0)<0$. Furthermore, the calculations [71-73] show that in the metallic hydrogen $(\mathrm{H})$ crystal $\varepsilon_{\text {tot }}(\mathbf{k}, 0)<0$ for all $\mathbf{k} \neq \mathbf{0}$. Note that in metallic $H$ the EPI coupling constant is very large, that is, $\lambda_{e p} \approx 7$ and $T_{c}$ may reach very large value $T_{c} \approx 600 \mathrm{~K}$ [74]. Moreover, the analyses of crystals with more ions per unit cell [71-73] give that $\varepsilon_{\text {tot }}(\mathbf{k} \neq \mathbf{0}, 0)<0$ is more a rule than an exception-see Figure 1. The physical reason for $\varepsilon_{\text {tot }}(\mathbf{k} \neq \mathbf{0}, 0)<0$ is local field effects described by $G_{e p}(\mathbf{k})$. Whenever the local electric field $\mathbf{E}_{\text {loc }}$ acting on electrons (and ions) is different from the average electric field $\mathbf{E}$, that is, $\mathbf{E}_{\mathrm{loc}} \neq \mathbf{E}$, there are corrections to $\varepsilon_{\text {tot }}(\mathbf{k}, 0)$ which may lead to $\varepsilon_{\text {tot }}(\mathbf{k}, 0)<0$.

The above analysis tells us that in real crystals $\mathcal{E}_{\text {tot }}(\mathbf{k}, 0)$ can be negative in the large portion of the Brillouin zone thus giving rise to $\lambda_{e p}-\mu>0$ in (2). This means that analytic properties of the dielectric function $\varepsilon_{\text {tot }}(\mathbf{k}, \omega)$ do not limit $T_{c}$ in the phonon mechanism of pairing. This result does not mean that there is no limit on $T_{c}$ at all. We mention in advance that the local field effects play important role in HTSC cuprates, due to their layered structure with very unusual ionic-metallic binding, thus opening a possibility for large EPI.

In conclusion, we point out that there are no serious theoretical and experimental arguments for ignoring EPI in HTSC cuprates. To this end it is necessary to answer several important questions which are related to experimental findings in HTSC cuprates. (1) If EPI is important for pairing in HTSC cuprates and if superconductivity is of $d$-wave type, how are these two facts compatible? (2) Why is the transport EPI coupling constant $\lambda_{\text {tr }}$ (entering resistivity) rather smaller than the pairing EPI coupling constant $\lambda_{e p}(>1)$ (entering $T_{c}$ ), that is, why one has $\lambda_{\text {tr }}(\approx 0.6-1.4) \ll \lambda_{e p}(\sim 2-3.5)$ ? (3) If EPI is ineffective for pairing in HTSC oxides, in spite of $\lambda_{e p}>1$, why is it so?

(3) Is a Nonphononic Pairing Realized in HTSC? Regarding EPI one can pose a question about whether it contributes significantly to $d$-wave pairing in cuprates. Surprisingly, despite numerous experiments in favor of EPI, there is a belief that EPI is irrelevant for pairing [12-17]. This belief is mainly 


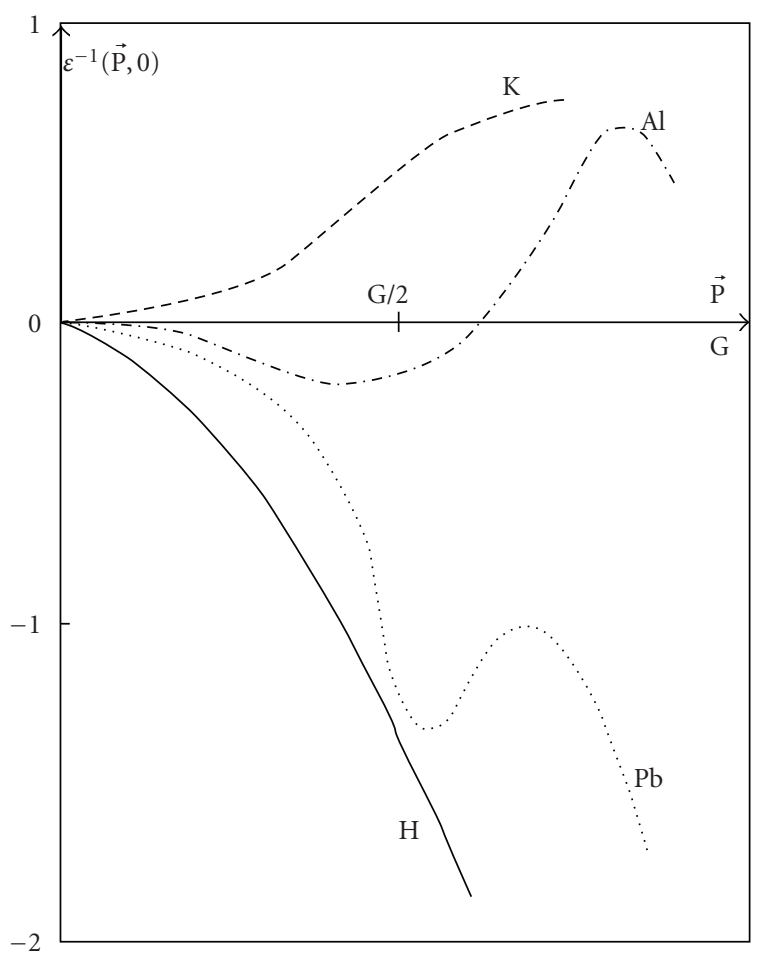

FIGURE 1: Inverse total static dielectric function $\varepsilon^{-1}(\mathbf{p})$ for normal metals $(\mathrm{K}, \mathrm{Al}, \mathrm{Pb})$ and metallic $\mathrm{H}$ in $\mathbf{p}=(1,0,0)$ direction. $\mathbf{G}$ is the reciprocal lattice vector.

based, first, on the above discussed incorrect lattice stability criterion related to the sign of $\varepsilon_{\text {tot }}(\mathbf{k}, 0)$, which implies small EPI and, second, on the well-established experimental fact that $d$-wave pairing is realized in cuprates [75], which is believed to be incompatible with EPI. Having in mind that EPI in HTSC at and near optimal doping is strong with $2<\lambda_{e p}<3.5$ (see below), we assume for the moment that the leading pairing mechanism in cuprates, which gives $d$-wave pairing, is due to some nonphononic mechanism. For instance, let us assume an exitonic mechanism due to the high-energy pairing boson $\left(\Omega_{\mathrm{nph}} \gg \omega_{\mathrm{ph}}\right)$ and with the bare critical temperature $T_{c 0}$ and look for the effect of EPI on $T_{c}$. If EPI is approximately isotropic, like in most LTSC materials, then it would be very detrimental for $d$ wave pairing. In the case of dominating isotropic EPI in the normal state and the exitonic-like pairing, then near $T_{c}$ the linearized Eliashberg equations have an approximative form for a weak nonphonon interaction (with the large characteristic frequency $\Omega_{\text {nph }}$ )

$$
\begin{gathered}
Z\left(\omega_{n}\right) \Delta_{n}(\mathbf{k}) \approx \pi T_{c} \sum_{m}^{\Omega_{\mathrm{nph}}} \sum_{\mathbf{q}} V_{\mathrm{nph}}(\mathbf{k}, \mathbf{q}, n, m) \frac{\Delta_{m}(\mathbf{q})}{\left|\omega_{m}\right|}, \\
Z\left(\omega_{n}\right) \approx 1+\frac{\Gamma_{e p}}{\omega_{n}} .
\end{gathered}
$$

For pure $d$-wave pairing with the pairing potential $V_{\text {nph }}=V_{\text {nph }}\left(\theta_{\mathbf{k}}, \theta_{\mathbf{q}}\right) \cdot \Theta\left(\Omega_{\text {nph }}-\left|\omega_{n}\right|\right) \Theta\left(\Omega_{\text {nph }}-\left|\omega_{n^{\prime}}\right|\right)$ with $V_{\mathrm{nph}}(\mathbf{k}, \mathbf{q})=V_{0} \cdot Y_{d}\left(\theta_{\mathbf{k}}\right) Y_{d}\left(\theta_{\mathbf{q}}\right)$ and $Y_{d}\left(\theta_{\mathbf{k}}\right)=\pi^{-1 / 2} \cos 2 \theta_{\mathbf{k}}$, one obtains $\Delta_{n}(\mathbf{k})=\Delta_{d} \cdot \Theta\left(\Omega_{\mathrm{nph}}-\left|\omega_{n}\right|\right) Y_{d}\left(\theta_{\mathbf{k}}\right)$ and the equation for $T_{c}$ - see [3-5]

$$
\ln \frac{T_{c}}{T_{c 0}} \approx \Psi\left(\frac{1}{2}\right)-\Psi\left(\frac{1}{2}+\frac{\Gamma_{e p}}{2 \pi T_{c}}\right) .
$$

Here $\Psi$ is the di-gamma function. At temperatures near $T_{c}$ one has $\Gamma_{e p} \approx 2 \pi \lambda_{e p} T_{c}$ and the solution of (9) is approximately $T_{c} \approx T_{c 0} \exp \left\{-\lambda_{e p}\right\}$ with $T_{c 0} \approx \Omega_{\text {nph }} \exp \left\{-\lambda_{\text {nph }}\right\}$, $\lambda_{\text {nph }}=N(0) V_{0}$. This means that for $T_{c}^{\max } \sim 160 \mathrm{~K}$ and $\lambda_{e p}>1$ the bare $T_{c 0}$ due to the nonphononic interaction must be very large, that is, $T_{c 0}>500 \mathrm{~K}$.

Concerning other nonphononic mechanisms, such as the SFI one, the effect of EPI in the framework of Eliashberg equations was studied numerically in [76]. The latter is based on (A.1) in Appendix A with the kernels in the normal and superconducting channels $\lambda_{\mathrm{kp}}^{Z}\left(i \nu_{n}\right)$ and $\lambda_{\mathrm{kp}}^{\Delta}$, respectively. Usually, the spin-fluctuation kernel $\lambda_{\mathrm{sf}, \mathbf{k p}}\left(i \nu_{n}\right)$ is taken in the FLEX approximation [77]. The calculations [76] confirm the very detrimental effect of the isotropic ( $\mathbf{k}$-independent) EPI on $d$-wave pairing due to SFI. For the bare SFI critical temperature $T_{c 0} \sim 100 \mathrm{~K}$ and for $\lambda_{e p}>1$ the calculations give very small (renormalized) critical temperature $T_{c} \ll 100 \mathrm{~K}$. These results tell us that a more realistic pairing interaction must be operative in cuprates and that EPI must be strongly momentum dependent and peaked at small transfer momenta [78-80]. Only in that case does strong EPI conform with $d$ wave pairing, either as its main cause or as a supporter of a nonphononic mechanism. In Section 2 we will argue that the strongly momentum-dependent EPI is important scattering mechanism in cuprates providing the strength of the pairing mechanism, while the residual Coulomb interaction (by including weaker SFI) triggers it to $d$-wave pairing.

1.3. Experimental Evidence for Strong EPI. In the following we discuss some important experiments which give evidence for strong electron-phonon interaction (EPI) in cuprates. However, before doing it, we will discuss some indicative inelastic magnetic neutron scattering (IMNS) measurements in cuprates whose results in fact seriously doubt in the effectiveness of the phenomenological SFI mechanism of pairing which is advocated in $[12-17,81]$. First, the experimental results related to the pronounced imaginary part of the susceptibility $\operatorname{Im} \chi\left(\mathbf{k}, k_{z}, \omega\right)$ in the normal state at and near the AF wave vector $\mathbf{k}=\mathbf{Q}=(\pi, \pi)$ were interpreted in a number of papers as a support for the SFI mechanism for pairing $[12-17,81]$. Second, the existence of the so called magnetic resonance peak of $\operatorname{Im} \chi\left(\mathbf{k}, k_{z}, \omega\right)$ (at some energies $\omega<2 \Delta$ ) in the superconducting state was also interpreted in a number of papers either as the origin of superconductivity or as a mechanism strongly affecting superconducting gap at the antinodal point.

\subsubsection{Magnetic Neutron Scattering and the Spin-Fluctuation Spectral Function}

(a) Huge Rearrangement of the SFI Spectral Function and Small Change of $T_{c}$. Before discussing experimental results in cuprates on the imaginary part of the spin susceptibility 
$\operatorname{Im} \chi(\mathbf{k}, \omega)$ we point out that in the (phenomenological) theories based on the spin-fluctuation interaction (SFI) the quasiparticle self-energy $\hat{\Sigma}_{\mathrm{sf}}\left(\mathbf{k}, \omega_{n}\right) \quad\left(\omega_{n}\right.$ is the Matsubara frequency and $\hat{\tau}_{0}$ is the Nambu matrix) in the normal and superconducting state and the effective (repulsive) pairing potential $V_{\mathrm{sf}}(\mathbf{k}, \omega)$ (where $i \omega_{n} \rightarrow \omega+i \eta$ ) are assumed in the form [12-17]

$$
\begin{gathered}
\hat{\Sigma}_{\mathrm{sf}}\left(\mathbf{k}, \omega_{n}\right)=\frac{T}{N} \sum_{\mathbf{k}^{\prime}, m} V_{\mathrm{sf}}\left(\mathbf{k}-\mathbf{k}^{\prime}, \omega_{n m}^{-}\right) \hat{\tau_{0}} \hat{G}\left(\mathbf{k}^{\prime}, \omega_{m}\right) \hat{\tau}_{0}, \\
V_{\mathrm{sf}}\left(\mathbf{k}, \omega_{n m}^{-}\right)=g_{\mathrm{sf}}^{2} \int_{-\infty}^{\infty} \frac{d \nu}{\pi} \frac{\operatorname{Im} \chi\left(\mathbf{q}, \nu+i 0^{+}\right)}{\nu-i \omega_{n m}^{-}},
\end{gathered}
$$

where $\omega_{n m}^{-} \equiv \omega_{n}-\omega_{m}$. Although the form of $V_{\text {sf }}$ cannot be justified theoretically, except in the weak coupling limit $\left(g_{\text {sf }} \ll W_{b}\right)$ only, it is often used in the analysis of the quasiparticle properties in the normal and superconducting state of cuprates where the spin susceptibility (spectral function) $\operatorname{Im} \chi(\mathbf{q}, \omega)$ is strongly peaked at and near the AF wave vector $\mathbf{Q}=(\pi / a, \pi / a)$.

Can the pairing mechanism in HTSC cuprates be explained by such a phenomenology and what is the prise for it is? The best answer is to look at the experimental results related to the inelastic magnetic neutron scattering (IMNS) which gives $\operatorname{Im} \chi(\mathbf{q}, \omega)$. In that respect very indicative and impressive IMNS measurements on $\mathrm{YBa}_{2} \mathrm{Cu}_{3} \mathrm{O}_{6+x}$, which are done by Bourges group [30], demonstrate that the normalstate susceptibility $\operatorname{Im} \chi^{\text {(odd) }}(\mathbf{q}, \omega)$ (the odd part of the spin susceptibility in the bilayer system) at $\mathbf{q}=\mathbf{Q}=(\pi, \pi)$ is strongly dependent on the hole-doping as it is shown in Figure 2.

The most pronounced result for our discussion is that by varying doping there is a huge rearrangement of $\operatorname{Im} \chi^{(\text {odd })}(\mathbf{Q}, \omega)$ in the normal state, especially in the energy (frequency) region which might be important for superconducting pairing, let us say $0 \mathrm{meV}<\omega<60 \mathrm{meV}$. This is clearly seen in the last two curves in Figure 2 where this rearrangement is very pronounced, while at the same time there is only small variation of the critical temperature $T_{c}$. It is seen in Figure 2 that in the underdoped $\mathrm{YBa}_{2} \mathrm{Cu}_{3} \mathrm{O}_{6.92}$ crystal $\operatorname{Im} \chi^{(\text {odd })}(\mathbf{Q}, \omega)$ and $S(\mathbf{Q})=N(0) g_{\text {sf }}^{2} \int_{0}^{60} d \omega \operatorname{Im} \chi^{(\text {odd })}(\mathbf{Q}, \omega)$ are much larger than that in the near optimally doped $\mathrm{YBa}_{2} \mathrm{Cu}_{3} \mathrm{O}_{6.97}$, that is, one has $S_{6.92}(\mathbf{Q}) \gg S_{6.97}(\mathbf{Q})$, although the difference in the corresponding critical temperatures $T_{c}$ is very small, that is, $T_{c}^{(6.92)}=91 \mathrm{~K}$ (in $\mathrm{YBa}_{2} \mathrm{Cu}_{3} \mathrm{O}_{6.92}$ ) and $T_{c}^{(6.97)}=92.5 \mathrm{~K}$ (in $\mathrm{YBa}_{2} \mathrm{Cu}_{3} \mathrm{O}_{6.97}$ ). This pronounced rearrangement and suppression of $\operatorname{Im} \chi^{\text {(odd) }}(\mathbf{Q}, \omega)$ in the normal state of YBCO by doping (toward the optimal doping) but with the negligible change in $T_{c}$ is strong evidence that the SFI pairing mechanism is not the dominating one in HTSC cuprates. This insensitivity of $T_{c}$, if interpreted in terms of the SFI coupling constant $\lambda_{\text {sf }}\left(\sim g_{\text {sf }}^{2}\right)$, means that the latter is small, that is, $\lambda_{\text {sf }}^{(\exp )} \ll 1$. We stress that the explanation of high $T_{c}$ in cuprates by the SFI phenomenological theory [12-17] assumes very large SFI coupling energy with $g_{\text {sf }}^{\text {(th) }} \approx 0.7 \mathrm{eV}$ while the frequency (energy) dependence of $\operatorname{Im} \chi(\mathbf{Q}, \omega)$ is extracted from the fit

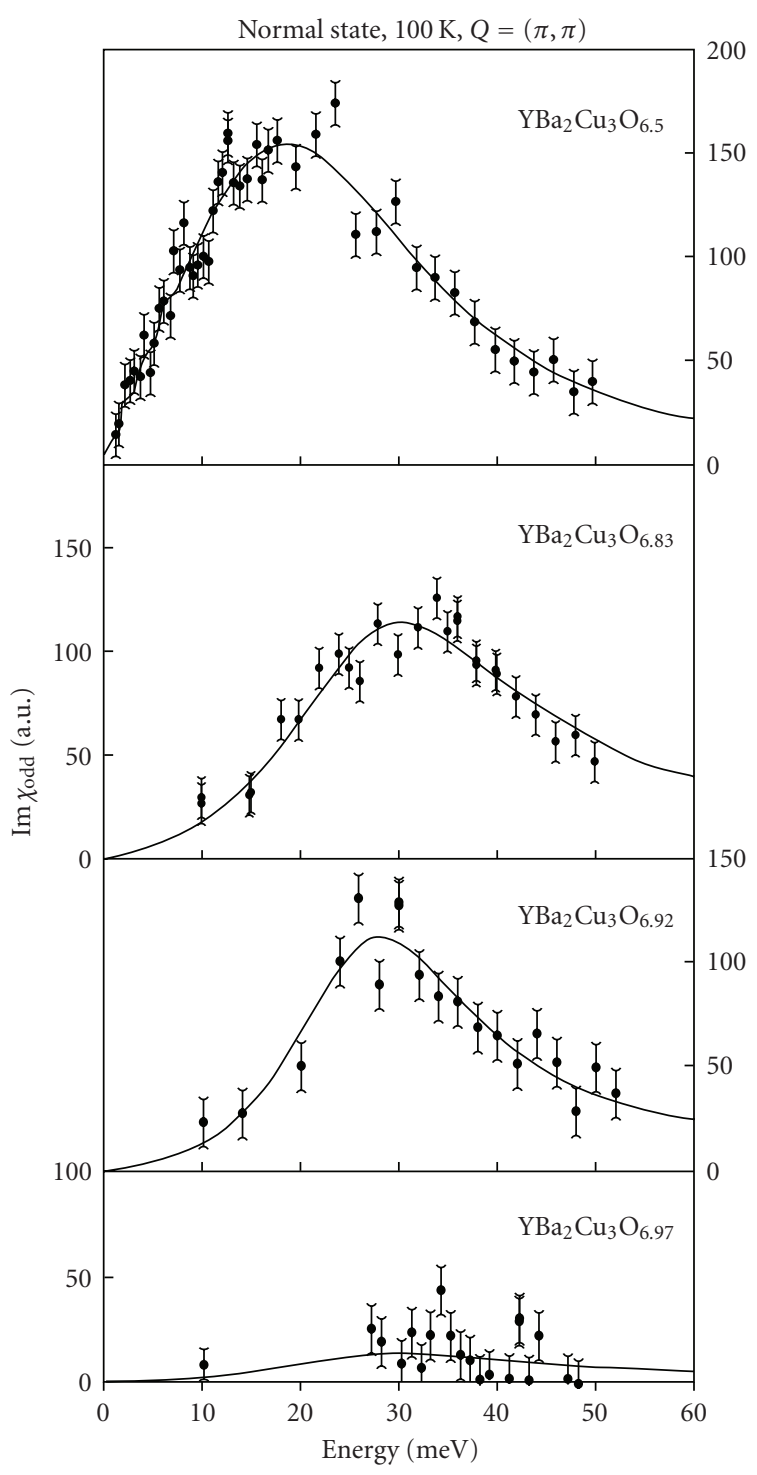

Figure 2: Magnetic spectral function $\operatorname{Im} \chi^{(-)}(\mathbf{k}, \omega)$ in the normal state of $\mathrm{YBa}_{2} \mathrm{Cu}_{3} \mathrm{O}_{6+x}$ at $T=100 \mathrm{~K}$ and at $Q=(\pi, \pi) .100$ counts in the vertical scale correspond to $\chi_{\max }^{(-)} \approx 350 \mu_{B}^{2} / \mathrm{eV}$. The superconducting critical temperature $T_{c}(x)$ by increasing doping $(x)$ from the underdoped system with $x=0.5$ (top) to the optimally doped one with $x=0.97$ (bottom): $T_{c}(x)=45 \mathrm{~K}(x=0.5), 85 \mathrm{~K}$ $(x=0.83), 91 \mathrm{~K}(x=0.92)$, and $92.5 \mathrm{~K}(x=0.97)$. From [30].

of the NMR relaxation rate $T_{1}^{-1}$ which gives $T_{c}^{(\mathrm{NMR})} \approx 100 \mathrm{~K}$ [12-17]. To this point, the NMR measurements (of $T_{1}^{-1}$ ) give that there is an anticorrelation between the decrease of the NMR spectral function $I_{\mathbf{Q}}=\lim _{\omega \rightarrow 0} \operatorname{Im} \chi^{(\mathrm{NMR})}(\mathbf{Q}, \omega) / \omega$ and the increase of $T_{c}$ by increasing doping toward the optimal one-see [6] and references therein. The latter result additionally disfavors the SFI model of pairing [12-17] since the strength of pairing interaction is little affected by SFI. Note that if instead of taking $\operatorname{Im} \chi(\mathbf{Q}, \omega)$ from NMR measurements one takes it from IMNS measurements, as it was done in [82], than for the same value $g_{s f}^{(t h)}$ one obtains much smaller $T_{c}$. For instance, by taking the experimental 
values for $\operatorname{Im} \chi^{(\mathrm{IMNS})}(\mathbf{Q}, \omega)$ in underdoped $\mathrm{YBa}_{2} \mathrm{Cu}_{3} \mathrm{O}_{6.6}$ with $T_{c} \approx 60 \mathrm{~K}$ one obtains $T_{c}^{\text {(IMNS) }}<T_{c}^{(\mathrm{NMR})} / 3$ [82], while $T_{c}^{\text {(IMNS) }} \rightarrow 50 \mathrm{~K}$ for $g_{\text {sf }}^{\text {(th) }} \gg 1$. The situation is even worse if one tries to fit the resistivity with $\operatorname{Im} \chi^{(\mathrm{IMNS})}(\mathbf{Q}, \omega)$ in $\mathrm{YBa}_{2} \mathrm{Cu}_{3} \mathrm{O}_{6.6}$ since this fit gives $T_{c}^{(\mathrm{IMNS})}<7 \mathrm{~K}$. These results point to a deficiency of the SFI phenomenology (at least that based on (10)) to describe pairing in HTSC cuprates.

Having in mind the results in [82], the recent theoretical interpretation in [81] of IMNS experiments [83, 84] and ARPES measurements $[85,86]$ on the underdoped $\mathrm{YBa}_{2} \mathrm{Cu}_{3} \mathrm{O}_{6.6}$ in terms of the SFI phenomenology deserve to be commented. The IMNS experiments $[83,84]$ give evidence for the "hourglass" spin excitation spectrum (in the superconducting state) for the momenta $\mathbf{q}$ at, near and far from $\mathbf{Q}$, which is richer than the common spectrum with magnetic resonance peaks measured at $\mathbf{Q}$. In [81] the self-energy of electrons due to their interaction with spin excitations is calculated by using (10) with $g_{\text {sf }}^{2}=(3 / 2) \widetilde{U}^{2}$ and $\operatorname{Im} \chi(\mathbf{q}, \omega)$ taken from $[83,84]$. However, in order to fit the ARPES self-energy and low-energy kinks (see discussion in Section 1.3.3) the authors of [81] use very large value $\widetilde{U}=1.59 \mathrm{eV}$, that is, much larger than the one used in [82]. Such a large value of $\tilde{U}$ has been obtained earlier within the Monte Carlo simulation of the Hubbard model [87]. In our opinion this value for $\widetilde{U}$ is unrealistically large in the case of strongly correlated systems where spin fluctuations are governed by the effective electron-exchange interaction $J_{\mathrm{Cu}-\mathrm{Cu}} \lesssim 0.15 \mathrm{eV}$ [88]. This implies that $\widetilde{U} \ll 1 \mathrm{eV}$ and $T_{c} \ll$ $60 \mathrm{~K}$. Note that this value for $J_{\mathrm{Cu}-\mathrm{Cu}}(\sim 0.15 \mathrm{eV})$ comes out also from the theory of strongly correlated electrons in the three-band Emery model which gives $J_{\mathrm{Cu}-\mathrm{Cu}} \approx\left[4 t_{p d}^{4} /\left(\Delta_{d p}+\right.\right.$ $\left.\left.U_{p d}\right)^{2},\left(1 / U_{d}\right)+2 /\left(U_{p}+2 \Delta\right)\right]$ - for parameters see Section 2.3. We would like to emphasize here that an additional richness of the spin-fluctuations spectrum (the hourglass instead of the spin resonance) does not change the situation with the smallness of the exchange coupling constant $\tilde{U}$ (and $g_{\text {sf }}$ ).

Concerning the problem related to the rearrangement of the SFI spectral function $\operatorname{Im} \chi(\mathbf{Q}, \omega)$ in $\mathrm{YB}_{2} \mathrm{Cu}_{3} \mathrm{O}_{6+x}[30]$ we would like to stress that despite the fact that the latter results were obtained ten years ago they are not disputed by the new IMNS measurements [31] on high quality samples of the same compound (where much longer counting times were used in order to reduce statistical errors). In fact the results in [30] are confirmed in [31] where the magnetic intensity $I(\mathbf{q}, \omega)(\sim \operatorname{Im} \chi(\mathbf{q}, \omega))$ (for $\mathbf{q}$ at and in the broad range of $\mathbf{Q}$ ) for the optimally doped $\mathrm{YBa}_{2} \mathrm{Cu}_{3} \mathrm{O}_{6.95}$ (with $T_{c}=93 \mathrm{~K}$ ) is at least three times smaller than in the underdoped $\mathrm{YBa}_{2} \mathrm{Cu}_{3} \mathrm{O}_{6.6}$ with $T_{c}=60 \mathrm{~K}$. This result is again very indicative sign of the weakness of SFI since such a huge reconstruction would decrease $T_{c}$ in the optimally doped $\mathrm{YBa}_{2} \mathrm{Cu}_{3} \mathrm{O}_{6.95}$ if analyzed in the framework of the phenomenological SFI theory based on (10). It also implies that due to the suppression of $\operatorname{Im} \chi(\mathbf{q}, \omega)$ by increasing doping toward the optimal one a straightforward extrapolation of the theoretical approach in [81] to the explanation of $T_{c}$ in the optimally doped $\mathrm{YBa}_{2} \mathrm{Cu}_{3} \mathrm{O}_{6.95}$ would require an increase of $\tilde{U}$ to the value even larger than $4 \mathrm{eV}$, which is highly improbable. (b) Ineffectiveness of the Magnetic Resonance Peak. A less direct argument for smallness of the SFI coupling constant, that is, $g_{\text {sf }}^{\exp } \leq 0.2 \mathrm{eV}$ and $g_{\text {sf }}^{\exp } \ll g_{\text {sf }}$, comes from other experiments related to the magnetic resonance peak in the superconducting state, and this will be discussed next. In the superconducting state of optimally doped YBCO and BSCO, $\operatorname{Im} \chi(\mathbf{Q}, \omega)$ is significantly suppressed at low frequencies except near the resonance energy $\omega_{\text {res }} \approx 41 \mathrm{meV}$ where a pronounced narrow peak appears-the magnetic resonance peak. We stress that there is no magnetic resonance peak in some families of HTSC cuprates, for instance, in LSCO, and consequently one can question the importance of the resonance peak in the scattering processes. The experiments tell us that the relative intensity of this peak (compared to the total one) is small, that is, $I_{0} \sim(1-5) \%$-see Figure 3. In underdoped cuprates this peak is present also in the normal state as it is seen in Figure 2.

After the discovery of the resonance peak there were attempts to relate it, first, to the origin of the superconducting condensation energy and, second, to the kink in the energy dispersion or the peak-dimp structure in the ARPES spectral function. In order that the condensation energy is due to the magnetic resonance, it is necessary that the peak intensity $I_{0}$ is small [89]. $I_{0}$ is obtained approximately by equating the condensation energy $E_{\text {con }} \approx N(0) \Delta^{2} / 2$ with the change of the magnetic energy $E_{\text {mag }}$ in the superconducting state, that is, $\delta E_{\mathrm{mag}} \approx 4 I_{0} \cdot E_{\mathrm{mag}}$ :

$$
E_{\mathrm{mag}}=J \iint \frac{d \omega d^{2} k}{(2 \pi)^{3}}\left(1-\cos k_{x}-\cos k_{y}\right) S(\mathbf{k}, \omega),
$$

where $S(\mathbf{k}, \omega)=(1 / \pi)[1+n(\omega)] \operatorname{Im} \chi(\mathbf{k}, \omega)$ is the spin structure factor and $n(\omega)$ is the Bose distribution function. By taking $\Delta \approx 2 T_{c}$ and the realistic value $N(0) \sim 1 /(10 J) \sim$ 1 states/eV $\cdot$ spin, one obtains $I_{0} \sim 10^{-1}\left(T_{c} / J\right)^{2} \sim 10^{-3}$. However, such a small intensity cannot be responsible for the anomalies in ARPES and optical spectra since it gives rise to small coupling constant $\lambda_{\text {sf,res }}$ for the interaction of holes with the resonance peak, that is, $\lambda_{\text {sf,res }} \approx\left(2 I_{0} N(0) g_{\text {sf }}^{2} / \omega_{\text {res }}\right) \ll 1$. Such a small coupling does not affect superconductivity at all. Moreover, by studying the width of the resonance peak one can extract an order of magnitude of the SFI coupling constant $g_{\text {sf }}$. Since the magnetic resonance disappears in the normal state of the optimally doped YBCO, it can be qualitatively understood by assuming that its broadening scales with the resonance energy $\omega_{\text {res }}$, that is, $\gamma_{\text {res }}<\omega_{\text {res }}$, where the line width is given by $\gamma_{\text {res }}=4 \pi\left(N(0) g_{\mathrm{sf}}\right)^{2} \omega_{\text {res }}$ [89]. This condition limits the SFI coupling to $g_{\text {sf }}<0.2 \mathrm{eV}$. We stress that in such a way obtained $g_{\text {sf }}$ is much smaller (at least by factor three) than that assumed in the phenomenological spin-fluctuation theory $[12-17,81]$ where $g_{\text {sf }} \sim 0.6-0.7 \mathrm{eV}$ and $\tilde{U} \approx 1.6 \mathrm{eV}$, but much larger than estimated in [89] (where $g_{s f}<0.02 \mathrm{eV}$ ). The smallness of $g_{s f}$ comes out also from the analysis of the antiferromagnetic state in underdoped metals of LSCO and YBCO [90], where the small (ordered) magnetic moment $\mu\left(<0.1 \mu_{B}\right)$ points to an itinerant antiferromagnetism with small coupling constant $g_{\text {sf }}<0.2 \mathrm{eV}$. The conclusion from this analysis is that in the optimally doped YBCO the sharp magnetic resonance 


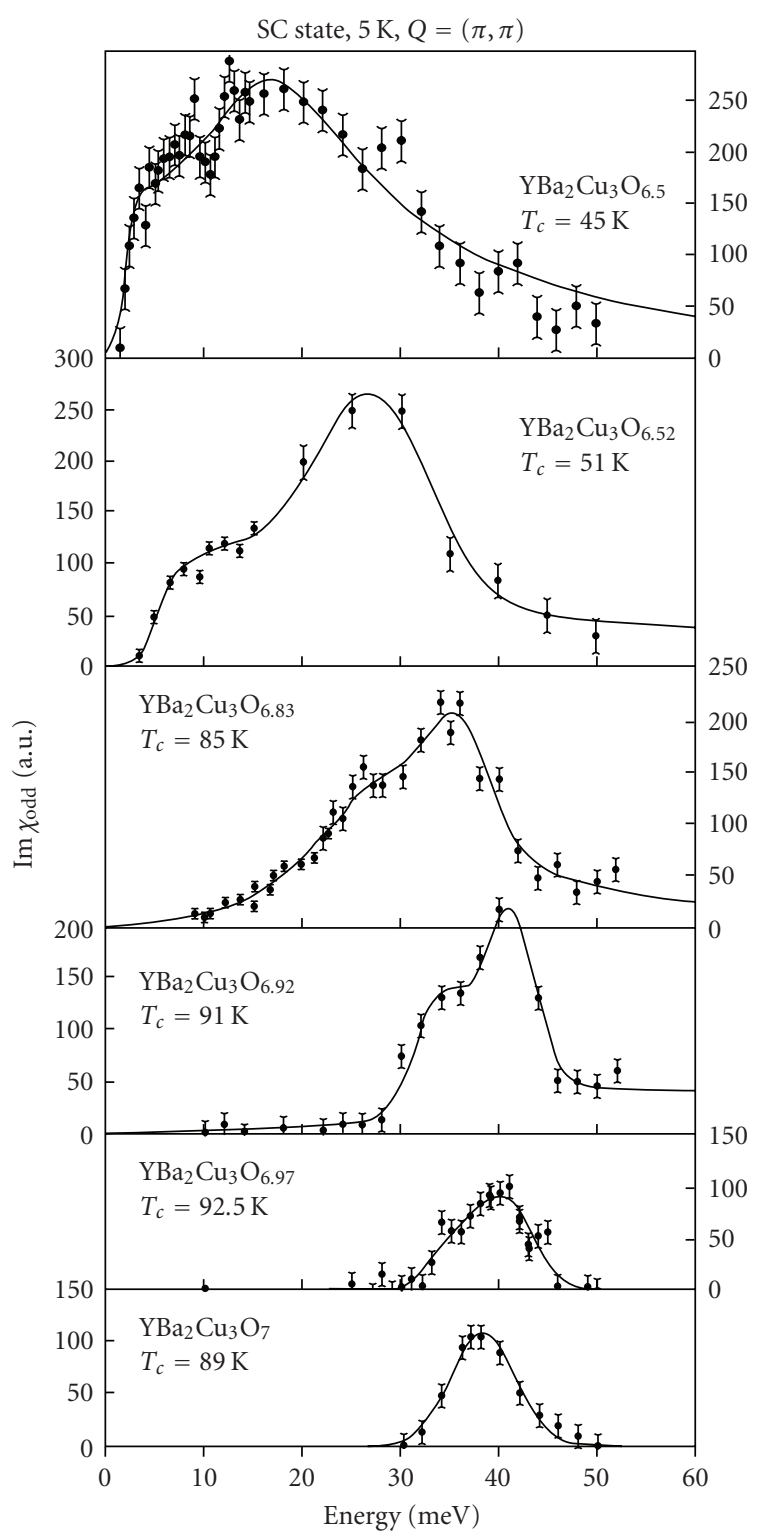

FIGURE 3: Magnetic spectral function $\operatorname{Im} \chi^{(-)}(\mathbf{k}, \omega)$ in the superconducting state of $\mathrm{YBa}_{2} \mathrm{Cu}_{3} \mathrm{O}_{6+x}$ at $T=5 \mathrm{~K}$ and at $Q=(\pi, \pi) .100$ counts in the vertical scale correspond to $\chi_{\max }^{(-)} \approx 350 \mu_{B}^{2} / \mathrm{eV}$. From [30].

is a consequence of the onset of superconductivity and not its cause. There is also one principal reason against the pairing due to the magnetic resonance peak at least in optimally doped cuprates. Since the intensity of the magnetic resonance near $T_{c}$ is vanishingly small, though not affecting pairing at the second-order phase transition at $T_{c}$, then, if it would be solely the origin for superconductivity, the phase transition at $T_{c}$ would be first order, contrary to experiments. Recent ARPES experiments give evidence that the magnetic resonance cannot be related to the kinks in ARPES spectra $[91,92]$ — see the discussion below.

Finally, we would like to point out that the recent magnetic neutron scattering measurements on optimally doped large-volume crystals $\mathrm{Bi}_{2} \mathrm{Sr}_{2} \mathrm{CaCu}_{2} \mathrm{O}_{8+\delta}$ [93], where the absolute value of $\operatorname{Im} \chi(\mathbf{q}, \omega)$ is measured, are questioning also the interpretation of the electronic magnetism in cuprates in terms of the itinerant magnetism. This experiment shows a lack of temperature dependence of the local spin susceptibility $\operatorname{Im} \chi(\omega)=\sum_{q} \operatorname{Im} \chi(\mathbf{q}, \omega)$ across the superconducting transition $T_{c}=91 \mathrm{~K}$, that is, there is only a minimal change in $\operatorname{Im} \chi(\omega)$ between $10 \mathrm{~K}$ and $100 \mathrm{~K}$. Note that if the magnetic excitations were due to itinerant quasiparticles we should have seen dramatic changes of $\operatorname{Im} \chi(\omega)$ as a function of $T$ over the whole energy range. This $T$-independence of $\operatorname{Im} \chi(\omega)$ strongly opposes the theoretical results in [24-27] which assume that the bosonic spectral function is proportional to $\operatorname{Im} \chi(\omega)$ and that the former can be extracted from optic measurements. Namely, the fitting procedure in [24-27] gives that $\operatorname{Im} \chi(\omega)$ is strongly $T$ dependent contrary to the experimental results in [93]—-see more in Section 1.3.2 on optical conductivity.

1.3.2. Optical Conductivity and EPI. Optical spectroscopy gives information on optical conductivity $\sigma(\omega)$ and on twoparticle excitations, from which one can indirectly extract the transport spectral function $\alpha_{\mathrm{tr}}^{2} F(\omega)$. Since this method probes bulk sample (on the skin depth), contrary to ARPES and tunnelling methods which probe tiny regions (10-15 $\AA$ ) near the sample surface, this method is indispensable. However, one should be careful not to overinterpret the experimental results since $\sigma(\omega)$ is not a directly measured quantity but it is derived from the reflectivity $R(\omega)=$ $\left|\left(\sqrt{\varepsilon_{i i}(\omega)}-1\right) /\left(\sqrt{\varepsilon_{i i}(\omega)}+1\right)\right|^{2}$ with the transversal dielectric tensor $\varepsilon_{i i}(\omega)=\varepsilon_{i i, \infty}+\varepsilon_{i i \text {, latt }}+4 \pi i \sigma_{i i}(\omega) / \omega$. Here, $\varepsilon_{i i, \infty}$ is the high-frequency dielectric function, $\varepsilon_{i i}$, latt describes the contribution of the lattice vibrations, and $\sigma_{i i}(\omega)$ describes the optical (dynamical) conductivity of conduction carriers. Since $R(\omega)$ is usually measured in the limited-frequency interval $\omega_{\min }<\omega<\omega_{\max }$, some physical modelling for $R(\omega)$ is needed in order to guess it outside this range-see more in reviews in [3-6]. This was the reason for numerous misinterpretations of optic measurements in cuprates, which will be uncovered below. An illustrative example for this claim is large dispersion in the reported value of $\omega_{\mathrm{pl}}$-from 0.06 to $25 \mathrm{eV}$ - that is, almost three orders of magnitude. However, it turns out that $I R$ measurements of $R(\omega)$ in conjunction with elipsometric measurements of $\varepsilon_{i i}(\omega)$ at high frequencies allow more reliable determination of $\sigma(\omega)$ [94].

(1) Transport and Quasiparticle Relaxation Rates. The widespread misconception in studying the quasiparticle scattering in cuprates was an ad hoc assumption that the transport relaxation rate $\gamma_{\operatorname{tr}}(\omega)$ is equal to the quasiparticle relaxation rate $\gamma(\omega)$, in spite of the well-known fact that the inequality $\gamma_{\operatorname{tr}}(\omega) \neq \gamma(\omega)$ holds in a broad-frequency (energy) region Allen. This (incorrect) assumption was one of the main arguments against the relevance of the EPI scattering mechanism in cuprates. Although we have discussed this problem several times before, we do it again due to the importance of this subject. 
The dynamical conductivity $\sigma(\omega)$ consists of two parts, that is, $\sigma(\omega)=\sigma^{\text {inter }}(\omega)+\sigma^{\text {intra }}(\omega)$ where $\sigma^{\text {inter }}(\omega)$ describes interband transitions which contribute at higher than intraband energies, while $\sigma^{\text {intra }}(\omega)$ is due to intraband transitions which are relevant at low energies $\omega<(1-2) \mathrm{eV}$. (Note that in the $I R$ measurements the frequency is usually given in $\mathrm{cm}^{-1}$, where the following conversion holds: $1 \mathrm{~cm}^{-1}=$ $29.98 \mathrm{GHz}=0.123985 \mathrm{meV}=1.44 \mathrm{~K}$.) The experimental data for $\sigma(\omega)=\sigma_{1}+i \sigma_{2}$ in cuprates are usually processed by the generalized (extended) Drude formula $[32-36,95]$ :

$$
\sigma(\omega)=\frac{\omega_{p}^{2}}{4 \pi} \frac{1}{\gamma_{\mathrm{tr}}(\omega)-i \omega m_{\mathrm{tr}}(\omega) / m_{\infty}} \equiv \frac{i \omega_{p}^{2}}{4 \pi \tilde{\omega}_{\mathrm{tr}}(\omega)},
$$

where $m$ is the mass of the band electrons while the quantity $\widetilde{\omega}_{\text {tr }}(\omega)$ is defined in (19). The expression (12) is a useful representation for systems with single-band electron-boson scattering which is justified in HTSC cuprates. However, this procedure is inadequate for interpreting optical data in multiband systems such as new high-temperature superconductors Fe-based pnictides since even in absence of the inelastic intra- and interband scattering the effective optic relaxation rate may be strongly frequency dependent [96]. (The usefulness of introducing the optic relaxation $\widetilde{\omega}_{\operatorname{tr}}(\omega)$ will be discussed below.) Here, $i=a, b$ enumerates the plane axis; $\omega_{p}, \gamma_{\mathrm{tr}}(\omega, T)$, and $m_{\mathrm{op}}(\omega)$ are the electronic plasma frequency, the transport (optical) scattering rate, and the optical mass, respectively. Very frequently it is analyzed the quantity $\gamma_{\text {tr }}^{*}(\omega, T)$ given by [95]

$$
\gamma_{\mathrm{tr}}^{*}(\omega, T)=\frac{m_{\infty}}{m_{\mathrm{tr}}(\omega)} \gamma_{\mathrm{tr}}(\omega, T)=\frac{\omega \operatorname{Im} \sigma(\omega)}{\operatorname{Re} \sigma(\omega)} .
$$

In the weak coupling limit $\lambda_{e p}<1$, the formula for conductivity given in Appendix A, equations (A.20) and (A.21) can be written in the form of (12) where $\gamma_{\text {tr }}$ reads [3336]

$$
\begin{aligned}
\gamma_{\operatorname{tr}}(\omega, T)=\pi \sum_{l} \int_{0}^{\infty} d \nu \alpha_{\mathrm{tr}, l}^{2} F_{l}(\nu) \\
\times\left[2\left(1+2 n_{B}(\nu)\right)-2 \frac{\nu}{\omega}-\frac{\omega+\nu}{\omega} n_{B}(\omega+\nu)\right. \\
\left.\quad+\frac{\omega-\nu}{\omega} n_{B}(\omega-\nu)\right] .
\end{aligned}
$$

Here $n_{B}(\omega)$ is the Bose distribution function. For completeness we give also the explicit form of the transport mass $m_{\mathrm{tr}}(\omega)$, see $[3-6,32-36]$ :

$$
\frac{m_{\mathrm{tr}}(\omega)}{m_{\infty}}=1+\frac{2}{\omega} \sum_{l} \int_{0}^{\infty} d \nu \alpha_{\mathrm{tr}, l}^{2} F_{l}(\nu) \operatorname{Re} K\left(\frac{\omega}{2 \pi T}, \frac{\nu}{2 \pi T}\right),
$$

with the Kernel $K(x, y)=(i / y)+\{((y-x) / x)[\psi(1-i x+i y)-$ $\psi(1+i y)]\}-\{y \rightarrow-y\}$ where $\psi$ is the di-gamma function. In the presence of impurity scattering one should add $\gamma_{\text {imp,tr }}$ to $\gamma_{\text {tr }}$. It turns out that (14) holds within a few percents also for large $\lambda_{e p}(>1)$. Note that $\alpha_{\mathrm{tr}, l}^{2} F_{l}(\nu) \neq \alpha_{l}^{2} F_{l}(\nu)$ and the index $l$ enumerates all scattering bosons-phonons-spin fluctuations, and so forth. For comparison, the quasiparticle scattering rate $\gamma(\omega, T)$ is given by

$$
\begin{aligned}
\gamma(\omega, T)=2 \pi \int_{0}^{\infty} & d \nu \alpha^{2} F(\nu) \\
& \times\left\{2 n_{B}(\nu)+n_{F}(\nu+\omega)+n_{F}(\nu-\omega)\right\}+\gamma^{\text {imp }},
\end{aligned}
$$

where $n_{F}$ is the Fermi distribution function. For completeness we give also the expression for the quasiparticle effective mass $m^{*}(\omega)$ :

$$
\begin{aligned}
\frac{m^{*}(\omega)}{m}= & 1+\frac{1}{\omega} \sum_{l} \int_{0}^{\infty} d \nu \alpha_{l}^{2} F_{l}(\nu) \\
& \times \operatorname{Re}\left\{\psi\left(\frac{1}{2}+i \frac{\omega+\nu}{2 \pi T}\right)-\psi\left(\frac{1}{2}-i \frac{\omega-\nu}{2 \pi T}\right)\right\} .
\end{aligned}
$$

The term $\gamma^{\text {imp }}$ is due to the impurity scattering. By comparing (14) and (16), it is seen that $\gamma_{\text {tr }}$ and $\gamma$ are different quantities, that is, $\gamma_{\operatorname{tr}} \neq \gamma$, where the former describes the relaxation of Bose particles (excited electron-hole pairs) while the latter one describes the relaxation of Fermi particles. This difference persists also at $T=0 \mathrm{~K}$ where one has (due to simplicity we omit in the following summation over $l$ ) [32]

$$
\begin{gathered}
\gamma_{\mathrm{tr}}(\omega)=\frac{2 \pi}{\omega} \int_{0}^{\omega} d \nu(\omega-\nu) \alpha_{\mathrm{tr}}^{2}(\nu) F(\nu), \\
\gamma(\omega)=2 \pi \int_{0}^{\omega} d \nu \alpha^{2}(\nu) F(\nu) .
\end{gathered}
$$

In the case of EPI with the constant electronic density of states, the above equations give that $\gamma_{e p}(\omega)=$ const for $\omega>$ $\omega_{\mathrm{ph}}^{\max }$ while $\gamma_{e p, \text { tr }}(\omega)$ (as well as $\gamma_{e p \text {,tr }}^{*}$ ) is monotonic growing for $\omega>\omega_{\mathrm{ph}}^{\max }$, where $\omega_{\mathrm{ph}}^{\max }$ is the maximal phonon frequency. So, the growing of $\gamma_{e p \text {,tr }}(\omega)$ (and $\gamma_{e p, \text { tr }}^{*}$ ) for $\omega>\omega_{\mathrm{ph}}^{\max }$ is ubiquitous and natural for the EPI scattering and has nothing to do with some exotic scattering mechanism. This behavior is clearly seen by comparing $\gamma(\omega, T), \gamma_{\mathrm{tr}}(\omega, T)$, and $\gamma_{\mathrm{tr}}^{*}$ which are calculated for the EPI spectral function $\alpha_{e p}^{2}(\omega) F_{\mathrm{ph}}(\omega)$ extracted from tunnelling experiments in YBCO (with $\omega_{\mathrm{ph}}^{\max } \sim 80 \mathrm{meV}$ ) [42-45]—see Figure 4.

The results shown in Figure 4 clearly demonstrate the physical difference between two scattering rates $\gamma_{e p}$ and $\gamma_{e p \text {,tr }}$ (or $\gamma_{\mathrm{tr}}^{*}$ ). It is also seen that $\gamma_{\mathrm{tr}}^{*}(\omega, T)$ is even more linear function of $\omega$ than $\gamma_{\operatorname{tr}}(\omega, T)$. From these calculations one concludes that the quasilinearity of $\gamma_{\operatorname{tr}}(\omega, T)$ (and $\left.\gamma_{\operatorname{tr}}^{*}\right)$ is not in contradiction with the EPI scattering mechanism but it is in fact a natural consequence of EPI. We stress that such behavior of $\gamma_{e p}$ and $\gamma_{e p \text {,tr }}$ (and $\gamma_{e p, \text { tr }}^{*}$ ), shown in Figure 4 , is in fact not exceptional for HTSC cuprates but it is generic for many metallic systems, for instance, 3D metallic oxides, lowtemperature superconductors such as $\mathrm{Al}, \mathrm{Pb}$, and so forthsee more in [3-6] and references therein.

Let us discuss briefly the experimental results for $R(\omega)$ and $\gamma_{\operatorname{tr}}^{*}(\omega, T)$ and compare these with theoretical predictions obtained by using a single-band model with $\alpha_{e p}^{2}(\omega) F_{\mathrm{ph}}(\omega)$ extracted from the tunnelling data with the EPI coupling constant $\lambda_{e p} \gtrsim 2[42-45]$. In the case of YBCO the 


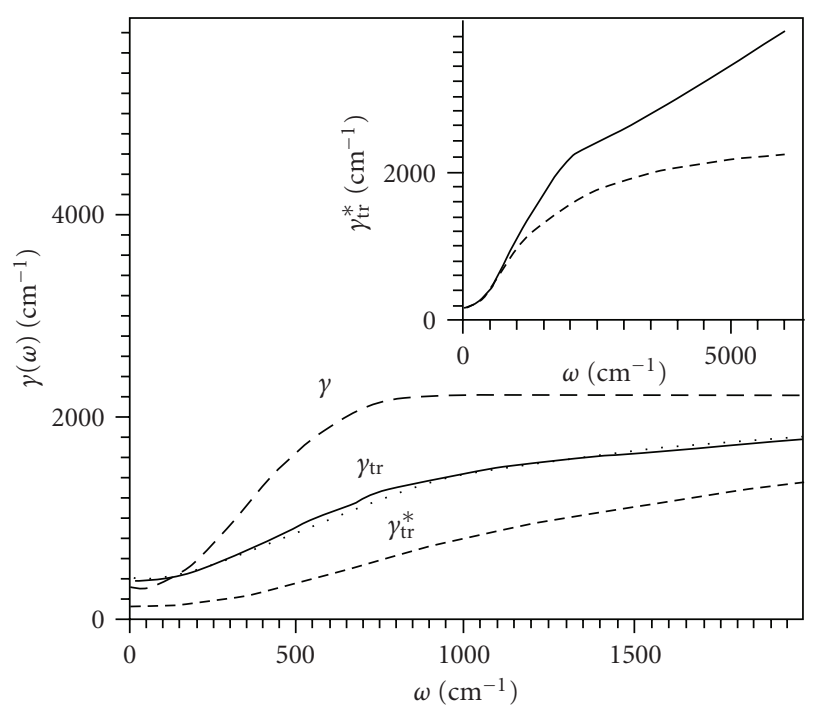

(a)

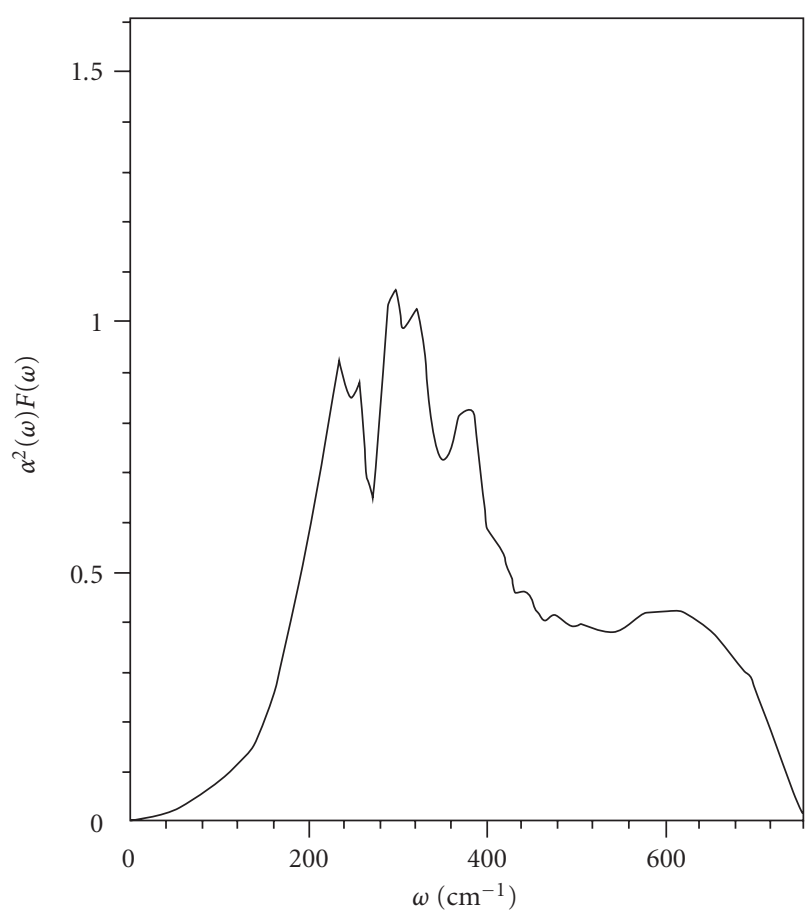

(b)

Figure 4: (a) Scattering rates $\gamma(\omega, T), \gamma_{\text {tr }}(\omega, T)$, and $\gamma_{\text {tr }}^{*}$ from top to bottom-for the Eliashberg function in (b). From [33-35]. (b) Eliashberg spectral function $\alpha_{e p}^{2}(\omega) F_{\mathrm{ph}}(\omega)$ obtained from tunnelling experiments on break junctions [42-45]. Inset shows $\gamma_{\mathrm{tr}}^{*}$ with (full line) and without (dashed line) interband transitions [3-5].

agreement between measured and calculated $R(\omega)$ is very good up to energies $\omega<6000 \mathrm{~cm}^{-1}$, which confirms the importance of EPI in scattering processes. For higher energies, where a mead-infrared peak appears, it is necessary to account for interband transitions [3-5]. In optimally doped $\mathrm{Bi}_{2} \mathrm{Sr}_{2} \mathrm{CaCu}_{2} \mathrm{O}_{6}$ (Bi2212) [97, 98] the experimental results for $\gamma_{\mathrm{tr}}^{*}(\omega, T)$ are explained theoretically by assuming that the EPI spectral function $\alpha_{e p}^{2}(\omega) F(\omega) \sim F_{\mathrm{ph}}(\omega)$, where $F_{\mathrm{ph}}(\omega)$ is the phononic density of states in BSCO, with $\lambda_{e p}=1.9$ and $\gamma_{\mathrm{imp}} \approx 320 \mathrm{~cm}^{-1}$-see Figure 5(a). At the same time the fit of $\gamma_{\mathrm{tr}}^{*}(\omega, T)$ by the marginal Fermi liquid phenomenology fails as it is evident in Figure 5(b).

Now we will comment the so called pronounced linear behavior of $\gamma_{\mathrm{tr}}(\omega, T)$ (and $\gamma_{\mathrm{tr}}^{*}(\omega, T)$ ) which was one of the main arguments for numerous inadequate conclusions regarding the scattering and pairing bosons and EPI. We stress again that the measured quantity is reflectivity $R(\omega)$ and derived ones are $\sigma(\omega), \gamma_{\operatorname{tr}}(\omega, T)$, and $m_{\operatorname{tr}}(\omega)$, which are very sensitive to the value of the dielectric constant $\varepsilon_{\infty}$. This sensitivity is clearly demonstrated in Figure 6 for Bi-2212 where it is seen that $\gamma_{\mathrm{tr}}(\omega, T)$ (and $\gamma_{\mathrm{tr}}^{*}(\omega, T)$ ) for $\varepsilon_{\infty}=1$ is linear up to much higher $\omega$ than in the case $\varepsilon_{\infty}>1$.

However, in some experiments [100-103] the extracted $\gamma_{\operatorname{tr}}(\omega, T)$ (and $\gamma_{\operatorname{tr}}^{*}(\omega, T)$ ) is linear up to very high $\omega \approx$ $1500 \mathrm{~cm}^{-1}$. This means that the ion background and interband transitions (contained in $\varepsilon_{\infty}$ ) are not properly taken into account since too small $\varepsilon_{\infty}(\gtrsim 1)$ is assumed. The recent elipsometric measurements on YBCO [104] give the value $\varepsilon_{\infty} \approx 4-6$, which gives much less spectacular linearity in the relaxation rates $\gamma_{\operatorname{tr}}(\omega, T)$ (and $\gamma_{\operatorname{tr}}^{*}(\omega, T)$ ) than it was the case immediately after the discovery of HTSC cuprates, where much smaller $\varepsilon_{\infty}$ was assumed.

Furthermore, we would like to comment two points related to $\sigma, \gamma_{\text {tr }}$, and $\gamma$. First, the parametrization of $\sigma(\omega)$ with the generalized Drude formula in (12) and its relation to the transport scattering rate $\gamma_{\operatorname{tr}}(\omega, T)$ and the transport mass $m_{\mathrm{tr}}(\omega, T)$ is useful if we deal with electron-boson scattering in a single-band problem. In $[36,96]$ it is shown that $\sigma(\omega)$ of a two-band model with only elastic impurity scattering can be represented by the generalized (extended) Drude formula with $\omega$ and $T$ dependence of effective parameters $\gamma_{\mathrm{tr}}^{\text {eff }}(\omega, T)$, $m_{\mathrm{tr}}^{\text {eff }}(\omega, T)$ despite the fact that the inelastic electron-boson scattering is absent. To this end we stress that the singleband approach is justified for a number of HTSC cuprates such as LSCO, BSCO, and so forth. Second, at the beginning we said that $\gamma_{\operatorname{tr}}(\omega, T)$ and $\gamma(\omega, T)$ are physically different quantities and it holds that $\gamma_{\text {tr }}(\omega, T) \neq \gamma(\omega, T)$. In order to give the physical picture and qualitative explanation for this difference we assume that $\alpha_{\mathrm{tr}}^{2} F(\nu) \approx \alpha^{2} F(\nu)$. In that case the renormalized quasiparticle frequency $\widetilde{\omega}(\omega)=Z(\omega) \omega=$ $\omega-\Sigma(\omega)$ and the transport one $\widetilde{\omega}_{\text {tr }}(\omega)$ - defined in (12) —are related and at $T=0$ they are given by $[32,36]$

$$
\widetilde{\omega}_{\operatorname{tr}}(\omega)=\frac{1}{\omega} \int_{0}^{\omega} d \omega^{\prime} 2 \widetilde{\omega}\left(\omega^{\prime}\right)
$$

(For the definition of $Z(\omega)$ see Appendix A.) It gives the relation between $\gamma_{\text {tr }}(\omega)$ and $\gamma(\omega)$ as well as $m_{\text {tr }}(\omega)$ and $m^{*}(\omega)$, respectively:

$$
\begin{gathered}
\gamma_{\operatorname{tr}}(\omega)=\frac{1}{\omega} \int_{0}^{\omega} d \omega^{\prime} \gamma\left(\omega^{\prime}\right), \\
\omega m_{\mathrm{tr}}(\omega)=\frac{1}{\omega} \int_{0}^{\omega} d \omega^{\prime} 2 \omega^{\prime} m^{*}\left(\omega^{\prime}\right) .
\end{gathered}
$$

The physical meaning of (19) is the following: in optical measurements one photon with the energy $\omega$ is absorbed 


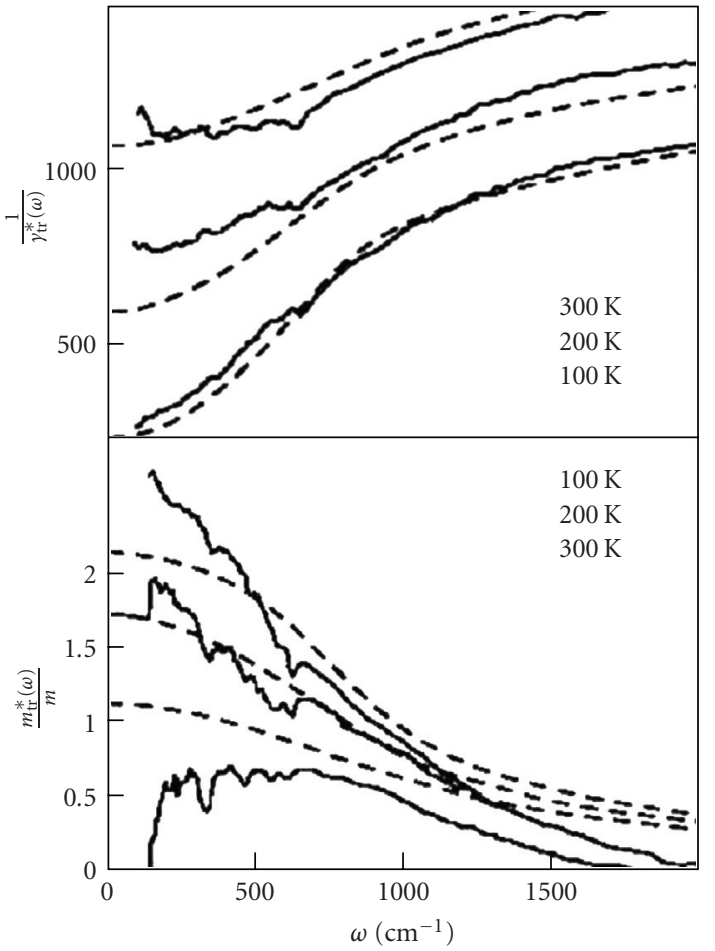

(a)

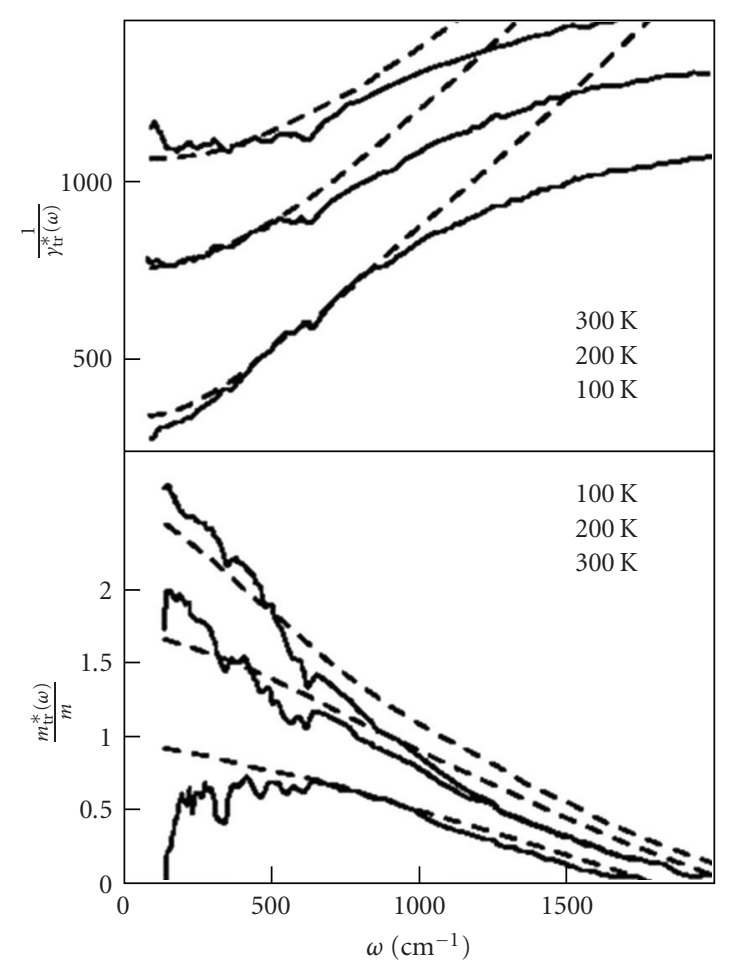

(b)

FIGURE 5: (a) Experimental transport scattering rate $\gamma_{\mathrm{tr}}^{*}$ (solid lines) for BSCO and the theoretical curve by using (A.20) and transport mass $m_{\mathrm{tr}}^{*}$ with $\alpha^{2} F(\omega)$ due to EPI which is described in text (dashed lines). (b) Comparison with the marginal Fermi liquid theory-dashed lines. From $[3-5,99]$.

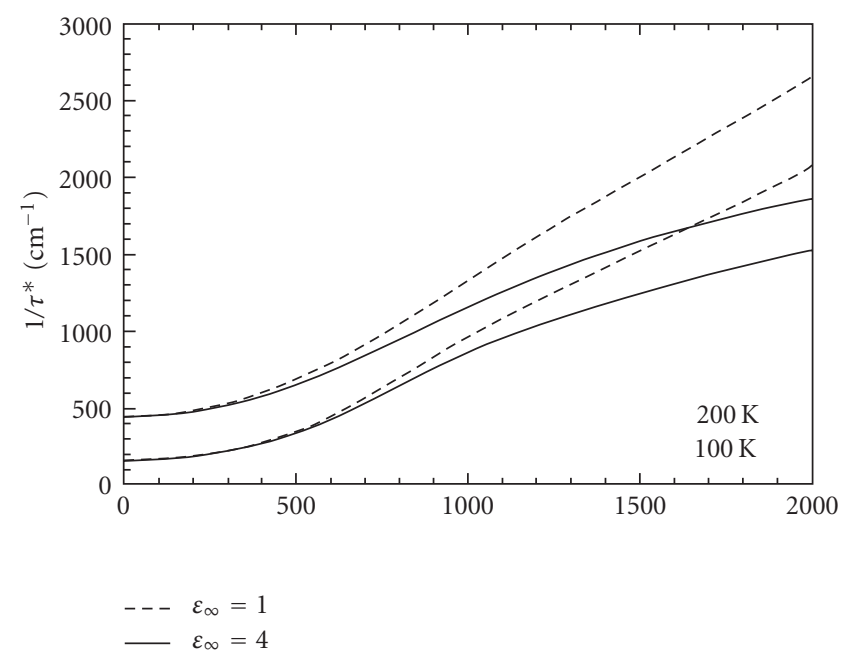

Figure 6: Dependence of $\gamma_{\text {tr }}^{*}(\omega, T)$ on $\varepsilon_{\infty}$ in $\mathrm{Bi}_{2} \mathrm{Sr}_{2} \mathrm{CaCu}_{2} \mathrm{O}_{8}$ for different temperatures: $\varepsilon_{\infty}=4$ (solid lines) and $\varepsilon_{\infty}=1$ (dashed lines). On the horizontal axis is $\omega$ in units $\mathrm{cm}^{-1}$. From [99].

and two excited particles (electron and hole) are created above and below the Fermi surface. If the electron has energy $\omega^{\prime}$ and the hole $\omega-\omega^{\prime}$, then they relax as quasiparticles with the renormalized frequency $\widetilde{\omega}$. Since $\omega^{\prime}$ takes values $0<\omega^{\prime}<\omega$, then the optical relaxation $\widetilde{\omega}_{\operatorname{tr}}(\omega)$ is the energyaveraged $\widetilde{\omega}(\omega)$ according to (19). The factor 2 is due to the two quasiparticles-electron and hole. At finite $T$, the generalization reads $[32,36]$

$$
\tilde{\omega}_{\mathrm{tr}}(\omega)=\frac{1}{\omega} \int_{0}^{\infty} d \omega^{\prime}\left[1-n_{F}\left(\omega^{\prime}\right)-n_{F}\left(\omega-\omega^{\prime}\right)\right] 2 \widetilde{\omega}\left(\omega^{\prime}\right) .
$$

(2) Inversion of the Optical Data and $\alpha_{\mathrm{tr}}^{2}(\omega) F(\omega)$. In principle, the transport spectral function $\alpha_{\mathrm{tr}}^{2}(\omega) F(\omega)$ can be extracted from $\sigma(\omega)$ (or $\left.\gamma_{\operatorname{tr}}(\omega)\right)$ only at $T=0 \mathrm{~K}$, which follows from (18) as

$$
\alpha_{\mathrm{tr}}^{2}(\omega) F(\omega)=\frac{\omega_{p}^{2}}{8 \pi^{2}} \frac{\partial^{2}}{\partial \omega^{2}}\left[\omega \operatorname{Re} \frac{1}{\sigma(\omega)}\right],
$$

or equivalently as $\alpha_{\mathrm{tr}}^{2}(\omega) F(\omega)=(1 / 2 \pi) \partial^{2}\left(\omega \gamma_{\operatorname{tr}}(\omega)\right) / \partial \omega^{2}$. However, real measurements are performed at finite $T$ (at $T>T_{c}$ which is rather high in HTSC cuprates) and the inversion procedure is an ill-posed problem since $\alpha_{\mathrm{tr}}^{2}(\omega) F(\omega)$ is the deconvolution of the inhomogeneous Fredholm integral equation of the first kind with the temperaturedependent Kernel $K_{2}(\omega, v, T)$-see (14). It is known that an ill-posed mathematical problem is very sensitive to input since experimental data contain less information than one needs. This procedure can cause, first, that the fine structure of $\alpha_{\mathrm{tr}}^{2}(\omega) F(\omega)$ get blurred (most peaks are washed out) in the extraction procedures and, second, the extracted $\alpha_{\mathrm{tr}}^{2}(\omega) F(\omega)$ be temperature dependent even when the true 


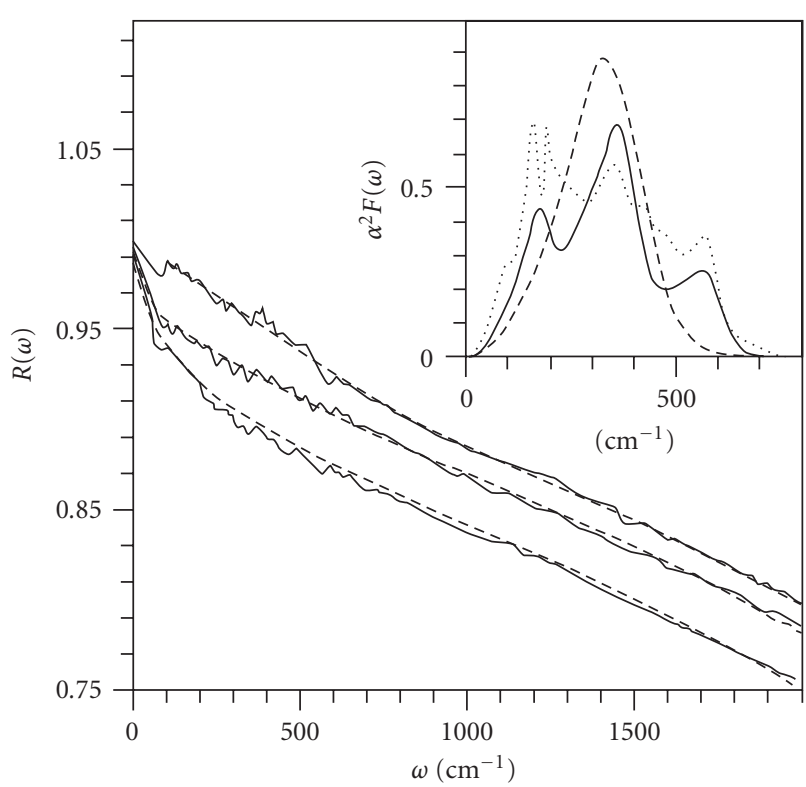

FIgure 7: Experimental (solid lines) and calculated (dashed lines) data of $R(\omega)$ in optimally doped YBCO [105] at $T=100,200,300 \mathrm{~K}$ (from top to bottom). Inset: the two (solid and dashed lines) reconstructed $\alpha_{\mathrm{tr}}^{2}(\omega) F(\omega)$ 's at $T=100 \mathrm{~K}$. The phonon density of states $F(\omega)$-dotted line in the inset. From [33-35].

$\alpha_{\mathrm{tr}}^{2}(\omega) F(\omega)$ is $T$ independent. This artificial $T$ dependence is especially pronounced in HTSC cuprates because $T_{c}(\sim$ $100 \mathrm{~K})$ is very high. In the context of HTSC cuprates, this problem was first studied in [33-36] where this picture is confirmed by the following results: (1) the extracted shape of $\alpha_{\mathrm{tr}}^{2}(\omega) F(\omega)$ in $\mathrm{YBa}_{2} \mathrm{Cu}_{3} \mathrm{O}_{7-x}$ as well as in other cuprates is not unique and it is temperature dependent, that is, at higher $T>T_{c}$ the peak structure is smeared and only a single peak (slightly shifted to higher $\omega$ ) is present. For instance, the experimental data of $R(\omega)$ in YBCO were reproduced by two different spectral functions $\alpha_{\mathrm{tr}}^{2}(\omega) F(\omega)$, one with single peak and the other one with three-peak structure as it is shown in Figure 7, where all spectral functions give almost identical $R(\omega)$. The similar situation is realized in optimally doped BSCO as it is seen in Figure 8 where again different functions $\alpha^{2}(\omega) F(\omega)$ reproduce very well curves for $R(\omega)$ and $\sigma(\omega)$. However, it is important to stress that the obtained width of the extracted $\alpha_{\mathrm{tr}}^{2}(\omega) F(\omega)$ in both compounds coincide with the width of the phonon density of states $F_{\mathrm{ph}}(\omega)$ [33-36, 99]. (2) The upper energy bound for $\alpha_{\mathrm{tr}}^{2}(\omega) F(\omega)$ is extracted in [33-36] and it coincides approximately with the maximal phonon frequency in cuprates $\omega_{\mathrm{ph}}^{\max } \lesssim 80 \mathrm{meV}$ as it is seen in Figures 7 and 8.

These results demonstrate the importance of EPI in cuprates [33-36]. We point out that the width of $\alpha_{\mathrm{tr}}^{2}(\omega) F(\omega)$ which is extracted from the optical measurements [3336] coincides with the width of the quasiparticle spectral function $\alpha^{2}(\omega) F(\omega)$ obtained in tunnelling and ARPES spectra (which we will discuss below), that is, both functions are spread over the energy interval $0<\omega<\omega_{\mathrm{ph}}^{\max }(\lesssim 80 \mathrm{meV})$. Since in cuprates this interval coincides with the width in the phononic density of states $F(\omega)$ and since the maxima of

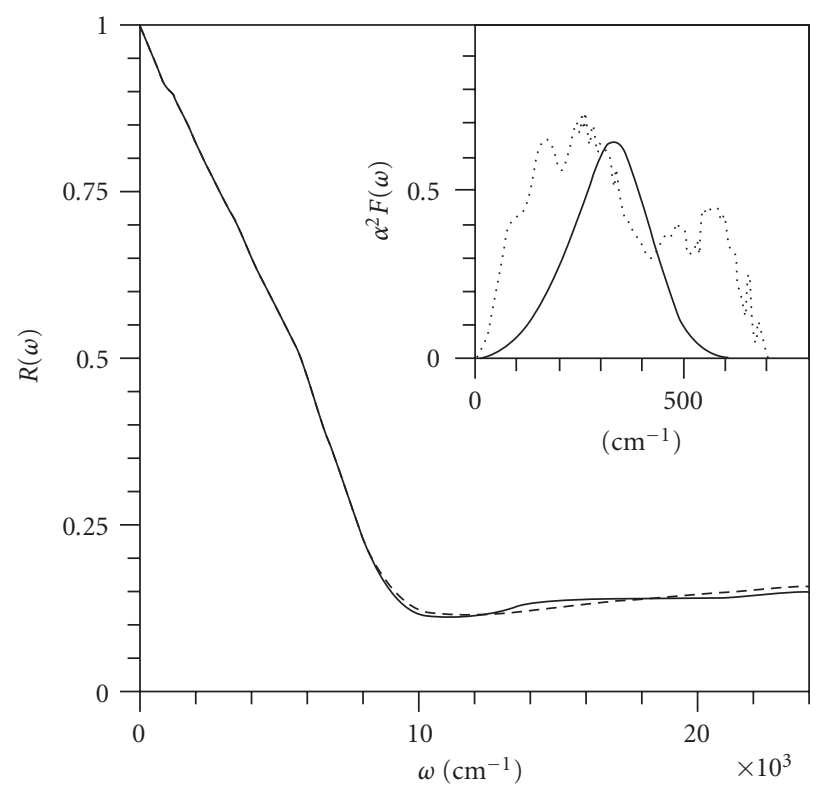

Figure 8: Experimental (solid line) and calculated (dashed line) data of $R(\omega)$ in optimally doped BSCO [106] at $T=100 \mathrm{~K}$. Inset: the reconstructed $\alpha_{\mathrm{tr}}^{2}(\omega) F(\omega)$ - solid line. The phonon density of states $F(\omega)$-dotted line. From [33-35].

$\alpha^{2}(\omega) F(\omega)$ and $F(\omega)$ almost coincide, this is further evidence for the importance of EPI.

To this end, we would like to comment two aspects which appear from time to time in the literature. First, in some reports [24-27] it is assumed that $\alpha_{\mathrm{tr}}^{2}(\omega) F(\omega)$ of cuprates can be extracted also in the superconducting state by using (22). However, (22) holds exclusively in the normal state (at $T=0$ ) since $\sigma(\omega)$ can be described by the generalized (extended) Drude formula in (12) only in the normal state. Such an approach does not hold in the superconducting state since the dynamical conductivity depends not only on the electron-boson scattering but also on coherence factors and on the momentum and energy dependent order parameter $\Delta(\mathbf{k}, \omega)$. Second, if $R(\omega)$ 's (and $\sigma(\omega)$ 's ) in cuprates are due to some other bosonic scattering which is pronounced up to much higher energies $\omega_{c} \gg \omega_{\mathrm{ph}}^{\max }$, this should be seen in the width of the extracted spectral function $\alpha_{\mathrm{tr}}^{2}(\omega) F(\omega)$. In that respect in [25-27] it is assumed that SFI dominates in the quasiparticle scattering and that $\alpha_{\mathrm{tr}}^{2}(\omega) F(\omega) \sim g_{\mathrm{sf}}^{2} \operatorname{Im} \chi(\omega)$ where $\operatorname{Im} \chi(\omega)=\int d^{2} k \chi(\mathbf{k}, \omega)$. This claim is based on reanalyzing of some IR measurements [25-27] and the transport spectral function $\alpha_{\mathrm{tr}}^{2}(\omega) F(\omega)$ is extracted in [25] by using the maximum entropy method in solving the Fredholm equation. However, in order to exclude negative values in the extracted $\alpha_{\mathrm{tr}}^{2}(\omega) F(\omega)$, which is an artefact and due to the chosen method, in [25] it is assumed that $\alpha_{\mathrm{tr}}^{2}(\omega) F(\omega)$ has a rather large tail at large energies-up to $400 \mathrm{meV}$. It turns out that even such an assumption in extracting $\alpha_{\mathrm{tr}}^{2}(\omega) F(\omega)$ does not reproduce the experimental curve $\operatorname{Im} \chi(\omega)$ [107] in some important aspects. First, the relative heights of the two peaks in the extracted spectral function $\alpha_{\mathrm{tr}}^{2}(\omega) F(\omega)$ at lower temperatures are opposite to 
the experimental curve $\operatorname{Im} \chi(\omega)$ [107]—see [25, Figure 1]. Second, the strong temperature dependence of the extracted $\alpha_{\mathrm{tr}}^{2}(\omega) F(\omega)$ found in [25-27] is not an intrinsic property of the spectral function but it is an artefact due to the high sensitivity of the extraction procedure on temperature. As it is already explained before, this is due to the illposed problem of solving the Fredholm integral equation of the first kind with strong T-dependent kernel. Third, the extracted spectral weight $I_{B}(\omega)=\alpha_{\mathrm{tr}}^{2}(\omega) F(\omega)$ in [25] has much smaller values at larger frequencies $(\omega>100 \mathrm{meV})$ than it is the case for the measured $\operatorname{Im} \chi(\omega)$, that is, $\left(I_{B}(\omega>\right.$ $\left.100 \mathrm{meV}) / I_{B}\left(\omega_{\max }\right)\right) \ll \operatorname{Im} \chi(\omega>100 \mathrm{meV}) / \operatorname{Im} \chi\left(\omega_{\max }\right)-$ see [25, Figure 1]. Fourth, the recent magnetic neutron scattering measurements on optimally doped large-volume crystals $\mathrm{Bi}_{2} \mathrm{Sr}_{2} \mathrm{CaCu}_{2} \mathrm{O}_{8+\delta}$ [93] (where the absolute value of $\operatorname{Im} \chi(\mathbf{q}, \omega)$ is measured) are not only questioning the theoretical interpretation of magnetism in HTSC cuprates in terms of itinerant magnetism but also opposing the finding in [25-27]. Namely, this experiment shows that the local spin susceptibility $\operatorname{Im} \chi(\omega)=\sum_{q} \operatorname{Im} \chi(\mathbf{q}, \omega)$ is temperature independent across the superconducting transition $T_{c}=$ $91 \mathrm{~K}$, that is, there is only a minimal change in $\operatorname{Im} \chi(\omega)$ between $10 \mathrm{~K}$ and $100 \mathrm{~K}$. This $T$-independence of $\operatorname{Im} \chi(\omega)$ strongly opposes the (above discussed) results in [2427], where the fit of optic measurements gives strong $T$ dependence of $\operatorname{Im} \chi(\omega)$.

Fifth, the transport coupling constant $\lambda_{\text {tr }}$ extracted in [25] is too large, that is, $\lambda_{\mathrm{tr}}>3$ contrary to the previous findings that $\lambda_{\text {tr }} \lesssim 1.5[33-36,99]$. Since in HTSC one has $\lambda>\lambda_{\text {tr }}$, this would probably give $\lambda \approx 6$, which is not confirmed by other experiments. Sixth, the interpretation of $\alpha_{\mathrm{tr}}^{2}(\omega) F(\omega)$ in LSCO and BSCO solely in terms of $\operatorname{Im} \chi(\omega)$ is in contradiction with the magnetic neutron scattering in the optimally doped and slightly underdoped YBCO [30]. The latter was discussed in Section 1.3.1, where it is shown that $\operatorname{Im} \chi(\mathbf{Q}, \omega)$ is small in the normal state and its magnitude is even below the experimental noise. This means that if the assumption that $\alpha_{\mathrm{tr}}^{2}(\omega) F(\omega) \approx g_{\mathrm{sf}}^{2} \operatorname{Im} \chi(\omega)$ were correct then the contribution to $\operatorname{Im} \chi(\omega)$ from the momenta $0<$ $k \ll Q$ would be dominant, which is detrimental for $d$-wave superconductivity.

Finally, we point out that very similar (to cuprates) properties, of $\sigma(\omega), R(\omega)$ (and $\rho(T)$ and electronic Raman spectra), were observed in 3D isotropic metallic oxides $\mathrm{La}_{0.5} \mathrm{Sr}_{0.5} \mathrm{CoO}_{3}$ and $\mathrm{Ca}_{0.5} \mathrm{Sr}_{0.5} \mathrm{RuO}_{3}$ which are nonsuperconducting [108] and in $\mathrm{Ba}_{1-x} \mathrm{~K}_{x} \mathrm{BiO}_{3}$ which is superconducting below $T_{c} \simeq 30 \mathrm{~K}$ at $x=0.4$. This means that in all of these materials the scattering mechanism might be of similar origin. Since in these compounds there are no signs of antiferromagnetic fluctuations (which are present in cuprates), then the EPI scattering plays important role also in other oxides.

(3) Restricted Optical Sum Rule. The restricted optical sum rule was studied intensively in HTSC cuprates. It shows some peculiarities not present in low-temperature superconductors. It turns out that the restricted spectral weight $W\left(\Omega_{c}, T\right)$ is strongly temperature dependent in the normal and superconducting state, which was interpreted either to be due to EPI $[39,40]$ or to some nonphononic mechanisms [109]. In the following we demonstrate that the temperature dependence of $W\left(\Omega_{c}, T\right)=W(0)-\beta T^{2}$ in the normal state can be explained in a natural way by the $T$ dependence of the EPI transport relaxation rate $\gamma_{\mathrm{tr}}^{e p}(\omega, T)[39,40]$. Since the problem of the restricted sum rule has attracted much interest, it will be considered here in some details. In fact, there are two kinds of sum rules related to $\sigma(\omega)$. The first one is the total sum rule which in the normal state reads

$$
\int_{0}^{\infty} d \omega \sigma_{1}^{N}(\omega)=\frac{\omega_{\mathrm{pl}}^{2}}{8}=\frac{\pi n e^{2}}{2 m},
$$

while in the superconducting state it is given by the TinkhamFerrell-Glover (TFG) sum rule

$$
\int_{0}^{\infty} d \omega \sigma_{1}^{S}(\omega)=\frac{c^{2}}{8 \lambda_{L}^{2}}+\int_{+0}^{\infty} d \omega \sigma_{1}^{S}(\omega)=\frac{\omega_{\mathrm{pl}}^{2}}{8} .
$$

Here, $n$ is the total electron density, $e$ is the electron charge, $m$ is the bare electron mass, and $\lambda_{L}$ is the London penetration depth. The first (singular) term $c^{2} / 8 \lambda_{L}^{2}$ in (24) is due to the superconducting condensate which contributes $\sigma_{1, \text { cond }}^{S}(\omega)=\left(c^{2} / 4 \lambda_{L}^{2}\right) \delta(\omega)$. The total sum rule represents the fundamental property of matter-the conservation of the electron number. In order to calculate it one should use the total Hamiltonian $\hat{H}_{\text {tot }}=\widehat{T}_{e}+\widehat{H}_{\text {int }}$ where all electrons, electronic bands, and their interactions $\hat{H}_{\text {int }}$ (Coulomb, EPI, with impurities, etc.) are accounted for. Here, $T_{e}$ is the kinetic energy of bare electrons:

$$
\widehat{T}_{e}=\sum_{\sigma} \int d^{3} x \hat{\psi}_{\sigma}^{\dagger}(x) \frac{\hat{\mathbf{p}}^{2}}{2 m} \hat{\psi}_{\sigma}(x)=\sum_{\mathbf{p}, \sigma} \frac{\mathbf{p}^{2}}{2 m_{e}} \hat{c}_{\mathbf{p} \sigma}^{\dagger} \hat{c}_{\mathbf{p} \sigma} .
$$

The partial sum rule is related to the energetics solely in the conduction (valence) band which is described by the Hamiltonian of the conduction (valence) band electrons:

$$
\hat{H}_{v}=\sum_{\mathbf{p}, \sigma} \xi_{\mathbf{p}} \hat{c}_{v, \mathbf{p} \sigma}^{\dagger} \hat{c}_{v, \mathbf{p} \sigma}+\hat{V}_{v, c}
$$

$\hat{H}_{v}$ contains the band energy with the dispersion $\epsilon_{\mathbf{p}}\left(\xi_{\mathbf{p}}=\right.$ $\left.\epsilon_{\mathbf{p}}-\mu\right)$ and the effective Coulomb interaction of the valence electrons $\hat{V}_{v, c}$. In this case the partial sum rule in the normal state reads [110] (for a general form of $\epsilon_{\mathbf{p}}$ )

$$
\int_{0}^{\infty} d \omega \sigma_{1, v}^{N}(\omega)=\frac{\pi e^{2}}{2 V} \sum_{\mathbf{p}} \frac{\left\langle\hat{n}_{v, \mathbf{p}}\right\rangle_{H_{v}}}{m_{\mathbf{p}}},
$$

where the number operator $\hat{n}_{v, \mathbf{p}}=\sum_{\sigma} \hat{c}_{\mathbf{p} \sigma}^{\dagger} \hat{c}_{\mathbf{p} \sigma} ; 1 / m_{\mathbf{p}}=$ $\partial^{2} \epsilon_{\mathrm{p}} / \partial p_{x}^{2}$ is the momentum-dependent reciprocal mass and $V$ is volume. In practice, the optic measurements are performed up to finite frequency and the integration over $\omega$ goes up to some cutoff frequency $\Omega_{c}$ (of the order of the band plasma frequency). In this case the restricted sum rule has the form

$$
\begin{aligned}
W\left(\Omega_{c}, T\right) & =\int_{0}^{\Omega_{c}} d \omega \sigma_{1, v}^{N}(\omega) \\
& =\frac{\pi}{2}\left[K^{d}+\Pi(0)\right]-\int_{0}^{\Omega_{c}} d \omega \frac{\operatorname{Im} \Pi(\omega)}{\omega},
\end{aligned}
$$


where $K^{d}$ is the diamagnetic Kernel given by (30) below and $\Pi(\omega)$ is the paramagnetic (current-current) response function. In the perturbation theory without vertex correction $\Pi\left(i \omega_{n}\right)$ (at the Matsubara frequency $\omega_{n}=\pi T(2 n+1)$ ) is given by $[39,40]$

$$
\Pi(i \omega)=2 \sum_{\mathbf{p}}\left(\frac{\partial \epsilon_{\mathbf{p}}}{\partial \mathbf{p}}\right)^{2} \sum_{\omega_{m}} G\left(\mathbf{p}, i \omega_{n m}^{+}\right) G\left(\mathbf{p}, i \omega_{m}\right),
$$

where $\omega_{n m}^{+}=\omega_{n}+\omega_{m}$ and $G\left(\mathbf{p}, i \omega_{n}\right)=\left(i \omega_{n}-\xi_{\mathbf{p}}-\right.$ $\left.\Sigma\left(\mathbf{p}, i \omega_{n}\right)\right)^{-1}$ is the electron Green's function. In the case when the interband gap $E_{g}$ is the largest scale in the problem, that is, when $W_{b}<\Omega_{c}<E_{g}$, in this region one has approximately $\operatorname{Im} \Pi(\omega) \approx 0$ and the limit $\Omega_{c} \rightarrow \infty$ in (28) is justified. In that case one has $\Pi(0) \approx \int_{0}^{\Omega_{c}}(\operatorname{Im} \Pi(\omega) / \omega) d \omega$ which gives the approximate formula for $W\left(\Omega_{c}, T\right)$ :

$$
\begin{aligned}
W\left(\Omega_{c}, T\right) & =\int_{0}^{\Omega_{c}} d \omega \sigma_{1, v}^{N}(\omega) \approx \frac{\pi}{2} K^{d} \\
& =e^{2} \pi \sum_{\mathbf{p}} \frac{\partial^{2} \epsilon_{\mathbf{p}}}{\partial \mathbf{p}^{2}} n_{\mathbf{p}},
\end{aligned}
$$

where $n_{\mathbf{p}}=\left\langle\hat{n}_{v, \mathbf{p}}\right\rangle$ is the quasiparticle distribution function in the interacting system. Note that $W\left(\Omega_{c}, T\right)$ is cutoff dependent while $K^{d}$ in (30) does not depend on $\Omega_{c}$. So, one should be careful not to overinterpret the experimental results in cuprates by this formula. In that respect the best way is to calculate $W\left(\Omega_{c}, T\right)$ by using the exact result in (28) which apparently depends on $\Omega_{c}$. However, (30) is useful for appropriately chosen $\Omega_{c}$, since it allows us to obtain semiquantitative results. In most papers related to the restricted sum rule in HTSC cuprates it was assumed, due to simplicity, the tight-binding model with nearest neighbors (n.n.) with the energy $\epsilon_{\mathrm{p}}=-2 t\left(\cos p_{x} a+\cos p_{y} a\right)$ which gives $1 / m_{\mathrm{p}}=-2 t a^{2} \cos p_{x} a$. It is straightforward to show that in this case (30) is reduced to a simpler one:

$$
\begin{aligned}
W\left(\Omega_{c}, T\right) & =\int_{0}^{\Omega_{c}} d \omega \sigma_{1, v}^{N}(\omega) \\
& \approx \frac{\pi e^{2} a^{2}}{2 V}\left\langle-T_{v}\right\rangle,
\end{aligned}
$$

where $\left\langle T_{v}\right\rangle_{H_{v}}=\sum_{\mathbf{p}} \epsilon_{\mathbf{p}}\left\langle n_{v}\right\rangle_{H_{v}}$ is the average kinetic energy of the band electrons, $a$ is the $\mathrm{Cu}-\mathrm{Cu}$ lattice distance, and $V$ is the volume of the system. In this approximation $W\left(\Omega_{c}, T\right)$ is a direct measure of the average band (kinetic) energy. In the superconducting state the partial band sum rule reads

$$
\begin{aligned}
W_{s}\left(\Omega_{c}, T\right) & =\frac{c^{2}}{8 \lambda_{L}^{2}}+\int_{+0}^{\Omega_{c}} d \omega \sigma_{1, v}^{S}(\omega) \\
& =\frac{\pi e^{2} a^{2}}{2 V}\left\langle-T_{v}\right\rangle_{s} .
\end{aligned}
$$

In order to introduce the reader to (the complexity of) the problem of the $T$ dependence of $W\left(\Omega_{c}, T\right)$, let us consider the electronic system in the normal state and in absence of the quasiparticle interaction. In that case one has $n_{\mathbf{p}}=f_{\mathbf{p}}\left(f_{\mathbf{p}}\right.$

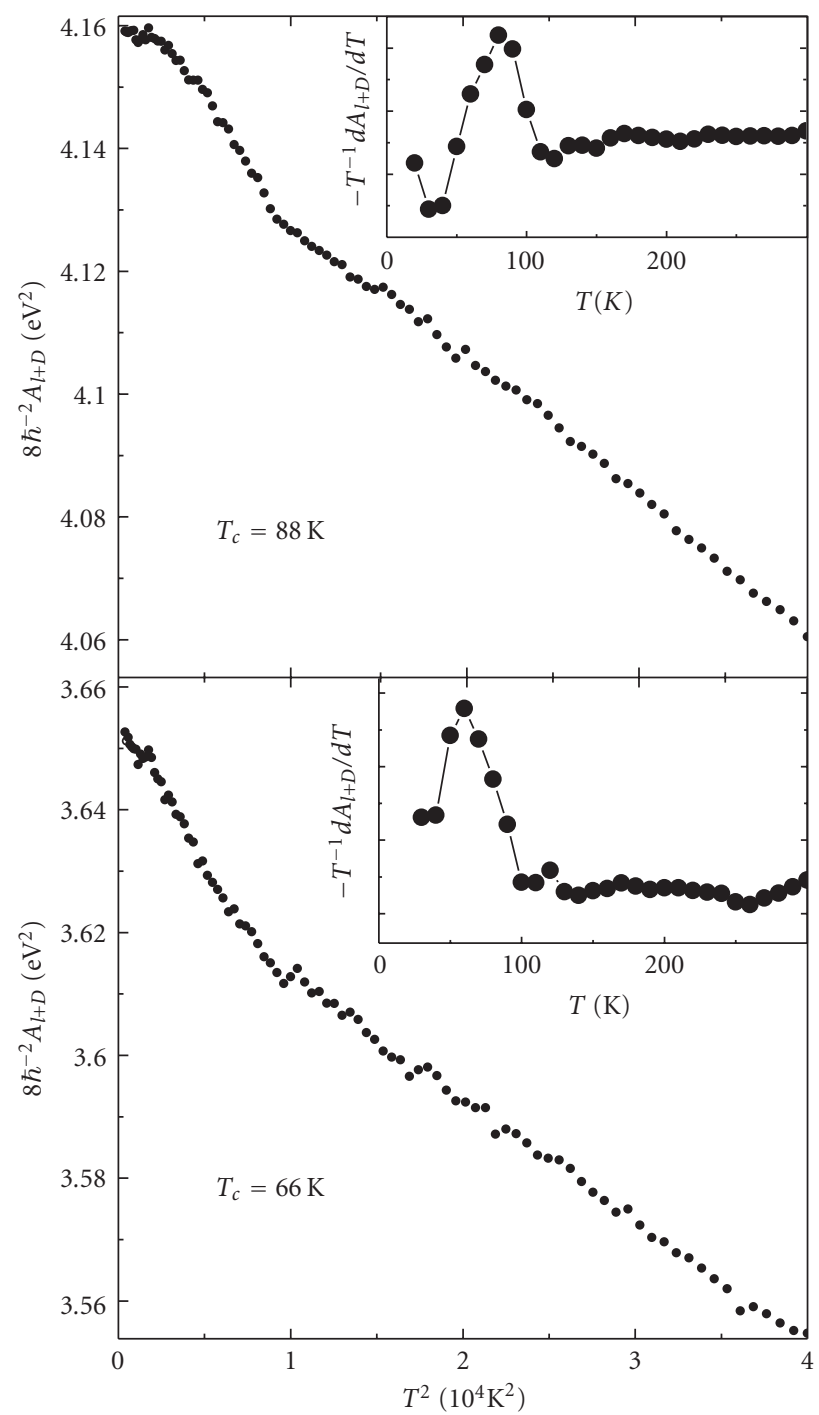

FIGURE 9: Measured spectral weight $W_{s}\left(\Omega_{c}, T\right)\left(\sim A_{l+D}\right.$ in figures $)$ for $\Omega_{c} \approx 1.25 \mathrm{eV}$ in two underdoped Bi2212 (with $T_{c}=88 \mathrm{~K}$ and $\left.T_{c}=66 \mathrm{~K}\right)$. From [111].

is the Fermi distribution function) and $W_{n}\left(\Omega_{c}, T\right)$ increases with the decrease of the temperature, that is, $W_{n}\left(\Omega_{c}, T\right)=$ $W_{n}(0)-\beta_{b} T^{2}$ where $\beta_{b} \sim 1 / W_{b}$. To this end, let us mention in advance that the experimental value $\beta_{\exp }$ is much larger than $\beta_{b}$, that is, $\beta_{\exp } \gg \beta_{b}$, thus telling us that the simple Sommerfeld-like smearing of $f_{\mathrm{p}}$ by the temperature effects cannot explain quantitatively the $T$ dependence of $W\left(\Omega_{c}, T\right)$. We stress that the smearing of $f_{\mathbf{p}}$ by temperature lowers the spectral weight compared to that at $T=0 \mathrm{~K}$, that is, $W_{n}\left(\Omega_{c}, T\right)<W_{n}\left(\Omega_{c}, 0\right)$. In that respect it is not surprising that there is a lowering of $W_{s}\left(\Omega_{c}, T\right)$ in the BCS superconducting state, $W_{s}^{\mathrm{BCS}}\left(\Omega_{c}, T \ll T_{c}\right)<W_{n}\left(\Omega_{c}, T \ll\right.$ $T_{c}$ ) since at low temperatures $f_{\mathbf{p}}$ is smeared mainly due to the superconducting gap, that is, $f_{\mathbf{p}}=\left[1-\left(\xi_{\mathbf{p}} / E_{\mathbf{p}}\right) \operatorname{th}\left(E_{\mathbf{p}} / 2 T\right)\right] / 2$, $E_{\mathbf{p}}=\sqrt{\xi_{\mathbf{p}}^{2}+\Delta^{2}}, \xi_{\mathbf{p}}=\epsilon_{\mathbf{p}}-\mu$. The maximal decrease of $W_{s}\left(\Omega_{c}, T\right)$ is at $T=0$. 


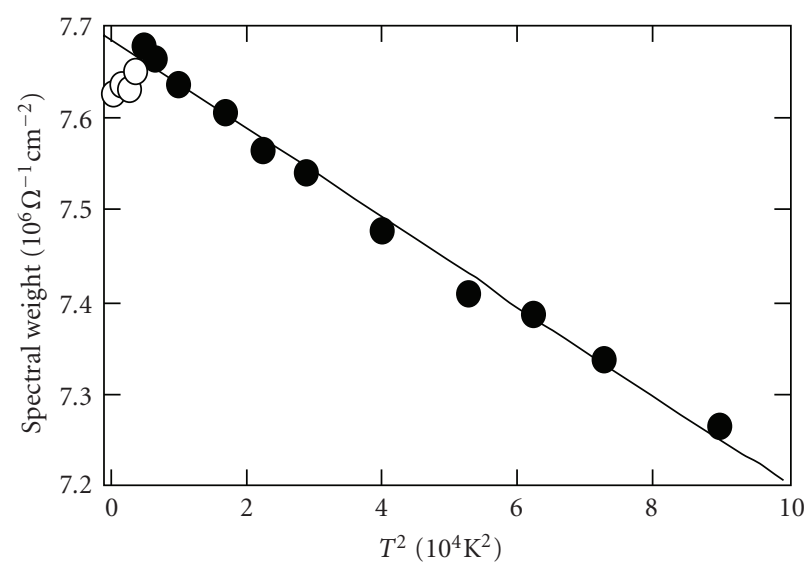

(a)

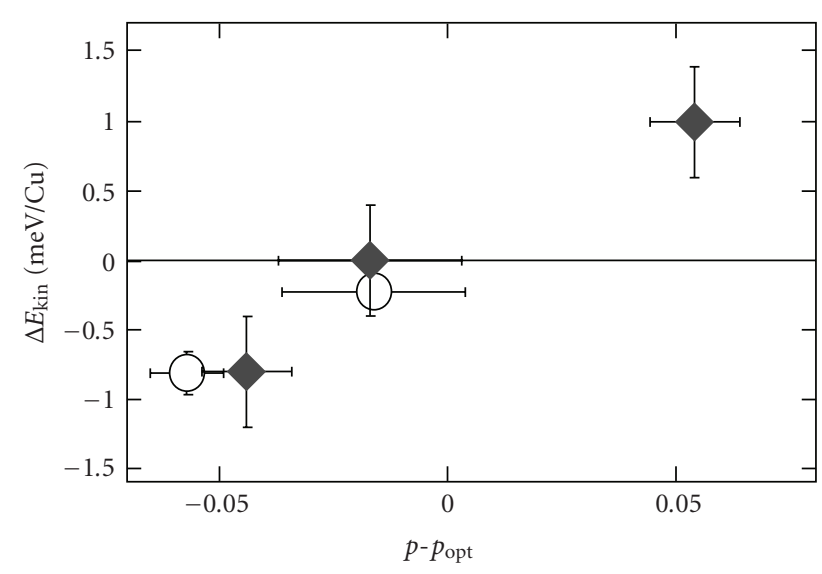

(b)

Figure 10: (a) Spectral weight $W_{n}\left(\Omega_{c}, T\right)$ of the overdoped Bi2212 for $\Omega_{c}=1 \mathrm{eV}$. Closed symbols-normal state. Open symbolssuperconducting state. (b) Change of the kinetic energy $\Delta E_{\text {kin }}=$ $E_{\mathrm{kin}, S}-E_{\mathrm{kin}, N}$ in meV per $\mathrm{Cu}$ site versus the charge $p$ per $\mathrm{Cu}$ with respect to the optimal value $p_{\text {opt }}$. From [112].

Let us enumerate and discuss the main experimental results for $W\left(\Omega_{c}, T\right)$ in HTSC cuprates. (1) In the normal state $\left(T>T_{c}\right)$ of most cuprates, one has $W_{n}\left(\Omega_{c}, T\right)=W_{n}(0)-$ $\beta_{\text {ex }} T^{2}$ with $\beta_{\exp } \gg \beta_{b}$, that is, $W_{n}\left(\Omega_{c}, T\right)$ is increasing by decreasing $T$, even at $T$ below $T^{*}$ - the temperature for the opening of the pseudogap. The increase of $W_{n}\left(\Omega_{c}, T\right)$ from the room temperature down to $T_{c}$ is no more than $5 \%$. (2) In the superconducting state $\left(T<T_{c}\right)$ of some underdoped and optimally doped Bi-2212 compounds [111, 113, 114] (and underdoped Bi-2212 films [115]) there is an effective increase of $W_{s}\left(\Omega_{c}, T\right)$ with respect to that in the normal state, that is, $W_{s}\left(\Omega_{c}, T\right)>W_{n}\left(\Omega_{c}, T\right)$ for $T<T_{c}$. This is a non-BCS behavior which is shown in Figure 9. Note that in the tight binding model the effective band (kinetic) energy $\left\langle T_{v}\right\rangle$ is negative $\left(\left\langle T_{v}\right\rangle<0\right)$ and in the standard BCS case (32) gives that $W_{s}\left(T<T_{c}\right)$ decreases due to the increase of $\left\langle T_{v}\right\rangle$. Therefore the experimental increase of $W_{s}(T<$ $T_{c}$ ) by decreasing $T$ is called the non-BCS behavior. The latter means a lowering of the kinetic energy $\left\langle T_{v}\right\rangle$ which is frequently interpreted to be due either to strong correlations or to a Bose-Einstein condensation (BEC) of the preformed tightly bound Cooper pair-bosons, for instance, bipolarons [116]. It is known that in the latter case the kinetic energy of bosons is decreased below the BEC critical temperature $T_{c}$. In [117] it is speculated that the latter case might be realized in underdoped cuprates.

However, in some optimally doped and in most overdoped cuprates, there is a decrease of $W_{s}\left(\Omega_{c}, T\right)$ at $T<T_{c}$ $\left(W_{s}\left(\Omega_{c}, T\right)<W_{n}\left(\Omega_{c}, T\right)\right)$ which is the BCS-like behavior [112] as it is seen in Figure 10.

We stress that the non-BCS behavior of $W_{s}\left(\Omega_{c}, T\right)$ for underdoped (and in some optimally doped) systems was obtained by assuming that $\Omega_{c} \approx(1-1.2) \mathrm{eV}$. However, in [104] these results were questioned by claiming that the conventional BCS-like behavior was observed $\left(W_{s}\left(\Omega_{c}, T\right)<\right.$ $\left.W_{n}\left(\Omega_{c}, T\right)\right)$ in the optimally doped YBCO and slightly underdoped Bi-2212 by using larger cutoff energy $\Omega_{c}=$ $1.5 \mathrm{eV}$. This discussion demonstrates how risky is to make definite conclusions on some fundamental physics based on the parameter- (such as the cutoff energy $\Omega_{c}$ ) dependent analysis. Although the results obtained in [104] look very trustfully, it is fair to say that the issue of the reduced spectral weight in the superconducting state of the underdoped cuprates is still unsettled and under dispute. In overdoped Bi-2212 films, the BCS-like behavior $W_{s}\left(\Omega_{c}, T\right)<W_{n}\left(\Omega_{c}, T\right)$ was observed, while in LSCO it was found that $W_{s}\left(\Omega_{c}, T\right) \approx$ const, that is, $W_{s}\left(\Omega_{c}, T<T_{c}\right) \approx W_{n}\left(\Omega_{c}, T_{c}\right)$.

The first question is the following. How to explain the strong temperature dependence of $W\left(\Omega_{c}, T\right)$ in the normal state? In $[39,40] W(T)$ is explained solely in the framework of the EPI physics where the EPI relaxation $\gamma_{e p}(T)$ plays the main role in the $T$ dependence of $W\left(\Omega_{c}, T\right)$. The main theoretical results of $[39,40]$ are the following: the calculations of $W(T)$ based on the exact (30) give that for $\Omega_{c} \gg \Omega_{D}$ (the Debye energy) the difference in spectral weights of the normal and superconducting states is small, that is, $W_{n}\left(\Omega_{c}, T\right) \approx W_{s}\left(\Omega_{c}, T\right)$ since $W_{n}\left(\Omega_{c}, T\right)-$ $W_{s}\left(\Omega_{c}, T\right) \sim \Delta^{2} / \Omega_{c}^{2}$. (2) In the case of large $\Omega_{c}$ the calculations based on (30) give

$$
W\left(\Omega_{c}, T\right) \approx \frac{\omega_{\mathrm{pl}}^{2}}{8}\left[1-\frac{\gamma(T)}{W_{b}}-\frac{\pi^{2}}{2} \frac{T^{2}}{W_{b}^{2}}\right] .
$$

In the case when EPI dominates one has $\gamma=\gamma_{e p}(T)+\gamma_{\text {imp }}$ where $\gamma_{e p}(T)=\int_{0}^{\infty} d z \alpha^{2}(z) F(z) \operatorname{coth}(z / 2 T)$. It turns out that for $\alpha^{2}(\omega) F(\omega)$, shown in Figure 4, one obtains (i) $\gamma_{e p}(T) \sim$ $T^{2}$ in the temperature interval $100 \mathrm{~K}<T<200 \mathrm{~K}$ as it is seen in Figure 11 for the $T$ dependence of $W\left(\Omega_{c}, T\right)[39,40]$; (ii) the second term in (33) is much larger than the last one (the Sommerfeld-like term). For the EPI coupling constant $\lambda_{e p, \text { tr }}=1.5$ one obtains rather good agreement between the theory in $[39,40]$ and experiments in $[104,111,113,114]$. At lower temperatures, $\gamma_{e p}(T)$ deviates from the $T^{2}$ behavior and this deviation depends on the structure of the spectrum in $\alpha^{2}(\omega) F(\omega)$. It is seen in Figure 11 that, for a softer Einstein spectrum (with $\Omega_{E}=200 \mathrm{~K}$ ), $W\left(\Omega_{c}, T\right)$ lies above the curve with the $T^{2}$ asymptotic behavior, while the curve with a harder phononic spectrum (with $\Omega_{E}=400 \mathrm{~K}$ ) lies below it. This result means that different behavior of $W\left(\Omega_{c}, T\right)$ in 


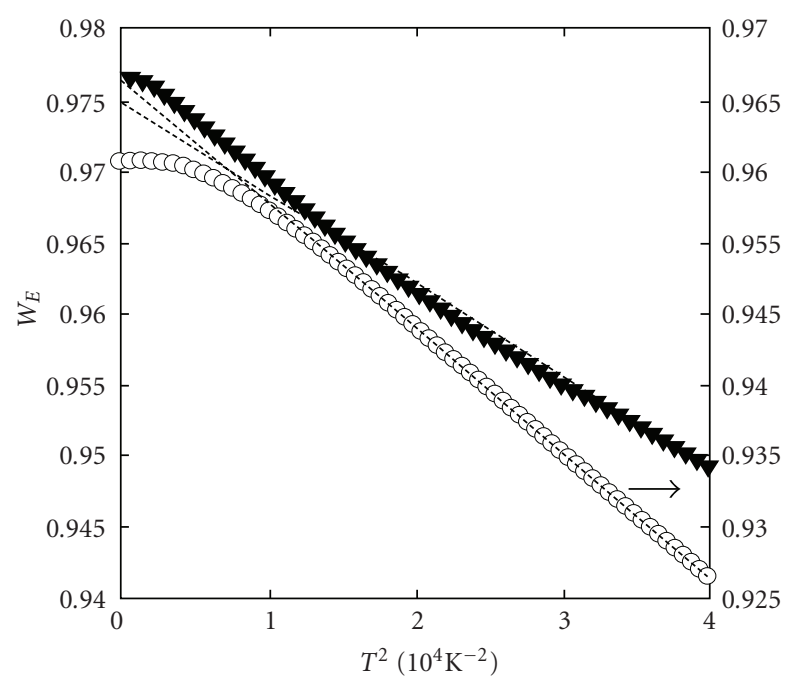

Figure 11: Spectral weight $W\left(\Omega_{c}, T\right)$ in the normal state for Einstein phonons with $\Omega_{E}=200 \mathrm{~K}$ (full triangles) and $\Omega_{E}=400 \mathrm{~K}$ (open circles, left axis). Dashed lines show $T^{2}$ asymptotic. From [40].

the superconducting state of cuprates for different doping might be simply related to different contributions of lowand high-frequency phonons. We stress that such a behavior of $W\left(\Omega_{c}, T\right)$ was observed in experiments in $[104,111,113$, $114]$.

To summarize, the above analysis demonstrates that the theory based on EPI is able to explain in a satisfactory way the temperature behavior of $W\left(\Omega_{c}, T\right)$ above and below $T_{c}$ in systems at and near the optimal doping.

(4) $\alpha^{2}(\omega) F(\omega)$ and the In-Plane Resistivity $\rho_{a b}(T)$. The temperature dependence of the in-plane resistivity $\rho_{a b}(T)$ in cuprates is a direct consequence of the quasi-2D motion of quasiparticles and of the inelastic scattering which they experience. At present, there is no consensus on the origin of the linear temperature dependence of the in-plane resistivity $\rho_{a b}(T)$ in the normal state. Our intention is not to discuss this problem, but only to demonstrate that the EPI spectral function $\alpha^{2}(\omega) F(\omega)$, which is obtained from tunnelling experiments in cuprates (see Section 1.3.4), is able to explain the temperature dependence of $\rho_{a b}(T)$ in the optimally doped YBCO. In the Boltzmann theory $\rho_{a b}(T)$ is given by

$$
\rho_{a b}(T)=\rho_{\mathrm{imp}}+\frac{4 \pi}{\omega_{p}^{2}} \gamma_{\mathrm{tr}}(T)
$$

where

$$
\gamma_{\mathrm{tr}}(T)=\frac{\pi}{T} \int_{0}^{\infty} d \omega \frac{\omega}{\sinh ^{2}(\omega / 2 T)} \alpha_{\mathrm{tr}}^{2}(\omega) F(\omega) .
$$

The residual resistivity $\rho_{\text {imp }}$ is due to the impurity scattering. Since $\rho(T)=1 / \sigma(\omega=0, T)$ and having in mind that the dynamical conductivity $\sigma(\omega, T)$ in $Y B C O$ (at and near the optimal doping) is satisfactory explained by the EPI scattering, then it is to expect that $\rho_{a b}(T)$ is also dominated

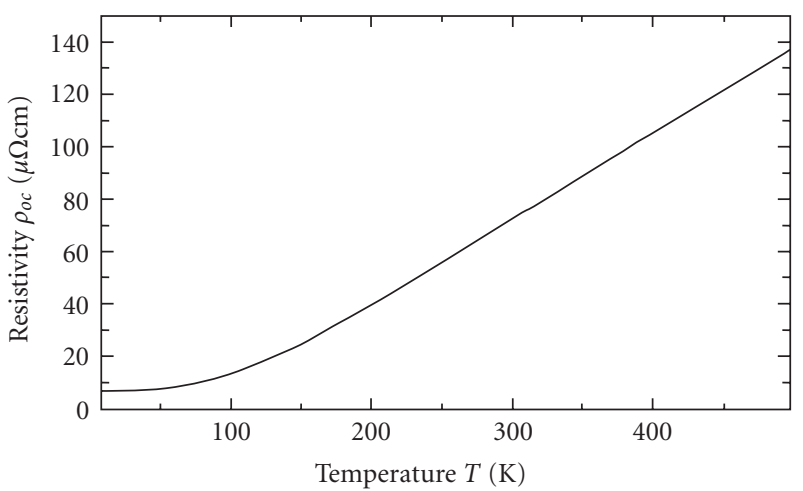

(a)

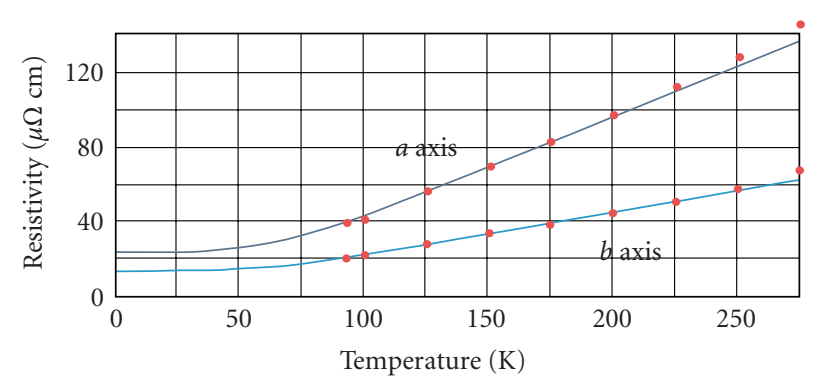

(b)

FIgure 12: (a) Calculated resistivity $\rho(T)$ for the EPI spectral function $\alpha_{\mathrm{tr}}^{2}(\omega) F(\omega)$ in [118]. (b) Measured resistivity in $a(x)$ and $b(y)$-crystal direction of YBCO [119] and calculated BlochGrüneisen curve (points) for $\lambda^{e p}=1[120]$.

by EPI in some temperature region $T>T_{c}$. This is indeed confirmed in the optimally doped $Y B C O$, where $\rho_{\text {imp }}$ is chosen appropriately and the spectral function $\alpha_{\mathrm{tr}}^{2}(\omega) F(\omega)$ is taken from the tunnelling experiments in [42-45]. The very good agreement with the experimental results [118] is shown in Figure 12. We stress that in the case of EPI there is always a temperature region where $\gamma_{\operatorname{tr}}(T) \sim T$ for $T>\alpha \Theta_{D}, \alpha<1$ depending on the shape of $\alpha_{\mathrm{tr}}^{2}(\omega) F(\omega)$ (for the simple Debye spectrum $\alpha \approx 0.2$ ). In the linear regime one has $\rho(T) \simeq$ $\rho_{\mathrm{imp}}+8 \pi^{2} \lambda_{e p, \operatorname{tr}}\left(k_{B} T / \hbar \omega_{p}^{2}\right)=\rho_{\mathrm{imp}}+\rho^{\prime} T$.

There is experimental constraint on $\lambda_{\text {tr }}$ since $\lambda_{\text {tr }} \approx$ $0.25 \omega_{\mathrm{pl}}^{2}(\mathrm{eV}) \rho^{\prime}(\mu \Omega \mathrm{cm} / \mathrm{K})$. For instance, for $\omega_{\mathrm{pl}} \approx(2-3) \mathrm{eV}$ [108] and $\rho^{\prime} \approx 0.6$ in the oriented YBCO films and $\rho^{\prime} \approx$ 0.3-0.4 in single crystals of BSCO, one obtains $\lambda_{\mathrm{tr}} \approx 0.6-1.4$. In case of YBCO single crystals, there is a pronounced anisotropy in $\rho_{a b}(T)$ [119] which gives $\rho_{x}^{\prime}(T)=0.6 \mu \Omega \mathrm{cm} / \mathrm{K}$ and $\rho_{y}^{\prime}(T)=0.25 \mu \Omega \mathrm{cm} / \mathrm{K}$. The function $\lambda_{\mathrm{tr}}\left(\omega_{\mathrm{pl}}\right)$ is shown in Figure 13 where the plasma frequency $\omega_{\text {pl }}$ can be calculated by LDA-DFT and also extracted from the width $\left(\sim \omega_{\mathrm{pl}}^{*}\right)$ of the Drude peak at small frequencies, where $\omega_{\mathrm{pl}}=\sqrt{\varepsilon_{\infty}} \omega_{\mathrm{pl}}^{*}$. We stress that the rather good agreement of theoretical and experimental results for $\rho_{a b}(T)$, in some optimally doped HTSC cuprates such as YBCO, should not be overinterpreted in the sense that the above rather simple electron-phonon approach can explain the resistivity in other HTSC cuprates and for various doping. For instance, in highly underdoped systems $\rho_{a b}(T)$ is very different from the behavior in Figure 12 and the simple Migdal-Eliashberg theory based 


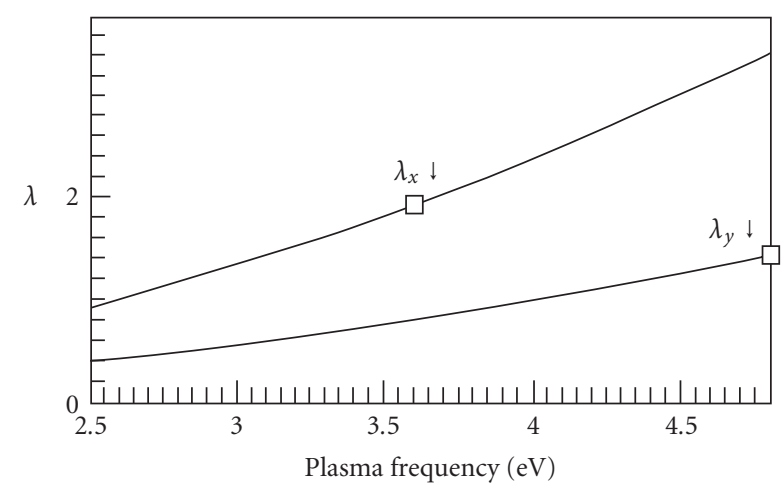

FIGURE 13: Transport EPI spectral function coupling constant in YBCO as a function of plasma frequency $\omega_{p}$ as derived from the experimental slope of resistivity $\rho^{\prime}(T) . \lambda_{x}$ for $\rho_{x}^{\prime}(T)=0.6 \mu \Omega \mathrm{cm} / \mathrm{K}$ and $\lambda_{y}$ for $\rho_{y}^{\prime}(T)=0.25 \mu \Omega \mathrm{cm} / \mathrm{K}$ [119]. Squares are LDA values [121].

on the EPI spectral function is inadequate. In this case one should certainly take into account polaronic effects [8-11], strong correlations, and so forth. The above analysis on the resistivity in the optimally doped YBCO demonstrates only that in this case if in (35) one uses the EPI spectral function $\alpha^{2}(\omega) F(\omega)$ obtained from the tunnelling experiments (and optics) one obtains the correct $T$ dependence of $\rho_{a, b}(T)$. This result is an additional evidence for the importance of EPI.

Concerning the temperature dependence of the resistivity in other (than YBCO) families of the optimally doped HTSC cuprates we would like to point out that there is some evidence that the linear (in $T$ ) resistivity is observed in some of them even at temperatures $T<0.2 \Theta_{D}[122$, 123]. This possibility is argued also theoretically in [124] where it is shown that in two-dimensional systems with a broad interval of phonon spectra the quasilinear behavior of $\rho_{a b}(T)$ is realized even at $T<0.2 \Theta_{D}$. The quasilinear behavior of the resistivity at $T \ll 0.2 \Theta_{D}$ has been observed in $\mathrm{Bi}_{2}\left(\mathrm{Sr}_{0.97} \mathrm{Pr}_{0.003}\right)_{2} \mathrm{CuO}_{6}$ [125], in LSCO, and in 1-layer $\mathrm{Bi}-2201[122,123,126,127]$, where in all these systems the critical temperature is rather small, $T_{c} \approx 10 \mathrm{~K}$. In that respect all existing theories based on the electronboson scattering are plagued and having difficulties to explain this low-temperature behavior of $\rho_{a b}(T)$. To this point, we would like to emphasize here that some of these (experimental) observations are contradictory. For example, the results obtained by the Vedeneev group [127] show that some samples demonstrate the quasilinear behavior of the resistivity up to $T=10 \mathrm{~K}$ but some others with approximately the same $T_{c}$ have the usual Bloch-Grüneisentype behavior characteristic for the EPI scattering. In that respect it is very unlikely that the linear resistivity up to $T=10 \mathrm{~K}$ can be simply explained in the standard way by interactions of electrons with some known bosons either by phonons or spin fluctuations (magnons). The question why in some cuprates the linear resistivity is observed up to $T=$ $10 \mathrm{~K}$ is still a mystery and its explanation is a challenge for all kinds of the electron-boson scattering, not only for EPI. In that respect it is interesting to mention that the existence of the forward scattering peak in EPI (with the width $q_{c} \ll k_{F}$ ), which is due to strong correlations, may give rise to the linear behavior of $\rho(T)$ down to very low temperatures $T \sim \Theta_{D} / 30$ $[6,128,129]$ - see more in Section 2.3.4, item (6). We will argue in Section 1.3.4 that if one interprets the tunnelling experiments in systems near optimal doping [42-54] in the framework of the Eliashberg theory one obtains the large EPI coupling constant $\lambda_{e p} \approx 2-3.5$ which implies that $\lambda_{\text {tr }} \sim(\lambda / 3)$. This means that EPI is reduced much more in transport properties than in the self-energy. We stress that such a large reduction of $\lambda_{\text {tr }}$ cannot be obtained within the LDA-DFT band-structure calculations, which means that $\lambda_{e p}$ and $\lambda_{\text {tr }}$ contain renormalization which do not enter in the LDA-DFT theory. In Section 2 we will argue that the strong suppression of $\lambda_{\text {tr }}$ may have its origin in strong electronic correlations $[78-80,130]$ and in the long-range Madelung energy [3-6].

(5) Femtosecond Time-Resolved Optical Spectroscopy. The femtosecond time-resolved optical spectroscopy (FTROS) has been developed in the last couple of years and applied to HTSC cuprates. In this method a femtosecond ( $1 \mathrm{fs}=$ $10^{-15} \mathrm{sec}$ ) laser pump excites in materials electron-hole pairs via interband transitions. These hot carriers release their energy via electron-electron (with the relaxation time $\left.\tau_{e e}\right)$ and electron-phonon scattering reaching states near the Fermi energy within 10-100 fs-see [131]. The typical energy density of the laser pump pulses with the wavelength $\lambda \approx 810 \mathrm{~nm}(\hbar \omega=1.5 \mathrm{eV})$ was around $F \sim 1 \mu \mathrm{J} / \mathrm{cm}^{2}$ (the excitation fluence $F$ ) which produces approximately $3 \times 10^{10}$ carriers per pulse (by assuming that each photon produces $\hbar \omega / \Delta$ carriers, $\Delta$ is the superconducting gap). By measuring photoinduced changes of the reflectivity in time, that is, $\Delta R(t) / R_{0}$, one can extract information on the relaxation dynamics of the low-laying electronic excitations. Since $\Delta R(t)$ relax to equilibrium, the fit with exponential functions is used as

$$
\frac{\Delta R(t)}{R_{0}}=f(t)\left[A e^{-t / \tau_{A}}+B e^{-t / \tau_{B}}+\cdots\right]
$$

where $f(t)=H(t)\left[1-\exp \left\{-t / \tau_{e e}\right\}\right](H(t)$ is the Heavyside function) describes the finite rise-time. The parameters $A, B$ depend on the fluence $F$. This method was used in studying the superconducting phase of $\mathrm{La}_{2-x} \mathrm{Sr}_{x} \mathrm{CuO}_{4}$, with $x=0.1$ and 0.15 and $T_{c}=30 \mathrm{~K}$ and $38 \mathrm{~K}$, respectively [41]. In that case one has $A \neq 0$ for $T<T_{c}$ and $A=0$ for $T>T_{c}$, while the signal $B$ was present also at $T>T_{c}$. It turns out that the signal $A$ is related to the quasiparticle recombination across the superconducting gap $\Delta(T)$ and has a relaxation time of the order $\tau_{A}>10 \mathrm{ps}$ at $T=4.5 \mathrm{~K}$. At the so called threshold fluence $\left(F_{T}=4.2 \pm 1.7 \mu \mathrm{J} / \mathrm{cm}^{2}\right.$ for $x=0.1$ and $F_{T}=5.8 \pm 2.3 \mu \mathrm{J} / \mathrm{cm}^{2}$ for $\left.x=0.15\right)$ the vaporization (destroying) of the superconducting phase occurs, where the parameter $A$ saturates. This vaporization process takes place at the time scala $\tau_{r} \approx 0.8 \mathrm{ps}$. The external fluence is distributed in the sample over the excitation volume which is proportional to the optical penetration depth $\lambda_{\mathrm{op}}(\approx 150 \mathrm{~nm}$ at $\lambda \approx 810 \mathrm{~nm}$ ) of the pump. The energy densities stored in the excitation volume at the vaporization threshold for $x=0.1$ and $x=0.15$ are $U_{p}=F_{T} / \lambda_{\mathrm{op}}=2.0 \pm 0.8 \mathrm{~K} / \mathrm{Cu}$ and $2.6 \pm 1.0 \mathrm{~K} / \mathrm{Cu}$, respectively. The important fact is that $U_{p}$ is 
much larger than the superconducting condensation energy which is $U_{\text {cond }} \approx 0.12 \mathrm{~K} / \mathrm{Cu}$ for $x=0.1$ and $U_{\text {cond }} \approx 0.3 \mathrm{~K} / \mathrm{Cu}$ for $x=0.15$, that is, $U_{p} \gg U_{\text {cond }}$. This means that the energy difference $U_{p}-U_{\text {cond }}$ must be stored elsewhere on the time scale $\tau_{r}$. The only present reservoir which can absorb the difference in energy is the bosonic baths of phonons and spin fluctuations. The energy required to heat the spin reservoir from $T=4.5 \mathrm{~K}$ to $T_{c}$ is $U_{\mathrm{sf}}=\int_{T}^{T_{c}} C_{\mathrm{sf}}(T) d T$. The measured specific heat $C_{\mathrm{sf}}(T)$ in $\mathrm{La}_{2} \mathrm{CuO}_{4}$ [41] gives very small value $U_{\text {sf }} \approx 0.01 \mathrm{~K}$. In the case of the phonon reservoir one obtains $U_{\mathrm{ph}}=\int_{T}^{T_{c}} C_{\mathrm{ph}}(T) d T=9 \mathrm{~K} / \mathrm{Cu}$ for $x=0.1$ and $28 \mathrm{~K} / \mathrm{Cu}$ for $x=0.15$, where $C_{\mathrm{ph}}$ is the phonon specific heat. Since $U_{\text {sf }} \ll U_{p}-U_{\text {cond }}$, the spin reservoir cannot absorb the rest energy $U_{p}-U_{\text {cond }}$. The situation is opposite with phonons since $U_{\mathrm{ph}} \gg U_{p}-U_{\text {cond }}$ and phonon can absorb the rest energy in the excitation volume. The complete vaporization dynamics can be described in the framework of the Rothwarf-Taylor model which describes approaching of electrons and phonons to quasiequilibrium on the time scale of 1 ps [132]. We will not go into details but only summarize by quoting the conclusion in [132] that only phonon-mediated vaporization is consistent with the experiments, thus ruling out spin-mediated quasiparticle recombination and pairing in HTSC cuprates. The FTROS method tells us that at least for nonequilibrium processes EPI is more important than SFI. It gives also some opportunities for obtaining the strength of EPI but at present there is no reliable analysis.

In conclusion, optics and resistivity measurements in the normal state of cuprates give evidence that EPI is important while the spin-fluctuation scattering is weaker than it is believed. However, some important questions related to the transport properties remain to be answered. (i) What are the values of $\lambda_{\mathrm{tr}}$ and $\omega_{\mathrm{pl}}$ ? (ii) What is the reason that $\lambda_{\text {tr }} \ll \lambda$ is realized in cuprates? (iii) What is the role of the Coulomb scattering in $\sigma(\omega)$ and $\rho(T)$ ? Later on we will argue that ARPES measurements in cuprates give evidence for an appreciable contribution of the Coulomb scattering at higher frequencies, where $\gamma(\omega) \approx \gamma_{0}+\lambda_{c} \omega$ for $\omega>\omega_{\max }^{\mathrm{ph}}$ with $\lambda_{c} \sim 1$. One should stress that despite the fact that EPI is suppressed in transport properties it is sufficiently strong in the quasiparticle self-energy, as it comes out from tunnelling measurements discussed below.

1.3.3. ARPES and the EPI Self-Energy. The angle-resolved photoemission spectroscopy (ARPES) is nowadays one of leading spectroscopy methods in the solid-state physics $[22,23]$. In some favorable conditions it provides direct information on the one-electron removal spectrum in a complex many-body system. The method involves shining light (photons) with energies between $E_{i}=5-1000 \mathrm{eV}$ on samples and by detecting momentum (k)- and energy $(\omega)$ distribution of the outgoing electrons. The resolution of ARPES has been significantly increased in the last decade with the energy resolution of $\Delta E \approx 1-2 \mathrm{meV}$ (for photon energies $\sim 20 \mathrm{eV}$ ) and angular resolution of $\Delta \theta \lesssim$ $0.2^{\circ}$. On the other side the ARPES method is surfacesensitive technique, since the average escape depth $\left(l_{\text {esc }}\right)$ of the outgoing electrons is of the order of $l_{\text {esc }} \sim 10 \AA$, depending on the energy of incoming photons. Therefore, very good surfaces are needed in order that the results be representative for bulk samples. The most reliable studies were done on the bilayer $\mathrm{Bi}_{2} \mathrm{Sr}_{2} \mathrm{CaCu}_{2} \mathrm{O}_{8}$ (Bi2212) and its single-layer counterpart $\mathrm{Bi}_{2} \mathrm{Sr}_{2} \mathrm{CuO}_{6}$ (Bi2201), since these materials contain weakly coupled $\mathrm{BiO}$ planes with the longest interplane separation in the cuprates. This results in a natural cleavage plane making these materials superior to others in ARPES experiments. After a drastic improvement of sample quality in other families of HTSC materials, the ARPES technique has became an important method in theoretical considerations. The ARPES can indirectly give information on the momentum and energy dependence of the pairing potential. Furthermore, the electronic spectrum of the (abovementioned) cuprates is highly quasi- $2 D$ which allows rather unambiguous determination of the initial state momentum from the measured final state momentum, since the component parallel to the surface is conserved in photoemission. In this case, the ARPES probes (under some favorable conditions) directly the single-particle spectral function $A(\mathbf{k}, \omega)$. In the following we discuss mainly those ARPES experiments which give evidence for the importance of the EPI in cuprates-see more in [22, 23].

ARPES measures a nonlinear response function of the electron system and it is usually analyzed in the so-called three-step model, where the total photoemission intensity $I_{\text {tot }}(\mathbf{k}, \omega) \approx I_{1} \cdot I_{2} \cdot I_{3}$ is the product of three independent terms: (1) $I_{1}$ that describes optical excitation of the electron in the bulk, (2) $I_{2}$ that describes the scattering probability of the travelling electrons, and (3) $I_{3}$ that describes the transmission probability through the surface potential barrier. The central quantity in the three-step model is $I_{1}(\mathbf{k}, \omega)$ and it turns out that for $\mathbf{k}=\mathbf{k}_{\|}$it can be written in the form $I_{1}(\mathbf{k}, \omega) \simeq I_{0}(\mathbf{k}, v) f(\omega) A(\mathbf{k}, \omega)[22,23]$ with $I_{0}(\mathbf{k}, v) \sim\left|\left\langle\psi_{f}|\mathbf{p A}| \psi_{i}\right\rangle\right|^{2}$ and the quasiparticle spectral function $A(\mathbf{k}, \omega)=-\operatorname{Im} G(\mathbf{k}, \omega) / \pi$ :

$$
A(\mathbf{k}, \omega)=-\frac{1}{\pi} \frac{\operatorname{Im} \Sigma(\mathbf{k}, \omega)}{[\omega-\xi(\mathbf{k})-\operatorname{Re} \Sigma(\mathbf{k}, \omega)]^{2}+\operatorname{Im} \Sigma^{2}(\mathbf{k}, \omega)} .
$$

Here, $\left\langle\psi_{f}|\mathbf{p} \cdot \mathbf{A}| \psi_{i}\right\rangle$ is the dipole matrix element which depends on $\mathbf{k}$, polarization, and energy $E_{i}$ of the incoming photons. The knowledge of the matrix element is of a great importance and its calculation from first principles was done in [133]. $f(\omega)$ is the Fermi function; $G$ and $\Sigma=\operatorname{Re} \Sigma+$ $i \operatorname{Im} \Sigma$ are the quasiparticle Green's function and the selfenergy, respectively. We summarize and comment here some important ARPES results which were obtained in the last several years and which confirm the existence of the Fermi surface and importance of EPI in the quasiparticle scattering $[22,23]$.

ARPES in the Normal State. (N1) There is well-defined Fermi surface in the metallic state of optimally and near optimally doped cuprates with the topology predicted by the LDA-DFT. However, the bands are narrower than LDA-DFT predicts which points to a strong quasiparticle 
renormalization. (N2) The spectral lines are broad with $|\operatorname{Im} \Sigma(\mathbf{k}, \omega)| \sim \omega($ or $\sim T$ for $T>\omega)$ which tells us that the quasiparticle liquid is a noncanonical Fermi liquid for larger values of $T, \omega$. (N3) There is a bilayer band splitting in Bi2212 (at least in the overdoped state), which is also predicted by LDA-DFT. In the case when the coherent hopping $t^{\perp}$ between two layers in the bilayer dominates, then the antibonding and bonding bands $\xi_{\mathrm{k}}^{a, b}=\xi_{\mathrm{k}} \pm t_{\mathrm{k}}^{\perp}$ with $t_{\mathrm{k}}^{\perp}=\left[t^{\perp}\left(\cos ^{2} k_{x}-\cos ^{2} k_{y}\right)+\right.$ ... ] have been observed. It is worth to mention that the previous experiments did not show this splitting which was one of the reasons for various speculations on some exotic electronic scattering and non-Fermi liquid scenarios. (N4) In the underdoped cuprates and at temperatures $T_{c}<T<T^{*}$ there is a $d$-wave-like pseudogap $\Delta_{p g}(\mathbf{k}) \sim \Delta_{p g, 0}\left(\cos k_{x}-\right.$ $\left.\cos k_{y}\right)$ in the quasiparticle spectrum where $\Delta_{p g, 0}$ increases by lowering doping. We stress that the pseudogap phenomenon is not well understood at present and since we are interested in systems near optimal doping where the pseudogap phenomena are absent or much less pronounced we will not discuss this problem here. Its origin can be due to a precursor superconductivity or due to a competing order, such as spin- or charge-density wave, strong correlations, and so forth. (N5) The ARPES self-energy gives evidence that EPI interaction is rather strong. The arguments for the latter statement are the following: (i) at $T>T_{c}$ there are kinks in the quasiparticle dispersion $\omega\left(\xi_{\mathrm{k}}\right)$ in the nodal direction (along the $(0,0)-(\pi, \pi)$ line) at the characteristic phonon energy $\omega_{\mathrm{ph}}^{(70)} \sim(60-70) \mathrm{meV}$ [91], see Figure 14 (top), and near the antinodal point $(\pi, 0)$ at $40 \mathrm{meV}[134]$ see Figure 14 (bottom).

(ii) The kink structure is observed in a variety of the hole-doped cuprates such as LSCO, Bi2212, Bi2201, T12201 $\left(\mathrm{Tl}_{2} \mathrm{Ba}_{2} \mathrm{CuO}_{6}\right), \mathrm{Na}-\mathrm{CCOC}\left(\mathrm{Ca}_{2-x} \mathrm{Na}_{x} \mathrm{CuO}_{2} \mathrm{Cl}_{2}\right)$. These kinks exist also above $T_{c}$, which excludes the scenario with the magnetic resonance peak in $\operatorname{Im} \chi_{s}(\mathbf{Q}, \omega)$. Moreover, since the tunnelling and magnetic neutron scattering measurements give small SFI coupling constant $g_{\text {sf }}<0.2 \mathrm{eV}$, then the kinks are not due to SFI. (iii) The position of the nodal kink is practically doping independent which points towards phonons as the scattering and pairing boson. (N6) The quasiparticles (holes) at and near the nodal-point $\mathbf{k}_{N}$ couple practically to a rather broad spectrum of phonons since at least three groups of phonons were extracted in the bosonic spectral function $\alpha^{2} F\left(\mathbf{k}_{N}, \omega\right)$ from the ARPES effective selfenergy in $\mathrm{La}_{2-x} \mathrm{Sr}_{x} \mathrm{CuO}_{4}$ [135]-Figure 15.

The latter result is in a qualitative agreement with numerous tunnelling measurements [42-54] which apparently demonstrate that the very broad spectrum of phonons couples with holes without preferring any particular phononssee discussion below. (N7) Recent ARPES measurements in Bi2212 [92] show very different slope $d \omega / d \xi_{\mathbf{k}}$ of the quasiparticle energy $\omega\left(\xi_{\mathrm{k}}\right)$ at small $\left|\xi_{\mathrm{k}}\right| \ll \omega_{\text {ph }}$ and at large energies $\left|\xi_{\mathbf{k}}\right| \gg \omega_{\mathrm{ph}}$-see Figure 16. The theoretical analysis [137] of these results gives the total coupling constant $\lambda^{Z}=$ $\lambda_{e p}^{Z}+\lambda_{c}^{Z} \approx 3$, and for the EPI coupling $\lambda_{e p}^{Z} \approx 2$, while the Coulomb coupling (SFI is a part of it) is $\lambda_{c}^{Z} \approx 1$ [137]see Figure 16. (Note that the upper index $\mathrm{Z}$ in the coupling constants means the quasiparticle renormalization in the normal part of the self-energy.) To this end let us mention some confusion which is related to the value of the EPI coupling constant extracted from ARPES. Namely, [22, 23, $138,139]$ the EPI self-energy was obtained by subtracting the high-energy slope of the quasiparticle spectrum $\omega\left(\xi_{k}\right)$ at $\omega \sim 0.3 \mathrm{eV}$. The latter is apparently due to the Coulomb interaction. Although the position of the low-energy kink is not affected by this procedure (if $\omega_{\mathrm{ph}}^{\max } \ll \omega_{c}$ ), this subtraction procedure gives in fact an effective EPI selfenergy $\Sigma_{\text {eff }}^{e p}(\mathbf{k}, \omega)$ and the effective coupling constant $\lambda_{e p \text {, eff }}^{Z}(\mathbf{k})$ only. We demonstrate below that the $\lambda_{e p \text {,eff }}^{Z}(\mathbf{k})$ is smaller than the real EPI coupling constant $\lambda_{e p}^{Z}(\mathbf{k})$. The total selfenergy is $\Sigma(\mathbf{k}, \omega)=\Sigma^{e p}(\mathbf{k}, \omega)+\Sigma^{c}(\mathbf{k}, \omega)$ where $\Sigma^{c}$ is the contribution due to the Coulomb interaction. At very low energies $\omega \ll \omega_{c}$ one has usually $\Sigma^{c}(\mathbf{k}, \omega)=-\lambda_{c}^{Z}(\mathbf{k}) \omega$, where $\omega_{c}(\sim 1 \mathrm{eV})$ is the characteristic Coulomb energies and $\lambda_{c}^{Z}$ is the Coulomb coupling constant. The quasiparticle spectrum $\omega(\mathbf{k})$ is determined from the condition $\omega-\xi(\mathbf{k})-$ $\operatorname{Re}\left[\Sigma^{e p}(\mathbf{k}, \omega)+\Sigma^{c}(\mathbf{k}, \omega)\right]=0$ where $\xi(\mathbf{k})$ is the bare bandstructure energy. At low energies $\omega<\omega_{\mathrm{ph}}^{\max } \ll \omega_{c}$ it can be rewritten in the form

$$
\omega-\xi^{\mathrm{ren}}(\mathbf{k})-\operatorname{Re} \Sigma_{\mathrm{eff}}^{e p}(\mathbf{k}, \omega)=0,
$$

with $\xi^{\mathrm{ren}}(\mathbf{k})=\left[1+\lambda_{c}^{Z}(\mathbf{k})\right]^{-1} \xi(\mathbf{k})$,

$$
\operatorname{Re} \Sigma_{\mathrm{eff}}^{e p}(\mathbf{k}, \omega)=\frac{\operatorname{Re} \Sigma_{\mathrm{eff}}^{e p}(\mathbf{k}, \omega)}{1+\lambda_{c}^{Z}(\mathbf{k})} .
$$

Since at very low energies $\omega \ll \omega_{\mathrm{ph}}^{\max }$ one has $\operatorname{Re} \Sigma^{e p}(\mathbf{k}, \omega)=$ $-\lambda_{e p}^{Z}(\mathbf{k}) \omega$ and $\operatorname{Re} \Sigma_{\text {eff }}^{e p}(\mathbf{k}, \omega)=-\lambda_{e p \text {,eff }}^{Z}(\mathbf{k}) \omega$, then the real coupling constant is related to the effective one by

$$
\lambda_{e p}^{Z}(\mathbf{k})=\left[1+\lambda_{c}^{Z}(\mathbf{k})\right] \lambda_{e p, \mathrm{eff}}^{Z}(\mathbf{k}) .
$$

As a result one has $\lambda_{e p}^{Z}(\mathbf{k})>\lambda_{e p \text {,eff }}^{Z}(\mathbf{k})$. At higher energies $\omega_{\mathrm{ph}}^{\max }<\omega<\omega_{c}$, where the EPI effects are suppressed and $\Sigma^{e p}(\mathbf{k}, \omega)$ stops growing, one has $\operatorname{Re} \Sigma(\mathbf{k}, \omega) \approx \operatorname{Re} \Sigma^{e p}(\mathbf{k}, \omega)-$ $\lambda_{c}^{Z}(\mathbf{k}) \omega$. The measured $\operatorname{Re} \Sigma^{\exp }(\mathbf{k}, \omega)$ at $T=10 \mathrm{~K}$ near and slightly away from the nodal point in the optimally doped Bi2212 with $T_{c}=91 \mathrm{~K}[136]$ is shown in Figure 16.

It is seen that $\operatorname{Re} \Sigma^{\exp }(\mathbf{k}, \omega)$ has two kinks-the first one at low energy $\omega_{1} \approx \omega_{\mathrm{ph}}^{\text {high }} \approx 50-70 \mathrm{meV}$ which is (as we already argued) most probably of the phononic origin $[22,23,138,139]$, while the second kink at higher energy $\omega_{2} \approx \omega_{c} \approx 350 \mathrm{meV}$ which is due to the Coulomb interaction. However, the important results in [136] are that the slopes of $\operatorname{Re} \Sigma^{\exp }(\mathbf{k}, \omega)$ at low $\left(\omega<\omega_{\mathrm{ph}}^{\text {high }}\right)$ and high energies $\left(\omega_{\mathrm{ph}}^{\text {high }}<\omega<\omega_{c}\right)$ are very different. The lowenergy and high-energy slope near the nodal point are shown in Figure 16 schematically (thin lines). From Figure 16 it is obvious that EPI prevails at low energies $\omega<\omega_{\mathrm{ph}}^{\text {high }}$. More precisely digitalization of $\operatorname{Re} \Sigma^{\exp }(\mathbf{k}, \omega)$ in the interval $\omega_{\text {ph }}^{\text {high }}<$ $\omega<0.4 \mathrm{eV}$ gives the Coulomb coupling $\lambda_{c}^{Z} \approx 1.1$ while the same procedure at $20 \mathrm{meV} \approx \omega_{\mathrm{ph}}^{\text {low }}<\omega<\omega_{\mathrm{ph}}^{\text {high }} \approx$ $50-70 \mathrm{meV}$ gives the total coupling constant $\left(\lambda_{2} \equiv\right) \lambda^{Z}=$ $\lambda_{e p}^{Z}+\lambda_{c}^{Z} \approx 3.2$ and the EPI coupling constant $\lambda_{e p}^{Z}\left(\equiv \lambda_{e p, \text { high }}^{Z}\right) \approx$ 

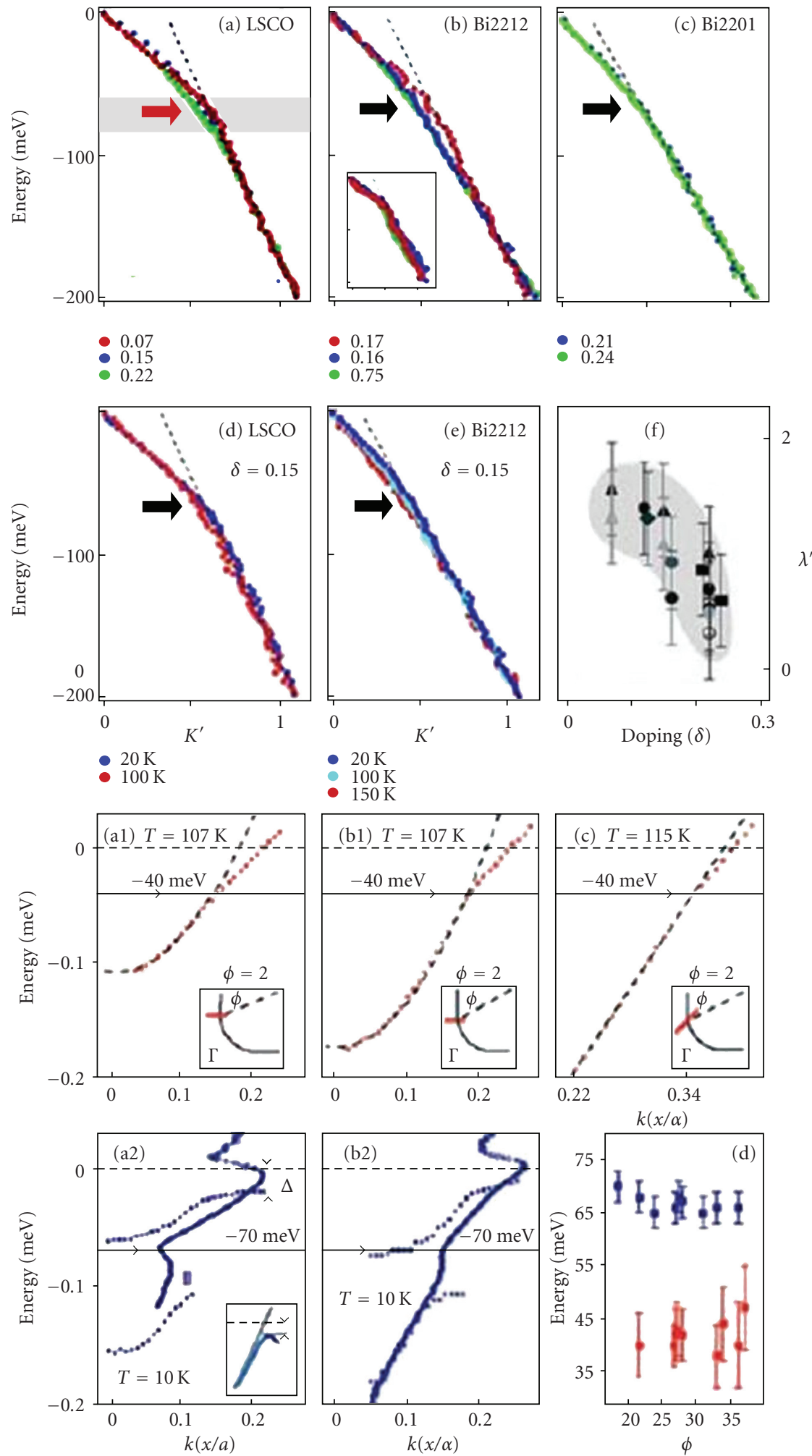

FIGURE 14: (top) Quasiparticle dispersion of Bi2212, Bi2201, and LSCO along the nodal direction, plotted versus the momentum $k$ for (a)-(c) different doping, and (d)-(e) different $T$; black arrows indicate the kink energy; the red arrow indicates the energy of the $q=(\pi, 0)$ oxygen stretching phonon mode; inset of (e) shows T-dependent $\Sigma^{\prime}$ for optimally doped Bi2212; (f) shows doping dependence of the effective coupling constant $\lambda^{\prime}$ along $(0,0)-(\pi, \pi)$ for the different HTSC oxides. From [91]. (bottom) Quasiparticle dispersion $E(k)$ in the normal state $(\mathrm{a} 1, \mathrm{~b} 1, \mathrm{c})$, at $107 \mathrm{~K}$ and $115 \mathrm{~K}$, along various directions $\phi$ around the antinodal point. The kink at $E=40 \mathrm{meV}$ is shown by the horizontal arrow. (a2 and b2) are $E(k)$ in the superconducting state at $10 \mathrm{~K}$ with the shifted kink to $70 \mathrm{meV}$. (d) kink positions as a function of $\phi$ in the antinodal region. From [134]. 


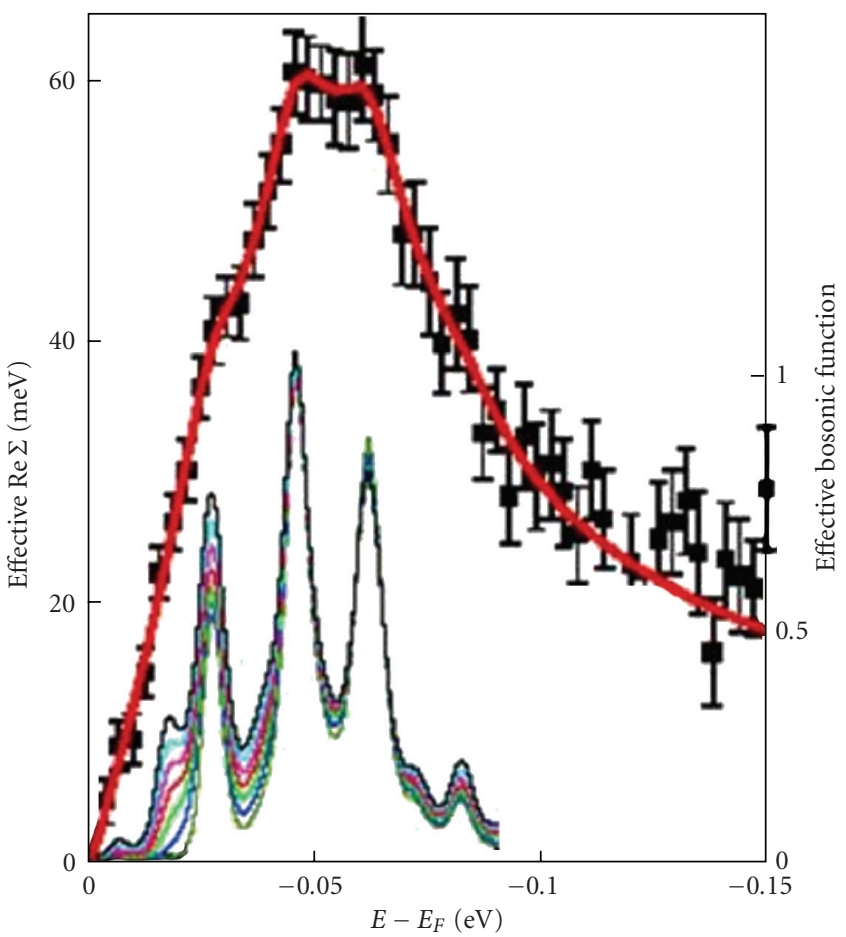

(a)

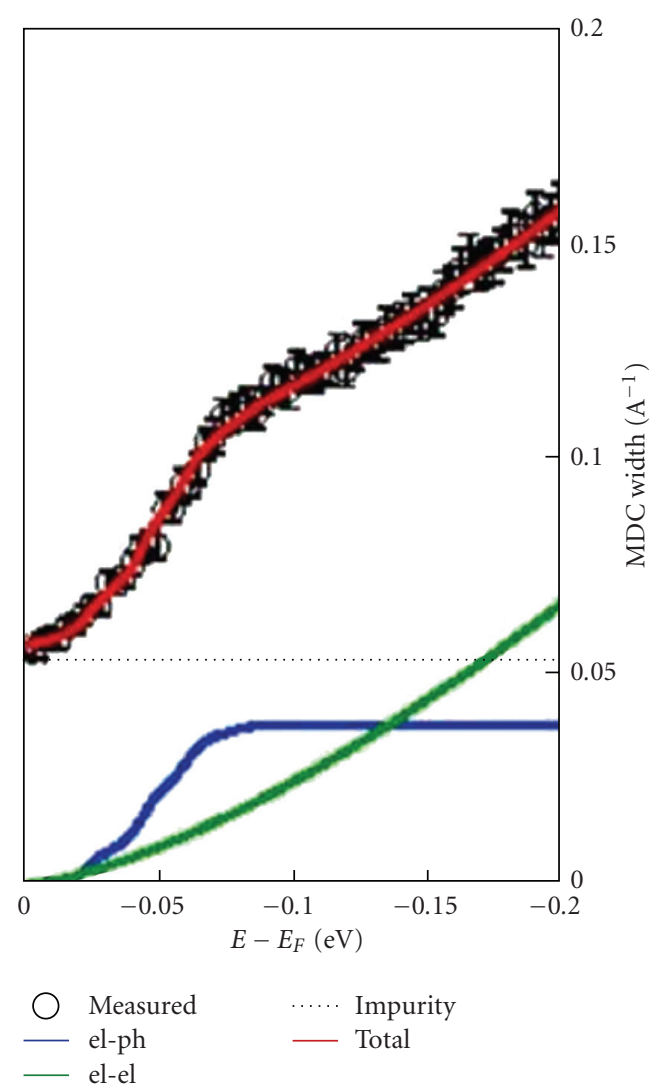

(b)

FIGURE 15: (a) Effective real self-energy for the nonsuperconducting $\mathrm{La}_{2-x} \mathrm{Sr}_{x} \mathrm{CuO}_{4}, x=0.03$. Extracted $\alpha_{\text {eff }}^{2}(\omega) F(\omega)$ is in the inset. (b) Top: the total MDC width-open circles. Bottom: the EPI contribution shows saturation, impurity contribution-dotted black line. The residual part is growing $\sim \omega^{1.3}$. From [135].

$2.1>2 \lambda_{e p, \text { eff }}^{Z}(\mathbf{k})$, that is, the EPI coupling is at least twice larger than the effective EPI coupling constant obtained in the previous analysis of ARPES results [22, 23, 138, 139]. This estimation tells us that at (and near) the nodal point, the EPI interaction dominates in the quasiparticle scattering at low energies since $\lambda_{e p}^{Z}(\approx 2.1) \approx 2 \lambda_{z}^{c}>2 \lambda_{\mathrm{sf}}^{Z}$, while at large energies (compared to $\omega_{\mathrm{ph}}$ ) the Coulomb interaction with $\lambda_{c}^{Z} \approx 1.1$ dominates. We point out that EPI near the antinodal point can be even larger than in the nodal point, mostly due to the higher density of states near the antinodal point. (N8) Recent ARPES spectra in the optimally doped Bi2212 near the nodal and antinodal point [139] show a low-energy isotope effect in $\operatorname{Re} \Sigma^{\exp }(\mathbf{k}, \omega)$, which can be well described in the framework of the Migdal-Eliashberg theory for EPI [140]. At higher energies $\omega>\omega_{\text {ph }}$ obtained in [139] very pronounced isotope effect cannot be explained by the simple Migdal-Eliashberg theory [140]. However, there are controversies with the strength of the high-energy isotope effect since it was not confirmed in other measurements $[141,142]$ - see the discussion in Section 1.3.6(2) related to the isotope effects in HTSC cuprates. (N9) The ARPES experiments in $\mathrm{Ca}_{2} \mathrm{CuO}_{2} \mathrm{Cl}_{2}$ give strong evidence for the formation of small polarons in undoped cuprates which are due to phonons and strong EPI, while in the doped systems quasiparticles are formed and there are no small polarons [143]. Namely, in [143] a broad peak around $-0.8 \mathrm{eV}$ is observed at the top of the band $(\mathbf{k}=(\pi / 2, \pi / 2))$ with the dispersion similar to that predicted by the $t-J$ model - see Figure 17.

However, the peak in Figure 17(a) is of Gaussian shape and can be described only by coupling to bosons, that is, this peak is a boson side band-see more in $[10,11]$ and references therein. The theory based on the $t-J$ model (in the antiferromagnetic state of the undoped compound) by including coupling to several (half-breathing, apical oxygen, low-lying) phonons, which is given in [144-146], explains successfully this broad peak of the boson side band by the formation of small polarons due to the EPI coupling $\left(\lambda_{e p} \approx\right.$ 1.2). Note that this value of $\lambda_{e p}$ is for the polaron at the bottom of the band while in the case where the Fermi surface exists (in doped systems) this coupling is even larger due to the larger density of states at the Fermi surface [144-146]. In [144-146] it was stressed that even when the electronmagnon interaction is stronger than EPI the polarons in the undoped systems are formed due to EPI. The latter mechanism involves excitation of many phonons at the lattice site (where the hole is seating), while it is possible to excite only one magnon at the given site. (N10) Recent 


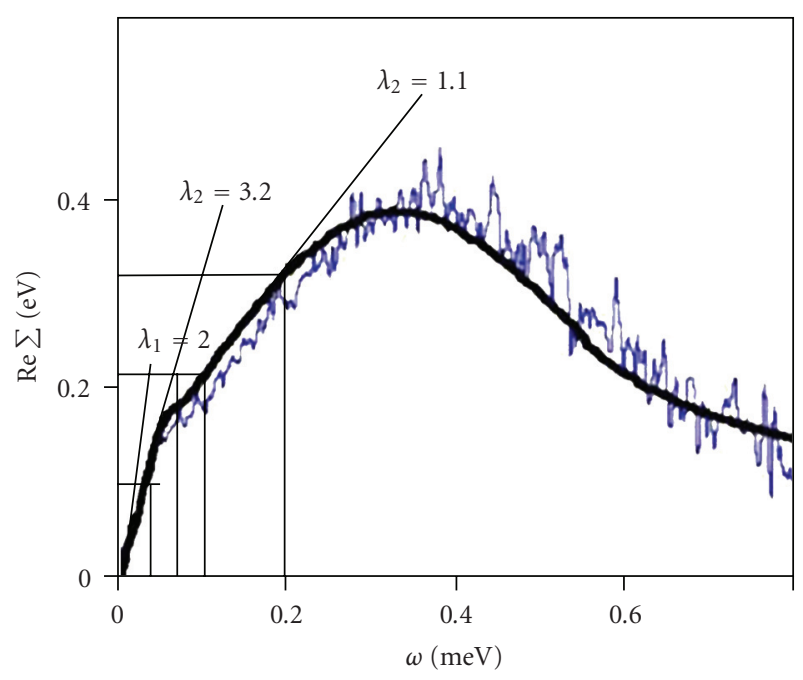

FIGURE 16: Figure 4b from [136]: Re $\Sigma(\omega)$ measured in Bi2212 (thin line) and model $\operatorname{Re} \Sigma(\omega)$ (bold line) obtained in [136]. The three thin lines $\left(\lambda_{1}, \lambda_{2}, \lambda_{3}\right)$ are the slopes of $\operatorname{Re} \Sigma(\omega)$ in different energy regions - see the text.

soft X-ray ARPES measurements on the electron-doped HTSC $\mathrm{Nd}_{1.85} \mathrm{Ce}_{0.15} \mathrm{CuO}_{4}$ [147], and $\mathrm{Sm}_{(2-x)} \mathrm{Ce}_{x} \mathrm{CuO}_{4}(x=$ 0.1, 0.15, 0.18), $\mathrm{Nd}_{1.85} \mathrm{Ce}_{0.15} \mathrm{CuO}_{4}$, and $\mathrm{Eu}_{1.85} \mathrm{Ce}_{0.15} \mathrm{CuO}_{4}$ [148] show kink at energies $50-70 \mathrm{meV}$ in the quasiparticle dispersion relation along both the nodal and antinodal, directions as it is shown in Figure 18.

It is seen from this figure that the effective EPI coupling constant $\lambda_{e p \text {, eff }}\left(<\lambda_{e p}\right)$ is isotropic and $\lambda_{e p \text {,eff }} \approx 0.8-1$. It seems that the kink in the electron-doped cuprates is due solely to EPI and in that respect the situation is similar to the one in the hole-doped cuprates.

ARPES Results in the Superconducting State. (S1) There is an anisotropic superconducting gap in most HTSC compounds $[22,23]$, which is predominately $d$-wave like, that is, $\Delta(\mathbf{k}) \approx$ $\Delta_{0}\left(\cos k_{x}-\cos k_{y}\right)$ with $2 \Delta_{0} / T_{c} \approx 5-6$ in the optimally doped systems. (S2) The particle-hole coherence in the superconducting state which is expected for the BCS-like theory of superconductivity has been observed first in [149] and confirmed with better resolution in [150], where the particle-hole mixing is clearly seen in the electron and hole quasiparticle dispersion. To remind the reader, the excited Bogoliubov-Valatin quasiparticles $\left(\hat{\alpha}_{\mathbf{k}, \pm}\right)$ with energies $E_{\mathbf{k}}^{\alpha_{ \pm}}=$ $\sqrt{\xi_{\mathbf{k}}^{2}+\left|\Delta_{\mathbf{k}}\right|^{2}}$ are a mixture of electron $\left(\hat{c}_{\mathbf{k}, \sigma}\right)$ and hole $\left(\hat{c}_{-\mathbf{k},-\sigma}^{\dagger}\right)$, that is, $\hat{\alpha}_{\mathbf{k},+}=u_{\mathbf{k}} \hat{c}_{\mathbf{k} \uparrow}+v_{\mathbf{k}} \hat{c}_{-\mathbf{k} \downarrow}^{\dagger}, \hat{\alpha}_{\mathbf{k},-}=u_{\mathbf{k}} \hat{c}_{-\mathbf{k} \downarrow}+v_{\mathbf{k}} \hat{c}_{\mathbf{k} \uparrow}^{\dagger}$ where the coherence factors $u_{\mathrm{k}}, v_{\mathrm{k}}$ are given by $\left|u_{\mathrm{k}}\right|^{2}=1-\left|v_{\mathrm{k}}\right|^{2}=$ $\left(1+\xi_{\mathrm{k}} / E_{\mathrm{k}}\right) / 2$. Note that $\left|u_{\mathrm{k}}\right|^{2}+\left|v_{\mathrm{k}}\right|^{2}=1$, which is exactly observed, together with $d$-wave pairing $\Delta(\mathbf{k})=\Delta_{0}\left(\cos k_{x}-\right.$ $\left.\cos k_{y}\right)$, in experiments in [150]. This is very important result since it proves that the pairing in HTSC cuprates is of the BCS type and not exotic one as it was speculated long time after the discovery of HTSC cuprates. (S3) The kink at (60-70) $\mathrm{meV}$ in the quasiparticle energy around the nodal point is not shifted (in energy) while the antinodal kink at $\omega_{\mathrm{ph}}^{(40)} \sim 40 \mathrm{meV}$ is shifted (in energy) in the superconducting state by $\Delta_{0}(=(25-30) \mathrm{meV})$, that is, $\omega_{\mathrm{ph}}^{(40)} \rightarrow \omega_{\mathrm{ph}}^{(40)}+\Delta_{0}=$ $(65-70) \mathrm{meV}[22,23]$. To remind the reader, in the standard Eliashberg theory the kink in the normal state at $\omega=\omega_{\text {ph }}$ should be shifted in the superconducting state to $\omega_{\mathrm{ph}}+\Delta_{0}$ at all k-points at the Fermi surface. This puzzling result (that the quasiparticle energy around the nodal point is not shifted in the superconducting state) might be a smoking gun result since it makes an additional constraint on the quasiparticle interaction in cuprates. Until now there is only one plausible explanation [151] of this nonshift puzzle which is based on an assumption of the forward scattering peak (FSP) in EPI-see more in Section 2. The FSP in EPI means that electrons scatter into a narrow region $\left(q<q_{c} \ll k_{F}\right)$ around the initial point in the $k$-space, so that at the most part of the Fermi surface there is practically no mixing of states with different signs of the order parameter $\Delta(\mathbf{k})$. In that case the EPI bosonic spectral function (which is defined in Appendix A) $\alpha^{2} F\left(\mathbf{k}, \mathbf{k}^{\prime}, \Omega\right) \approx \alpha^{2} F\left(\varphi, \varphi^{\prime}, \Omega\right)(\varphi$ is the angle on the Fermi surface) has a pronounced forward scattering peak (at $\delta \varphi=\varphi-\varphi^{\prime}=0$ ) due to strong correlationssee Section 2. Its width $\delta \varphi_{c}$ is narrow, that is, $\delta \varphi_{c} \ll 2 \pi$ and the angle integration goes over the region $\delta \varphi_{c}$ around the point $\varphi$. In that case the kink is shifted (approximately) by the local gap $\Delta(\varphi)=\Delta_{\max } \cos 2 \varphi$-for more details see [151]. As a consequence, the antinodal kink is shifted by the maximal gap, that is, $\left|\Delta\left(\varphi_{\mathrm{AN}} \approx \pi / 2\right)\right|=\Delta_{\max }$ while the nodal gap is practically unshifted since $\left|\Delta\left(\varphi_{\mathrm{AN}} \approx \pi / 4\right)\right| \approx 0$. (S4) The recent ARPES spectra [152] in the undoped single crystalline 4-layered cuprate with the apical fluorine $(\mathrm{F})$, $\mathrm{Ba}_{2} \mathrm{Ca}_{3} \mathrm{Cu}_{4} \mathrm{O}_{8} \mathrm{~F}_{2}$ (F0234) give rather convincing evidence against the SFI mechanism of pairing — see Figure 19.

First, F0234 is not a Mott insulator-as expected from valence charge counting which puts $\mathrm{Cu}$ valence as $2^{+}$, but it is a superconductor with $T_{c}=60 \mathrm{~K}$. Moreover, the ARPES data [152] reveal at least two metallic Fermi-surface sheets with corresponding volumes equally below and above halffilling-see Figure 20.

Second, one of the Fermi surfaces is due to the electronlike $(N)$ band (with $20 \pm 6 \%$ electron-doping) and the other one due to the hole-like $(P)$ band (with $20 \pm 8 \%$ hole-doping) and their splitting along the nodal direction is significant and cannot be explained by the LDA-DFT calculations [153]. This electron and hole self-doping of inner and outer layers is in an appreciable contrast to other multilayered cuprates where there is only hole selfdoping. For instance, in $\mathrm{HgBa}_{2} \mathrm{Ca}_{n} \mathrm{Cu}_{n+1} \mathrm{O}_{2 n+2}(n=2,3)$ and $(\mathrm{Cu}, \mathrm{C}) \mathrm{Ba}_{2} \mathrm{Ca}_{n} \mathrm{Cu}_{n+1} \mathrm{O}_{3 n+2}(n=2,3,4)$, the inner $\mathrm{CuO}_{2}$ layers are less hole-doped than outer layers. It turns out, unexpectedly, that the superconducting gap on the N-band Fermi surface is significantly larger than on the $P$-one, where in $\mathrm{Ba}_{2} \mathrm{Ca}_{3} \mathrm{Cu}_{4} \mathrm{O}_{8} \mathrm{~F}_{2}$ the ratio is anomalous $\left(\Delta_{N} / \Delta_{P}\right) \approx$ 2 and $\Delta_{N}$ is an order of magnitude larger than in the electron-doped cuprate $\mathrm{Nd}_{2-x} \mathrm{Ce}_{x} \mathrm{CuO}_{4}$. Third, the $N$-band Fermi surface is rather far from the antinodal point at $(\pi, 0)$. This is very important result which means that the antiferromagnetic spin fluctuations with the AF wave-vector $\mathbf{Q}=(\pi, \pi)$, as well as the van Hove singularity, are not dominant in the pairing in the $N$-band. To remind the reader, the SFI scenario assumes that the pairing is due to spin 


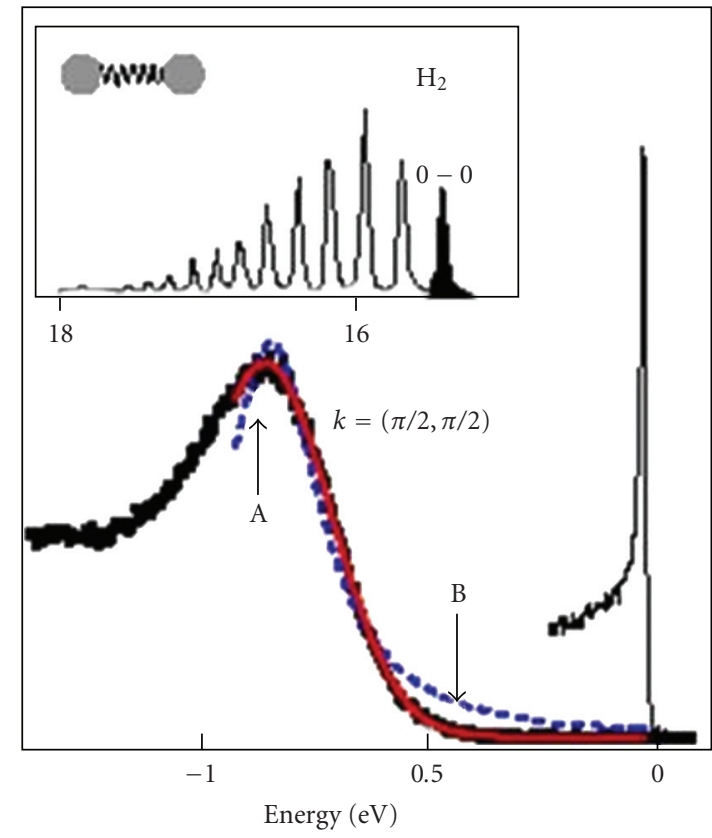

(a)
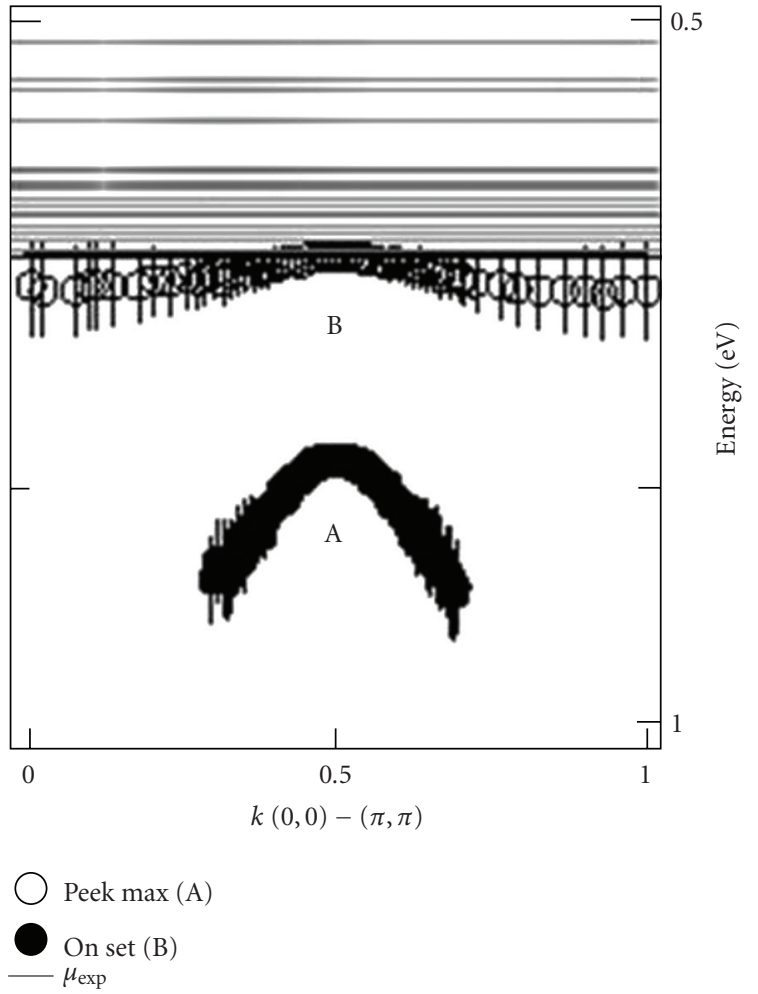

Figure 17: (a) The ARPES spectrum of undoped $\mathrm{Ca}_{2} \mathrm{CuO}_{2} \mathrm{Cl}_{2}$ at $\mathbf{k}=(\pi / 2, \pi / 2)$. Gaussian shape—solid line, Lorentzian shape-dashed line. (b) Dispersion of the polaronic band-A - and of the quasiparticle band-B-along the nodal direction. Horizontal lines are the chemical potentials for a large number of samples. From [143].

fluctuations with the wave-vector $\mathbf{Q}$ (and near it) which connects two antinodal points which are near the van Hove singularity at the hole-surface $($ at $(\pi, 0)$ and $(0, \pi))$ giving rise to large density of states. This is apparently not the case for the N-band Fermi surface-see Figure 20. The ARPES data give further that there is a kink at $\sim 85 \mathrm{meV}$ in the quasiparticle dispersion of both bands, while the kink in the $N$-band is stronger than that in the $P$-band. This result, together with the anomalous ratio $\left(\Delta_{N} / \Delta_{P}\right) \approx 2$, disfavors SFI as a pairing mechanism. (S5) Despite the presence of significant elastic quasiparticle scattering in a number of samples of optimally doped Bi-2212, there are dramatic sharpenings of the spectral function near the antinodal point $(\pi, 0)$ at $T<T_{c}$ (in the superconducting state) [154]. This effect can be explained by assuming that the small $q$ scattering (the forward scattering peak) dominates in the elastic impurity scattering as it is pointed in [78-80, 130, $155,156]$. As a result, one finds that the impurity scattering rate in the superconducting state is almost zero, that is, $\gamma_{\operatorname{imp}}(\mathbf{k}, \omega)=\gamma_{n}(\mathbf{k}, \omega)+\gamma_{a}(\mathbf{k}, \omega) \approx 0$ for $|\omega|<\Delta_{0}$ for any kind of pairing $\left(s^{-}, p^{-}, d\right.$-wave, etc.) since the normal $\left(\gamma_{n}\right)$ and the anomalous $\left(\gamma_{a}\right)$ scattering rates compensate each other. This collapse of the elastic scattering rate is elaborated in details in [154] and it is a consequence of the Andersonlike theorem for unconventional superconductors which is due to the dominance of the small $q$-scattering [78-80, 130, $155,156]$. In such a case $d$-wave pairing is weakly unaffected by nonmagnetic impurities and as a consequence there is small reduction in $T_{c}[156,157]$. The physics behind this result is rather simple. The small $q$-scattering (usually called forward scattering) means that electrons scatter into a small region in the $k$-space, so that at the most part of the Fermi surface there is no mixing of states with different signs of the order parameter $\Delta(\mathbf{k})$. In such a way the detrimental effect of nonmagnetic impurities on $d$-wave pairing is significantly reduced. This result points to the importance of strong correlations in the renormalization of the nonmagnetic impurity scattering too-see discussion in Section 2.

In conclusion, in order to explain the ARPES results in cuprates it is necessary to take into account (1) the electron-phonon interaction (EPI) since it dominates in the quasiparticle scattering in the energy region important for pairing, (2) the elastic nonmagnetic impurities with the forward scattering peak (FSP) due to strong correlations, and (3) the Coulomb interaction which dominates at higher energies $\omega>\omega_{\mathrm{ph}}$. In this respect, the presence of ARPES kinks and the knee-like shape of the $T$ dependence of the spectral width are important constraints on the scattering and pairing mechanism in HTSC cuprates.

1.3.4. Tunnelling Spectroscopy and Spectral Function $\alpha^{2} F(\omega)$. By measuring current-voltage $I-V$ characteristics in NIS (normal metal-insulator-superconductor) tunnelling junctions with large tunnelling barrier one obtains from tunnelling conductance $G_{\mathrm{NS}}(V)=d I / d V$ the so called tunnelling density of states in superconductors $N_{T}(\omega)$. 


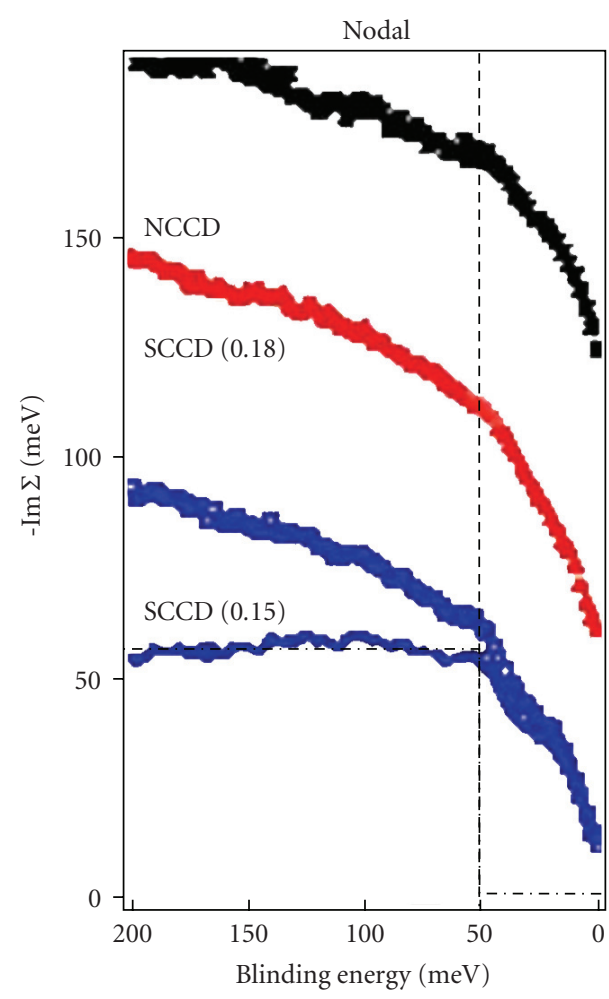

(a)

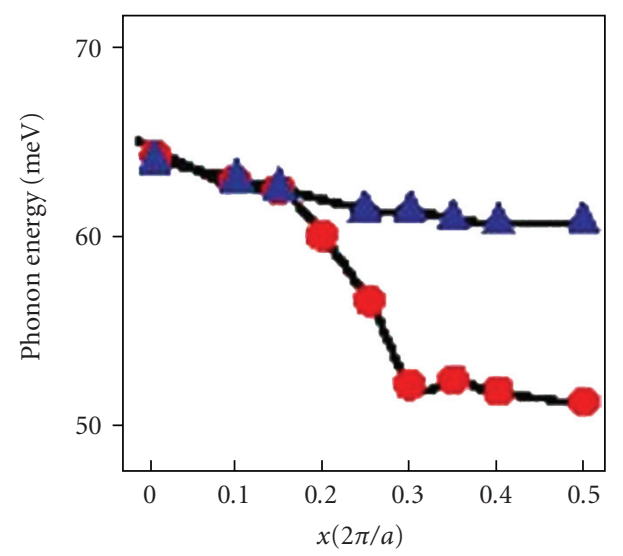

(c)

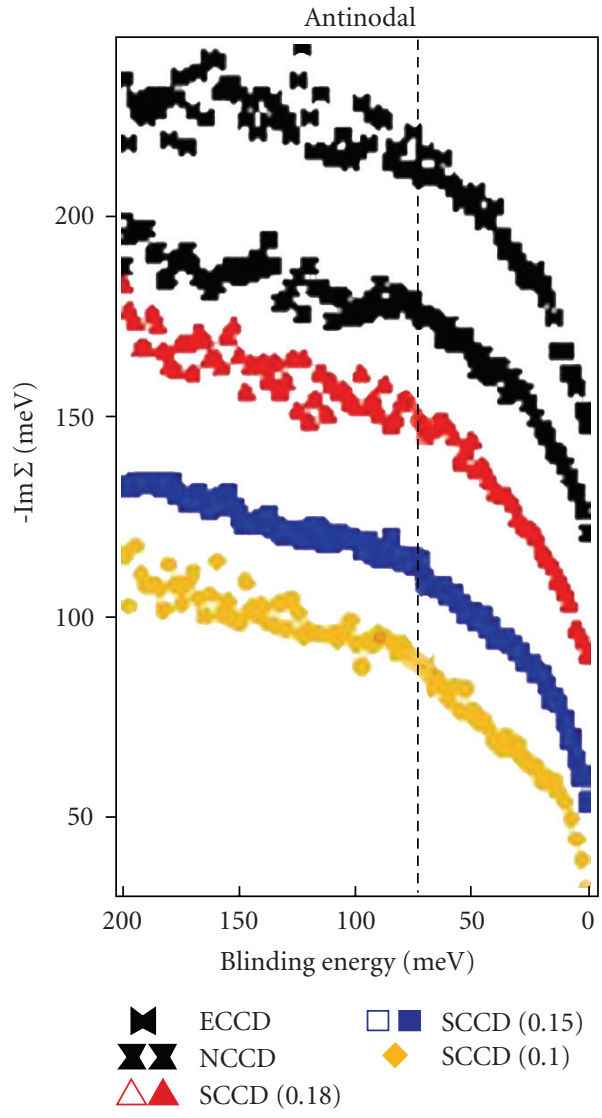

(b)

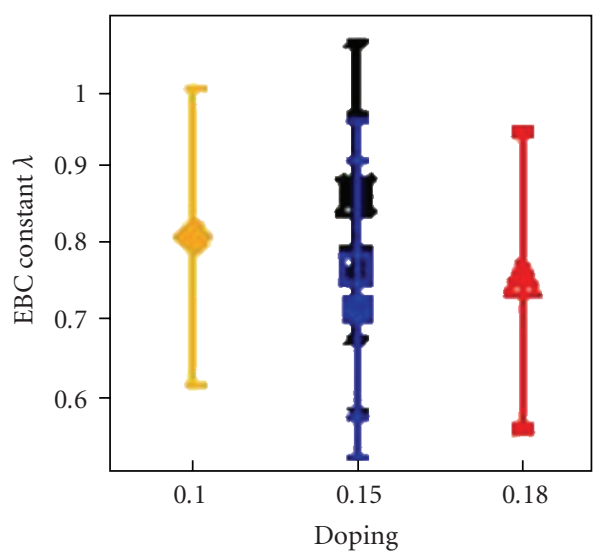

(d)

FIGURE 18: NCCO electron-doped: (a) $\operatorname{Im} \Sigma(\omega)$ measured in the nodal point. Curves are offsets by 50 meV for clarity. The change of the slope in the last bottom curve is at the phonon energy. (b) $\operatorname{Im} \Sigma(\omega)$ for the antinodal direction with 30 meV offset. (c) Experimental phonon dispersion of the bond stretching modes. (d) Estimated $\lambda_{\text {eff }}^{e p}$ from $\operatorname{Im} \Sigma(\omega)$. From [148].

Moreover, by measuring of $G_{\mathrm{NS}}(V)$ at voltages $\mathrm{eV}>\Delta$ it is possible to determine the Eliashberg spectral function $\alpha^{2} F(\omega)$ and finally to confirm the phonon mechanism of pairing in LTSC materials. Four tunnelling techniques were used in the study of HTSC cuprates: (1) vacuum tunnelling by using the $S T M$ technique-scanning tunnelling microscope; (2) point-contact tunnelling; (3) break-junction tunnelling; (4) planar-junction tunnelling. Each of these techniques has some advantages although in principle the most potential one is the STM technique since it measures superconducting properties locally [158]. Since tunnelling measurements probe a surface region on the scale of the superconducting coherence length $\xi_{0}$, then this kind of measurements in HTSC materials with small coherence length $\xi_{0}\left(\xi_{a b} \sim 20 \AA\right.$ in the $a-b$ plane and $\xi_{c} \sim 1-3 \AA$ along the $c$-axis) depends strongly on the surface quality 


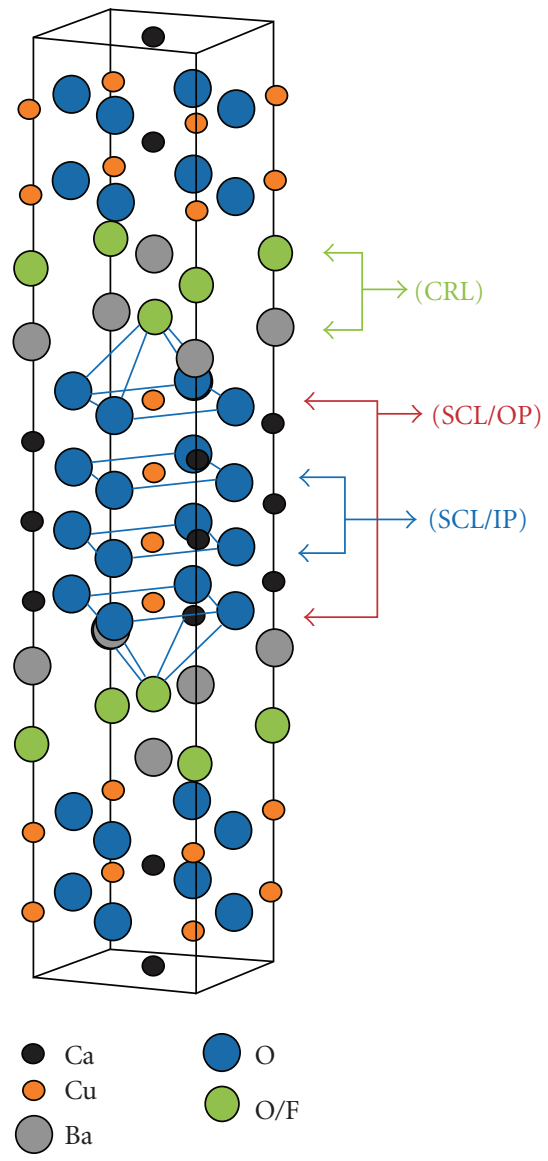

Figure 19: Crystal structure of $\mathrm{Ba}_{2} \mathrm{Ca}_{3} \mathrm{Cu}_{4} \mathrm{O}_{8}\left(\mathrm{O}_{\delta} \mathrm{F}_{1-\delta}\right)_{2}$. There are four $\mathrm{CuO}_{2}$ layers in a unit cell with the outer having apical $\mathrm{F}$ atoms. CRL — charge reservoir layer; SCL/OP_superconducting layer/outer plane; SCL/IP-superconducting layer/inner plane. From [152].

and sample preparation. Nowadays, many of the material problems in HTSC cuprates are understood and as a result consistent picture of tunnelling features is starting to emerge.

From tunnelling experiments one obtains the (energydependent) gap function $\Delta(\omega)$ in the superconducting state. Since we have already discussed this problem in [6], we will only briefly mention some important result. For instance, in most systems $G_{\mathrm{NS}}(V)$ has $V$-shape in all families of HTSC hole- and electron-doped cuprates. The $V$-shape is characteristic for $d$-wave pairing with gapless spectrum, which is also confirmed in the interference experiments on hole- and electron-doped cuprates [75]. Some experiments give a $U$-shape of $G_{\mathrm{NS}}(V)$ which resembles $s$-wave pairing. This controversy is explained to be the property of the tunnelling matrix element which filters out states with the maximal gap.

Here we are interested in the bosonic spectral function $\alpha^{2} F(\omega)$ of HTSC cuprates near optimal doping which can be extracted by using tunnelling spectroscopy. We inform the reader in advance that the shape and the energy width of $\alpha^{2} F(\omega)$, which are extracted from the second derivative $d^{2} I / d V^{2}$ at voltages above the superconducting gap, in most HTSC cuprates resemble the phonon density of states $F_{\mathrm{ph}}(\omega)$.
This result is strong evidence for the importance of EPI in the pairing potential of HTSC cuprates. For instance, plenty of break junctions made from Bi2212 single crystals [4245] show that the peaks (and shoulders) in $-d^{2} I / d V^{2}$ (or dips-negative peaks in $d^{2} I / d V^{2}$ ) coincide with the peaks (and shoulders) in the phonon density of states $F_{\mathrm{ph}}(\omega)$ measured by neutron scattering - see Figure 21.

The tunnelling spectra in Bi-2212 break junctions [4245], which are shown in Figure 21 indicates that the spectral function $\alpha^{2} F(\omega)$ is independent of magnetic field, which is in contradiction with the theoretical prediction based on the SFI pairing mechanism where this function should be sensitive to magnetic field. The reported broadening of the peaks in $\alpha^{2} F(\omega)$ is partly due to the gapless spectrum of $d$ wave pairing in HTSC cuprates. Additionally, the tunnelling density of states $N_{T}(\omega)$ at very low $T$ and for $\omega>\Delta$ shows a pronounced gap structure. It was found that $2 \bar{\Delta} / T_{c}=$ $6.2-6.5$, where $T_{c}=74-85 \mathrm{~K}$ and $\bar{\Delta}$ is some average value of the gap. In order to obtain $\alpha^{2} F(\omega)$ the inverse procedure was used by assuming $s$-wave superconductivity and the effective Coulomb parameter $\mu^{*} \approx 0.1$ [42-45]. The obtained $\alpha^{2} F(\omega)$ gives large EPI coupling constant $\lambda_{e p} \approx 2.3$. Although this analysis [42-45] was done in terms of $s$-wave pairing, it mimics qualitatively the case of $d$-wave pairing, since one expects that $d$-wave pairing does not change significantly the global structure of $d^{2} I / d V^{2}$ at $\mathrm{eV}>\Delta$ albeit introducing a broadening in it-see the physical meaning in Appendix A. We point out that the results obtained in [42-45] were reproducible on more than 30 junctions. In that respect very important results on slightly overdoped Bi2212-GaAs and on Bi2212-Au planar tunnelling junctions are obtained in $[46,47]$ — see Figure 22.

These results show very similar features to those obtained in [42-45] on break junctions. It is worth mentioning that several groups [48-52] have obtained similar results for the shape of the spectral function $\alpha^{2} F(\omega)$ from the $I-V$ measurements on various HTSC cuprates-see the comparison in Figure 23. These facts leave no much doubts about the importance of the EPI in pairing mechanism of HTSC cuprates.

In that respect, the tunnelling measurements on slightly overdoped $\mathrm{Bi}_{2} \mathrm{Sr}_{2} \mathrm{CaCu}_{2} \mathrm{O}_{8}[46,47,53,54]$ give impressive results. The Eliashberg spectral function $\alpha^{2} F(\omega)$ of this compound was extracted from the measurements of $d^{2} I / d V^{2}$ and by solving the inverse problem-see Appendix A. The extracted $\alpha^{2} F(\omega)$ has several peaks in broad energy region up to $80 \mathrm{meV}$ as it is seen in Figures 22 and 23, which coincide rather well with the peaks in the phonon density of states $F_{\mathrm{ph}}(\omega)$-more precisely the generalized phonon density of states GPDS $(\omega)$ defined in Appendix A. In $[53,54]$ numerous peaks, from $P 1-P 13$, in $\alpha^{2} F(\omega)$ are discerned as shown in Figure 24, which correspond to various groups of phonon modes-laying in (and around) these peaks. Moreover, in $[46,47,53,54]$ the coupling constants for these modes are extracted as well as their contribution $\left(\Delta T_{c}\right)$ to $T_{c}$ as it is seen in Table 1 . Note that due to the nonlinearity of the problem the sum of $\left(\Delta T_{c}\right)_{i}, i=1,2, \ldots, 13$, due to various modes is not equal to $T_{c}$. 


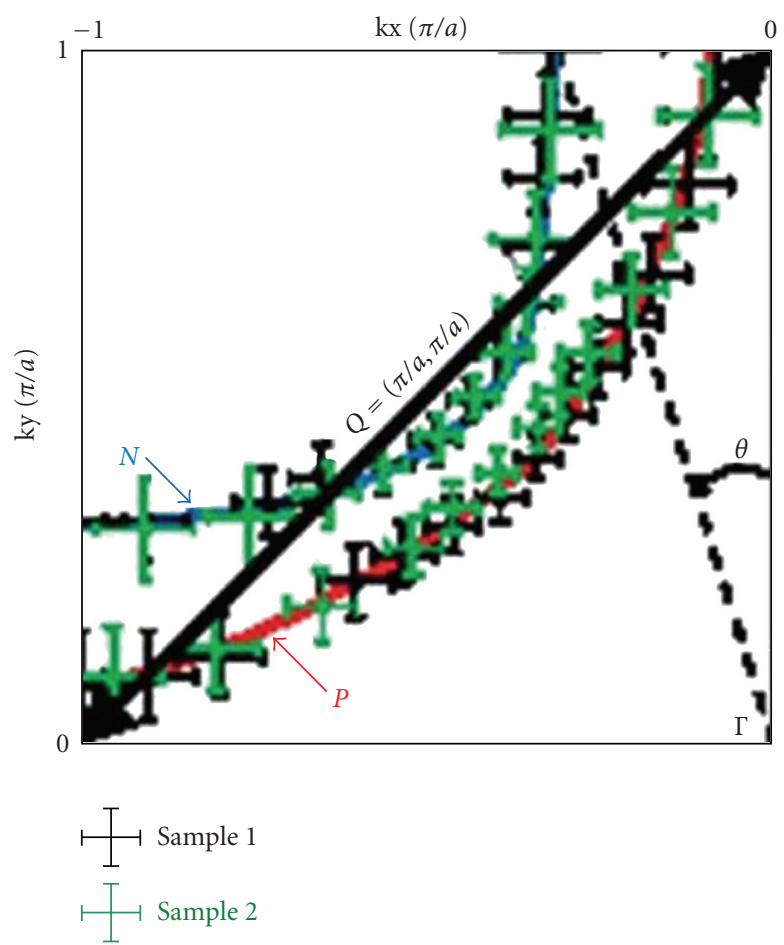

(a)

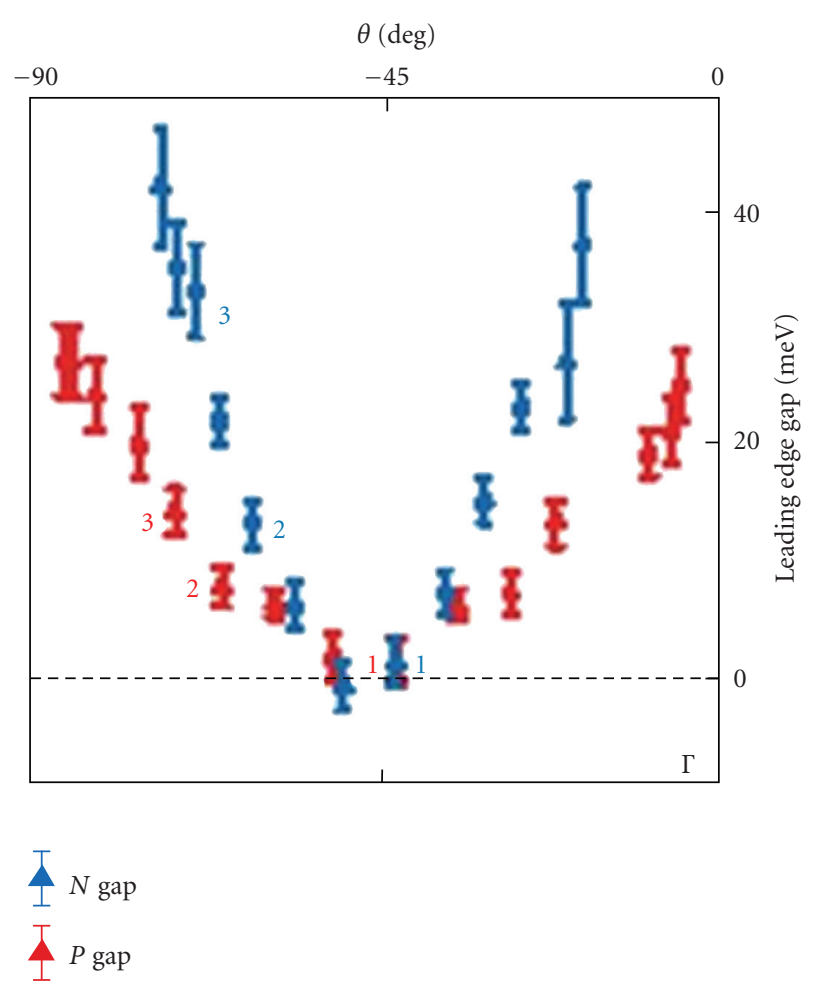

(b)

Figure 20: (a) Fermi surface (FS) contours from two samples of F0234. $N$-electron-like; $P$-hole-like. Bold arrow is $(\pi, \pi)$ scattering vector. Angle $\theta$ defines the horizontal axis in (b). (b) Leading gap edge along k-space angle from the two FS contours. From [152].

The next remarkable result is that the extracted EPI coupling constant is very large, that is, $\lambda_{e p}\left(=2 \int d \omega \alpha^{2} F(\omega) / \omega\right)=$ $\sum_{i} \lambda_{i} \approx 3.5$ - see Table 1 . It is obvious from Figure 24 and Table 1 that almost all phonon modes contribute to $\lambda_{e p}$ and $T_{c}$, which means that on the average each particular phonon mode is not too strongly coupled to electrons since $\lambda_{i}<$ $1.3, i=1,2, \ldots, 13$, thus keeping the lattice stable.

Let us discuss the content of Table 1 in more details where it is shown the strength of the EPI coupling and the relative contribution of different phononic modes to $T_{c}$. In Table 1 it is seen that lower-frequency modes from $P 1-P 3$, corresponding to $\mathrm{Cu}, \mathrm{Sr}$, and $\mathrm{Ca}$ vibrations, are rather strongly coupled to electrons (with $\lambda_{\kappa} \sim 1$ ) which give appreciable contributions to $T_{c}$. It is also seen in Table 1 that the coupling constants $\lambda_{i}$ of the high-energy phonons ( $P 9-P 13$ with $\omega \geq 70 \mathrm{meV}$ ) have $\lambda_{i} \ll 1$ and give moderate contribution to $T_{c}$-around $10 \%$. These results give solid evidence for the importance of the lowenergy modes related to the change of the Madelung energy in the ionic-metallic structure of HTSC cuprates- the idea advocated in [3-6] and discussed in Section 2. If confirmed in other HTSC families, these results are in favor of the moderate oxygen isotope effect in cuprates near the optimal doping since the oxygen modes are higher-energy modes and give smaller contribution to $T_{c}$. We stress that each peak $P 1-P 13$ in $\alpha^{2} F(\omega)$ corresponds to many modes. For a better understanding of the EPI coupling in these systems we show in Figure 25 the total and partial density of phononic states. It
TABLE 1: Phonon frequency $\omega$, EPI coupling constant $\lambda_{i}$ of the peaks $P 1-P 13$, and contribution $\Delta T_{c}$ to $T_{c}$ of each peak in $\alpha^{2} F(\omega)$ - as shown in Figure 24-obtained from the tunnelling conductance of $\mathrm{Bi}_{2} \mathrm{Sr}_{2} \mathrm{CaCu}_{2} \mathrm{O}_{8} . \Delta T_{c}$ is the decrease in $T_{c}$ when the peak in $\alpha^{2} F(\omega)$ is eliminated. From $[53,54]$.

\begin{tabular}{lccc}
\hline No. of peak & $\omega[\mathrm{meV}]$ & $\lambda_{i}$ & $\Delta T_{c}[\mathrm{~K}]$ \\
\hline$P 1$ & 14.3 & 1.26 & 7.4 \\
$P 2$ & 20.8 & 0.95 & 11.0 \\
$P 3$ & 31.7 & 0.48 & 10.5 \\
$P 4$ & 35.1 & 0.28 & 6.7 \\
$P 5$ & 39.4 & 0.24 & 7.0 \\
$P 6$ & 45.3 & 0.30 & 10.0 \\
$P 7$ & 58.3 & 0.15 & 6.5 \\
$P 8$ & 63.9 & 0.01 & 0.6 \\
$P 9$ & 69.9 & 0.07 & 3.6 \\
$P 10$ & 73.7 & 0.06 & 3.3 \\
$P 11$ & 77.3 & 0.01 & 0.8 \\
$P 12$ & 82.1 & 0.01 & 0.7 \\
$P 13$ & 87.1 & 0.03 & 1.8 \\
\hline
\end{tabular}

is seen that the low-energy phonons are due to the vibrations of the $\mathrm{Ca}, \mathrm{Sr}$, and $\mathrm{Cu}$ ions which correspond to the peaks $P 1-P 2$ in Figures 23 and 24. In order to obtain information on the structure of vibrations which are strongly involved in pairing, we show in Figures 26 and 27 the structure of 


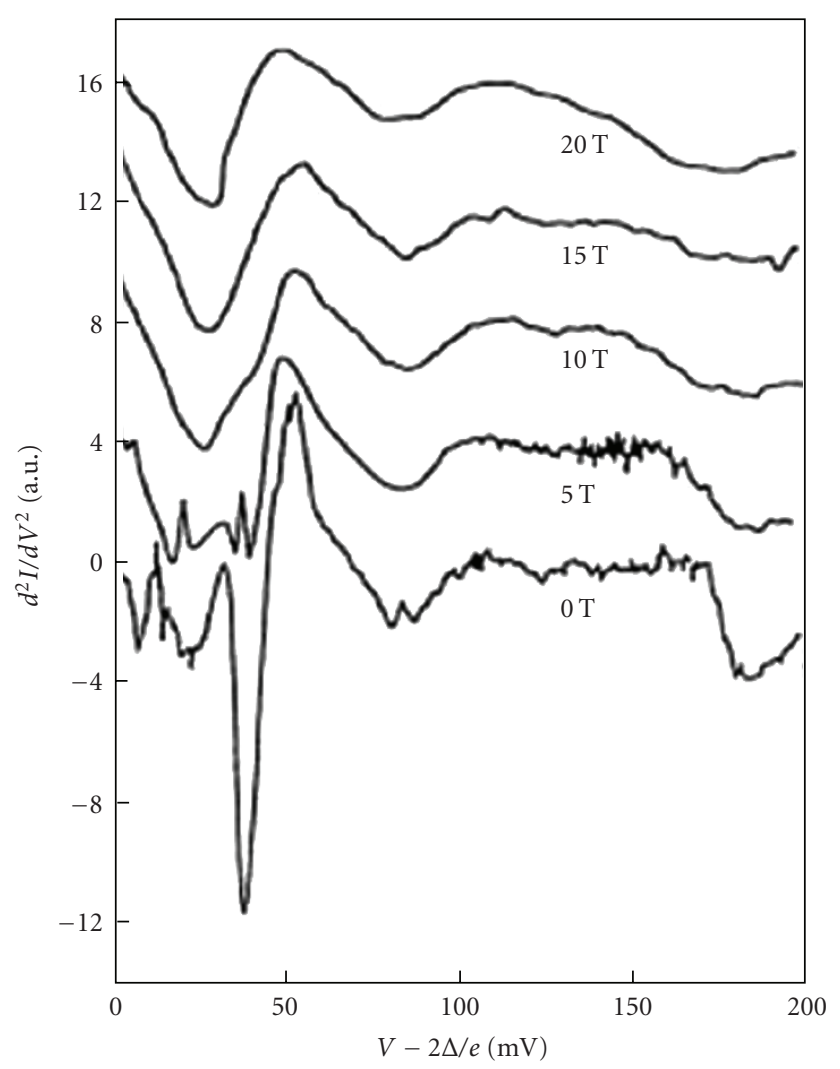

(a)

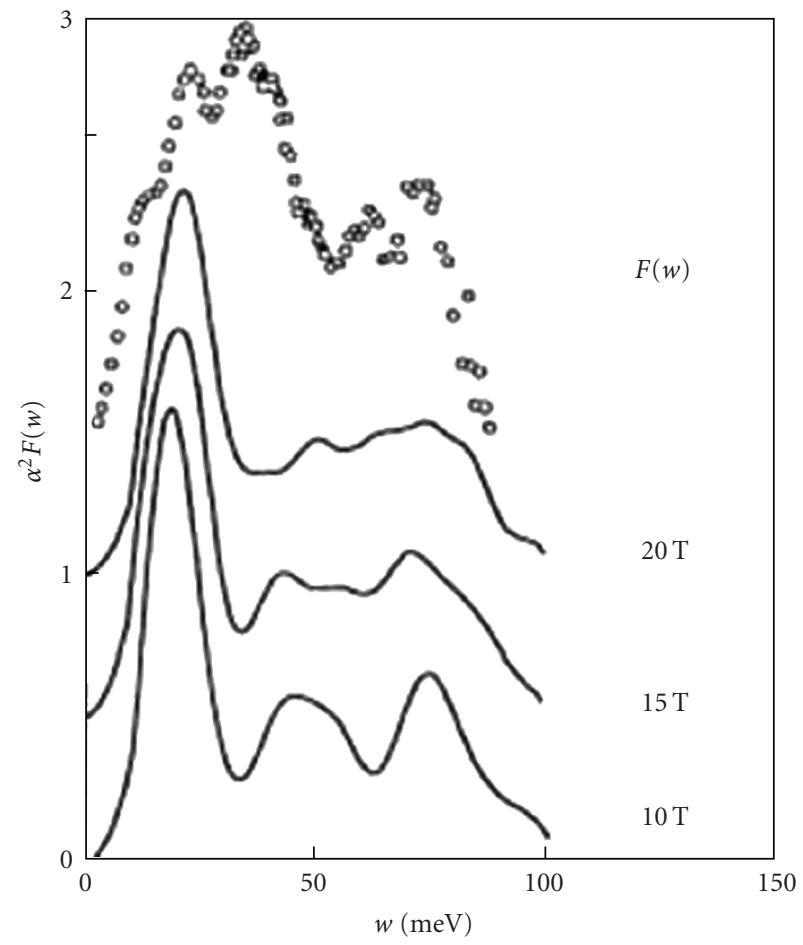

(b)

FIGURE 21: (a) Second derivative of $I(V)$ for a Bi2212 break junction in various magnetic fields (from 0-20 T). The structure of dips (minima) in $d^{2} I / d V^{2}$ can be compared with the phonon density of states $F(\omega)$; (b) the spectral functions $\alpha^{2} F(\omega)$ in various magnetic fields. From [4245].

these vibrations at special points in the Brillouin zone. It is seen in Figure 26 that the low-frequency phonons $P 1-P 2$ are dominated by $\mathrm{Cu}, \mathrm{Sr}$, Ca vibrations.

Further, based on Table 1 one concludes that the P3 modes are strongerly coupled to electrons than the $P 4$ ones, although the density of state for the $P 4$ modes is larger. The reason for such an anomalous behavior might be due to symmetries of the corresponding phonons as it is seen in Figure 27. Namely, to the $P 3$ peak contribute axial vibrations of $\mathrm{O}(1)$ in the $\mathrm{CuO}_{2}$ plane which are odd under inversion, while in the $P 4$ peak these modes are even. The in-plane modes of $\mathrm{Ca}$ and $\mathrm{O}(1)$ are present in $P 3$ which are in-phase and out-of-phase modes, while in $P 4$ they are all out-of-phase modes. For more information on other modes, $P 5-P 13$, see $[53,54]$. We stress that the Eliashberg equations based on the extracted $\alpha^{2} F(\omega)$ of the slightly overdoped $\mathrm{Bi}_{2} \mathrm{Sr}_{2} \mathrm{CaCu}_{2} \mathrm{O}_{8}$ with the ratio $\left(2 \Delta / T_{c}\right) \approx$ 6.5 describe rather well numerous optical, transport, and thermodynamic properties $[53,54]$. However, in underdoped systems with $\left(2 \Delta / T_{c}\right) \approx 10$, where the pseudogap phenomena are pronounced, there are serious disagreements between experiments and the Migdal-Eliashberg theory [53, 54]. We would like to stress that the contribution of the highfrequency modes (mostly the oxygen modes) to $\alpha^{2} F(\omega)$ may be underestimated in tunnelling measurements due to their sensitivity to the surface contamination and defects. Namely, the tunnelling current probes a superconductor to a depth of order of the quasiparticle mean-free path $l(\omega)=v_{F} \gamma^{-1}(\omega)$. Since the relaxation time $\gamma^{-1}(\omega)$ decreases with increasing $\omega$, the mean-free path can be rather small and the effects of the high-energy phonons are sensitive to the surface contamination.

Similar conclusion regarding the structure of the EPI spectral function $\alpha^{2} F(\omega)$ in HTSC cuprates comes out from tunnelling measurements on Andreev junctions (the BTK parameter $Z \ll 1$-low barrier) and Giaver junctions $(Z \gg$ 1-high barrier) in $\mathrm{La}_{2-x} \mathrm{Sr}_{x} \mathrm{CuO}_{4}$ and $Y B C O$ compounds [160], where the extracted $\alpha^{2} F(\omega)$ is in good accordance with the phonon density of states $F_{\mathrm{ph}}(\omega)$ - see Figure 28 .

Note that the BTK parameter $Z$ is related to the transmission and reflection coefficients for the normal metal $\left(1+Z^{2}\right)^{-1}$ and $Z^{2}\left(1+Z^{2}\right)^{-1}$, respectively.

Although most of the peaks in $\alpha^{2} F(\omega)$ of HTSC cuprates coincide with the peaks in the phonon density of states, it is legitimate to put the following question. Can the magnetic resonance in the superconducting state give significant contribution to $\alpha^{2} F(\omega)$ ? In that respect the inelastic magnetic neutron scattering measurements of the magnetic resonance as a function of doping [161] give that the resonance energy $E_{r}$ scales with $T_{c}$, that is, $E_{r}=(5-6) T_{c}$ as shown in Figure 29. 


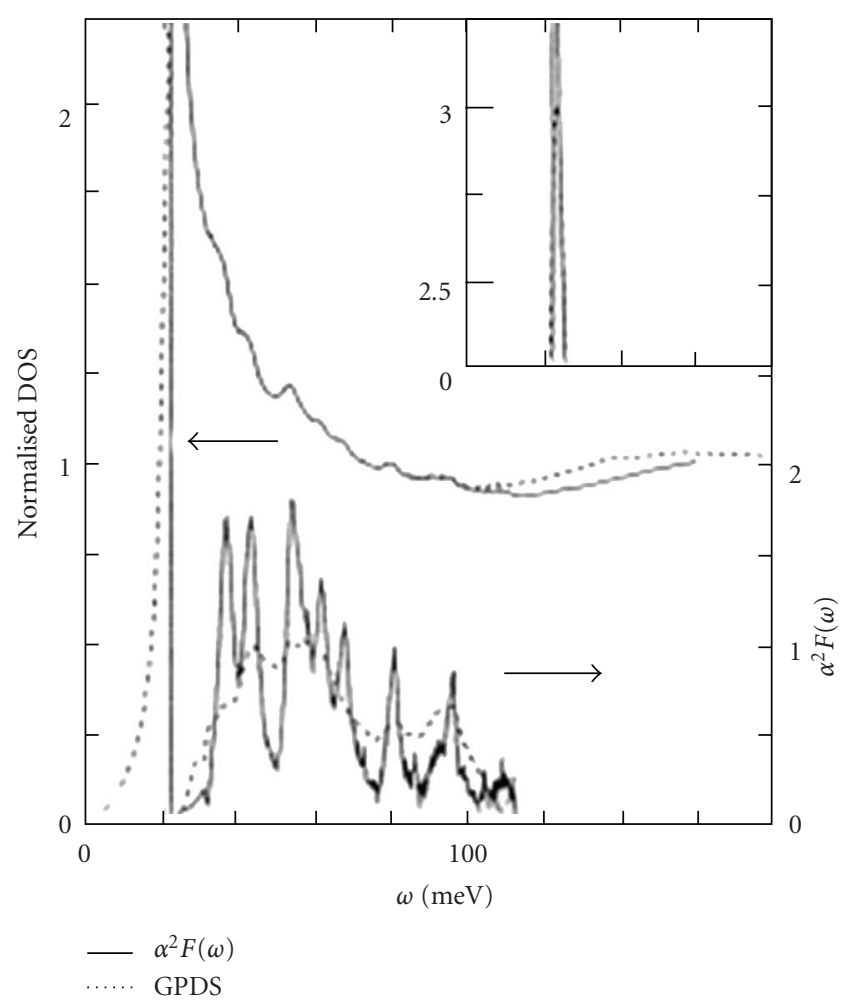

Figure 22: The spectral functions $\alpha^{2} F(\omega)$ and the calculated density of states at $0 \mathrm{~K}$ (upper solid line) obtained from the conductance measurements, the $\mathrm{Bi}(2212)-\mathrm{Au}$ planar junctions. GPDS—-generalized phonon density of states. From [46, 47].

This means that if one of the peaks in $\alpha^{2} F(\omega)$ is due to the magnetic resonance at $\omega=E_{r}$, then it must shift strongly with doping as it is observed in [161]. This is contrary to phonon peaks (energies) whose positions are practically doping independent. To this end, recent tunnelling experiments on Bi-2212 [55] show clear doping independence of $\alpha^{2} F(\omega)$ as it is seen in Figure 30. This remarkable result is an additional evidence in favor of EPI and against the SFI mechanism of pairing in HTSC cuprates which is based on the magnetic resonance peak in the superconducting state. In that respect the analysis in [162] of the tunneling spectra of the electron-doped cuprate $\operatorname{Pr}_{0.88} \mathrm{Ce}_{0.12} \mathrm{CuO}_{4}$ with $T_{c}=24 \mathrm{~K}$ shows the existence of the bosonic mode at $\omega_{B}=16 \mathrm{meV}$ which is significantly larger than the magneticresonance mode with $\omega_{r}=(10-11) \mathrm{meV}$. This result excludes the magnetic-resonance mode as an important factor which modifies superconductivity.

The presence of pronounced phononic structures (and the importance of EPI) in the $I(V)$ characteristics was quite recently demonstrated by the tunnelling measurements on the very good $\mathrm{La}_{1.85} \mathrm{Sr}_{0.15} \mathrm{CuO}_{4}$ films prepared by the molecular beam epitaxy on the [001]-symmetric $\mathrm{SrTiO}_{3}$ bicrystal substrates [56]. They give unique evidence for eleven peaks in the (negative) second derivative, that is, $-d^{2} I / d V^{2}$. Furthermore, these peaks coincide with the peaks in the intensities of the phonon Raman scattering data measured at $30 \mathrm{~K}$ in single crystals of LSCO with $20 \%$ of Sr [57]. These results are shown in Figure 31. In spite of the

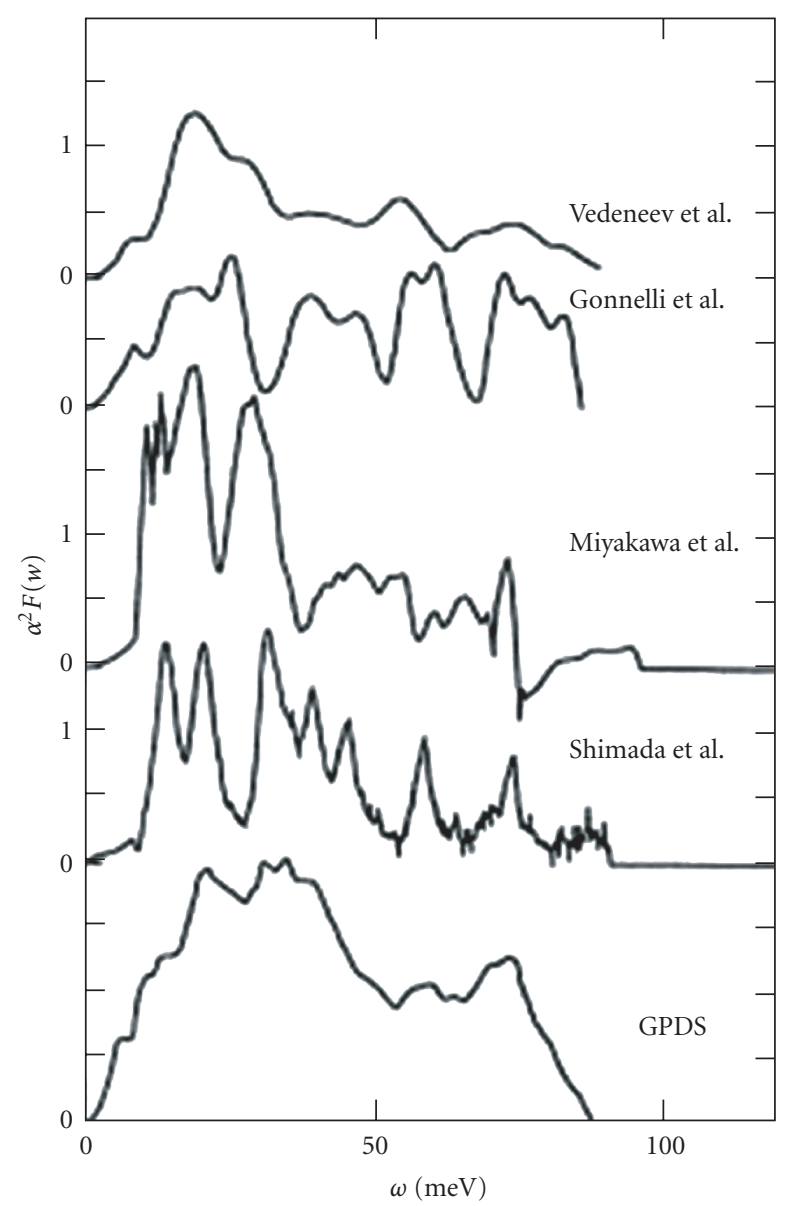

FIGURE 23: The spectral functions $\alpha^{2} F(\omega)$ from measurements of various groups: Vedeneev et al. [42-45], Gonnelli et al. [52], Miyakawa et al. [48, 49], and Shimada et al. [46, 47]. The generalized density of states GPDS for Bi2212 is at the bottom. From $[46,47]$.

lack of a quantitative analysis of the data in the framework of the Eliashberg equations, the results in [56] are important evidence that phonons are relevant pairing bosons in HTSC cuprates.

It is interesting that in the $c$-axis vacuum tunnelling STM measurements [163] the fine structure in $d^{2} I / d V^{2}$ at $\mathrm{eV}>\Delta$ was not seen below $T_{c}$, while the pseudogap structure is observed at temperatures near and above $T_{c}$. This result could mean that the STM tunnelling is likely dominated by the nontrivial structure of the tunnelling matrix element (along the $c$-axis), which is derived from the band-structure calculations [164]. However, recent STM experiments on Bi2212 [61-63] give information on the possible nature of the bosonic mode which couples with electrons. In [61-63] the local conductance $d I / d V(\mathbf{r}, E)$ is measured where it is found that $d^{2} I / d V^{2}(\mathbf{r}, E)$ has peak at $E(\mathbf{r})=\Delta(\mathbf{r})+\Omega(\mathbf{r})$ where $d I / d V(\mathbf{r}, E)$ has the maximal slope-see Figure 32(a).

It turns out that the corresponding average phonon energy $\bar{\Omega}$ depends on the oxygen mass, that is, $\bar{\Omega} \sim M_{\mathrm{O}}^{-1 / 2}$, with $\bar{\Omega}_{16}=52 \mathrm{meV}$ and $\bar{\Omega}_{18} \approx 48 \mathrm{meV}$-as it is seen in Figure 32(b). This result is interpreted in [61-63] as an evidence that the oxygen phonons are strongly involved in 


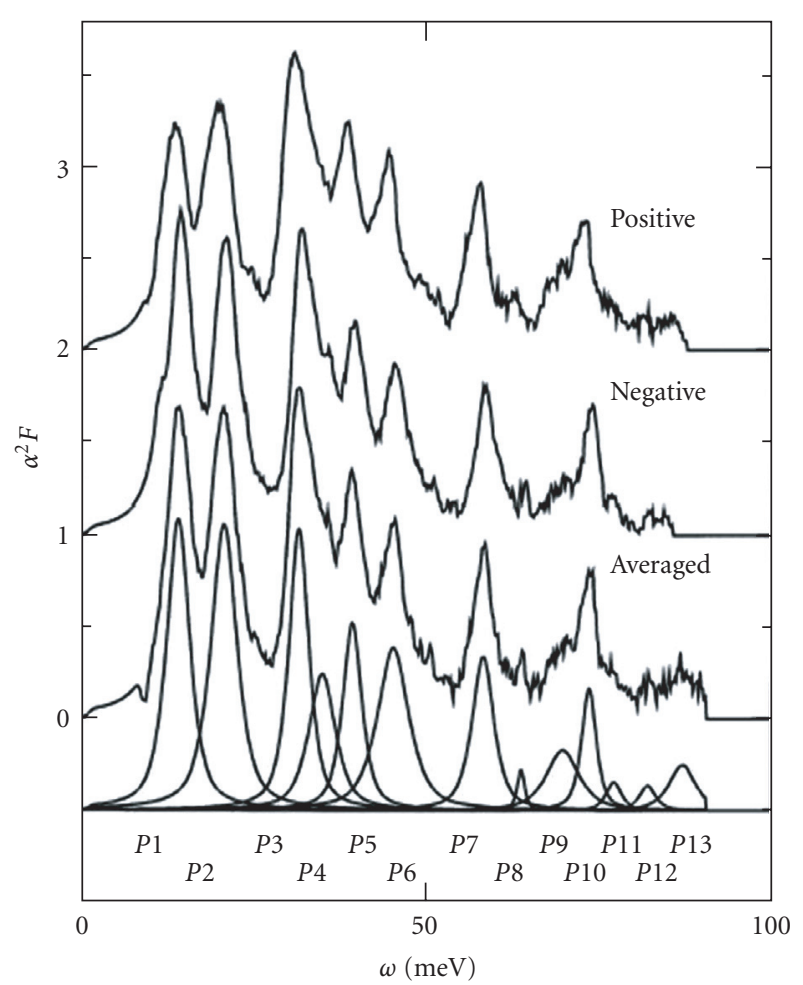

Figure 24: The spectral functions $\alpha^{2} F(\omega)$ from the tunnelling conductance of $\mathrm{Bi}_{2} \mathrm{Sr}_{2} \mathrm{CaCu}_{2} \mathrm{O}_{8}$ for the positive and the negative bias voltages, and the averaged one $[46,47]$. The averaged one is divided into 13 components. The origin of the ordinate is $2,1,0$, and -0.5 from the top down. From $[46,47,53,54]$.

the quasiparticle scattering. A possible explanation is put forward in [61-63] by assuming that this isotope effect is due to the $B_{1 g}$ phonon which interacts with the antinodal quasiparticles. However, this result requires a reanalysis since the energy of the bosonic mode in fact coincides with the dip and not with the peak of $d^{2} I / d V^{2}(\mathbf{r}, E)$ - as reported in [61-63].

The important message of numerous tunnelling experiments in HTSC cuprates near and at the optimal doping is that there is strong evidence for the importance of EPI in the quasiparticle scattering and that no particular phonon mode can be singled out in the spectral function $\alpha^{2} F(\omega)$ as being the only one which dominates in pairing mechanism. This important result means that the high $T_{c}$ is not attributable to a particular phonon mode in the EPI mechanism but all phonon modes contribute to $\lambda_{e p}$. Having in mind that the phonon spectrum in HTSC cuprates is very broad (up to $80 \mathrm{meV}$ ), then the large EPI constant $\left(\lambda_{e p} \gtrsim 2\right)$ obtained in the tunnelling experiments is not surprising at all. Note that similar conclusion holds for some other oxide superconductors such as $\mathrm{Ba}_{9.6} \mathrm{~K}_{0.4} \mathrm{BiO}_{3}$ with $T_{c}=30 \mathrm{~K}$ where the peaks in the bosonic spectral function/extracted from tunnelling measurements coincide with the peaks in the phononic density of states [165-167].

1.3.5. Phonon Spectra and EPI. Although experiments related to phonon spectra and their renormalization by $E P I$, such as inelastic neutron, inelastic X-ray, and Raman

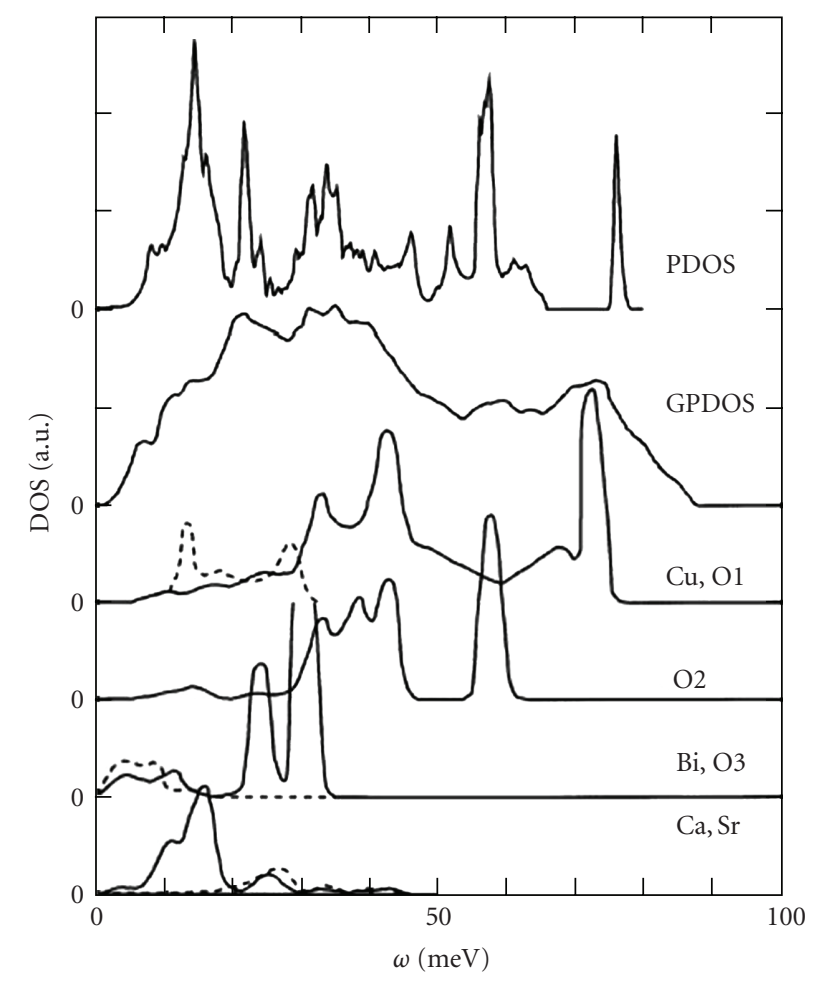

Figure 25: The phonon density of states $F(\omega)$ (PDOS) of $\mathrm{Bi}_{2} \mathrm{Sr}_{2} \mathrm{CaCu}_{2} \mathrm{O}_{8}$ compared with the generalized density of states (GPDOS) [159]. Atomic vibrations: $\mathrm{O} 1-\mathrm{O}$ in the $\mathrm{CuO}_{2}$ plane; $\mathrm{O} 2-$ apical O; O3-O in the $\mathrm{BiO}$ plane. From $[46,47]$.

scattering, do not give the spectral function $\alpha^{2} F(\omega)$, they nevertheless can give useful, but indirect, information on the strength of EPI for some particular phonons. We stress in advance that the interpretation of the experimental results in HTSC cuprates by the theory of EPI for weakly correlated electrons is inadequate since in strongly correlated systems, such as HTSC cuprates, the phonon renormalization due to EPI is different than in weakly correlated metals [168]. Since these questions are reviewed in [168], we will briefly enumerate the main points: (1) in strongly correlated systems the EPI coupling for a number of phononic modes can be significantly larger than the LDA-DFT and Hartree-Fock methods predict. This is due to many-body effects not contained in LDA-DFT $[168,169]$. The lack of the LDA-DFT calculations in obtaining phonon line-widths is clearly demonstrated, for instance, in experiments on $\mathrm{L}_{2-x} \mathrm{Sr}_{x} \mathrm{CuO}_{4}$ - see review in [170] and references therein, where the bond-stretching phonons at $\mathbf{q}=(0.3,0,0)$ are softer and much broader than the LDA-DFT calculations predict. (Note the wave vector $\mathbf{q}$ is in units $(2 \pi / a, 2 \pi / b, 2 \pi / c)$-for instance, in these units $(\pi, \pi)$ corresponds to $(0.5,0.5)$.) (2) The calculation of phonon spectra is in principle very difficult problem since besides the complexity of structural properties in a given material one should take into account appropriately the long-range Coulomb interaction of electrons as well as strong short-range repulsion. Our intention is not to discuss this complexity here-for that see, for instance, [69] — but 

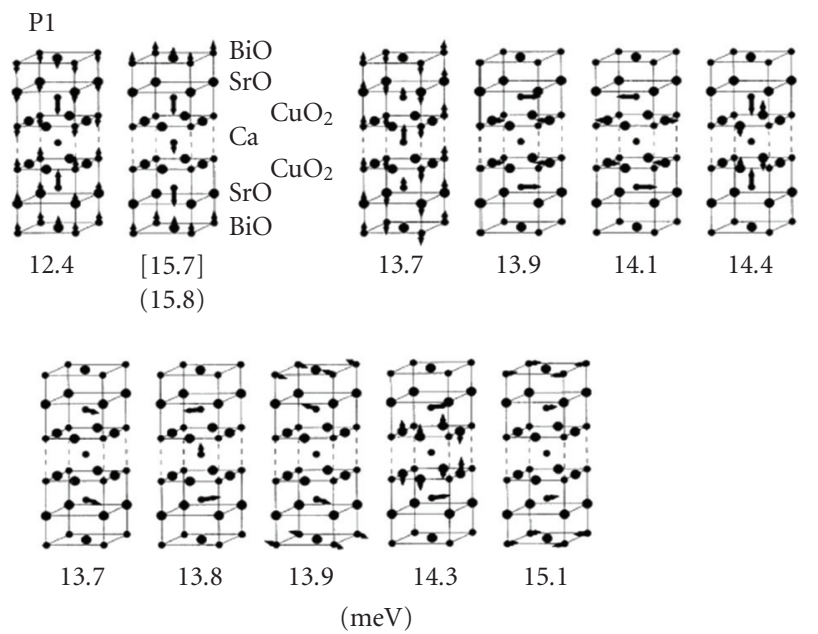

(a)

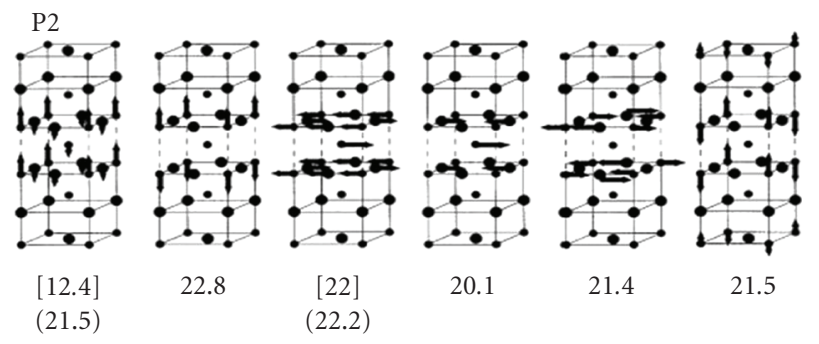

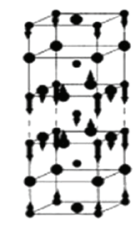

21.7

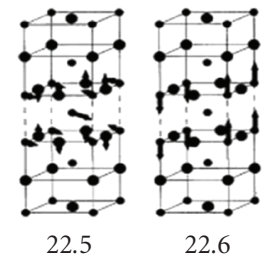

$(\mathrm{meV})$
Figure 26: Atomic polarization vectors and their frequencies (in $\mathrm{meV}$ ) at special points in the Brillouin zone. The larger closed circles in the lattice are $\mathrm{O}$-ions. $\Gamma-X$ is along the $\mathrm{Cu}-\mathrm{O}-\mathrm{Cu}$ direction. Arrows indicate displacements. The modes in square and round brackets are the transverse and longitudinal optical modes, respectively. (a) Modes of the P1 peak. (b) Modes of the P2 peak. From $[46,47,53,54]$.

we only stress some important points which will help to understand problems with which is confronted the theory of phonons in cuprates.

The phonon Green's function $D(\mathbf{q}, \omega)$ depends on the phonon self-energy $\Pi(\mathbf{q}, \omega)$ which takes into account all the enumerated properties (note that $D^{-1}(\mathbf{q}, \omega)=D_{0}^{-1}(\mathbf{q}, \omega)-$ $\Pi(\mathbf{q}, \omega))$. In cases when the EPI coupling constant $g_{e p}\left(\mathbf{k}, \mathbf{k}^{\prime}\right)$ is a function on the transfer momentum $\mathbf{q}=\mathbf{k}-\mathbf{k}^{\prime}$ only, then $\Pi(q)\left(q=\left(\mathbf{q}, i \omega_{n}\right)\right)$ depends on the quasiparticle charge susceptibility $\chi_{c}(q)=P(q) / \varepsilon_{e}(q)$ :

$$
\Pi(q)=\left|g_{e p}(\mathbf{q})\right|^{2} \chi_{c}(q),
$$
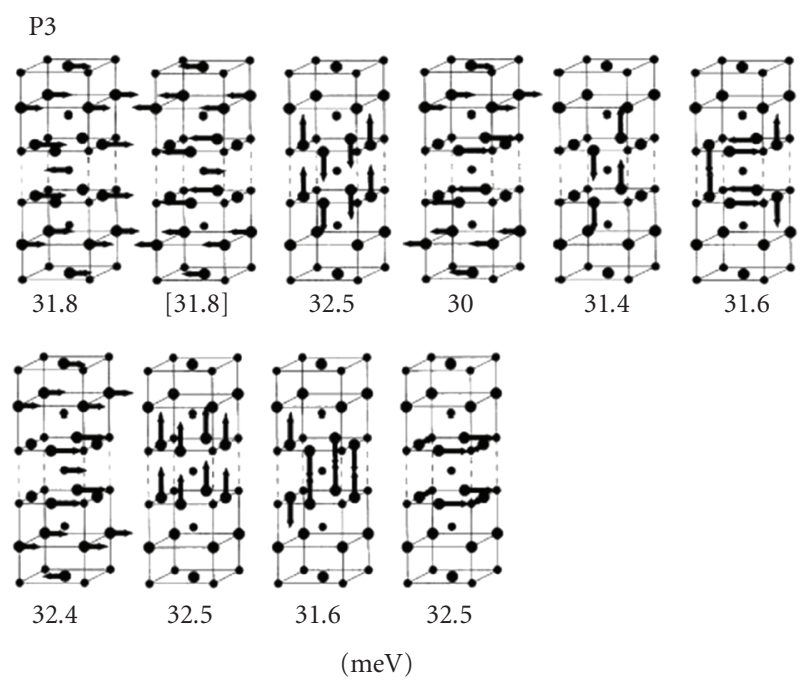

(a)

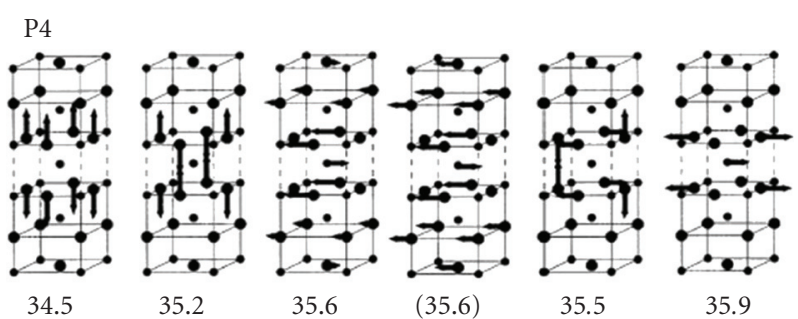

35.2

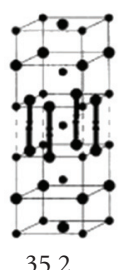

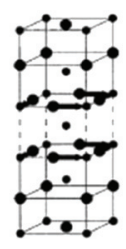

35.2 (b)

FIgURE 27: Atomic polarization vectors and their frequencies (in $\mathrm{meV}$ ) at special points in the Brillouin zone. The larger closed circles in the lattice are $\mathrm{O}$-ions. $\Gamma-X$ is along the $\mathrm{Cu}-\mathrm{O}-\mathrm{Cu}$ direction. Arrows indicate displacements. The modes in square and round brackets are the transverse and longitudinal optical modes, respectively. (a) Modes of the P3 peak. (b) Modes of the P4 peak. From $[46,47,53,54]$.

and $P(q)$ is the irreducible electronic polarization given by

$$
P(q)=-\sum_{p} G(p+q) \Gamma_{c}(p, q) G(p) .
$$

The screening due to the long-range Coulomb interaction is contained in the electronic dielectric function $\varepsilon_{e}(q)$ while the "screening" due to (strong) correlations is described by the charge vertex function $\Gamma_{c}(p, q)$. Due to complexity of the physics of strong correlations the phonon dynamics was studied in the $t-J$ model but without the long-range Coulomb interaction $[168,169,171]$, in which case one has $\varepsilon_{e}=1$ and $\chi_{c}(q)=P(q)$. However, in studying the phonon 


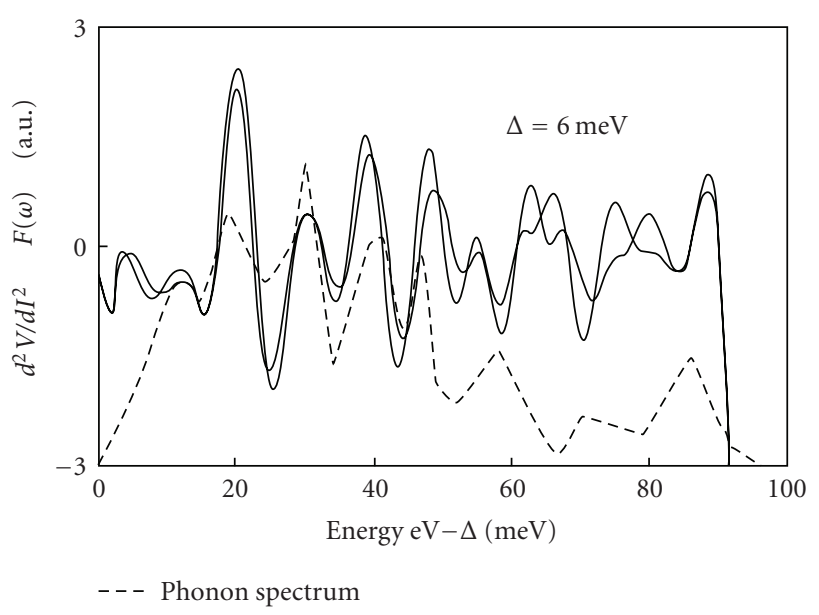

(a)

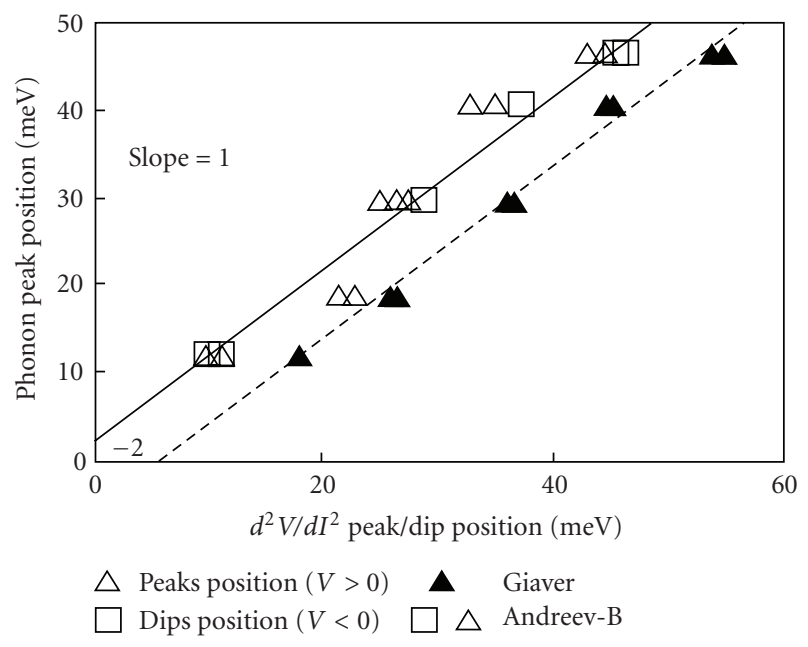

(b)

Figure 28: (a) $d^{2} I / d V^{2}$ of a Giaver-like contact in $\mathrm{La}_{2-x} \mathrm{Sr}_{x} \mathrm{CuO}_{4}-$ note the large structure below $50 \mathrm{meV}$; (b) $d^{2} I / d V^{2}$ of an Andreevand Giaver-like contact compared to the peaks in the phonon density of states. From [160].

spectra in HTSC cuprates it is believed that this deficiency might be partly compensated by choosing the bare phonon frequency $\omega_{0}(\mathbf{q})$ (contained in $D_{0}^{-1}(\mathbf{q}, \omega)$ ) to correspond to the undoped compounds $[168,171]$. It is a matter of future investigations to incorporate all relevant interactions in order to obtain a fully microscopic and reliable theory of phonons in cuprates. Additionally, the electron-phonon interaction (with the bare coupling constant $g_{e p}(\mathbf{q})$ ) is dominated by the change of the energy of the Zhang-Rice singlet-see more in Section 2.3-and (41) for $\Pi(q)$ is adequate one $[6,168,169]$. Since the charge fluctuations in HTSC cuprates are strongly suppressed (no doubly occupancy of the $\mathrm{Cu} 3 \mathrm{~d}^{9}$ state) due to strong correlations, and since the suppressed value of $\chi_{c}(q)$ cannot be obtained by the band-structure calculations, this means that $L D A-D F T$ underestimates the EPI coupling constant significantly. In the following we discuss this important result briefly.

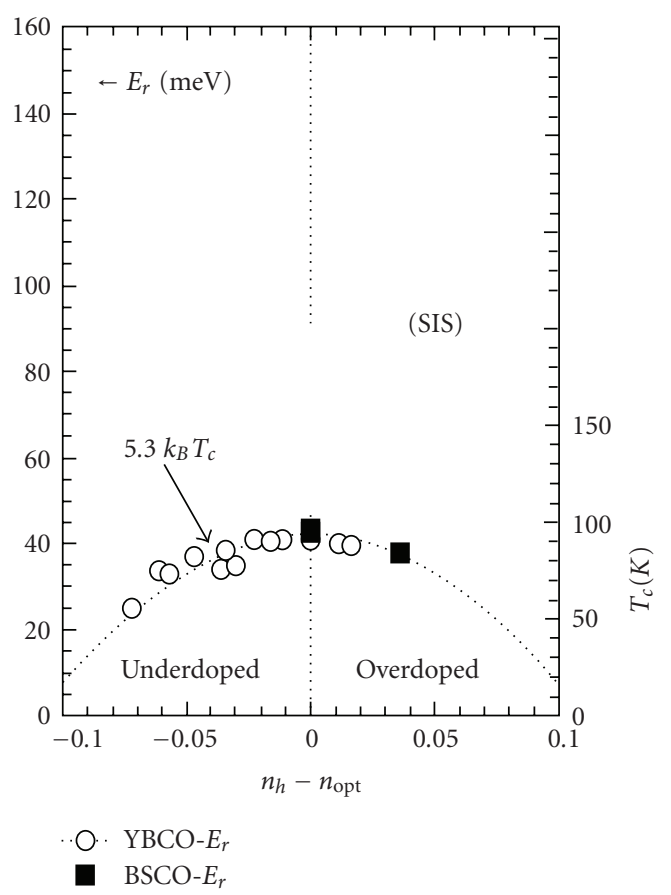

FIGURE 29: Doping dependence of the energy $E_{r}$ of the magnetic resonance peak at $\mathbf{Q}=(\pi, \pi)$ in YBCO and Bi2212 measured at low temperatures by inelastic neutron scattering. From [161].

(1) Inelastic Neutron and X-Ray Scattering-The Phonon Softening and the Line-Width due to EPI. The appreciable softening and broadening of numerous phonon modes has been observed in the normal state of HTSC cuprates, thus giving evidence for pronounced EPI effects and for inadequacy of the LDA-DFT calculations in treating strong correlations and suppression of the charge susceptibility $[6,10,11,168,171]$. There are several relevant reviews on this subject $[10,11,168$, $170,172]$ and here we discuss briefly two important examples which demonstrate the inefficiency of the LDA-DFT-band structure calculations to treat quantitatively EPI in HTSC cuprates. For instance, the $\mathrm{Cu}-\mathrm{O}$ bond-stretching phonon mode shows a substantial softening at $\mathbf{q}_{\mathrm{hb}}=(0.3,0,0)$ by doping of $\mathrm{La}_{1.85} \mathrm{Sr}_{0.15} \mathrm{CuO}_{4}$ and $\mathrm{YBa}_{2} \mathrm{Cu}_{3} \mathrm{O}_{7}[170,172]-$ called the half-breathing phonon, and a large broadening by $5 \mathrm{meV}$ at $15 \%$ doping [173-175] as it is seen in Figure 33. While the softening can be partly described by the LDADFT method [176], the latter theory predicts an order of magnitude smaller broadening than the experimental one. This failure of LDA-DFT is due to the incorrect treatment of the effects of strong correlations on the charge susceptibility $\chi_{c}(q)$ and due to the absence of many-body effects which can increase the coupling constant $g_{e p}(\mathbf{q})$-see more in Section 2. The neutron scattering measurements in $\mathrm{La}_{1.85} \mathrm{Sr}_{0.15} \mathrm{CuO}_{4}$ give evidence for large $(30 \%)$ softening of the $\mathrm{O}_{Z}^{Z}$ with $\Lambda_{1}$ symmetry with the energy $\omega \approx 60 \mathrm{meV}$, which is theoretically predicted in [177], and for the large line-width about $17 \mathrm{meV}$ which also suggests strong EPI. These apex modes are favorable for $d$-wave pairing since their coupling constants are peaked at small momentum $q[10,11]$. Having in mind the above results, then it is not surprising that 


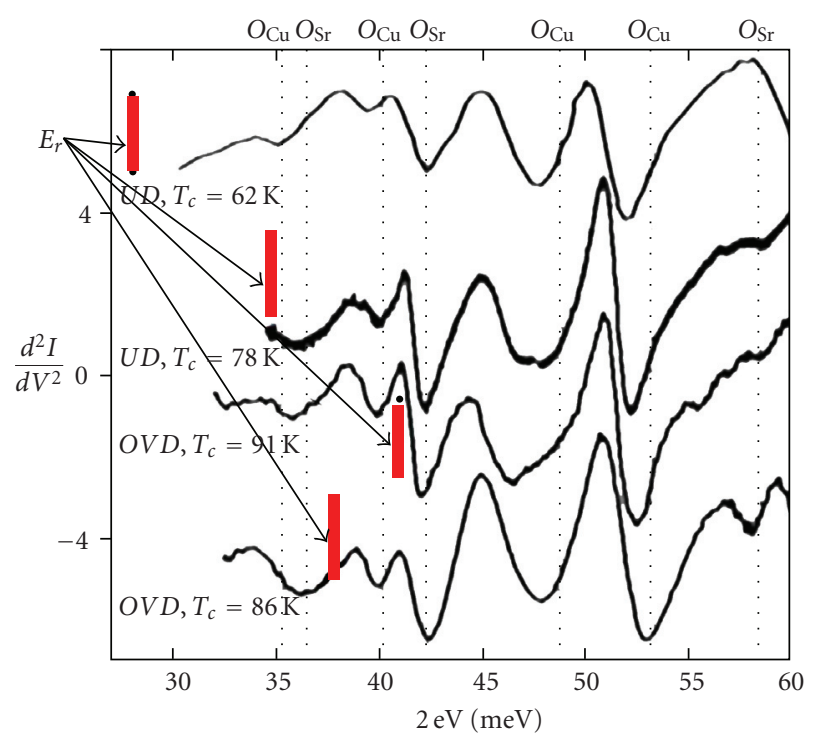

Figure 30: Second derivative of $I(V)$ for Bi2212 tunnelling junctions for various doping: UD—underdoped; OD—optimally doped; OVD-overdoped system. The structure of minima in $d^{2} I / d V^{2}$ can be compared with the phonon density of states $F(\omega)$. The full and vertical lines mark the positions of the magnetic resonance energy $E_{r} \approx 5.4 T_{c}$ for various doping taken from Figure 29. Red tiny arrows mark positions of the magnetic resonance $E_{r}$ in various doped systems. Dotted vertical lines mark various phonon modes. From [55].

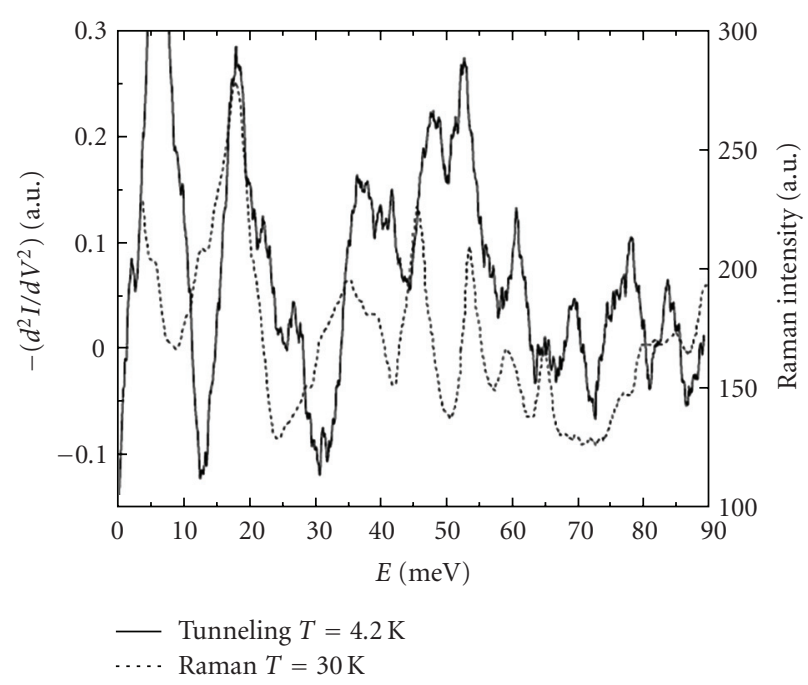

Figure 31: Second derivative data $d^{2} I(V) / d V^{2}$ of the tunnelling spectra on thin films of $\mathrm{La}_{1.85} \mathrm{Sr}_{0.15} \mathrm{CuO}_{4}$ are shown along with phonon Raman scattering data on single crystals of LSCO with $20 \%$ Sr. The polarization of the incident and scattered light in the Raman spectra is parallel to the $\mathrm{CuO}_{2}$ planes. From [56].

the recent calculations of the EPI coupling constant $\lambda_{e p}$ in the framework of LDA-DFT give very small EPI coupling constant $\lambda_{e p} \approx 0.3[28,29]$. The critical analysis of the LDADFT results in HTSC cuprates is done in [6] and additionally argued in $[10,11,178]$ by pointing their disagreement with the inelastic neutron and X-ray scattering measurements-as it is shown in Figure 33.

In Section 2 we will discuss some theoretical approaches related to EPI in strongly correlated systems but without discussing the phonon renormalization. The latter problem was studied in more details in the review articles in [10, $11,170]$. Here, we point out only three (for our purposes) relevant results. First, there is an appreciable difference in the phonon renormalization in strongly and weakly correlated systems. Namely, the change of the phonon frequencies in the presence of the conduction electrons is proportional to the squared coupling constant $\left|g_{\mathbf{q}}\right|$ and charge susceptibility $\chi_{c}$, that is, $\delta \omega(\mathbf{q}) \sim\left|g_{e p}(\mathbf{q})\right|^{2} \operatorname{Re} \chi_{c}$, while the line-width is given by $\Gamma_{\omega(\mathbf{q})} \sim\left|g_{e p}(\mathbf{q})\right|^{2}\left|\operatorname{Im} \chi_{c}\right|$. All these quantities can be calculated in LDA-DFT and as we discussed above, where for some modes one obtains that $\Gamma_{\omega(\mathbf{q})}^{(\mathrm{LDA})} \ll \Gamma_{\omega(\mathbf{q})}^{(\mathrm{exp})}$. However, it turns out that in strongly correlated systems doped by holes (with the concentration $\delta \ll 1$ ) the charge fluctuations are suppressed in which case the following sum rule holds $[10,11,171]$ :

$$
\frac{1}{\pi N} \sum_{\mathbf{q} \neq 0} \int_{-\infty}^{\infty} d \omega\left|\operatorname{Im} \chi_{c}(\mathbf{q}, \omega)\right|=2 \delta(1-\delta) N
$$

while in the LDA-DFT method one has

$$
\frac{1}{\pi N} \sum_{\mathbf{q} \neq 0} \int_{-\infty}^{\infty} d \omega\left|\operatorname{Im} \chi_{c}(\mathbf{q}, \omega)\right|^{(\mathrm{LDA})}=(1-\delta) N .
$$

The inequality $\Gamma_{\omega(\mathbf{q})}^{(\mathrm{LDA})} \ll \Gamma_{\omega(\mathbf{q})}^{(\exp )}$ (for some phonon modes) together with (43)-(44) means that for low doping $\delta \ll 1$ the LDA calculations strongly underestimate the EPI coupling constant in the large portion of the Brillouin zone, that is, one has $\left|g_{e p}^{(\mathrm{LDA})}(\mathbf{q})\right| \ll\left|g_{e p}^{(\exp )}(\mathbf{q})\right|$. The large softening and the large line-width of the half-breathing mode at $q=(0.5,0)$, but very moderate effects for the breathing mode at $q=(0.5,0.5)$, are explained in the framework of the one slave-boson (SB) theory (for $U=\infty$ ) in [171], where $\chi_{c}(\mathbf{q}, \omega)$ (i.e., $\left.\Gamma_{c}(p, q)=\Gamma_{c}(\mathbf{p}, q)\right)$ is calculated in leading $\mathrm{O}(1 / N)$ order. We stress that there is another method for studying strong correlations-the $X$-method-where the controllable $1 / N$ expansion is performed in terms of the Hubbard operators and where the charge vertex $\Gamma_{c}(\mathbf{p}, q)$ is calculated $[6,78-80,130,179,180]$. It turns out that in the adiabatic limit $(\omega=0)$ the vertex functions $\Gamma_{c}\left(\mathbf{p}_{F}, \mathbf{q}\right)$ in these two methods have important differences. For instance, $\Gamma_{c}^{(X)}\left(\mathbf{p}_{F}, q\right)$ (in the X-method) is peaked at $\mathbf{q}=0$-the so called forward scattering peak (FSP)—while $\Gamma_{c}^{(\mathrm{SB})}\left(\mathbf{p}_{F}, \mathbf{q}\right)$ has maximum at finite $|\mathbf{q}| \neq 0[181]$ - see Section 2.3.5. The enumerated properties of $\Gamma_{c}^{(X)}\left(\mathbf{p}_{F}, q\right)$ are confirmed by the numerical Monte Carlo calculations in the finite- $U$ Hubbard model [182], where it is found that FSP exists for all $U$, but it is especially pronounced in the limit $U \gg t$. These results are also confirmed in [183] where the calculations are performed in the four-slave-boson technique-see more in Section 2.3.5. Having in mind this difference it would be useful to have calculations of $\chi_{c}(\mathbf{q}, \omega)$ in the framework of the X-method which are unfortunately not done yet. Second, 


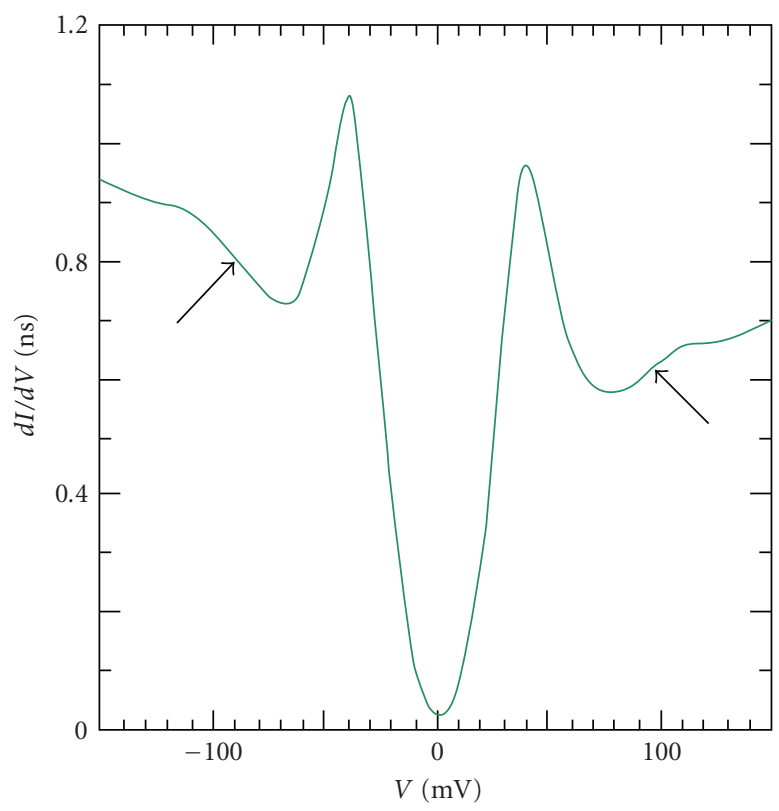

(a)

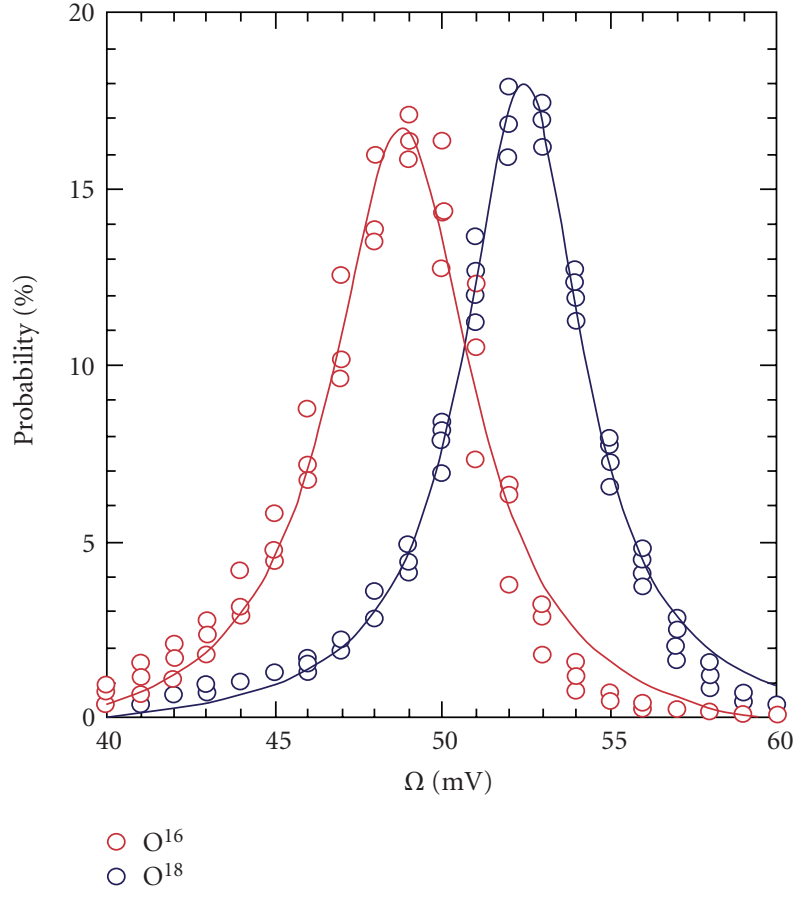

(b)

Figure 32: (a) Typical conductance $d I / d V(\mathbf{r}, E)$. The ubiquitous features at $\mathrm{eV}>\Delta$ (gap) with maximal slopes, which give peaks in $d^{2} I / d V^{2}(\mathbf{r}, E)$, are indicated by arrows. (b) The histograms of all values of $\Omega(\mathbf{r})$ for samples with $\mathrm{O}^{16}$-right curve and with $\mathrm{O}^{18}$-left curve. From [61-63].

the many-body theory gives that for coupling to some modes the coupling constant $\left|g_{e p}(\mathbf{q})\right|$ in HTSC cuprates can be significantly larger than the LDA-DFT calculations predict $[10,11]$, which is due to some many-body effects not present in the latter [169]. In Section 2 it will be argued that for some phonon modes one has $\left|g_{e p}(\mathbf{q})\right|^{2} \gg\left|g_{e p}^{(\mathrm{LDA})}(\mathbf{q})\right|^{2}$. For instance, for the half-breathing mode, one has $\left|g_{e p}(\mathbf{q})\right|^{2} \approx$ $3\left|g_{e p}^{(\mathrm{LDA})}(\mathbf{q})\right|^{2}[10,11,169]$ —see Section 2. These two results point to an inadequacy of LDA-DFT in calculations of EPI effects in HTSC cuprates. Third, the phonon self-energy $(\Pi(q))$ and quasiparticle self-energy $\Sigma(k)$ are differently renormalized by strong correlations $[6,10,11,78-80,130$, $179,180]$, which is the reason that $\Pi(q)$ is much more suppressed than $\Sigma(k)$-see Section 2. The effects of the charge vertex on $\Pi(q)$ and $\Sigma(k)$ are differently manifested. Namely, the vertex function enters quadratically in $\Sigma(k)$ and the presence of the forward scattering peak in the charge vertex strongly affects the EPI coupling constant $g_{e p}(\mathbf{q})$ in $\Sigma(k)$ :

$$
\Sigma(k)=-\sum_{q}\left|g_{e p}(\mathbf{q}) \gamma_{c}(\mathbf{k}, q)\right|^{2} D(q) g(k+q),
$$

where $g(k)(\equiv G(k) / Q)$ is the quasiparticle Green's function, $\gamma_{c}(\mathbf{k}, q)=\Gamma_{c}(\mathbf{k}, q) / Q$ is the quasiparticle vertex, and $Q(\sim \delta)$ is the Hubbard quasiparticle spectral weight—see Section 2.3. In the adiabatic limit $|\mathbf{q}|>q_{\omega}=\omega_{\mathrm{ph}} / \nu_{F}$ one has $\gamma_{c}(\mathbf{k}, q) \approx$ $\gamma_{c}(\mathbf{k}, \mathbf{q})$ and for $q \gg q_{c}(\approx \delta \cdot \pi / a)$ the charge vertex is strongly suppressed $\left(\gamma_{c}(\mathbf{k}, \mathbf{q}) \ll 1\right)$ making the effective EPI coupling (which also enters the pairing potential) small at large (transfer) momenta q. This has strong repercussion on the pairing due to EPI since for small doping it makes the $d$-wave pairing coupling constant to be of the order of the $s$-one $\left(\lambda_{d} \approx \lambda_{s}\right)$. Then in the presence of the residual Coulomb interaction EPI gives rise to $d$-wave pairing. On the other side the charge vertex $\Gamma_{c}(\mathbf{k}, q)$ enters $\Pi(q)$ linearly and it is additionally integrated over the quasiparticle momentum $\mathbf{k}$-see (42). Therefore, one expects that the effects of the forward scattering peak on $\Pi(q)$ are less pronounced than on $\Sigma(k)$. Nevertheless, the peak of $\Gamma_{c}(\mathbf{k}, \mathbf{q})$ at $\mathbf{q}=0$ may be (partly) responsible that the maximal experimental softening and broadening of the stretching (half-breathing) mode in $\mathrm{La}_{1.85} \mathrm{Sr}_{0.15} \mathrm{CuO}_{4}$ and $\mathrm{YBa}_{2} \mathrm{Cu}_{3} \mathrm{O}_{7}$ is at $\mathbf{q}_{\mathrm{hb}}^{(\exp )}=(0.3,0,0)$ [170] and not at $\mathbf{q}_{\mathrm{hb}}=(0.5,0)$ for which $g_{e p}\left(\mathbf{q}_{\mathrm{hb}}\right)$ reaches maximum. This means that the charge vertex function pushes the maximum of the renormalized EPI coupling constant to smaller momenta q. It would be very interesting to have calculations for other phonons by including the vertex function obtained by the X-method-see Section 2.3.

(2) The Phonon Raman Scattering. The phonon Raman scattering gives an indirect evidence for importance of EPI in cuprates [184-188]. We enumerate some of themsee more in [6] and references therein. (i) There is a pronounced asymmetric line-shape (of the Fano resonance) in the metallic state. For instance, in $\mathrm{YBa}_{2} \mathrm{Cu}_{3} \mathrm{O}_{7}$ two Raman modes at $115 \mathrm{~cm}^{-1}$ (Ba dominated mode) and at $340 \mathrm{~cm}^{-1}$ 


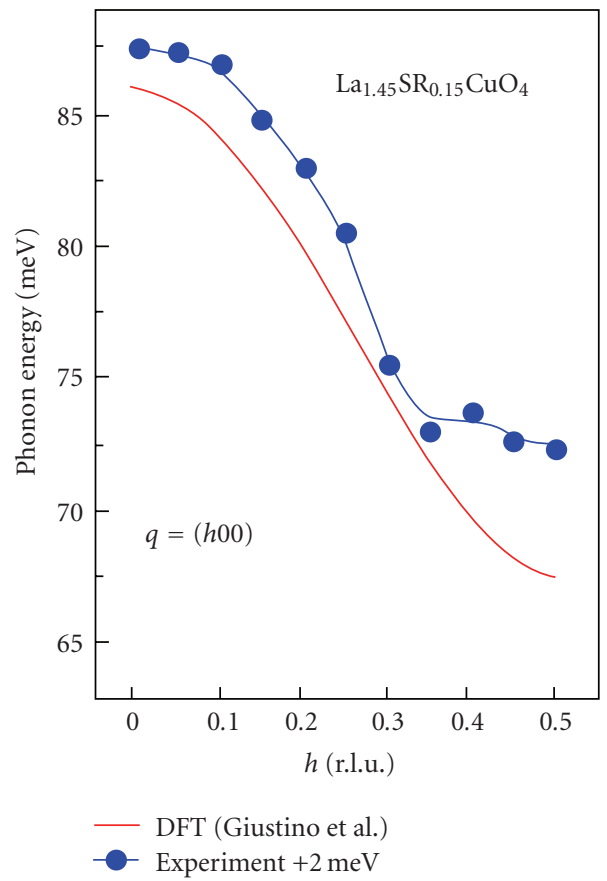

(a)

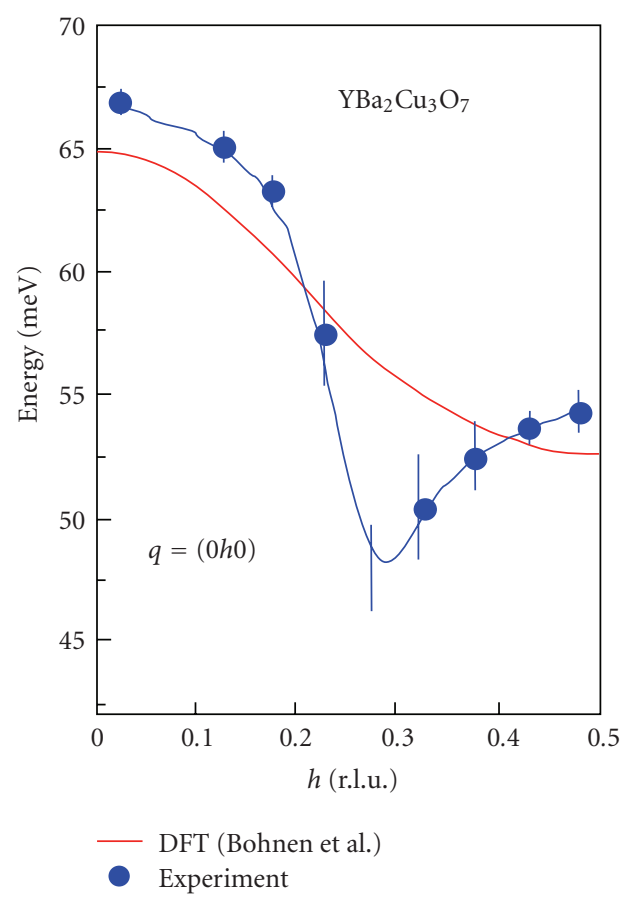

(b)

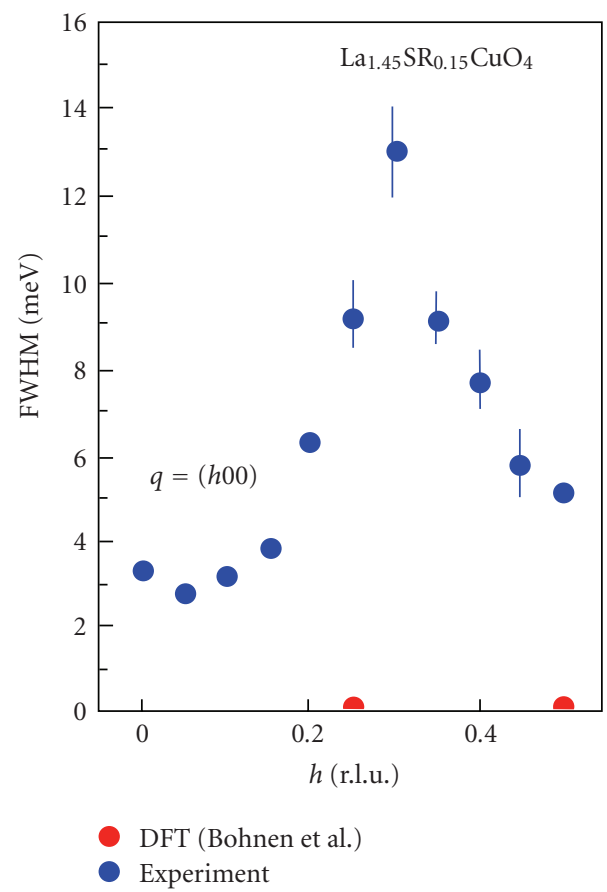

(c)

FIgURE 33: Comparison of DFT calculations with experimental results of inelastic X-ray scattering: (a) phonon energies in $\mathrm{La}_{1.85} \mathrm{Sr}_{0.15} \mathrm{CuO}_{4}$ and (b) in $\mathrm{YBa}_{2} \mathrm{Cu}_{3} \mathrm{O}_{7}$; (c) phonon line-widths in $\mathrm{La}_{1.85} \mathrm{Sr}_{0.15} \mathrm{CuO}_{4}$. DFT calculations [28] give much smaller width than experiments [173175]. From [178].

( $\mathrm{O}$ dominated mode in the $\mathrm{CuO}_{2}$ planes) show pronounced asymmetry which is absent in $\mathrm{YBa}_{2} \mathrm{Cu}_{3} \mathrm{O}_{6}$. This asymmetry means that there is an appreciable interaction of Raman active phonons with continuum states (quasiparticles). (ii) The phonon frequencies for some $A_{1 g}$ and $B_{1 g}$ are strongly renormalized in the superconducting state, between (6-10)\%, pointing again to the importance of EPI [188]see also $[6,37,38]$. To this point we mention that there is a 
remarkable correlation between the electronic Raman crosssection $\widetilde{S}_{\exp }(\omega)$ and the optical conductivity in the $a-b$ plane $\sigma_{a b}(\omega)$, that is, $\tilde{S}_{\exp }(\omega) \sim \sigma_{a b}(\omega)$ [6]. In previous subsections it is argued that EPI with the very broad spectral function $\alpha^{2} F(\omega)(0<\omega \lesssim 80 \mathrm{meV})$ explains in a natural way the $\omega$ and $T$ dependence of $\sigma_{a b}(\omega)$. This means that the electronic Raman spectra in cuprates can be explained by EPI in conjunction with strong correlations. This conclusion is supported by the calculations of the Raman cross-section [189] which take into account EPI with $\alpha^{2} F(\omega)$ extracted from the tunnelling measurements in $\mathrm{YBa}_{2} \mathrm{Cu}_{3} \mathrm{O}_{6+x}$ and $\mathrm{Bi}_{2} \mathrm{Sr}_{2} \mathrm{CaCu}_{2} \mathrm{O}_{8+x}[6,42-54]$. Quite similar properties (to cuprates) of the electronic Raman scattering, as well as of $\sigma(\omega), R(\omega)$, and $\rho(T)$, were observed in experiments [108] on isotropic 3D metallic oxides $\mathrm{La}_{0.5} \mathrm{Sr}_{0.5} \mathrm{CoO}_{3}$ and $\mathrm{Ca}_{0.5} \mathrm{Sr}_{0.5} \mathrm{RuO}_{3}$ where there are no signs of antiferromagnetic fluctuations. This means that low dimensionality and antiferromagnetic spin fluctuations cannot be a prerequisite for anomalous scattering of quasiparticles and EPI must be inevitably taken into account since it is present in all these compounds.

1.3.6. Isotope Effect in $T_{c}$ and $\Sigma(k, \omega)$. The isotope effect $\alpha_{T_{C}}$ in the critical temperature $T_{c}$ was one of the very important proofs for the EPI pairing mechanism in low-temperature superconductors (LTSCs). As a curiosity the isotope effect in LTSC systems was measured almost exclusively in monoatomic systems and in few polyatomic systems: the hydrogen isotope effect in $\mathrm{PdH}$, the Mo and Se isotope shift of $T_{c}$ in $\mathrm{Mo}_{6} \mathrm{Se}_{8}$, and the isotope effect in $\mathrm{Nb}_{3} \mathrm{Sn}$ and $\mathrm{MgB}_{2}$. We point out that very small $\left(\alpha_{T_{C}} \approx 0\right.$ in $\mathrm{Zr}$ and $\mathrm{Ru}$ ) and even negative (in PdH) isotope effects in some polyatomic systems of LTSC materials are compatible with the EPI pairing mechanism but in the presence of substantial Coulomb interaction or lattice anharmonicity. The isotope effect $\alpha_{T_{C}}$ cannot be considered as the smoking gun effect since it is sensitive to numerous influences. For instance, in $\mathrm{MgB}_{2}$ it is with certainty proved that the pairing is due to EPI and strongly dominated by the boron vibrations, but the boron isotope effect is significantly reduced, that is, $\alpha_{T_{C}} \approx 0.3$ and the origin for this smaller value is still unexplained. The situation in HTSC cuprates is much more complicated because they are strongly correlated systems and contain many atoms in unit cell. Additionally, the situation is complicated with the presence of intrinsic and extrinsic inhomogeneities, low dimensionality which can mask the isotope effects. On the other hand new techniques such as ARPES, STM, and $\mu S R$ allow studies of the isotope effects in quasiparticle self-energies, that is, $\alpha_{\Sigma}$, which will be discussed below.

(1) Isotope Effect $\alpha_{T_{C}}$ in $T_{c}$. This problem will be discussed only briefly since more extensive discussion can be found in [6]. It is well known that in the pure EPI pairing mechanism the total isotope coefficient $\alpha$ is given by $\alpha_{T_{C}}=\sum_{i, p} \alpha_{i}^{(p)}=$ $-\sum_{i, p} d \ln T_{c} / d \ln M_{i}^{(p)}$, where $M_{i}^{(p)}$ is the mass of the $i$ th element in the $p$ th crystallographic position. We stress that the total isotope effect is not measured in HTSC cuprates but only some partial ones. Note that, in the case when the screened Coulomb interaction is negligible, that is, $\mu_{c}^{*}=0$, the theory predicts $\alpha_{T_{C}}=1 / 2$. From this formula one can deduce that the relative change of $T_{c}, \delta T_{c} / T_{c}$, for heavier elements should be rather small-for instance, it is 0.02 for ${ }^{135} \mathrm{Ba} \rightarrow{ }^{138} \mathrm{Ba}, 0.03$ for ${ }^{63} \mathrm{Cu} \rightarrow{ }^{65} \mathrm{Cu}$, and 0.07 for ${ }^{138} \mathrm{La} \rightarrow{ }^{139} \mathrm{La}$. This means that the measurements of $\alpha_{i}$ for heavier elements are confronted with the ability of the present experimental techniques. Therefore most isotope effect measurements were done by substituting light atoms ${ }^{16} \mathrm{O}$ by ${ }^{18} \mathrm{O}$ only. It turns out that in most optimally doped HTSC cuprates $\alpha_{\mathrm{O}}$ is rather small. For instance, $\alpha_{\mathrm{O}} \approx$ 0.02-0.05 in $\mathrm{YBa}_{2} \mathrm{Cu}_{3} \mathrm{O}_{7}$ with $T_{c, \text { max }} \approx 91 \mathrm{~K}$, but it is appreciable in $\mathrm{La}_{1.85} \mathrm{Sr}_{0.15} \mathrm{CuO}_{4}$ with $T_{c, \text { max }} \approx 35 \mathrm{~K}$ where $\alpha_{\mathrm{O}} \approx 0.1-0.2$. In $\mathrm{Bi}_{2} \mathrm{Sr}_{2} \mathrm{CaCu}_{2} \mathrm{O}_{8}$ with $T_{c, \text { max }} \approx 76 \mathrm{~K}$ one has $\alpha_{\mathrm{O}} \approx 0.03-0.05$ while $\alpha_{\mathrm{O}} \approx 0.03$ and even negative $(-0.013)$ in $\mathrm{Bi}_{2} \mathrm{Sr}_{2} \mathrm{Ca}_{2} \mathrm{Cu}_{2} \mathrm{O}_{10}$ with $T_{c, \max } \approx 110 \mathrm{~K}$. The experiments on $\mathrm{Tl}_{2} \mathrm{Ca}_{n-1} \mathrm{BaCu}_{n} \mathrm{O}_{2 n+4}(n=2,3)$ with $T_{c, \text { max }} \approx 121 \mathrm{~K}$ are still unreliable and $\alpha_{\mathrm{O}}$ is unknown. In the electron-doped $\left(\mathrm{Nd}_{1-x} \mathrm{Ce}_{x}\right)_{2} \mathrm{CuO}_{4}$ with $T_{c, \text { max }} \approx 24 \mathrm{~K}$ one has $\alpha_{\mathrm{O}}<0.05$ while in the underdoped materials $\alpha_{\mathrm{O}}$ increases. The largest $\alpha_{\mathrm{O}}$ is obtained even in the optimally doped compounds like in systems with substitution, such as $\mathrm{La}_{1.85} \mathrm{Sr}_{0.15} \mathrm{Cu}_{1-x} \mathrm{M}_{x} \mathrm{O}_{4}$, $M=\mathrm{Fe}, \mathrm{Co}$, where $\alpha_{\mathrm{O}} \approx 1.3$ for $x \approx 0.4 \%$. In $\mathrm{La}_{2-x} \mathrm{M}_{x} \mathrm{CuO}_{4}$ there is a $\mathrm{Cu}$-isotope effect which is of the order of the oxygen one, that is, $\alpha_{\mathrm{Cu}} \approx \alpha_{\mathrm{O}}$ giving $\alpha_{\mathrm{Cu}}+\alpha_{\mathrm{O}} \approx 0.25-0.35$ for optimally doped systems $(x=0.15)$. In case when $x=0.125$ with $T_{c} \ll T_{c, \max }$ one has $\alpha_{\mathrm{Cu}} \approx 0.8-1$ with $\alpha_{\mathrm{Cu}}+\alpha_{\mathrm{O}} \approx 1.8[190,191]$. The appreciable copper isotope effect in $\mathrm{La}_{2-x} \mathrm{M}_{x} \mathrm{CuO}_{4}$ tells us that vibrations other than oxygen ions are important in giving high $T_{c}$. In that sense one should have in mind the tunnelling experiments discussed above, which tell us that all phonons contribute to the Eliashberg pairing function $\alpha^{2} F(\mathbf{k}, \omega)$ and according to these results the oxygen modes give moderate contribution to $T_{c}[53,54]$. Hence the small oxygen isotope effect $\alpha_{T_{c}}^{(\mathrm{O})}$ in optimally doped cuprates, if it is an intrinsic property at all (due to pronounced local inhomogeneities of samples and quasi-two-dimensionality of the system), does not exclude the EPI mechanism of pairing.

(2) Isotope Effect $\alpha_{\Sigma}$ in the Self-Energy. The fine structure of the quasiparticle self-energy $\Sigma(\mathbf{k}, \omega)$, such as kinks and slopes, can be resolved in ARPES measurements and in some respect in STM measurements. It turns out that there is isotope effect in the self-energy in the optimally doped Bi2212 samples [139, 141, 142]. In the first paper on this subject [139] it is reported a red shift $\delta \omega_{k, 70} \sim$ $-(10-15) \mathrm{meV}$ of the nodal kink at $\omega_{k, 70} \simeq 70 \mathrm{meV}$ for the ${ }^{16} \mathrm{O} \rightarrow{ }^{18} \mathrm{O}$ substitution. In [139] it is reported that the isotope shift of the self-energy $\delta \Sigma=\Sigma_{16}-\Sigma_{18} \sim 10 \mathrm{meV}$ is very pronounced at large energies $\omega=100-300 \mathrm{meV}$. Concerning the latter result, there is a dispute since it is not confirmed in other experiments [141, 142]. However, the isotope effect in $\operatorname{Re} \Sigma(\mathbf{k}, \omega)$ at low energies [141, 142] is well described in the framework of the Migdal-Eliashberg theory for EPI [140] which is in accordance with the recent ARPES measurements with low-energy photons $\sim 7 \mathrm{eV}$ [192]. The 


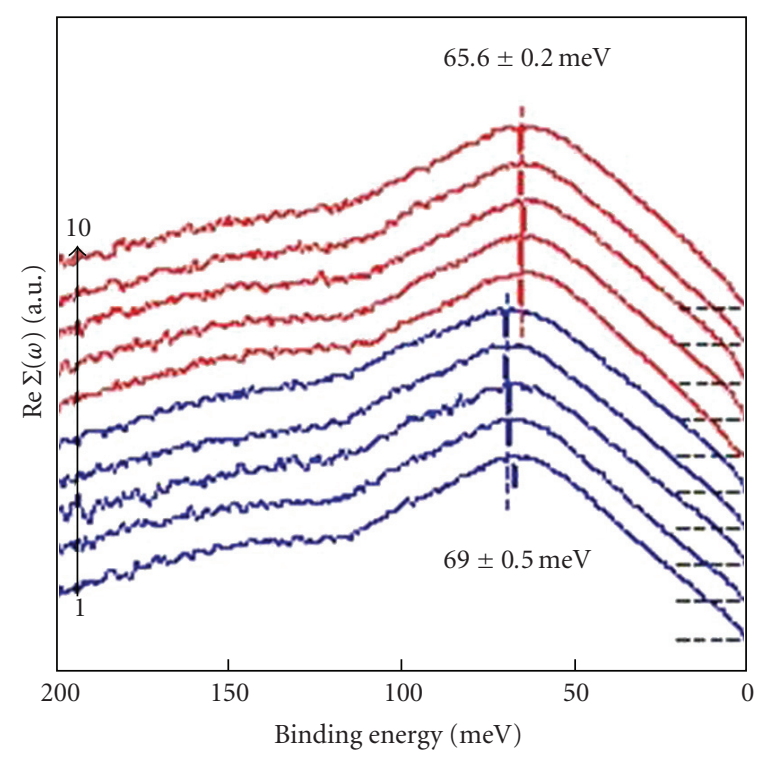

(a)

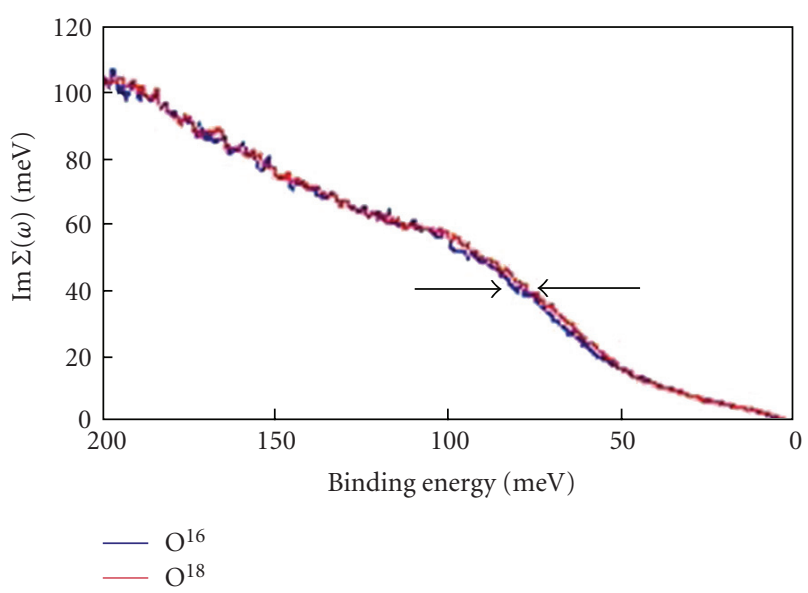

(b)

FIgURe 34: (a) Effective Re $\Sigma$ for five samples for $\mathrm{O}^{16}$ (blue) and $\mathrm{O}^{18}$ (red) along the nodal direction. (b) Effective $\operatorname{Im} \Sigma$ determined from MDC full widths. An impurity term is subtracted at $\omega=0$. From [192].

latter allowed very good precision in measuring the isotope effect in the nodal point of Bi-2212 with $T_{c}^{16}=92.1 \mathrm{~K}$ and $T_{c}^{18}=91.1 \mathrm{~K}$ [192]. They observed a shift in the maximum of $\operatorname{Re} \Sigma\left(\mathbf{k}_{N}, \omega\right)$ - at $\omega_{k, 70} \approx 70 \mathrm{meV}$ (it corresponds to the half-breathing or to the breathing phonon)-by $\delta \omega_{k, 70} \approx$ $3.4 \pm 0.5 \mathrm{meV}$ as shown in Figure 34 .

By analyzing the shift in $\operatorname{Im} \Sigma\left(\mathbf{k}_{N}, \omega\right)$-shown in Figure 34-one finds similar result for $\delta \omega_{k, 70} \approx 3.2 \pm$ $0.6 \mathrm{meV}$. The similar shift was obtained in STM measurements [61-63] which is shown in Figure 32(b) and can have its origin in different phonons. We would like to stress two points: (i) in compounds with $T_{c} \sim 100 \mathrm{~K}$ the oxygen isotope effect in $T_{c}$ is moderate, that is, $\alpha_{T_{c}}^{(\mathrm{O})}<0.1$ [192]. If we consider this value to be intrinsic, then even in this case it is not in conflict with the tunnelling experiments [53,
54] since the latter give evidence that vibrations of heavier ions contribute significantly to $T_{c}$ - see the discussion in Subsection 1.3.4 on the tunnelling spectroscopy. (ii) In ARPES measurements of [192] the effective EPI coupling constant $\lambda_{\text {ep,eff }} \gtrsim 0.6$ is extracted, while the theory in Subsection 1.3.3 gives that the real coupling constant is larger, that is, $\lambda_{e p}>1.2$. This value is significantly larger than the LDA-DFT theory predicts $\lambda_{e p, \mathrm{LDA}}<0.3[28,29]$. This again points that the LDA-DFT method does not pick up the many-body effects due to strong correlations-see Section 2.

1.4. Summary of Section 1. The analysis of experimental data in HTSC cuprates which are related to optics, tunnelling, and ARPES measurements near and at the optimal doping gives evidence for the large electron-phonon interaction (EPI) with the coupling constant $1<\lambda_{e p}<3.5$. We stress that this analysis is done in the framework of the Migdal-Eliashberg theory for EPI which is a reliable approach for systems near the optimal doping. The spectral function $\alpha^{2} F(\omega)$, averaged over the Fermi surface, is extracted from various tunnelling measurements on bulk materials and tin films. It contains peaks at the same energies as the phonon density of states $F_{\mathrm{ph}}(\omega)$. So obtained spectral function when inserted in the Eliashberg equations provides sufficient strength for obtaining high critical temperature $T_{c} \sim 100 \mathrm{~K}$. These facts are a solid proof for the important role of EPI in the normal-state scattering and pairing mechanism of cuprates. Such a large (experimental) value of the EPI coupling constant and the robustness of the $d$-wave superconductivity in the presence of impurities imply that the EPI potential and the impurity scattering amplitude must be strongly momentum dependent. The IR optical reflectivity data provide additional but indirect support for the importance of EPI since by using the spectral function (extracted from tunnelling measurements) one can quantitatively explain frequency dependence of the dynamical conductivity, optical relaxation rate, and optical mass. These findings related to EPI are additionally supported by ARPES measurements on BSCO compounds. The ARPES kinks, the phononic features and the isotope effect in the quasiparticle self-energy in the nodal and antinodal points at low energies $\left(\omega \ll \omega_{c}\right)$ persist in the normal and superconducting state. They are much more in favor of EPI than for the spin fluctuation (SFI) scattering mechanism. The transport EPI coupling constant in HTSC cuprates is much smaller than $\lambda_{e p}$, that is, $\lambda_{\mathrm{tr}} \sim$ $\lambda_{e p} / 3$, which points to some peculiar scattering mechanism not met in low-temperature superconductors. The different renormalization of the quasiparticle and transport selfenergies by the Coulomb interaction (strong correlations) hints to the importance of the small-momentum scattering in EPI. This will be discussed in Section 2.

The ineffectiveness of SFI to solely provide pairing mechanism in cuprates comes out also from the magnetic neutron scattering on $\mathrm{YBCO}$ and BSCO. As a result, the imaginary part of the susceptibility is drastically reduced in the low-energy region by going from slightly underdoped toward optimally doped systems, while $T_{c}$ is practically unchanged. This implies that the real SFI coupling constant $\lambda_{\text {sf }}\left(\sim g_{\text {sf }}^{2}\right)$ is small since the experimental value $g_{\text {sf }}^{(\exp )}<0.2 \mathrm{eV}$ 
is much smaller than the assumed theoretical value $g_{\text {sf }}^{\text {(th) }} \approx$ $(0.7-1.5) \mathrm{eV}$.

Inelastic neutron and X-ray scattering measurements in HTSC cuprates show that the broadening of some phonon lines is by an order of magnitude larger than the LDA-DFA methods predict. Since the phonon line-widths depend on the EPI coupling and the charge susceptibility, it is evident that calculations of both quantities are beyond the range of applicability of LDA-DFT. As a consequence, the LDA-DFT calculations overestimate the electronic screening and thus underestimate the EPI coupling, since many-body effects due to strong correlations are not contained in this mean-field theory. However, in spite of the promising and encouraging experimental results about the dominance of EPI in cuprates, the theory is still confronted with difficulties in explaining sufficiently large coupling constant in the $d$-channel. At present there is not such a satisfactory microscopic theory although some concepts, such as the the dominant EPI scattering at small transfer momenta, are understood at least qualitatively. This set of problems and questions will be discussed in Section 2.

\section{Theory of EPI in HTSC}

The experimental results in Section 1 give evidence that the electron-phonon interaction (EPI) in HTSC cuprates is strong and in order to be conform with $d$-wave pairing EPI must be peaked at small transfer momenta. A number of other experiments in HTSC cuprates give evidence that these are strongly correlated systems with large on-site Coulomb repulsion of electrons on the $\mathrm{Cu}$-ions. However, at present there is no satisfactory microscopic theory of pairing in HTSC cuprates which is able to calculate $T_{c}$ and the order parameter. This is due to mathematical difficulties in obtaining a solution of the formally exact $a b$ initio many-body equations which take into account two important ingredients-EPI and strong correlations [6]. In Section 2.1 we discuss first the ab initio many-body theory of superconductivity in order to point places which are most difficult to be solved. Since the superconductivity is low energy phenomenon (also in HTSC cuprates), one can simplify the structure of the $\mathrm{ab}$ initio equations in the low-energy sector (the Migdal-Eliashberg theory), where the highenergy processes are incorporated in the (so called) ideal band-structure (nonlocal) potential $V_{\mathrm{IBS}}(\mathbf{x}, \mathbf{y})$ and the vertex function $\Gamma$. This program of calculations of $V_{\mathrm{IBS}}(\mathbf{x}, \mathbf{y}), \Gamma$, and the EPI coupling (matrix elements) $g_{e p}(\mathbf{x}, \mathbf{y})$ is not realized in HTSC superconductors due to its complexity. However, one pragmatical way out is to calculate $g_{e p}$ in the framework of the LDA-DFT method which is at present stage unable to treat strong correlations in a satisfactory manner. Some achievements and results of the LDA-DFT methods which are related to HTSC cuprates are discussed in Section 2.2.

In the case of very complicated systems, such as the HTSC cuprates, the standard (pragmatical) procedure in physics is to formulate a minimal theoretical model-sometimes called toy model—which includes minimal set of important ingredients necessary for qualitative and semiquantitative study of a phenomenon. As a consequence of the experimental results, the minimal theoretical model must comprise two important ingredients: (1) EPI and (2) strong correlations. In Section 2.3 we will formulate such a minimal theoretical model-called the $t-J$ model which includes EPI too. In the framework of this model we will discuss the renormalization of EPI by strong correlations. In recent years the interest in these problems is increased and numerous numerical calculations were done mostly on small clusters with $n \times n$ atoms $(n<8)$. We will not discuss this subject which is fortunately covered in the recent comprehensive review in $[10,11]$. The analytical approaches in studying the renormalization of EPI by strong correlations, which are based on a controllable and systematic theory, are rather scarce. We will discuss such a systematic and controllable theory in the framework of the $t-J$ model with $E P I$, which is formulated and solved in terms of Hubbard operators. The theory of this (toy) model predicts some interesting effects which might be important for understanding the physics of HTSC cuprates. It predicts that the high-energy processes (due to the suppression of doubly occupancy for $U \gg W_{b}$ ) give rise to a nonlocal contribution to the band-structure potential (self-energy $\Sigma(\mathbf{x}, \mathbf{y}, \omega=0))$ as well as to EPI. This nonlocality in EPI is responsible for the peak in the effective pairing potential $\left(V_{e p \text {,eff }}(\mathbf{q}, \omega)\right.$ ) at small transfer momenta $q\left(\leqslant q_{c} \ll k_{F}\right)[6,78-80,130]$. The latter property allows that the (strong) EPI is conform with $d$-wave pairing in HTSC cuprates. Furthermore, the peculiar structural properties of HTSC cuprates and corresponding electronic quasi-twodimensionality give an additional nonlocality in EPI. The latter is due to the change of the weakly screened Madelung energy which is involved in most of the lattice vibrations along the $c$-axis. Since at present there is no quantitative theory for the latter effect, we tackle this problem here only briefly. The next task for the future studies of the physics of HTSC cuprates is to incorporate these structural properties in the minimal theoretical $t-J$ model.

Finally, by writing this chapter our intention is not to overview the theoretical studies of EPI in HTSC cuprateswhich is an impossible task-but first to elucidate the descending way from the (old) well-defined ab initio microscopic theory of superconductivity to the one of the minimal model which treats the interplay of EPI and strong correlations. Next, we would like to encourage the reader to further develop the theory of HTSC cuprates.

\subsection{Microscopic Theory of Superconductivity}

2.1.1. Ab Iniitio Many-Body Theory. The many-body theory of superconductivity is based on the fully microscopic electron-ion Hamiltonian for electrons and ions in the crystal—see, for instance, $[193,194]$. It comprises mutually interacting electrons which interact also with the periodic lattice and with the lattice vibrations. In order to pass continually to the problem of the interplay of EPI and strong correlations and also to explain why the LDA-DFT method is inadequate for HTSC cuprates, we discuss this problem here with restricted details-more extended discussion can be 
found in $[6,194]$. In order to describe superconductivity the Nambu-spinor $\hat{\psi}^{\dagger}(\mathbf{r})=\left(\hat{\psi}_{\dagger}^{\dagger}(\mathbf{r}) \hat{\psi}_{!}(\mathbf{r})\right)$ is introduced which operates in the electron-hole space $\left(\hat{\psi}(\mathbf{r})=\left(\hat{\psi}^{\dagger}(\mathbf{r})\right)^{\dagger}\right)$ where $\hat{\psi}_{\uparrow}(\mathbf{r}), \hat{\psi}_{\uparrow}^{\dagger}(\mathbf{r})$ are annihilation and creation operators for spin up, respectively, and so forth. The microscopic Hamiltonian of the system under consideration contains three parts: $\hat{H}=$ $\hat{H}_{e}+\hat{H}_{i}+\hat{H}_{e-i}$. The electronic Hamiltonian $\hat{H}_{e}$, which describes the kinetic energy and the Coulomb interactions of electrons, is given by

$$
\begin{aligned}
\hat{H}_{e}= & \int d^{3} r \hat{\psi}^{\dagger}(\mathbf{r}) \hat{\tau}_{3} \epsilon_{0}(\hat{p}) \hat{\psi}(\mathbf{r}) \\
& +\frac{1}{2} \int d^{3} r d^{3} r^{\prime} \hat{\psi}^{\dagger}(\mathbf{r}) \hat{\tau}_{3} \hat{\psi}(\mathbf{r}) V_{c}\left(\mathbf{r}-\mathbf{r}^{\prime}\right) \hat{\psi}^{\dagger}\left(\mathbf{r}^{\prime}\right) \hat{\tau}_{3} \hat{\psi}\left(\mathbf{r}^{\prime}\right),
\end{aligned}
$$

where $\epsilon_{0}(\hat{p})=\hat{p}^{2} / 2 m$ is the kinetic energy of electron and $V_{c}\left(\mathbf{r}-\mathbf{r}^{\prime}\right)=e^{2} /\left|\mathbf{r}-\mathbf{r}^{\prime}\right|$ is the electron-electron Coulomb interaction. Note that in the electron-hole space the pseudospin (Nambu) matrices $\hat{\tau}_{i}, i=0,1,2,3$ are Pauli matrices. Since we will discuss only the electronic properties, the explicit form of the lattice Hamiltonian $\hat{H}_{i}[6,194]$ is omitted here. The electron-ion Hamiltonian describes the interaction of electrons with the equilibrium lattice and with its vibrations, respectively:

$$
\begin{aligned}
\hat{H}_{e-i}= & \sum_{n} \int d^{3} r V_{e-i}\left(\mathbf{r}-\mathbf{R}_{n}^{0}\right) \hat{\psi}^{\dagger}(\mathbf{r}) \hat{\tau}_{3} \hat{\psi}(\mathbf{r}) \\
& +\int d^{3} r \widehat{\Phi}(\mathbf{r}) \hat{\psi}^{\dagger}(\mathbf{r}) \hat{\tau}_{3} \hat{\psi}(\mathbf{r}) .
\end{aligned}
$$

Here, $V_{e-i}\left(\mathbf{r}-\mathbf{R}_{n}^{0}\right)$ is the electron-ion potential and its form depends on the level of description of the electronic subsystem. For instance, in the all-electron calculations one has $V_{e-i}\left(\mathbf{r}-\mathbf{R}_{n}^{0}\right)=-Z e^{2} /\left|\mathbf{r}-\mathbf{R}_{n}^{0}\right|$ where $Z e$ is the ionic charge. The second term which is proportional to the lattice distortion operator $\widehat{\Phi}(\mathbf{r})=-\sum_{n, \alpha} \hat{u}_{\alpha n} \nabla_{\alpha} V_{e-i}(\mathbf{r}-$ $\left.\mathbf{R}_{n}^{0}\right)+\hat{\Phi}_{\text {anh }}(\mathbf{r})$ (because of convenience it includes also the EPI coupling $\left.\nabla_{\alpha} V_{e-i}\right)$ describes the interaction of electrons with harmonic $\left(\sim \hat{u}_{\alpha n}\right)$ (or anharmonic $\left.\sim \widehat{\Phi}_{\text {anh }}(\mathbf{r})\right)$ lattice vibrations.

Dyson's equations for the electron and phonon Green's functions $\hat{G}(1,2)=-\left\langle T \hat{\psi}(1) \hat{\psi}^{\dagger}(2)\right\rangle, \widetilde{D}(1-2)=$ $-\langle T \hat{\Phi}(1) \hat{\Phi}(2)\rangle$ are $\hat{G}^{-1}(1,2)=\hat{G}_{0}^{-1}(1,2)-\widehat{\Sigma}(1,2)$ and $\widetilde{D}^{-1}(1,2)=\widetilde{D}_{0}^{-1}(1,2)-\widetilde{\Pi}(1,2)$, where the $\hat{G}_{0}^{-1}(1,2)=$ $\left[\left(-\partial / \partial \tau_{1}-\epsilon_{0}\left(\mathbf{p}_{1}\right)+\mu\right) \hat{\tau}_{0}-u_{\text {eff }}(1) \hat{\tau}_{3}\right] \delta(1-2)$ is the bare inverse electronic Green's function. Here, $1=\left(\mathbf{r}_{1}, \tau_{1}\right)$, where $\tau_{1}$ is the imaginary time in the Matsubara technique, and the effective one-body potential $u_{\text {eff }}(1)=V_{e-i}(1)+V_{H}+\langle\widehat{\Phi}(1)\rangle$, where $V_{H}$ is the Hartree potential. The electron and phonon selfenergies $\hat{\Sigma}(1,2)$ and $\widetilde{\Pi}(1,2)$ take into account many-body dynamics of the interacting system. The electronic self-energy $\widehat{\Sigma}(1,2)=\widehat{\Sigma}_{c}(1,2)+\widehat{\Sigma}_{e p}(1,2)$ is obtained in the form

$$
\widehat{\Sigma}(1,2)=-V_{\text {eff }}(1, \overline{1}) \hat{\tau}_{3} \hat{G}(1, \overline{2}) \widehat{\Gamma}_{\text {eff }}(\overline{2}, 2 ; \overline{1}),
$$

where integration (summation) over the bar indices is understood. The effective retarded potential $V_{\text {eff }}(1, \overline{1})$ in $(48)$ contains the screened (by the electron dielectric function $\left.\varepsilon_{e}(1,2)\right)$ Coulomb and EPI interactions:

$$
V_{\text {eff }}(1,2)=V_{c}(1-\overline{1}) \varepsilon_{e}^{-1}(\overline{1}, 2)+\varepsilon_{e}^{-1}(1, \overline{1}) \widetilde{D}(\overline{1}, \overline{2}) \varepsilon_{e}^{-1}(\overline{2}, 2) .
$$

The inverse electronic dielectric permeability $\varepsilon_{e}^{-1}(1,2)$ $=\delta(1-2)+V_{c}(1-\overline{1}) P(\overline{1}, \overline{2}) \mathcal{E}_{e}^{-1}(\overline{2}, 2)$ is defined via the irreducible electronic polarization operator $P(1,2)$ $=-S p\left\{\hat{\tau}_{3} \hat{G}(1, \overline{2}) \hat{\Gamma}_{\text {eff }}(\overline{2}, \overline{3} ; 2) \hat{G}\left(\overline{3}, 1^{+}\right)\right\}$. The vertex function $\widehat{\Gamma}_{\text {eff }}(1,2 ; 3)=-\delta \hat{G}(1,2) / \delta u_{\text {eff }}(3)$ in $(48)$ is the solution of the complicated (and practically unsolvable) integro-differential functional equation

$$
\begin{aligned}
\widehat{\Gamma}_{\text {eff }}(1,2 ; 3)= & \hat{\tau}_{3} \delta(1-2) \delta(1-3) \\
& +\frac{\delta \hat{\Sigma}(1,2)}{\delta \hat{G}(\overline{1}, \overline{2})} \hat{G}(\overline{1}, \overline{3}) \hat{G}(\overline{4}, \overline{2}) \widehat{\Gamma}_{\text {eff }}(\overline{3}, \overline{4} ; 3) .
\end{aligned}
$$

Note that the effective vertex function $\widehat{\Gamma}_{\text {eff }}(1,2 ; 3)$, which takes into account all renormalizations going beyond the simple Coulomb (RPA) screening, is the functional of both the electronic and phononic Green's functions $\hat{G}$ and $\widetilde{D}$, thus making at present the ab initio microscopic equations practically unsolvable.

2.1.2. Low-Energy Migdal-Eliashberg Theory. If the vertex function $\hat{\Gamma}_{\text {eff }}$ would be known, we would have a closed set of equations for Green's functions which describe dynamics of the interacting electrons and lattice vibrations (phonons) in the normal and superconducting state. However, this is a formidable task and at present far from any practical realization. Fortunately, we are mostly interested in lowenergy phenomena (with energies $\left|\omega_{n}\right|, \xi \ll \omega_{c}$ and for momenta $k=k_{F}+\delta k$ in the shell $\delta k \ll \delta k_{c}$ near the Fermi momentum $k_{F} ; \omega_{c}$ and $\delta k_{c}$ are some cutoffs), which allows us further simplification of equations $[1,2]$. Therefore, the strategy is to integrate high-energy processes-see more in [194]. Here, we sketch this procedure briefly. Namely, Green's function $\hat{G}\left(\mathbf{k}, \omega_{n}\right)=\left[i \omega_{n}-\left(\mathbf{k}^{2} / 2 m-\mu\right) \widehat{\tau}_{3}-\hat{\Sigma}\left(\mathbf{k}, \omega_{n}\right)\right]^{-1}$ can be formally written in the form

$$
\hat{G}\left(\mathbf{k}, \omega_{n}\right)=\hat{G}^{\text {low }}\left(\mathbf{k}, \omega_{n}\right)+\hat{G}^{\text {high }}\left(\mathbf{k}, \omega_{n}\right),
$$

where $\hat{G}^{\text {low }}\left(\mathbf{k}, \omega_{n}\right)=\hat{G}\left(\mathbf{k}, \omega_{n}\right) \Theta\left(\omega_{c}-\left|\omega_{n}\right|\right) \Theta\left(\delta k_{c}-\delta k\right)$ is the low-energy Green's function and $\hat{G}^{\text {high }}\left(\mathbf{k}, \omega_{n}\right)=$ $\hat{G}\left(\mathbf{k}, \omega_{n}\right) \Theta\left(\left|\omega_{n}\right|-\omega_{c}\right) \Theta\left(\delta k-\delta k_{c}\right)$ is the high-energy one and analogously $D=D^{\text {low }}\left(\mathbf{k}, \omega_{n}\right)+D^{\text {high }}\left(\mathbf{k}, \omega_{n}\right)$. By introducing the small parameter of the theory $s \sim\left(\omega / \omega_{c}\right) \sim\left(\delta k / \delta k_{c}\right) \ll 1$ one has in leading order $\hat{G}^{\text {low }}\left(\mathbf{k}, \omega_{n}\right) \sim s^{-1}, \hat{G}^{\text {high }}\left(\mathbf{k}, \omega_{n}\right) \lesssim$ 1 and $D^{\text {low }}\left(\mathbf{k}, \omega_{n}\right) \sim s^{0}, D^{\text {high }}\left(\mathbf{k}, \omega_{n}\right) \sim s^{2}$. Note that the coupling constants $\left(V_{e i}, \nabla V_{e i}, V_{i i}\right.$, etc. $)$ are of the order $s^{0}=$ 1.

The procedure of separating low-energy and highenergy processes lies also behind the adiabatic approximation since in most materials the characteristic phonon (Debye) energy $\omega_{D}$ of lattice vibrations is much smaller than the characteristic electronic Fermi energy $E_{F}\left(\omega_{D} \ll E_{F}\right)$. In the 
small $s(\ll 1)$ limit the Migdal theory $[1,2]$ keeps in the total self-energy $\Sigma$ linear terms in the phonon propagator $\tilde{D}(D)$ only. In that case the effective vertex function can be written in the form $\widehat{\Gamma}_{\text {eff }}(1,2 ; 3) \cong \widehat{\Gamma}_{c}(1,2 ; 3)+\delta \widehat{\Gamma}_{e p}(1,2 ; 3)$ $[1,2]$, where the Coulomb charge vertex $\widehat{\Gamma}_{c}(1,2 ; 3)=\hat{\tau}_{3} \delta(1-$ 2) $\delta(1-3)+\delta \hat{\Sigma}_{c}(1,2) / \delta u_{\text {eff }}(3)$ contains correlations due to the Coulomb interaction only but does not contain EPI and phonon propagator $\tilde{D}$ explicitly. The part $\delta \widehat{\Gamma}_{e p}(1,2 ; 3)=$ $\delta \widehat{\Sigma}_{e p}(1,2) / \delta u_{\text {eff }}(3)$ contains all linear terms with respect to EPI. Note that in these diagrams enters the dressed Green's function which contains implicitly EPI up to infinite order. By careful inspection of all (explicit) contributions to $\delta \widehat{\Gamma}_{e p}(1,2 ; 3)$ which is linear in $\widetilde{D}$ one can express the selfenergy in terms of the charge (Coulomb) vertex $\widehat{\Gamma}_{c}(1,2 ; 3)$ only. As a result of this approximation, the part of the selfenergy due to Coulomb interaction is given by

$$
\widehat{\Sigma}_{c}(1,2)=-V_{c}^{\mathrm{sc}}(1, \overline{1}) \hat{\tau}_{3} \hat{G}(1, \overline{2}) \widehat{\Gamma}_{c}(\overline{2}, 2 ; \overline{1}),
$$

where $V_{c}^{s c}(1,2)=V_{c}(1, \overline{2}) \varepsilon_{e}^{-1}(\overline{2}, 2)$ is the screened Coulomb interaction. The part which is due to EPI has the following form:

$$
\widehat{\Sigma}_{e p}(1,2)=-V_{e p}(\overline{1}, \overline{2}) \widehat{\Gamma}_{c}(1, \overline{3} ; \overline{1}) \hat{G}(\overline{3}, \overline{4}) \widehat{\Gamma}_{c}(\overline{4}, 2 ; \overline{2}),
$$

where $V_{e p}(1,2)=\varepsilon_{e}^{-1}(1, \overline{1}) \tilde{D}(\overline{1}, \overline{2}) \varepsilon_{e}^{-1}(\overline{2}, 2)$ is the screened $E P I$ potential. Note that $\hat{\Sigma}_{e p}(1,2)$ depends now quadratically on the charge vertex $\hat{\Gamma}_{c}$, which is due to the adiabatic theorem.

It is well known that the Coulomb self-energy $\hat{\Sigma}_{c}(1,2)$ is the most complicating part of the electronic dynamics, but since we are interested in low-energy physics when $s \ll 1$, then the term $\hat{\Sigma}_{c}(1,2)$ can be further simplified by separating it in two parts:

$$
\widehat{\Sigma}_{c}(1,2)=\widehat{\Sigma}_{c}^{(h)}(1,2)+\widehat{\Sigma}_{c}^{(l)}(1,2) .
$$

The term $\hat{\Sigma}_{c}^{(h)}(1,2)$ is due to high-energy processes contained in the product $\hat{G}^{\text {high }}(1, \overline{2}) \hat{\Gamma}_{c}^{\text {high }}(\overline{2}, 2 ; \overline{1})$ (e.g., due to the large Hubbard $U$ in strongly correlated systems) and $\hat{\Sigma}_{c}^{(l)}(1,2)$ is due to low-energy processes. The leading part of $\hat{\Sigma}_{c}^{(h)}(1,2)$ is 1 , that is, $\hat{\Sigma}_{c}^{(h)}(1,2) \sim s^{0}$, while $\hat{\Sigma}_{c}^{(l)}(1,2)$ is small of order 1 , that is, $\hat{\Sigma}_{c}^{(l)}(1,2) \sim s^{1}$. For further purposes we define the quantity $\widehat{V}_{0}$ as

$$
\widehat{V}_{0}(1,2)=\left\{V_{e-i}(1)+V_{H}(1)\right\} \tau_{3} \delta(1-2)+\hat{\Sigma}_{c}^{(h)}(1,2),
$$

where $V_{e-i}, V_{H}$ are also of order $s^{0}$. After the Fourier transform with respect to time (and for small $\left|\omega_{n}\right| \ll \omega_{c}$ ) $\hat{\Sigma}_{c}^{(h)}$ is given by

$$
\widehat{\Sigma}_{c}^{(h)}\left(\mathbf{x}_{1}, \mathbf{x}_{2}, \omega_{n}\right) \simeq \Sigma_{c 0}^{(h)}\left(\mathbf{x}_{1}, \mathbf{x}_{2}, 0\right) \widehat{\tau}_{3}+\left(\widehat{\Sigma}_{c 0}^{(h)}\right)^{\prime}\left(\mathbf{x}_{1}, \mathbf{x}_{2}, 0\right) \cdot i \omega_{n} .
$$

As we said, $\Sigma_{c 0}^{(h)} \sim s^{0}$ while $\left(\widehat{\Sigma}_{c 0}^{(h)}\right)^{\prime} \cdot \omega_{n} \sim s^{1}$ because $\omega_{n} \sim s^{1}$. From (52) it is seen that the part $\hat{\Sigma}_{c}^{(l)}(1,2)$ contains the lowenergy Green's function $\hat{G}^{\text {low }}(1, \overline{2})$ and this skeleton diagram is of order $s^{1}$. The similar analysis based on $(53)$ for $\hat{\Sigma}_{e p}(1,2)$ gives that the leading order is $s^{1}$ which describes the lowenergy part of EPI. After the separations of terms (of $s^{0}$ and $s^{1}$ orders) the Dyson equation in the low-energy region has the form

$$
\begin{aligned}
& {\left[i \omega_{n} Z_{c}(\mathbf{x}, \overline{\mathbf{x}})-\hat{H}_{0}(\mathbf{x}, \overline{\mathbf{x}})-\hat{\Sigma}_{c}^{(l)}\left(\mathbf{x}, \overline{\mathbf{x}}, \omega_{n}\right)-\hat{\Sigma}_{e p}\left(\mathbf{x}, \overline{\mathbf{x}}, \omega_{n}\right)\right]} \\
& \quad \times \hat{G}^{\text {low }}\left(\overline{\mathbf{x}}, \mathbf{y}, \omega_{n}\right)=\delta(\mathbf{x}-\mathbf{y}) \hat{\tau}_{0},
\end{aligned}
$$

where $\overline{\mathbf{x}}$ means integration $\int d^{3} \bar{x}$ over the crystal volume. The Coulomb renormalization function $Z_{c}(\mathbf{x}, \mathbf{y})=\delta(\mathbf{x}-$ $\mathbf{y})-\left(\Sigma_{0 c}^{(h)}\right)^{\prime}(\mathbf{x}, \mathbf{y}, 0)$ and the single-particle Hamiltonian $\hat{H}_{0}(\mathbf{x}, \mathbf{y})$ collect formally all high-energy processes which are unaffected by superconductivity (which is low-energy process) where

$$
\hat{H}_{0}(\mathbf{x}, \mathbf{y})=\left\{\left(-\frac{1}{2 m} \nabla_{\mathbf{x}}^{2}-\mu\right) \delta(\mathbf{x}-\mathbf{y})+V_{0}^{(h)}(\mathbf{x}, \mathbf{y}, 0)\right\} \hat{\tau}_{3}
$$

with

$$
V_{0}^{(h)}(\mathbf{x}, \mathbf{y}, 0)=\left\{V_{e-i}(\mathbf{x})+V_{H}(\mathbf{x})\right\} \delta(\mathbf{x}-\mathbf{y})+\Sigma_{c 0}^{(h)}(\mathbf{x}, \mathbf{y}, 0) .
$$

One can further absorb $Z_{c}(\mathbf{x}, \mathbf{y})$ into the renormalized Green's function

$$
\hat{G}_{r}\left(x, \mathbf{y}, \omega_{n}\right)=Z_{c}^{1 / 2}(\mathbf{x}, \overline{\mathbf{x}}) \hat{G}^{\text {low }}\left(\bar{x}, \overline{\mathbf{y}}, \omega_{n}\right) Z_{c}^{1 / 2}(\overline{\mathbf{y}}, \mathbf{y}),
$$

the renormalized vertex function $\hat{\Gamma}_{\text {ren }}(1,2 ; 3)=$ $Z_{c}^{-1 / 2} \widehat{\Gamma}_{c} Z_{c}^{-1 / 2}$, and the renormalized self-energies

$$
\widehat{\Sigma}_{r ; c, e p}^{(l)}\left(\mathbf{x}, \mathbf{y}, \omega_{n}\right)=Z_{c}^{-1 / 2}(\mathbf{x}, \overline{\mathbf{x}}) \hat{\Sigma}_{c, e p}^{(l)}\left(\bar{x}, \overline{\mathbf{y}}, \omega_{n}\right) Z_{c}^{-1 / 2}(\overline{\mathbf{y}}, \mathbf{y})
$$

and introduce the ideal band-structure Hamiltonian $\hat{h}_{0}(x, \mathbf{y})=Z_{c}^{-1 / 2}(\mathbf{x}, \overline{\mathbf{x}}) \hat{H}_{0}(\bar{x}, \overline{\mathbf{y}}) Z_{c}^{-1 / 2}(\overline{\mathbf{y}}, \mathbf{y})$ given by

$$
\hat{h}_{0}(\mathbf{x}, \mathbf{y})=\left\{\left(-\frac{1}{2 m} \nabla_{\mathbf{x}}^{2}-\mu\right) \delta(\mathbf{x}-\mathbf{y})+V_{\mathrm{IBS}}(\mathbf{x}, \mathbf{y})\right\} \hat{\tau}_{3} .
$$

Here,

$$
V_{\mathrm{IBS}}(\mathbf{x}, \mathbf{y})=Z_{c}^{-1 / 2}(\mathbf{x}, \overline{\mathbf{x}}) V_{0}^{(h)}(\bar{x}, \overline{\mathbf{y}}) Z_{c}^{-1 / 2}(\overline{\mathbf{y}}, \mathbf{y})
$$

is the ideal band-structure potential (sometimes called the excitation potential) and apparently nonlocal quantity, which is contrary to the standard local potential $V_{g}(\mathbf{x})$ in the LDA-DFT theories-see Section 2.2. The static potential $V_{\text {IBS }}(\mathbf{x}, \mathbf{y})$ is of order $s^{0}$ and includes high-energy processes.

Finally, we obtain the matrix Dyson equation for the renormalized Green's function $\widehat{G}_{r}\left(x, \mathbf{y}, \omega_{n}\right)$ which is the basis for the (strong-coupling) Migdal-Eliashberg theory in the low-energy region

$$
\begin{aligned}
{\left[i \omega_{n} \delta(\mathbf{x}\right.} & \left.-\overline{\mathbf{x}})-\hat{h}_{0}(\mathbf{x}, \overline{\mathbf{x}})-\hat{\Sigma}_{c, r}^{(l)}\left(\mathbf{x}, \overline{\mathbf{x}}, \omega_{n}\right)-\hat{\Sigma}_{e p, r}\left(\mathbf{x}, \overline{\mathbf{x}}, \omega_{n}\right)\right] \\
\times & \hat{G}_{r}\left(\overline{\mathbf{x}}, \mathbf{y}, \omega_{n}\right)=\delta(\mathbf{x}-\mathbf{y}) \hat{\tau}_{0},
\end{aligned}
$$


where $\widehat{\Sigma}_{c, r}^{(l)}$ and $\hat{\Sigma}_{e p, r}$ have the same form as (52)-(53) but with the renormalized Green's and vertex functions $\hat{G}_{r}, \widehat{\Gamma}_{r}$ instead of $\hat{G}, \hat{\Gamma}$. We stress that (64) holds in the low-energy region only. In the superconducting state the set of Eliashberg equations in (64) are written explicitly in Appendix A, where it is seen that the superconducting properties depend on the Eliashberg spectral function $\alpha_{\mathrm{kp}}^{2} F(\omega)$. The latter function is defined also in Appendix A, (A.4), and it depends on material properties of the system.

The important ingredients of the low-energy MigdalEliashberg theory are the ideal band-structure Hamiltonian $\hat{h}_{0}(\mathbf{x}, \mathbf{y})$ - given by (62) which contains many-body (excitation) ideal band-structure nonlocal periodic crystal potential $V_{\text {IBS }}(\mathbf{x}, \mathbf{y})$. The Hamiltonian $\hat{h}_{0}(\mathbf{x}, \mathbf{y})$ determines the ideal energy spectrum $\epsilon(\mathbf{k})$ of the conduction electrons and the wave function $\psi_{i, \mathbf{p}}(\mathbf{x})$ through

$$
\hat{h}_{0}(\mathbf{x}, \overline{\mathbf{y}}) \psi_{i, \mathbf{k}}(\overline{\mathbf{y}})=\left[\epsilon_{i}(\mathbf{k})-\mu\right] \psi_{i, \mathbf{k}}(\mathbf{x}),
$$

where $\mu$ is the chemical potential. We stress that the Hamiltonian $\hat{h}_{0}(x, y)$ also governs transport properties of metals in low-energy region.

After solving (65) the next step is to expand all renormalized Green's function, self-energies, vertices, and the renormalized EPI matrix element (written symbolically as $\left.g_{e p, r}=g_{e p, 0} \Gamma_{\text {ren }} \varepsilon_{e}^{-1}\right)$ in the basis of $\psi_{i, \mathbf{p}}(\mathbf{x})$ and to write down the Eliashberg equations in this basis. We will not elaborate further this program and refer the reader to the relevant literature in $[193,194]$. We point out that even such simplified program of the low-energy MigdalEliashberg theory was never fully realized in low-temperature superconductors, because the nonlocal potential $V_{\mathrm{IBS}}(\mathbf{x}, \mathbf{y})$ (enters the ideal band-structure Hamiltonian $h_{0}(x, y)$ ) and the renormalized vertex function (entering the EPI coupling constant $g_{e p, r}$ ) which include electronic correlations are difficult to calculate especially in strongly correlated metals. Therefore, it is not surprising at all that the situation is even more difficult in HTS materials which are strongly correlated systems with complex structural and material properties. Due to these difficulties the calculations of the electronic band structure and the EPI coupling are usually done in the framework of LDA-DFT where the manybody excitation potential $V_{\mathrm{IBS}}(\mathbf{x}, \mathbf{y})$ is replaced by some (usually local) potential $V_{\mathrm{LDA}}(\mathbf{x})$ which in fact determines the ground-state properties of the crystal. In the next section we briefly describe (i) the LDA-DFT procedure in calculating the EPI coupling constant and (ii) some results of the LDA-DFT calculations related to HTSC cuprates. We will also discuss why this approximation is inappropriate when applied to HTS materials.

2.2. LDA-DFT Calculations of the EPI Matrix Elements. We point out again two results which are important for the future microscopic theory of pairing in HTSC cuprates. First, numerous experiments (discussed in Part I) give evidence that the EPI coupling constant which enters the normal part of the quasiparticle self-energy $\lambda_{e p}^{Z}=\lambda_{s}+\lambda_{d}+\cdots$ is rather large, that is, $1<\lambda_{e p}^{Z}<3.5$. In order to be conform with $d$-wave pairing the effective EPI potential must be nonlocal (and peaked at small transfer momenta $q$ ), which implies that the $s$-wave and $d$-wave coupling constants are of the same order, that is, $\lambda_{d} \approx \lambda_{s}$. Second, the theory based on the minimal $t-J$ model, which will be discussed in Section 2.3, gives that strong electronic correlations produce a peak at small transfer momenta in the effective EPI pairing potential thus giving rise to $\lambda_{d} \approx \lambda_{s}$. This is a striking property which allows that EPI is conform with $d$-wave pairing. However, the theory is seriously confronted with the problem of calculation of the coupling constants $\lambda_{e p}^{Z}$. It turns out that at present it is an illusory task to calculate $\lambda_{e p}^{Z}$ and $\lambda_{d}$ since it is extremely difficult (if possible at all) to incorporate the peculiar structural properties of HTSC cuprates (layered structure, ionic-metallic system, etc.) and strong correlations effects in a consistent and reliable microscopic theory which is described in Section 2.1. As it is stressed several times, the LDA-DFT methods miss some important many-body effects (especially in the band-structure potential) and therefore fail to describe correctly screening properties of HTSC cuprates and the strength of EPI. However, the LDA-DFT methods are able to incorporate diverse structural properties of HTSC cuprates much better than the simplified minimal $t-J$ (toy) model. Here, we discuss briefly some achievements of the advanced LDA-DFT calculations which are able to take partially into account some nonlocal effects in the EPI. The latter are mainly due to the almost ionic structure along the $c$-axis which is reflected in the very small $c$-axis plasma frequency $\left(\omega_{c} \ll \omega_{a b}\right)$.

The main task of the LDA-DFT theory in obtaining the EPI matrix elements is to calculate the change of the groundstate (self-consistent) potential $\delta V_{g}(\mathbf{r}) / \delta R_{\alpha}$ and the EPI coupling constant (matrix element) $g_{\alpha}^{\mathrm{LDA}}\left(\mathbf{k}, \mathbf{k}^{\prime}\right)$ (see its definition below), which is the most difficult part of calculations. Since in the LDA-DFT method the EPI scattering cannot be formulated, then the recipe is that the calculated $g_{\alpha}^{\mathrm{LDA}}\left(\mathbf{k}, \mathbf{k}^{\prime}\right)$ is inserted into the many-body Eliashberg equations. By knowing $g_{\alpha}^{\mathrm{LDA}}\left(\mathbf{k}, \mathbf{k}^{\prime}\right)$ one can define the total $(\lambda)$ and partial $\left(\lambda_{\mathbf{q}, v}\right)$ EPI coupling constants for the $\nu$ th mode, respectively [195], as

$$
\lambda=\frac{1}{N p} \sum_{\mathbf{q}, v} \lambda_{\mathbf{q}, v}, \quad \lambda_{\mathbf{q}, v}=\frac{p \gamma_{\mathbf{q}, v}}{\pi N(0) \omega_{\mathbf{q}, v}},
$$

where $p=3 \kappa$ is the number of phonon branches ( $\kappa$ is the number of atoms in the unit cell) and $N(0)$ is the density of states at the Fermi energy (per spin and unit cell). The phonon line-width $\gamma_{\mathbf{q}, v}$ is defined in the Migdal-Eliashberg theory by

$$
\begin{aligned}
\gamma_{\mathbf{q}, v}= & 2 \pi \omega_{\mathbf{q}, v} \frac{1}{N} \sum_{l l^{\prime} \mathbf{k}} \frac{1}{2 M \omega_{\mathbf{k}-\mathbf{q}, v}}\left|e_{\nu}^{\alpha}(\mathbf{q}) \cdot g_{\alpha, l l^{\prime}}(\mathbf{k}, \mathbf{k}-\mathbf{q})\right|^{2} \\
& \times\left[\frac{n_{F}\left(\xi_{l, \mathbf{k}}\right)-n_{F}\left(\xi_{l, \mathbf{k}}+\hbar \omega_{\mathbf{q}, \nu}\right)}{\hbar \omega_{\mathbf{q}, v}}\right] \\
& \times \delta\left(\xi_{l^{\prime}, \mathbf{k}-\mathbf{q}}-\xi_{l, \mathbf{k}}-\hbar \omega_{\mathbf{q}, v}\right) .
\end{aligned}
$$

Here, $e_{\nu}^{\alpha}(\mathbf{q})$ is the phonon polarization vectors; $n_{F}$ is the Fermi function. Since the ideal energy spectrum from (65) 
$\xi_{l, \mathbf{k}}=E_{l, \mathbf{k}}-\mu$ and the corresponding eigenfunctions $\psi_{\mathbf{k} l}$ are unknown, then instead of these one sets in (67) the LDADFT eigenvalues for the $l$ th band $\xi_{l, \mathbf{k}}^{(\mathrm{LDA})}$ and $\psi_{\mathbf{k} l}^{(\mathrm{LDA})}$. In the LDA-DFT method the EPI matrix element $g_{\alpha, l l^{\prime}}^{(\mathrm{LDA})}$ is defined by the change of the ground-state potential $\delta V_{g}(\mathbf{r}) / \delta R_{\alpha}$ :

$$
g_{\alpha, l l^{\prime}}^{(\mathrm{LDA})}\left(\mathbf{k}, \mathbf{k}^{\prime}\right)=\left\langle\psi_{\mathbf{k} l}^{(\mathrm{LDA})}\left|\sum_{n} \frac{\delta V_{g}(\mathbf{r})}{\delta R_{n \alpha}}\right| \psi_{\mathbf{k}^{\prime} l^{\prime}}^{(\mathrm{LDA})}\right\rangle .
$$

The index $n$ means summation over the lattice sites; $\alpha=$ $x, y, z$ and the wave function $\psi_{\mathbf{k} l}^{(\mathrm{LDA})}$ are the solutions of the Kohn-Sham equation-see [6]. In the past various approximations within the $L D A-D F T$ method have been used in calculating $\delta V_{g}(\mathbf{r}) / \delta R_{\alpha}$ and $\lambda$ while here we comment some of them only. (i) In most calculations in LTS systems and in HTSC cuprates the rigid-ion (RI) approximation was used as well as its further simplifications which inevitably (due to its shortcomings and obtained small $\lambda$ ) deserves to be commented. The $R I$ approximation is based on the very specific assumption that the ground-state (crystal) potential $V_{g}(\mathbf{r})$ can be considered as a sum of ionic potentials $V_{g}(\mathbf{r})=$ $\sum_{n} V_{g}\left(\mathbf{r}-\mathbf{R}_{n}\right)$ where the ion potential $V_{g}\left(\mathbf{r}-\mathbf{R}_{n}\right)$ and the electron density $\rho_{e}(\mathbf{r})$ are carried rigidly with the ion at $\mathbf{R}_{n}$ during the ion displacement $\left(\mathbf{R}_{n}=\mathbf{R}_{n}^{0}+\hat{u}_{\alpha n}\right)$. In the $R I$ approximation the change of $V_{g}(\mathbf{r})$ is given by

$$
\begin{gathered}
\delta V_{g}(\mathbf{r})=\sum_{n} \nabla_{\alpha} V_{g}\left(\mathbf{r}-\mathbf{R}_{n}^{0}\right) u_{\alpha n}, \\
\frac{\delta V_{g}(\mathbf{r})}{\delta R_{n \alpha}}=\nabla_{\alpha} V_{g}\left(\mathbf{r}-\mathbf{R}_{n}^{0}\right),
\end{gathered}
$$

which means that $R I$ does not take into account changes of the electron density during the ion displacements. In numerous calculations applied to HTSC cuprates the rigidion model is even further simplified by using the rigid muffintin approximation (RMTA) (or similar version with the rigidatomic sphere)—see discussions in [195-198]. The RMTA assumes that the ground-state potential and the electron density follow ion displacements rigidly inside the WignerSeitz cell while outside it $V_{g}(\mathbf{r})$ is not changed because of the assumed very good metallic screening (e.g., in simple metals):

$$
\nabla_{\alpha} V_{g}\left(\mathbf{r}-\mathbf{R}_{n}\right)= \begin{cases}\nabla_{\alpha} V_{g}\left(\mathbf{r}-\mathbf{R}_{n}\right), & \mathbf{r} \text { in cell } n \\ 0, & \text { outside. }\end{cases}
$$

This means that the dominant EPI scattering is due to the nearby atoms only and that the scattering potential is isotropic. All nonlocal effects related to the interaction of electrons with ions far away are neglected in the RMTA. In this case $g_{\alpha, n}^{\mathrm{LDA}}\left(\mathbf{k}, \mathbf{k}^{\prime}\right)$ is calculated by the wave function centered at the given ion $\mathbf{R}_{n}^{0}$ which can be expanded inside the muffin-tin sphere (outside it the potential is assumed to be constant) in the angular momentum basis $\{l, m\}$, that is,

$$
\left\langle\mathbf{r} \mid \psi_{\mathbf{k}}^{(\mathrm{RMTA})}\right\rangle=\sum_{l m n} C_{l m}\left(k, \mathbf{R}_{n}^{0}\right) \rho_{l}\left(\left|\mathbf{r}-\mathbf{R}_{n}^{0}\right|\right) Y_{l m}(\phi, \theta)
$$

(the angles $\phi, \theta$ are related to the vector $\hat{r}=\left(\mathbf{r}-\mathbf{R}_{n}^{0}\right) / \mid \mathbf{r}-$ $\left.\mathbf{R}_{n}^{0} \mid\right)$. The radial function $\rho_{l}\left(\left|\mathbf{r}-\mathbf{R}_{n}^{0}\right|\right)$ is zero outside the muffin-tin sphere. In that case the EPI matrix element is given by $g_{\alpha, n}^{\mathrm{RMTA}}\left(\mathbf{k}, \mathbf{k}^{\prime}\right) \sim\left\langle Y_{l m}|\hat{r}| Y_{l^{\prime} m^{\prime}}\right\rangle$ and because $\hat{r}$ is vector the selection rule implies that only terms with $\Delta l \equiv$ $l^{\prime}-l= \pm 1$ contribute to the EPI coupling constant in the RMTA. This result is an immediate consequence of the assumed locality of the EPI potential in RMTA. However, since nonlocal effects, such as the long-range Madelunglike interaction, are important in HTSC cuprates, then additional terms contribute also to the coupling constant $g_{\alpha, n}$, that is, $g_{\alpha, n}\left(\mathbf{k}, \mathbf{k}^{\prime}\right)=g_{\alpha, n}^{\mathrm{RMTA}}\left(\mathbf{k}, \mathbf{k}^{\prime}\right)+g_{\alpha, n}^{\text {nonloc }}\left(\mathbf{k}, \mathbf{k}^{\prime}\right)$, where a part $\left(\delta g_{\alpha, n}^{\text {nonloc }}\right)$ of the nonlocal contribution to $g_{\alpha, n}^{\text {nonloc }}$ is represented schematically:

$$
\delta g_{\alpha, n}^{\text {nonloc }}\left(\mathbf{k}, \mathbf{k}^{\prime}\right) \sim\left\langle Y_{l m}\left|\left(\mathbf{R}_{n}^{0}-\mathbf{R}_{m}^{0}\right)_{\alpha}\right| Y_{l^{\prime} m^{\prime}}\right\rangle .
$$

From (72) comes out the selection rule $\Delta l=l^{\prime}-l=0$ for the nonlocal part of the $E-P$ interaction. We stress that the $\Delta l=0$ (nonlocal) terms are omitted in the RMTA approach and therefore it is not surprising that this approximation works satisfactorily in elemental (isotropic) metals only. The latter are characterized by the large density of states at the Fermi surface which makes electronic screening very efficient. This gives rise to a local EPI where an electron feels potential changes of the nearby atom only. One can claim with certainty that the RMTA method is not suitable for HTSC cuprates which are highly anisotropic systems with pronounced ionic character of binding and pronounced strong electronic correlations. The RMTA method applied to HTSC cuprates misses just this important part-the longrange part EPI due to the change of the long-range Madelung energy in the almost ionic structure of HTSC cuprates. For instance, the first calculations done in [199] which are based on the RMTA give very small EPI coupling constant $\lambda^{\text {RMTA }} \sim$ 0.1 in $Y B C O$, which is in apparent contradiction with the experimental finding that $\lambda_{e p}$ is large-see Section 1.

However, these nonlocal effects are taken into account in [195] by using the frozen-in phonon (FIP) method in evaluating of $\lambda_{e p}$ in $\mathrm{La}_{2-x} \mathrm{M}_{x} \mathrm{CuO}_{4}$. In this method some symmetric phonons are considered and the band structure is calculated for the system with the super-cell which is determined by the periodicity of the phonon displacement. By comparing the unperturbed and perturbed energies the corresponding EPI coupling $\lambda_{v}$ (for the considered phonon $v$ th mode) is found. More precisely speaking, in this approach the matrix elements of $\delta V_{g}(\mathbf{r}) / \delta R_{0, \alpha}^{\kappa}$ are determined from the finite difference of the ground-state potential $\Delta V_{g, \mathbf{q}, \nu}(\mathbf{r})=V_{g}\left(\mathbf{R}_{0, L}^{\kappa}+\right.$ $\left.\Delta \boldsymbol{\tau}_{\mathbf{q}, v}^{\kappa}(L)\right)-V_{g}\left(\mathbf{R}_{0, L}^{\kappa}\right)=\sum_{L, \kappa} \Delta \boldsymbol{\tau}_{\mathbf{q}, v}^{\kappa}(L) \partial V_{g}\left(\mathbf{R}_{0, L}^{\kappa}\right) / \partial \mathbf{R}_{0, L}^{\kappa}$, where $L, \kappa$ enumerate elementary lattice cells and atoms in the unit cell, respectively. The frozen-in atomic displacements of the phonon $\Delta \boldsymbol{\tau}_{\mathbf{q}, \nu}^{\kappa}(L)$ of the $v$ th mode are given by $\Delta \boldsymbol{\tau}_{\mathbf{q}, v}^{\kappa}(L)=\Delta u_{\mathbf{q}, v}\left(\hbar / 2 M_{\kappa} \omega_{\mathbf{q}, v}\right)^{1 / 2} \operatorname{Re}\left[\mathbf{e}_{\kappa, \nu}(\mathbf{q}) e^{i \mathbf{q} \cdot \mathbf{R}}\right]$ where $\Delta u_{\mathbf{q}, v}$ is the dimensionless phonon amplitude and the phonon polarization (eigen)vector $\mathbf{e}_{\kappa, v}(\mathbf{q})$ fulfills the condition $\sum_{\kappa} \mathbf{e}_{\kappa, \nu}^{*}(\mathbf{q}) \cdot \mathbf{e}_{\kappa, v^{\prime}}(\mathbf{q})=\delta_{\nu, v^{\prime}}$. Based on this approach various symmetric $A_{g}$ (and some $B_{3 g}$ ) modes of $\mathrm{La}_{2-x} \mathrm{M}_{x} \mathrm{CuO}_{4}$ were studied [195], where it was found that the large matrix elements are due to unusually long-range Madelung-like, especial for the $c$-axis phonon modes. The obtained large $\lambda_{e p} \approx 1.37$ is the consequence of the following three main 
facts. (i) The electronic spectrum in HTSC cuprates is highly anisotropic, that is, it is quasi-two-dimensional. This is an important fact for pairing because if the conduction electrons would be uniformly spread over the whole unit cell then due to the rather low electron density $\left(n \sim 10^{21} \mathrm{~cm}^{-3}\right)$ the density of states on the $\mathrm{Cu}$ and $\mathrm{O}$ in-plane atoms would be an order of magnitude smaller than the real value. This would further give an order of magnitude smaller EPI coupling constant $\lambda_{e p}$. Note that the calculated density of states on the (heavy) $\mathrm{Cu}$ and (light) $\mathrm{O}$ in-plane atoms, $N^{\mathrm{Cu}}(0) \cong$ 0.54 states/eV and $N^{\mathrm{O}_{x y}}(0) \cong 0.35$ states/eV, is of same order of magnitude as in some LTS materials. For instance, in $\mathrm{NbC}$ where $T_{c} \approx 11 \mathrm{~K}$ one has on (the heavy) $\mathrm{Nb}$ atom $N^{\mathrm{Nb}}(0) \cong 0.58$ states/eV and on (the light) $\mathrm{C}$ atom $N^{\mathrm{C}}(0) \cong$ 0.25 states/eV. So, the quasi-two-dimensional character of the spectrum is crucial in obtaining appreciable density of states on the light $\mathrm{O}$ atoms in the $\mathrm{CuO}_{2}$ planes. (ii) In HTSC cuprates there is strong $\mathrm{Cu}-\mathrm{O}$ hybridization leading to good in-plane metallic properties. This large covalency in the plane is due to the (fortunately) small energy separation of the electron levels on $\mathrm{Cu}$ and $\mathrm{O}_{x y}$ atoms which comes out from the band-structure calculations [200], that is, $\Delta=\mid \epsilon_{\mathrm{Cu}}-$ $\epsilon_{\mathrm{O}_{x y}} \mid \approx 3 \mathrm{eV}$. The latter value gives rise to strong covalent mixing (the hybridization parameter $t_{p d}$ ) of the $\mathrm{Cu}_{d_{x^{2}-y^{2}}}$ and $\mathrm{O}_{p_{x, y}}$ states, that is, $t_{p d}=-1.85 \mathrm{eV}$. It is interesting that the small value of $\Delta$ is not due to the ionic structure (crystal field effect) of the system but it is mainly due to the natural falling of the $\mathrm{Cu}_{d_{x^{2}-y^{2}}}$ states across the transitionmetal series. So, the natural closeness of the atomic energy levels of the $\mathrm{Cu}_{d_{x^{2}-y^{2}}}$ and $\mathrm{O}_{p_{x}, p_{y}}$ states is this distinctive feature of HTSC cuprates which basically allows achievement of high $T_{c}$. (iii) The ionic structure of HTSC cuprates which is very pronounced along the $c$-axis is responsible for the weak electronic screening along this axis and according to that for the significant contribution of the nonlocal (longrange) Madelung-like interaction to EPI. It turns out that because of the ionicity of the structure the $\mathrm{La}$ and $\mathrm{O}_{z}$ axial modes are strongly coupled with charge carriers in the $\mathrm{CuO}_{2}$ planes despite the fact that the local density of states on these atoms is very small [195], that is, $N^{\mathrm{La}}(0)=0.13$ states/eV and $N^{\mathrm{O}_{z}}(0)=0.016$ states $/ \mathrm{eV}$. (For comparison, on planar atoms $\mathrm{Cu}$ and $\mathrm{O}_{x y}$ one has $N^{\mathrm{Cu}}(0)=0.54$ states/eV and $N^{\mathrm{O}_{x y}}(0)=$ 0.35 states/eV.) These calculations show that the lanthanum mode (with $\omega_{\mathbf{q}, v}=202 \mathrm{~cm}^{-1}$ ) at the $q=(0,0.2 \pi / c)$ zone boundary (fully symmetric $Z$-point) has ten times larger coupling constant $\lambda_{\mathbf{q}, v}^{\mathrm{La}}(F I P)=4.8$ than it is predicted in the $R M T$ approximation $\lambda_{\mathbf{q}, v}^{\mathrm{La}}(\mathrm{RMT})=0.48$. The similar increase holds for the average coupling constant, where $\lambda_{\nu \text {,average }}^{\mathrm{La}}(\mathrm{FIP})=1.0$ but $\lambda_{\nu \text {,average }}^{\mathrm{La}}(\mathrm{RMT})=0.1$. Note that for the $\mathbf{q} \approx 0$ La-mode one obtains $\lambda_{v}^{\mathrm{La}}($ FIP $)=4.54$ compared to $\lambda_{v}^{\mathrm{La}}(\mathrm{RMT})=0.12$. Similar results hold for the axial apexoxygen $q=(0,0.2 \pi / c)$ mode $\left(\mathrm{O}_{z}\right)$ with $\omega_{\mathbf{q}, v}=396 \mathrm{~cm}^{-1}$ where the large (compared to the RMT method) coupling constant is obtained: $\lambda_{\mathbf{q}, v}^{\mathrm{O}_{z}}=14$ and $\lambda_{\nu \text {,average }}^{\mathrm{O}_{z}}=4.7$, while for $q \approx 0$ axial apex-oxygen modes with $\omega_{\mathbf{q}, v}=415 \mathrm{~cm}^{-1}$ one has $\lambda_{v \text {,average }}^{\mathrm{O}_{z}}=11.7$. After averaging over all calculated modes it was estimated that $\lambda=1.37$ and $\omega_{\log } \approx 400 \mathrm{~K}$. By assuming that $\mu^{*}=0.1$ one obtains $T_{c}=49 \mathrm{~K}$ by using
Allen-Dynes formula for $T_{c} \approx 0.83 \omega_{\log } \exp \{-1.04(1+\lambda) /[\lambda-$ $\left.\left.\mu^{*}(1+0.62 \lambda)\right]\right\}$ with $\omega_{\log }=2 \int d \omega d \omega \alpha^{2}(\omega) F(\omega) \ln \omega / \lambda \omega$. For $\mu^{*}=0.15$ and 0.2 one obtains $T_{c}=41$ and $32 \mathrm{~K}$, respectively. We stress that the rather large $\lambda_{e p}$ (and $T_{c}$ ) is due to the nonlocal (long range) effects of the metallic-ionic structure of HTSC cuprates and non-muffin-tin corrections in EPI, as was first proposed in $[201,202]$. However, we would like to stress that the optimistic results for $\lambda_{e p}$ obtained in [195] are in fact based on the calculation of the EPI coupling for some wave vectors $\mathbf{q}$ with symmetric vibration patterns and in fact the obtained $\lambda_{e p}$ is an extrapolated value. The allq calculations of $\lambda_{e p, \mathbf{q}}$ which take into account long-range effects are a real challenge for the LDA-DFT calculations and are still awaiting.

Finally, it is worth to mention important calculations of the EPI coupling constant in the framework of the linear-response full-potential linear-muffin-tin-orbital method (LRFP-LMTO) invented in $[203,204]$ and applied to the doped HTSC cuprate $\left(\mathrm{Ca}_{1-x} \mathrm{Sr}_{x}\right)_{1-y} \mathrm{CuO}_{2}$ for $x \sim 0.7$ and $y \sim 0.1$ with $T_{c}=110 \mathrm{~K}$ [205]. Namely, these calculations give strong evidence that the structural properties of HTSC cuprates already alone make the dominance of small-q scattering in EPI, whose effect is additionally increased by strong correlations. In order to analyze this compound in [205] the calculations are performed for $\mathrm{CaCuO}_{2}$ doped by holes in a uniform, neutralizing back-ground charge. The momentum $\left(\mathbf{q}=\left(\mathbf{q}_{\|}, q_{\perp}\right)\right)$ dependent EPI coupling constant (summed over all phonon branches $v$ ) in different $L$ channels $(s, p, d$.$) is calculated by using a standard expression$

$$
\lambda_{L}(\mathbf{q})=M \sum_{\mathbf{k}, \nu} Y_{L}(\mathbf{k}+\mathbf{q})\left|g_{\mathbf{k}, \mathbf{q}, \nu}\right|^{2} Y_{L}(\mathbf{k}) \delta\left(\xi_{\mathbf{k}+\mathbf{q}}\right) \delta\left(\xi_{\mathbf{k}}\right)
$$

Here, $\xi_{\mathbf{k}}$ is the quasiparticle energy, $g_{\mathbf{k}, \mathbf{q}, v}$ is the EPI coupling constant (matrix element) with the $\nu$ th branch, $Y_{L}(\mathbf{k})$ is the $L$-channel wave function, and the normalization factor $M \propto N_{L}^{-1}(0)$ with the partial density of states is $N_{L}(0) \propto$ $\sum_{\mathbf{k}} Y_{L}^{2}(\mathbf{k}) \delta\left(\xi_{\mathbf{k}}\right)$. The total coupling constant in the $L$-channel is an average of $\lambda_{L}(\mathbf{q})$ over the whole $2 \mathrm{D}$ Brillouin zone (over $\left.\mathbf{q}_{\|}\right)$, that is, $\lambda_{L}\left(q_{\perp}\right)=\left\langle\lambda_{L}\left(\mathbf{q}_{\|}\right)\right\rangle_{\mathrm{BZ}}$. We stress three important results of [205]. First, the $s$ - and $d$-coupling constants, $\lambda_{s}(\mathbf{q})$, $\lambda_{d}(\mathbf{q})$, are peaked at small transfer momenta $\mathbf{q} \sim(\pi / 3 a, 0,0)$ as it is shown in [205, Figure 3]. This result is mainly caused by the nesting properties of the Fermi surface shown in [205, Figure 1]. Second, the q-dependence of the integrated EPI matrix elements $\left|\bar{g}_{L, \mathbf{q}}\right|^{2}=\lambda_{L}(\mathbf{q}) / \chi_{L}^{\prime \prime}(\mathbf{q})$ (with $\chi_{L}^{\prime \prime}(\mathbf{q}) \propto$ $\left.\sum_{\mathbf{k}} Y_{L}(\mathbf{k}+\mathbf{q}) Y_{L}(\mathbf{k}) \delta\left(\xi_{\mathbf{k}+\mathbf{q}}\right) \delta\left(\xi_{\mathbf{k}}\right)\right)$ for $L=s, d$ is similar to that of $\lambda_{L}(\mathbf{q})$, that is, these are peaked at small transfer momenta $q \ll 2 k_{F}$. Both of these results mean that the structural properties of HTSC cuprates imply the dominance of small$q$ EPI scattering. Third, the calculations give similar values for $\lambda_{s}\left(q_{\perp}=0\right)$ and $\lambda_{d}\left(q_{\perp}=0\right)$, that is, $\lambda_{s}=0.47$ for $s$ wave and $\lambda_{d}=0.36$ for $d$-wave pairing [205]. The result that $\lambda_{d} \approx \lambda_{s}$ is due to the dominance of the small $q$-scattering in EPI, which means that the nonlocal effects (long-range forces) in EPI of HTSC cuprates are very important. This result together with the finding of the dominance of the small- $q$ scattering in EPI due to strong correlations [78$80,130,179,180]$ mean that strong correlations and the 
peculiar structural properties of HTSC cuprates make EPI conform with $d$-wave pairing, either as its main cause or as its supporter. We stress that the obtained coupling constant $\lambda_{d}=0.36$ is rather small to give $d$-wave pairing with large $T_{c}$ and on the first glance this result is against the EPI mechanism of pairing in cuprates. However, it is argued throughout this paper that the LDA methods applied to strongly correlated systems overestimate the screening effects and underestimate the coupling constant and therefore their quantitative predictions are not reliable.

\subsection{EPI and Strong Correlations in HTSC Uprates}

2.3.1. Minimal Model Hamiltonian. The minimal microscopic model for HTSC cuprates must include at least three orbitals:

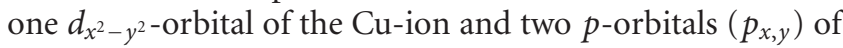
the $\mathrm{O}$-ion since they participate in transport properties of these materials - see more in [6] and references therein. The electronic part of the Hamiltonian (of the minimal model) is $\hat{H}=\hat{H}_{0}+\hat{H}_{\text {int }}$-usually called the Emery model (or the $p$ - $d$ model) [206], where the one-particle tight-binding Hamiltonian $\hat{H}_{0}$ describes the lowering of the kinetic energy in the $p$ - $d$ model (with three bands or orbitals):

$$
\begin{aligned}
\hat{H}_{0}= & \sum_{i, \sigma}\left(\epsilon_{d}^{0}-\mu\right) d_{i \sigma}^{\dagger} d_{i \sigma}+\sum_{j, \alpha, \sigma}\left(\epsilon_{p \alpha}^{0}-\mu\right) p_{j \alpha \sigma}^{\dagger} p_{j \alpha \sigma} \\
& +\sum_{i, j, \alpha, \sigma} t_{i j \alpha}^{p d} d_{i \sigma}^{\dagger} p_{j \alpha \sigma}+\sum_{j, j^{\prime}, \alpha, \beta, \sigma} t_{j j^{\prime}, \alpha \beta}^{p p} p_{j \alpha \sigma}^{\dagger} p_{j^{\prime} \beta \sigma} .
\end{aligned}
$$

Here $t_{i j \alpha}^{p d}$ ( $i, j$ enumerate the $\mathrm{Cu}$ - and O-sites, resp.) is the hopping integral between the $p_{\alpha}(\alpha=x, y)$-and $d$-states and $t_{j j^{\prime} \alpha \beta}^{p p}$ between the $p_{\alpha}$ - and $p_{\beta}$-states - while $\epsilon_{d}^{0}$ and $\epsilon_{p \alpha}^{0}$ are the bare $d$ - and $p$-local energy levels and $\mu$ is the chemical potential. This tight-binding Hamiltonian is written in the electronic notation where the charge-transfer energy $\Delta_{d p, 0} \equiv$ $\epsilon_{d}^{0}-\epsilon_{p}^{0}>0$ by assuming that there is one $3 d_{x^{2}-y^{2}}$ electron on the copper $\left(\mathrm{Cu}^{2+}\right)$ while electrons in the $p$-levels of the $\mathrm{O}^{2-}$ ions occupy filled bands. $\hat{H}_{0}$ contains the main ingredients coming from the comparison with the LDA-DFT bandstructure calculations. The $L D A-D F T$ results are reproduced by assuming that $t^{p p} \ll t_{p d}$ (and $\epsilon_{p \alpha}^{0}=\epsilon_{p}^{0}$ ) where the good fit to the LDA-DFT band structure is found for $\Delta_{d p, 0} \equiv$ $\epsilon_{d}^{0}-\epsilon_{p}^{0} \approx 3.2 \mathrm{eV}$ and $t^{p d}\left(\equiv t_{p d}\right)=(\sqrt{3} / 2)(p d \sigma),(p d \sigma)=$ $-1.8 \mathrm{eV}$. The total LDA bandwidth $W_{b}=(4 \sqrt{2})\left|t_{p d}\right| \cong 9 \mathrm{eV}$ [207].

The electron interaction is described by $\hat{H}_{\text {int }}$ :

$$
\hat{H}_{\mathrm{int}}=U_{d} \sum_{i} n_{i \uparrow}^{d} n_{i \downarrow}^{d}+U_{p} \sum_{j, \alpha} n_{j \alpha \uparrow}^{p} n_{j \alpha \downarrow}^{p}+\widehat{V}_{c}+\widehat{V}_{e p},
$$

where $U_{d}$ and $U_{p}$ are the on-site Coulomb repulsion energies at $\mathrm{Cu}$ and $\mathrm{O}$ sites, respectively, while $\hat{V}_{c}$ and $\hat{V}_{e p}$ describe the long-range part of the Coulomb interaction of electrons (holes) and EPI, respectively. Note that the Hubbard repulsion $U_{d}$ on the $\mathrm{Cu}$-ion is different from its bare atomic value $U_{d 0}(\approx 16 \mathrm{eV}$ for $\mathrm{Cu})$ due to various kinds of screening effects in solids [208-210]. It turns out that in most transition metal oxides one has $U_{d} \ll U_{d 0}$. This problem is thoroughly studied in [208-210] and applied to HTSC cuprates. The estimation from the numerical cluster calculations [211] gives $U_{d}=9-11 \mathrm{eV}$ and $U_{p}=4-6 \mathrm{eV}$ but because $n_{i}^{d} U_{d} \gg n_{j}^{p} U_{p}$ the on-site repulsion on the oxygen ion is usually neglected at the first stage of the analysis.

Note that in the case of large $U_{d}\left(\gg t_{p d}, \Delta_{d p, 0}\right)$ the hole notation is usually used where in the parent compound (and for $\left.\left|t_{p d}\right| \ll \Delta_{d p, 0}\right)$ one has $\left\langle n_{i}^{d}\right\rangle=1$, that is, one hole in the $3 \mathrm{D}$-shell (in the $3 d_{x^{2}-y^{2}}$ state) in the ground state. In the limit of large $U_{d} \rightarrow \infty$ the doubly occupancy on the Cu atoms is forbidden and only two copper states are possible: $\mathrm{Cu}^{2+}$ - described by the quantum state $d_{i \sigma}^{\dagger}|0\rangle$ with one hole in the $3 \mathrm{D}$ shell and $\mathrm{Cu}^{1+}$ _-described by $|0\rangle$ with zero holes in the filled $3 \mathrm{D}$ shell. In this (hole) notation the oxygen $p$ level is fully occupied by electrons, that is, there are no holes $\left(\left\langle n_{j}^{p}\right\rangle=0\right)$ in the occupied oxygen $2 p$-shell of $\mathrm{O}^{2-}$. In this notation the vacuum state $\left|0_{v}\right\rangle$ (not the ground state) of the Hamiltonian $\hat{H}$ for large $U_{d}$ corresponds to the closedshell configuration $\mathrm{Cu}^{1+} \mathrm{O}^{2-}$. In the hole notation the hole $p$-level $\epsilon_{\mathrm{ph}}^{0}$ lies higher than the hole $d$-level $\epsilon_{d h}^{0}$, that is, $\Delta_{p d, 0} \equiv \epsilon_{\mathrm{ph}}^{0}-\epsilon_{d h}^{0}>0$ (note that in the electron notation it is opposite) and $U_{d}$ means repulsion of two holes (in the $3 d_{x^{2}-y^{2}}$ orbital) with opposite spins- $-3 d^{8}$ configuration of the $\mathrm{Cu}^{3+}$ ion. Note that $\epsilon_{\mathrm{ph}}^{0}=-\epsilon_{p}^{0}, \epsilon_{d h}^{0}=-\epsilon_{d}^{0}$, and $t_{p d, h}=-t_{p d}$. In the following the index $h$ in $t_{p d, h}$ is omitted. The reason for $\epsilon_{\mathrm{ph}}^{0}>\epsilon_{d h}^{0}$ is partly in different energies for the hole sitting on the oxygen and copper, respectively [207]. From this model one can derive in the limit $U \rightarrow \infty$ the $t-J$ model for the 2D lattice in the $\mathrm{CuO}_{2}$ plane $[212,213]$, where now each lattice site corresponds to a $\mathrm{Cu}$-atom. In the presence of one hole in the 3D-shell then in the undoped (no oxygen holes) HTSC cuprate each lattice site is occupied by one hole. By doping the system with holes the additional holes go onto O-sites. Furthermore, due to the strong $\mathrm{Cu}-$ $\mathrm{O}$ covalent binding the energetics of the system implies that an O-hole forms a Zhang-Rice singlet with a Cu-hole [212]. In the $t-J$ model the Zhang-Rice singlet is described by an empty site. Since in the $t-J$ model the doubly occupancy is forbidden, one introduces annihilation (Hubbard) operator of the composite fermion $\hat{X}_{i}^{\sigma 0}=c_{i \sigma}^{\dagger}\left(1-n_{i,-\sigma}\right)$ which describes creation of a hole (in the $3 \mathrm{D}$-shell of the $\mathrm{Cu}$-atoms) on the $i$ th site if this site is previously empty (thus excluding doubly occupancy), that is, the constraint $n_{i, \sigma}+n_{i,-\sigma} \leq 1$ must be fulfilled on each lattice site. In this picture the doped-hole concentration $\delta$ means at the same time the concentration of the oxygen holes, that is, of the Zhang-Rice singlets.

In order not to confuse the reader we stress the difference in the meaning of the hole in the $(p-d)$ three-band Emery model and in the single-band (effective) $t$ - $J$ model. In the Emery model the hole means the absence of the electron in the filled shell-the 3D shell for $\mathrm{Cu}$ atoms(ions) and $2 p$ shell for $\mathrm{O}$ atoms(ions). On the other side the hole on the $i$ th lattice site in the $t-J$ model means the presence of the ZhangRice singlet on this site.

The bosonic-like operators $\hat{X}_{i}^{\sigma_{1} \sigma_{2}}=\hat{X}_{i}^{\sigma_{1} 0} \hat{X}_{i}^{0 \sigma_{2}}$ for $\sigma_{1} \neq \sigma_{2}$ create a spin fluctuation at the $i$ th site and the spin operator is 
given by $\mathbf{S}=\hat{X}_{i}^{\bar{\sigma}_{1} 0}(\vec{\sigma})_{\bar{\sigma}_{1} \bar{\sigma}_{2}} \hat{X}_{i}^{0 \bar{\sigma}_{2}}$ where summation over the bar indices is understood. The operator $\sum_{\sigma} \hat{X}_{i}^{\sigma \sigma}$ has the meaning of the hole number on the ith site. It is useful to introduce the operator $\hat{X}_{i}^{00}=\hat{X}_{i}^{0 \sigma} \hat{X}_{i}^{\sigma 0}$ at the $i$ th lattice site which is the number of Zhang-Rice singlets on the ith site. For $\hat{X}_{i}^{00}|0\rangle=$ $1|0\rangle$ the $i$ th site is occupied by the Zhang-Rice singlet, while for $\hat{X}_{i}^{00}|1\rangle=0|1\rangle$ there is no Zhang-Rice singlet on the $i$ th site (i.e., this site is occupied only by one $3 d^{9}$ hole on the $\mathrm{Cu}$ site). This property of $\hat{X}_{i}^{00}$ is due to the local constraint

$$
\hat{X}_{i}^{00}+\sum_{\sigma=\uparrow \downarrow} \hat{X}_{i}^{\sigma \sigma}=1,
$$

which forbids doubly occupancy of the ith site by holes. By projecting out doubly occupied (high-energy) states the $t-J$ model reads

$$
\begin{aligned}
\hat{H}_{t-j}= & \sum_{i, \sigma} \epsilon_{i}^{0} \hat{X}_{i}^{\sigma \sigma}-\sum_{i, j, \sigma} t_{i j} \hat{X}_{i}^{\sigma 0} \hat{X}_{j}^{0 \sigma} \\
& +\sum_{i, j} J_{i j}\left(\mathbf{S}_{i} \cdot \mathbf{S}_{j}-\frac{1}{4} \hat{n}_{i} \hat{n}_{j}\right)+\hat{H}_{3} .
\end{aligned}
$$

The first term $\left(\sim \epsilon_{i}^{0}\right)$ describes an effective local energy of the hole (or the Zhang-Rice singlet), the second one ( $\sim t_{i j}$ ) describes hopping of the holes, and the third one $\left(\sim J_{i j}\right)$ is the Heisenberg-like exchange energy between two holes. The theory [212] predicts that $\left|\epsilon_{i}^{0}\right| \gg\left|t_{i j}\right|$. This property is very important in the study of EPI. $\hat{H}_{3}$ contains three-site term which is usually omitted believing that it is not important. For charge fluctuation processes it is plausible to omit it, while for spin-fluctuation processes it is questionable approximation. If one introduces the enumeration $\alpha, \beta, \gamma, \lambda=0, \uparrow, \downarrow$, then the Hubbard operators satisfy the following algebra:

$$
\left[\hat{X}_{i}^{\alpha \beta}, \hat{X}_{j}^{\gamma \lambda}\right]_{ \pm}=\delta_{i j}\left[\delta_{\gamma \beta} \hat{X}_{i}^{\alpha \lambda} \pm \delta_{\alpha \lambda} \hat{X}_{i}^{\gamma \beta}\right]
$$

where $\delta_{i j}$ is the Kronecker symbol. Note that the Hubbard operators possess the projection properties with $\hat{X}_{i}^{\alpha \beta} \hat{X}_{i}^{\gamma \lambda}=$ $\delta_{\beta \gamma} \hat{X}_{i}^{\alpha \lambda}$. The (anti)commutation relations in (78) are more complicated than the canonical Fermi and Bose (anti)commutation relations, which complicates the mathematical structure of the theory. To escape these complications some novel techniques have been used, such as the one slave boson-technique. In this technique $\hat{X}_{i}^{0 \sigma}=f_{i \sigma} b_{i}^{\dagger}, \hat{X}_{i}^{\sigma_{1} \sigma_{2}}=$ $f_{i \sigma_{1}}^{\dagger} f_{i \sigma_{2}}$ are represented in terms of the fermion (spinon) operator $f_{i \sigma}$ which annihilates the spin on the $i$ th and the boson (holon) operator $b_{i}^{\dagger}$ which creates the Zhang-Rice singlet.

In the minimal theoretical model the electron-phonon interaction (EPI) contains in principle two leading terms:

$$
\hat{H}_{e p}=\hat{H}_{e p}^{\mathrm{ion}}+\hat{H}_{e p}^{\mathrm{cov}} \text {, }
$$

which are the "ionic" one $\left(\hat{H}_{e p}^{\text {ion }}\right)$ and the "covalent" one $\left(\hat{H}_{e p}^{\text {cov }}\right)$. The "ionic" term describes the change of the energy of the hole (or the Zhang-Rice singlet) at the $i$ th site due to lattice vibrations and it reads [6, 78-80, 130]

$$
\hat{H}_{e p}^{\text {ion }}=\sum_{i, \sigma} \hat{\Phi}_{i} \hat{X}_{i}^{\sigma \sigma},
$$

where the "displacement" operator

$$
\widehat{\Phi}_{i}=\sum_{L \kappa}\left[\epsilon\left(\mathbf{R}_{i}^{0}-\mathbf{R}_{L \kappa}^{0}+\widehat{\mathbf{u}}_{i}-\widehat{\mathbf{u}}_{L \kappa}\right)-\epsilon\left(\mathbf{R}_{i}^{0}-\mathbf{R}_{L \kappa}^{0}\right)\right]
$$

(which as in Section 2.1 includes the bare coupling constant) describes the change of the hole (or Zhang-Rice singlet) energy $\epsilon_{a, i}^{0}$ by displacing atoms in the lattice by the vector $\widehat{\mathbf{u}}_{L \kappa}$. In the harmonic approximation the EPI potential is given by $\hat{\Phi}_{i}=\sum g_{i}(\mathbf{q}, \lambda) \exp \left\{i \mathbf{q} \mathbf{R}_{i}\right\}\left[b_{\mathbf{q}, \lambda}+b_{-\mathbf{q}, \lambda}^{\dagger}\right]$ where $b_{\mathbf{q}, \lambda}$ and $b_{\mathbf{q}, \lambda}^{\dagger}$ are the annihilation and creation operator of phonons with the polarization $\lambda$, respectively. This term describes in principle the following processes: (1) the change of the $\mathrm{O}$ hole and $\mathrm{Cu}$-hole bare energies $\epsilon_{\mathrm{ph}}^{0}, \epsilon_{d h}^{0}$ in the three-band model due to lattice vibrations, (2) the change of the longrange Madelung energy (which is due to the ionicity of the structure) by lattice vibrations along the $c$-axis, and (3) the change of the $\mathrm{Cu}-\mathrm{O}$ hopping parameter $t_{p d}$ in the presence of vibrations, and so forth. Here, $L$ and $\kappa$ enumerate unit lattice vectors and atoms in the unit cell, respectively. Usually, the EPI scattering is studied in the harmonic approximation where the phonon operator $\widehat{\Phi}_{i}$ is calculated in the harmonic approximation $(\hat{\Phi} \sim \hat{u})$ for the EPI interaction of holes with some specific phononic modes, such as the breathing and half-breathing ones $[10,11,169]$. The theory which includes also all other (than oxygen) vibrations in $\widehat{\Phi}_{i}$ is still awaiting.

It is interesting to make comparison of the EPI coupling constants in the $t-J$ model and in the Hartree-Fock (HF) approximation (which is the analogous of the LDA-DFT method) of the three-band Emery $(p-d)$ model in (74)(75) when the problem is projected on the single band. For instance, the coupling constant with the half-breathing mode at the zone boundary in the HF approximation (which mimics the LDA-DFT approach) is given by

$$
g_{\mathrm{hb}}^{\mathrm{HF}}= \pm 4 t_{p d} \frac{\partial t_{p d}}{\partial R_{\mathrm{Cu}-\mathrm{O}}} \frac{1}{\epsilon_{d}-\epsilon_{p}} u_{0}
$$

while the coupling constant in the $t-J$ model $g_{\mathrm{hb}}^{t-J}(=$ $\left.\partial \epsilon^{0} / \partial R_{\mathrm{Cu}-\mathrm{O}}\right)$ is given by

$$
g_{\mathrm{hb}}^{t-J}= \pm 4 t_{p d} \frac{\partial t_{p d}}{\partial R_{\mathrm{Cu}-\mathrm{O}}}\left[\frac{2 p^{2}-1}{\epsilon_{d}-\epsilon_{p}}+\frac{2 p^{2}}{U_{d}-\left|\epsilon_{d}-\epsilon_{p}\right|}\right] u_{0},
$$

where $p=0.96$ - see $[10,11,169]$ and references therein. It is obvious that in the $t-J$ model the electron-phonon coupling is different from the HF one, since the former contains an additional term coming from the many-body effects, which are not comprised by the HF (LDA-DFT) calculations. The first term in (83) describes the hopping of a $3 \mathrm{D}$ hole into the $\mathrm{O} 2 p$-states and this term exists also in the LDA-DFT coupling constant-see (82). However, the second term in (83), which is due to many-body effects, describes the 
hopping of an O $2 p$-hole into the (already) single occupied $\mathrm{Cu} 3 \mathrm{D}$ state and it does not exist in the LDA-DFT approach. Since the corresponding dimensionless coupling constant $\lambda_{\mathrm{hb}}$ is proportional to $\left|g_{\mathrm{hb}}\right|^{2}$, one obtains that the bare $t-J$ coupling constant is almost three times larger than the LDADFT one:

$$
\lambda_{\mathrm{hb}}^{t-J} \approx 3 \lambda_{\mathrm{hb}}^{\mathrm{HF}}
$$

This example demonstrates clearly that the LDA-DFT method is inadequate for calculating the EPI coupling constant in HTSC cuprates.

Note that there is also a covalent contribution to EPI which comes from the change of the effective hopping $(t)$ in of the $t-J$ model $(77)$ and the exchange energy $(J)$ in the presence of atomic displacements:

$$
\begin{aligned}
\hat{H}_{e p}^{c o v}= & -\sum_{i, j, \sigma} \frac{\partial t_{i j}}{\partial\left(\mathbf{R}_{i}^{0}-\mathbf{R}_{j}^{0}\right)}\left(\widehat{\mathbf{u}}_{i}-\widehat{\mathbf{u}}_{j}\right) \hat{X}_{i}^{\sigma 0} \hat{X}_{j}^{0 \sigma} \\
& +\sum_{i, j,} \frac{\partial J_{i j}}{\partial\left(\mathbf{R}_{i}^{0}-\mathbf{R}_{j}^{0}\right)}\left(\widehat{\mathbf{u}}_{i}-\widehat{\mathbf{u}}_{j}\right) \mathbf{S}_{i} \cdot \mathbf{S}_{j} .
\end{aligned}
$$

Here, we will not go into details but only stress that since $\left|\epsilon_{i}^{0}\right| \gg\left|t_{i j}\right|$ then the covalent term in the effective $t-J$ model is much smaller than the ionic term-see more in $[6,10,11,169]$ and references therein-and in the following only the term $\hat{H}_{e p}^{\text {ion }}$ will be considered $[6,78-80,130]$.

\subsubsection{Controllable X-Method for the Quasiparticle Dynamics.} The minimal model Hamiltonian for strongly correlated holes with EPI (discussed above) is expressed via the Hubbard operators which obey "ugly" noncanonical commutation relations. The latter property is rather unpleasant for making a controllable theory in terms of Feynmann diagrams (for these "ugly" operators) and some other approaches are required. A possible way out is to express the Hubbard operators in terms of fermions and bosons (which must be confined) as, for instance, in the slave boson (SB) method. However, in real calculations which are based on some approximations the SB method is confronted with some subtle constraints whose fulfillments require very sophisticated mathematical treatment. Fortunately, there is a mathematically controllable approach for treating the problem directly with Hubbard operators and without using slave-boson (or fermion) techniques. This method-we call it the $X$ method-is based on the general Baym-Kadanoff technique which allows to treat the problem by the well-defined and controllable $1 / \mathrm{N}$ expansion for the Green's functions in terms of Hubbard operators. This approach is formulated in [214] while the important refinement of the method is done in [78-80, 130]. In the paramagnetic and homogeneous state (with finite doping) the Green's function $G_{\sigma_{1} \sigma_{2}}(1-2)$ is diagonal, that is, $G_{\sigma_{1} \sigma_{2}}(1-2)=\delta_{\sigma_{1} \sigma_{2}} G(1-2)$ where

$$
G(1-2)=-\left\langle T \hat{X}^{0 \sigma}(1) \hat{X}^{\sigma 0}(2)\right\rangle=g(1-2) Q,
$$

with the Hubbard spectral weight $Q=\left\langle\hat{X}^{00}\right\rangle+\left\langle\hat{X}^{\sigma \sigma}\right\rangle$. The function $g(1-2)$ plays the role of the quasiparticle Green's function-see more in $[6,78-80,130,179,180]$. It turns out that in order to have a controllable theory ( $1 / N$ expansion) one way is to increase the number of spin components from two to $N$ by changing the constraint (76) into the new one

$$
\hat{X}_{i}^{00}+\sum_{\sigma=1}^{N} \hat{X}_{i}^{\sigma \sigma}=\frac{N}{2} .
$$

In order to reach the convergence of physical quantities in the limit $N \rightarrow \infty$ the hopping and exchange energy are also rescaled, that is, $t_{i j}=t_{0, i j} / N$ and $J_{i j}=J_{0, i j} / N$. In order to eliminate possible misunderstandings we stress that in the case $N>2$ the constraint in (87) spoils some projection properties of the Hubbard operators. Fortunately, these (lost) projection properties are not used at all in the refined theory. As a result one obtains the functional integral equation for $G(1,2)$, thus allowing unambiguous mathematical and physical treatment of the problem. In [78-80, 130, 179, 180] it is developed a systematic $1 / \mathrm{N}$ expansion for the quasiparticle Green's function $g(1-2)\left(=g_{0}+g_{1} / N+\cdots\right), Q\left(=N q_{0}+\right.$ $\left.q_{1}+\cdots\right)$ ) (also for $G(1-2)$ ) and the self-energy. For large $N(\rightarrow \infty)$ the leading term is $G_{0}(1-2)=g_{0}(1-2) Q_{0}=O(N)$ with $g_{0}=\mathrm{O}(1)$ and $Q_{0}=\left\langle\hat{X}_{i}^{00}\right\rangle=N \delta / 2$. Here, $\delta$ is the concentration of the oxygen holes (that is, of the Zhang-Rice singlets) which is related to the chemical potential by the equation $1-\delta=2 \sum_{\mathbf{p}} n_{F}(\mathbf{p})$ with $n_{F}(\mathbf{p})=\left(e^{\epsilon_{0}(\mathbf{k})-\mu}+1\right)^{-1}$. The quasiparticle Green's function $g_{0}(\mathbf{k}, \omega)$ and the quasiparticle spectrum $\epsilon_{0}(\mathbf{k})$ in the leading order are given by

$$
\begin{gathered}
g_{0}(\mathbf{k}, \omega) \equiv \frac{G_{0}(\mathbf{k}, \omega)}{Q_{0}}=\frac{1}{\omega-\left[\epsilon_{0}(\mathbf{k})-\mu\right]} \\
\epsilon_{0}(\mathbf{k})=\epsilon_{c}-\delta \cdot t(\mathbf{k})-\sum_{\mathbf{p}} J_{0}(\mathbf{k}+\mathbf{p}) n_{F}(\mathbf{p}) .
\end{gathered}
$$

The level shift is $\epsilon_{c}=\epsilon^{0}+2 \sum_{\mathbf{p}} t(\mathbf{p}) n_{F}(\mathbf{p})$ and $t(\mathbf{p})$ is the Fourier transform of the hopping integral $t_{i j}$-see more in [6].

Let us summarize the main results of the X-method in leading $\mathrm{O}(1)$-order for the quasiparticle properties in the $t-J$ model $[6,78-80,130,179,180]$. (i) The Green's function $g_{0}(\mathbf{k}, \omega)$ describes the coherent motion of quasiparticles whose contribution to the total spectral weight of the Green's function $G_{0}(\mathbf{k}, \omega)$ is $Q_{0}=N \delta / 2$. The coherent motion of quasiparticles is described in leading order by $G_{0}(\mathbf{k}, \omega)=$ $Q_{0} g_{0}(\mathbf{k}, \omega)$ and the quasiparticle residuum $Q_{0}$ disappears in the undoped Mott insulating state $(\delta=0)$. This result is physically plausible since in the Mott insulating state the coherent motion of quasiparticles, which is responsible for finite conductivity, vanishes. (ii) The quasiparticle spectrum $\epsilon_{0}(\mathbf{k})$ plays the same role as the eigenvalues of the ideal bandstructure Hamiltonian $\hat{h}_{0}(\mathbf{x}, \mathbf{y})$ (it contains the excitation potential $V_{\mathrm{IBS}}(\mathbf{x}, \mathbf{y})$ which is due to high-energy processes of the Coulomb interaction). So, if we would consider $\epsilon_{t b}(\mathbf{k})=$ $-t(\mathbf{k})$ as the tight-binding parametrization of the LDA-DFT band-structure spectrum which takes int account only weak correlations (with the local potential $V_{x c}(x) \delta(x-y)$ ), then 
one can define a nonlocal excitation potential $V_{\mathrm{IBS}}^{t J}(\mathbf{x}, \mathbf{y})=$ $\tilde{V}_{\text {IBS }}^{t J}(\mathbf{x}, \mathbf{y})+V_{x c}(\mathbf{x}) \delta(\mathbf{x}-\mathbf{y})$ which mimics strong correlations in the $t-J$ model

$$
\tilde{V}_{\mathrm{IBS}}^{t J}(\mathbf{x}, \mathbf{y}) \approx V_{0} \delta(\mathbf{x}-\mathbf{y})+(1-\delta) t(\mathbf{x}-\mathbf{y})-\tilde{J}(\mathbf{x}-\mathbf{y})
$$

Here, $V_{0}=2 \sum_{\mathbf{p}} t(\mathbf{p}) n_{F}(\mathbf{p})$ and $t(\mathbf{x}-\mathbf{y})$ is the Fourier transform of $t(\mathbf{k})$ while $\widetilde{J}(\mathbf{x}-\mathbf{y})$ is the Fourier transform of the third term in (89). The relative excitation potential $\tilde{V}_{\text {IBS }}^{t J}(\mathbf{x}, \mathbf{y})$ is due to strong correlations (suppression of doubly occupancy on each lattice site) and as we will see below it is responsible for the short-range screening of EPI in such a way that the forward scattering peak appears in the effective EPI interaction-see discussion below. (iii) For the very low doping $\epsilon_{0}(\mathbf{k})$ is dominated by the exchange parameter if $J_{0}>\delta \cdot t_{0}$. However, in the case when $J_{0} \ll$ $\delta \cdot t_{0}$ there is a band narrowing by lowering the holedoping $\delta$, where the band width is proportional to the holeconcentration $\delta$, that is, $W_{b}=z \cdot \delta \cdot t_{0}$. (iv) The $\mathrm{O}(1)$-order quasiparticle Green's function $g_{0}(\mathbf{k}, \omega)$ and the quasiparticle spectrum $\epsilon_{0}(\mathbf{k})$ in the X-method have similar form as the spinon Green's function $g_{0, f}(\mathbf{k}, \omega)=-\left\langle T f_{\sigma} f_{\sigma}^{\dagger}\right\rangle_{\mathbf{k}, \omega}$ and the spinon energy $\epsilon_{s}(\mathbf{k})$ in the $S B$ method. However, in the $S B$ method there is a broken gauge symmetry in the metallic state (with $\delta \neq 0$ ) which is characterized by $\left\langle\hat{b}_{i}\right\rangle \neq 0$. This broken local gauge symmetry in the slave-boson method in $\mathrm{O}(1)$ order, which is due to the local decoupling of spinon and holon, is in fact forbidden by Elitzur's theorem. On the other side the local gauge invariance is not broken in the $\mathrm{X}$ method where Green's function $G_{0}(\mathbf{k}, \omega)$ describes motion of the composite object, that is, simultaneous creation of the hole and annihilation of the spin at a given lattice site, while in the $S B$ theory there is a spin-charge separation because of the broken symmetry $\left(\left\langle\hat{b}_{i}\right\rangle \neq 0\right)$. The assumption of the broken symmetry $\left\langle\hat{b}_{i}\right\rangle \neq 0$ gives qualitative satisfactory results for the quasiparticle energy for the case $N=\infty$ in $D>$ 2 dimensions. However, the analysis of response functions and of higher-order $1 / N$ corrections to the self-energies very delicate in the $S B$ theory and special techniques must be implemented in order to restore the gauge invariance of the theory. On the other side the $X$-method is intrinsically gauge invariant and free of spurious effects in all orders of the $1 / \mathrm{N}$ expansion. Therefore, one expects that these two methods may deliver different results in $\mathrm{O}(1)$ and higher order in response functions. This difference is already manifested in the calculation of EPI where the charge vertex in these two methods is peaked at different wave vectors $\mathbf{q}$, that is, at $\mathbf{q}=0$ in the X-method and $|\mathbf{q}| \neq 0$ in the SB methodsee Section 2.3.5. (v) In $[215,216]$ it is shown that in the superconducting state the anomalous self-energies (which are of $\mathrm{O}(1 / N)$-order in the $1 / N$ expansion) of the $X$ - and $S B$-methods differ substantially. As a consequence, the $S B$ method [217] predicts false superconductivity in the $t$ - $J$ model (for $J=0$ ) with large $T_{c}$ (due to the kinematical interaction), while the $X$-method gives extremely small $T_{c}(\approx$ $0)[215,216]$. So, although the two approaches yield some similar results in leading $\mathrm{O}(1)$-order they, are different at least in next to leading $\mathrm{O}(1 / N)$-order.

2.3.3. EPI Effective Potential in the $t$-J Model. The theory of EPI in the minimal $t$ - $J$ model based on the X-method predicts that the leading term in the EPI self-energy $\Sigma_{e p}$ is given by the expression $[6,78-80,130]$

$$
\Sigma_{e p}(1,2)=-V_{e p}(\overline{1}-\overline{2}) \gamma_{c}(1, \overline{3} ; \overline{1}) g_{0}(\overline{3}-\overline{4}) \gamma_{c}(\overline{4}, 2 ; \overline{2}),
$$

where the screened (by the dielectric constant) EPI potential

$$
V_{e p}(1-2)=\varepsilon_{e}^{-1}(1-\overline{1}) V_{e p}^{0}(\overline{1}-\overline{2}) \varepsilon_{e}^{-1}(\overline{2}-2)
$$

and $V_{e p}^{0}(1-2)=-\langle T \hat{\Phi}(1) \hat{\Phi}(2)\rangle$ is the "phonon" propagator which may also describe an anharmonic EPI. It is obvious that (91) is equivalent to (53) in spite the fact that the theory is formulated in terms of the Hubbard operators. The charge vertex $\gamma_{c}(1,2 ; 3)=-\delta g_{0}^{-1}(1,2) / \delta u_{\mathrm{eff}}(3)$ corresponds to the the renormalized vertex $\Gamma_{c, r}$ in (53) and it describes the screening by strong correlations. It depends on the relative excitation potential $\tilde{V}_{\text {IBS }}^{t J}(\mathbf{x}, \mathbf{y})$. The electronic dielectric function $\varepsilon_{e}(1-2)$ describes the screening of EPI by the long-range part of the Coulomb interaction. Note that in the harmonic approximation $\widehat{\Phi}(1)$ contains the bare EPI coupling constant $g_{e p}^{0}$ and lattice displacement $\hat{u}$, that is, $\widehat{\Phi} \sim g_{e p}^{0} \hat{u}$ - see more in [6]. (Note that in the above equations summation and integration over bar indices are understood.) The self-energy $\Sigma_{e p}(\mathbf{k}, \omega)$ due to EPI reads

$$
\Sigma_{e p}(\mathbf{k}, \omega)=\int_{0}^{\infty} d \nu\left\langle\alpha^{2} F\left(\mathbf{k}, \mathbf{k}^{\prime}, \nu\right)\right\rangle_{\mathbf{k}^{\prime}} R(\omega, \nu),
$$

with $R(\omega, \nu)=-2 \pi i\left(n_{\mathrm{B}}(\nu)+1 / 2\right)+\psi(1 / 2+i)-\psi(1 / 2-i(\nu+$ $\omega) / 2 \pi T)$ where $n_{\mathrm{B}}(\nu)$ is the Bose distribution function and $\psi$ is di-gamma function. The Eliashberg spectral function is given by

$$
\begin{aligned}
\alpha^{2} F\left(\mathbf{k}, \mathbf{k}^{\prime}, \omega\right)=N(0) \sum_{\nu} & \left|g_{\nu}\left(\mathbf{k}, \mathbf{k}-\mathbf{k}^{\prime}\right)\right|^{2} \\
& \times \delta\left(\omega-\omega_{\nu}\left(\mathbf{k}-\mathbf{k}^{\prime}\right)\right) \gamma_{c}^{2}\left(\mathbf{k}, \mathbf{k}-\mathbf{k}^{\prime}\right),
\end{aligned}
$$

where $g_{\nu}(\mathbf{k}, \mathbf{p})$ is the EPI coupling constant for the $v$ th mode, where the renormalization by long-range Coulomb interaction is included, that is, $g_{\nu}(\mathbf{k}, \mathbf{p})=g_{e p, v}^{0}(\mathbf{k}, \mathbf{p}) / \varepsilon_{e}(\mathbf{p})$. $\langle\cdots\rangle_{\mathbf{k}}$ denotes Fermi-surface average with respect to the momentum $\mathbf{k}$ and $N(0)$ is the density of states renormalized by strong correlations. The effect of strong correlations in the adiabatic limit is stipulated in the charge vertex function $\gamma_{c}\left(\mathbf{k}, \mathbf{k}-\mathbf{k}^{\prime}\right)$ which, as we will see in Section 2.3.4, changes the properties of $V_{e p}(\mathbf{q}, v)$ drastically compared to weakly correlated systems. In fact the charge vertex depends on frequency $\omega$ but in the adiabatic limit $\left(\omega_{\text {ph }} \ll W\right)$ and for $q v_{F}>\omega_{\text {ph }}$ it is practically frequency independent, that is, $\gamma_{c}^{(a d)}(\mathbf{k}, \mathbf{q}, \omega) \approx \gamma_{c}(\mathbf{k}, \mathbf{q}, \omega=0)$ where the latter is real quantity. For $J=0$ in the $t-t^{\prime}$ model the $1 / N$ expansion gives $N(0)=N_{0}(0) / q_{0}$ where $q_{0}=\delta / 2$. For $J \neq 0$ the density of states $N(0)$ does not diverge for $\delta \rightarrow 0$ where $N(0)(\sim$ $\left.1 / J_{0}\right)>N_{0}(0)$. The bare density of states $N_{0}(0)$ is calculated in 
absence of strong correlations, for instance, by the LDA-DFT method.

Depending on the symmetry of the superconducting order parameter $\Delta(\mathbf{k}, \omega)$ ( $s$ - and $d$-wave pairing) various projected averages (over the Fermi surface) of $\alpha^{2} F\left(\mathbf{k}, \mathbf{k}^{\prime}, \omega\right)$ enter the Eliashberg equations. Assuming that the superconducting order parameter transforms according to the representation $\Gamma_{i}$ of the point group $C_{4 v}$ of the square lattice (in the $\mathrm{CuO}_{2}$ planes) the appropriate symmetry-projected spectral function is given by

$$
\begin{aligned}
\alpha^{2} F_{i}\left(\widetilde{\mathbf{k}}, \widetilde{\mathbf{k}}^{\prime}, \omega\right)=\frac{N(0)}{8} \sum_{\nu, j} & \left|g_{\nu}\left(\widetilde{\mathbf{k}}, \tilde{\mathbf{k}}-T_{j} \widetilde{\mathbf{k}}^{\prime}\right)\right|^{2} \\
& \times \delta\left(\omega-\omega_{\nu}\left(\widetilde{\mathbf{k}}-T_{j} \widetilde{\mathbf{k}}^{\prime}\right)\right) \\
& \times \gamma_{c}^{2}\left(\widetilde{\mathbf{k}}, \tilde{\mathbf{k}}-T_{j} \widetilde{\mathbf{k}}^{\prime}\right) D_{i}(j)
\end{aligned}
$$

where $\widetilde{\mathbf{k}}$ and $\widetilde{\mathbf{k}}^{\prime}$ are momenta on the Fermi line in the irreducible Brillouin zone (1/8 of the total Brillouin zone). $T_{j}, j=1, \ldots, 8$ denotes the eight point-group transformations forming the symmetry group of the square lattice. This group has five irreducible representations which we distinguish by the label $i=1,2, \ldots, 5$. In the following we discuss the representations $i=1$ and $i=3$, which correspond to the $s$ - and $d$-wave symmetry of the full rotation group, respectively. $D_{i}(j)$ is the representation matrix of the $j$ th transformation for the representation $i$. Assuming that the superconducting order parameter $\Delta(\mathbf{k}, \omega)$ does not vary much in the irreducible Brillouin zone, one can average over $\tilde{\mathbf{k}}$ and $\tilde{\mathbf{k}}^{\prime}$ in the Brillouin zone. For each symmetry one obtains the corresponding pairing spectral function $\alpha^{2} F_{i}(\omega)$ :

$$
\alpha^{2} F_{i}(\omega)=\left\langle\left\langle\alpha^{2} F_{i}\left(\tilde{\mathbf{k}}, \tilde{\mathbf{k}}^{\prime}, \omega\right)\right\rangle_{\tilde{\mathbf{k}}}\right\rangle_{\tilde{\mathbf{k}}^{\prime}}
$$

which governs the transition temperature for the order parameter with the symmetry $\Gamma_{i}$. For instance, $\alpha^{2} F_{3}(\omega)$ is the pairing spectral function in the $d$-channel and it gives the coupling for $d$-wave superconductivity (the irreducible representation $\Gamma_{3}$-sometimes labelled as $B_{1 g}$ ). Performing similar calculations for the phonon-limited resistivity, one finds that the resistivity is related to the transport spectral function $\alpha^{2} F_{\mathrm{tr}}(\omega)$ :

$$
\alpha_{\mathrm{tr}}^{2} F(\omega)=\frac{\left\langle\left\langle\alpha^{2} F\left(\mathbf{k}, \mathbf{k}^{\prime}, \omega\right)\left[\mathbf{v}(\mathbf{k})-\mathbf{v}\left(\mathbf{k}^{\prime}\right)\right]^{2}\right\rangle\right\rangle_{\mathbf{k} \mathbf{k}^{\prime}}}{2\left\langle\left\langle\mathbf{v}^{2}(\mathbf{k})\right\rangle\right\rangle_{\mathbf{k} \mathbf{k}^{\prime}}} .
$$

The effect of strong correlations on EPI was discussed in [130] within the model where $g_{\nu}(\mathbf{k}, \mathbf{p})$ and the phonon frequencies $\omega_{\nu}\left(\tilde{\mathbf{k}}-\tilde{\mathbf{k}}^{\prime}\right)$ are weakly momentum dependent. In order to elucidate the main effect of strong correlations on EPI and $\alpha^{2} F_{i}(\omega)$ we consider the latter functions for a simple model with Einstein phonon, where these functions are proportional to the (so called) relative coupling constant $\Lambda_{i}$ :

$$
\Lambda_{i}=\frac{1}{8} \frac{N(0)}{N_{0}(0)} \sum_{j=1}^{8}\left\langle\left\langle\left|\gamma_{c}\left(\tilde{\mathbf{k}}, \tilde{\mathbf{k}}-T_{j} \tilde{\mathbf{k}}^{\prime}\right)\right|^{2}\right\rangle\right\rangle_{\tilde{\mathbf{k}} \tilde{\mathbf{k}}^{\prime}} D_{i}(j) .
$$

Similarly, the resistivity $\rho(T)\left(\sim \lambda_{\operatorname{tr}} \sim \Lambda_{\text {tr }}\right)$ is renormalized by the correlation effects where the transport coupling constant $\Lambda_{\text {tr }}$ is given by

$$
\Lambda_{\mathrm{tr}}=\frac{N(0)}{N_{0}(0)} \frac{\left\langle\left\langle\left|\gamma_{c}\left(\tilde{\mathbf{k}}, \tilde{\mathbf{k}}-T_{j} \tilde{\mathbf{k}}^{\prime}\right)\right|^{2}\left[\mathbf{v}(\mathbf{k})-\mathbf{v}\left(\mathbf{k}^{\prime}\right)\right]^{2}\right\rangle\right\rangle_{\mathbf{k k}^{\prime}}}{2\left\langle\left\langle\mathbf{v}^{2}(\mathbf{k})\right\rangle\right\rangle_{\mathbf{k k}^{\prime}}}
$$

As we see, all projected spectral functions $\alpha_{i}^{2} F(\omega)$ depend on the charge vertex function $\gamma_{c}(\mathbf{k}, \mathbf{q})$ which describes the screening (renormalization) of EPI due to strong correlations (suppression of doubly occupancy) [78-80, 130]. This important ingredient (which respects also the Ward identities) is a decisive step beyond the MFA renormalization of EPI in strongly correlated systems which was previously studied in connection with heavy fermions-see review in [218].

2.3.4. Charge Vertex and the EPI Coupling. The charge vertex function $\gamma_{c}(\mathbf{k}, \mathbf{q})$ (in the adiabatic approximation) has been calculated in $[78-80,130,179,180]$ in the framework of the $1 / N$ expansion in the $\mathrm{X}$-method-see also [6] - and here we discuss only the main results. Note that $\gamma_{c}(\mathbf{k}, \mathbf{q})$ renormalizes all charge fluctuation processes, such as the $E P I$ interaction, the long-range Coulomb interaction, the nonmagnetic impurity scattering, and so forth. In fact $\gamma_{c}(\mathbf{k}, \mathbf{q})$ describes specific screening due to the vanishing of doubly occupancy in strongly correlated systems. Note that the latter constraint is at present impossible to incorporate into the LDA-DFT band-structure calculations, thus making the latter method unreliable in highly correlated systems. In [78$80,130,179,180] \gamma_{c}(\mathbf{k}, \mathbf{q}, \omega)$ was calculated as a function of the model parameters $t, t^{\prime}, \delta, J$ in leading $\mathrm{O}(1)$ order of the $t-J$ model:

$$
\gamma_{c}(\mathbf{k}, q)=1-\sum_{\alpha=1}^{6} \sum_{\beta=1}^{6} F_{\alpha}(\mathbf{k})[\hat{1}+\hat{\chi}(q)]_{\alpha \beta}^{-1} \chi_{\beta 2}(q),
$$

where $\chi_{\alpha \beta}(q)=\sum_{p} G_{\alpha}(p, q) F_{\beta}(\mathbf{p}), F_{\alpha}(\mathbf{k})=[t(\mathbf{k}), 1$, $\left.2 J_{0} \cos k_{x}, 2 J_{0} \sin k_{x}, 2 J_{0} \cos k_{y}, 2 J_{0} \sin k_{y}\right]$, and $G_{\alpha}(p, q)$ $=\left[1, t(\mathbf{p}+\mathbf{q}), \cos p_{x}, \sin p_{x}, \cos p_{y}, \sin p_{y}\right] \Pi(p, q)$. Here, $\Pi(k, q)=-g(k) g(k+q)$ and $q=\left(\mathbf{q}, i q_{n}\right), q_{n}=2 \pi n T, p=$ $\left(\mathbf{p}, i p_{m}\right), p_{m}=\pi T(2 m+1)$. The physical meaning of the vertex function $\gamma_{c}(\mathbf{k}, q)$ is following: in the presence of an external (or internal) charge perturbation there is screening due to the change of the excitation potential $V_{\mathrm{IBS}}^{t J}(\mathbf{x}, \mathbf{y})$, that is, of the change of the bandwidth, as well as of the local chemical potential. The central result is that for momenta $\mathbf{k}$ lying at (and near) the Fermi surface the vertex function $\gamma_{c}(\mathbf{k}, \mathbf{q}, \omega=0)$ has very pronounced forward scattering peak (at $\mathbf{q}=0$ ) especially at very low doping concentration $\delta(\ll 1)$, while the backward scattering is substantially suppressed, as it is seen in Figure 35 where $\gamma_{c}\left(\mathbf{k}_{F}, \mathbf{q}, \omega=0\right)$ is shown. The peak at $q=0$ is very narrow at very small doping since its width $q_{c}$ is proportional to the doping $\delta$, that is, $q_{c} \sim \delta(\pi / a)$ where $a$ is the lattice constant. It is interesting that $\gamma_{c}(\mathbf{k}, q)$, as well as the dynamics of charge fluctuations, depend only weakly on the exchange energy $J$ and are mainly 


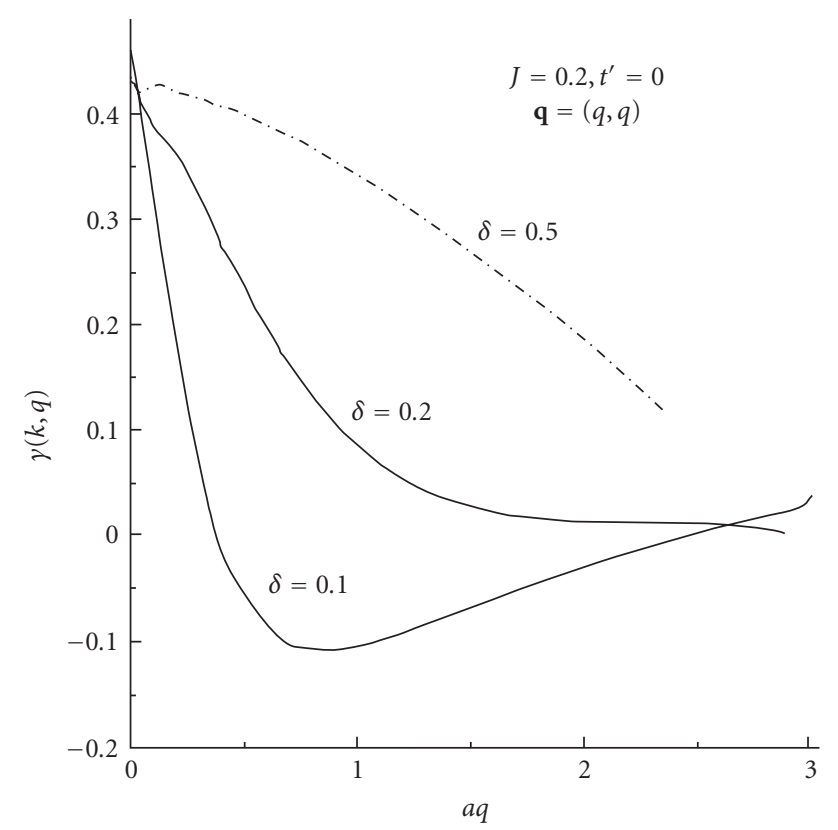

Figure 35: Adiabatic $(\omega=0)$ vertex function $\gamma\left(\mathbf{k}_{F}, \mathbf{q}\right)$ of the $t-J$ model as a function of the momentum $a q$ with $\mathbf{q}=(q, q)$ for three different doping levels $\delta$. From [130].

dominated by the constraint of having no doubly occupancy of sites, as it is shown in $[78-80,130,179,180]$.

The existence of the forward scattering peak in $\gamma_{c}(\mathbf{k}, \mathbf{q})$ at $q=0$ is confirmed by numerical calculations in the Hubbard model, which show that this peak is very pronounced at large $U$ [182]. This is important result since it proves that the $1 / \mathrm{N}$ expansion in the $X$-method is reliable method in studying charge fluctuation processes in strongly correlated systems. The strong suppression of $\gamma_{c}(\mathbf{k}, q)$ at large $q\left(\sim k_{F}\right)$ means that at small distances the charge fluctuations are strongly suppressed (correlated). Such a behavior of the vertex function means that a quasiparticle moving in the strongly correlated medium digs up a giant correlation hole with the radius $\xi_{\mathrm{ch}}\left(\sim \pi / q_{c}\right) \approx a / \delta$, where $a$ is the lattice constant. As a consequence of this effect the renormalized EPI becomes long ranged which is contrary to the weakly correlated systems where it is short ranged.

By knowing $\gamma_{c}(\mathbf{k}, q)$ one can calculate the relative coupling constants $\Lambda_{1} \equiv \Lambda_{s}, \Lambda_{3} \equiv \Lambda_{d}, \Lambda_{\text {tr }}$, and so forth. In the absence of correlations and for an isotropic band one has $\Lambda_{1}=\Lambda_{\text {tr }}=1, \Lambda_{i}=0$ for $i>1$. The averages in $\Lambda_{s}$, $\Lambda_{d}$, and $\Lambda_{\text {tr }}$ were performed numerically in [130] by using the realistic anisotropic band dispersion in the $t-t^{\prime}-J$ model and the results are shown in Figure 36. For convenience, the three curves are multiplied with a common factor so that $\Lambda_{s}$ approaches 1 in the empty-band limit $\delta \rightarrow 1$, when strong correlations are absent. Note that the superconducting critical temperature $T_{c}$ in the weak coupling limit and in the $i$ th channel scales like $T_{c}^{(i)} \sim \exp \left(-1 /\left(\lambda_{0} \Lambda_{i}-\mu_{i}^{*}\right)\right.$ where $\lambda_{0}$ is some effective coupling constant which depends on microscopic details. The parameter $\mu_{i}^{*}$ is the effective residual Coulomb repulsion in the $i$ th superconducting channel. We

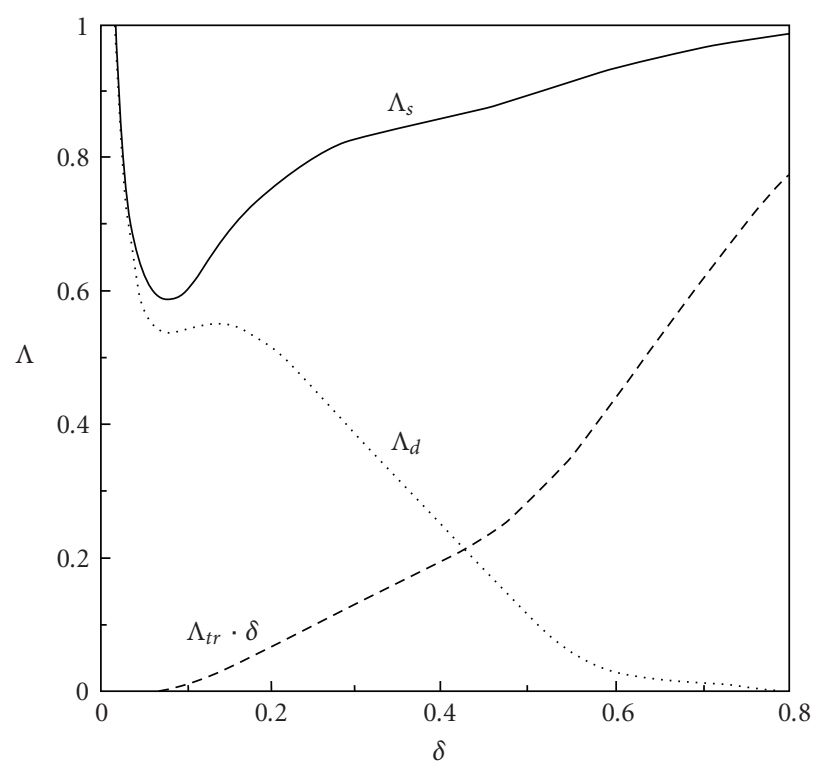

Figure 36: Normalized $s$-wave $\Lambda_{s}, d$-wave $\Lambda_{d}$, and transport $\Lambda_{\text {tr }} \cdot \delta$ coupling constants as a function of doping $\delta$ for $t^{\prime}=0$ and $J=0$. From $[179,180]$.

stress here several interesting results which come out from the above theory and which are partially presented in Figures 35 and 36.

(1) In principle the bare EPI coupling constant $g_{\lambda}^{0}(\mathbf{k}, \mathbf{q})$ depends on the quasiparticle momentum $\mathbf{k}$ and the transfer momentum q. In the $t-J$ model the EPI coupling is dominated by the ionic coupling $\hat{H}_{e p}^{\text {ion }}($ see $(80))$ and corresponding EPI depends only on the momentum transfer $\mathbf{q}$, that is, $g_{\lambda}^{0}(\mathbf{k}, \mathbf{q}) \approx g_{\lambda}^{0}(\mathbf{q})$ while for the much smaller covalent coupling $\hat{H}_{e p}^{\text {cov }}$ depends on both $\mathbf{k}$ and $\mathbf{q}[6,10,11]$. However, the EPI couplings for most phonon modes are renormalized by the charge vertex and since the latter is peaked at small momentum transfer $q=\left|\mathbf{k}-\mathbf{k}^{\prime}\right|$ then the maxima of the corresponding effective potentials are pushed toward smaller values of $q$. The further consequence of the vertex renormalization is that in the absence of strong correlations the bare EPI coupling $\left|g^{0}(\mathbf{k}, \mathbf{q})\right|^{2}$ for some phonon modes (which enters in the effective $t-J$ model) is detrimental for $d$-wave pairing; it can be less detrimental or even supports it in the presence of strong correlations (since the maximum is pushed toward smaller $q$ ). To illustrate this let us consider the in-plane oxygen breathing mode with the frequency $\omega_{\mathrm{br}}$ which is supposed to be important in HTSC cuprates. The bare coupling constant (squared) for this mode is approximately given by $\left|g_{\mathrm{br}}^{0}(\mathbf{k}, \mathbf{q})\right|^{2}=$ $\left|g_{\mathrm{br}}^{0}\right|^{2}\left[\sin ^{2}\left(q_{x} a / 2\right)+\sin ^{2}\left(q_{y} a / 2\right)\right]$ which reaches maximum for large $\mathbf{q}=(\pi / a, \pi / a)$. By extracting the component in the $d$ channel one has

$$
\left|g_{\mathrm{br}}^{0}\left(\mathbf{k}-\mathbf{k}^{\prime}\right)\right|^{2}=\left|g_{\mathrm{br}}^{0}\right|^{2}\left[1-\left(\frac{1}{4}\right) \psi_{d}(\mathbf{k}) \psi_{d}\left(\mathbf{k}^{\prime}\right)+\cdots\right]
$$


with

$$
\psi_{d}(\mathbf{k})=\cos k_{x} a-\cos k_{y} a .
$$

This gives the repulsive coupling constant $\lambda_{d}^{0}$ in the $d$ channel, that is,

$$
\lambda_{d}^{0}=\frac{2}{\omega_{\mathrm{br}}}\left\langle\psi_{d}(\mathbf{k})\left|g_{\mathrm{br}}^{0}\left(\mathbf{k}-\mathbf{k}^{\prime}\right)\right|^{2} \psi_{d}\left(\mathbf{k}^{\prime}\right)\right\rangle<0 .
$$

However, in the presence of strong correlations one expects that the effective coupling constant is given approximately by $\left|g_{\mathrm{br}}^{\text {eff }}\left(\mathbf{k}, \mathbf{k}-\mathbf{k}^{\prime}\right)\right| \approx\left|g_{\mathrm{br}}^{0}\left(\mathbf{k}-\mathbf{k}^{\prime}\right)\right|^{2} \gamma_{c}^{2}\left(\mathbf{k}_{F}, \mathbf{k}-\mathbf{k}^{\prime}\right)$ which is at small doping $\delta$ suppressed substantially at large $q$ since $\gamma_{c}^{2}$ starts to fall off drastically at $q \sim q_{c} \sim \delta(\pi / a)$. The latter property makes the effective coupling constant (in the $d$ channel) $\lambda_{d}^{\text {eff }}$ for these modes less negative or even positive (depending on the ratio $\xi_{\mathrm{ch}} / a \sim 1 / \delta$ ), that is, one has $\lambda_{d}^{\text {eff }}>$ $\lambda_{d}^{0}$. We stress again that this analysis is only qualitative (and semiquantitative) since it is based on the $t-J$ model while the better quantitative results are expected in the strongly correlated three-band Emery model with $U_{d} \gg t, \Delta_{p d}$ - see [6, Appendix D]. Unfortunately, these calculations are not finalized until now.

(2) In weakly correlated systems (or, e.g., in the emptyband limit $\delta \rightarrow 1$ ) the relative $d$-wave coupling constant $\Lambda_{d}$ is much smaller than the $s$-wave coupling constant $\Lambda_{s}$, that is, $\Lambda_{d} \ll \Lambda_{s}$ as it is seen in Figure 36. Furthermore, $\Lambda_{s}$ decreases with decreasing doping.

(3) It is indicative that independently on the value of $t^{\prime} \neq 0$ or $t^{\prime}=0$ the coupling constant $\Lambda_{s}$ and $\Lambda_{d}$ meet each other (note that $\Lambda_{s}>\Lambda_{d}$ for all $\delta$ ) at some small doping $\delta \approx 0.1-0.2$ where $\Lambda_{s} \approx \Lambda_{d}$. We would like to stress that such a unique situation (with $\Lambda_{s} \approx \Lambda_{d}$ ) was practically never realized in low-temperature and weakly correlated superconductors and in that respect the strong momentumdependent EPI in HTSC cuprates is an exclusive but very important phenomenon.

(4) By taking into account the residual Coulomb repulsion of quasiparticles then the $s$-wave superconductivity (which is governed by $\Lambda_{s}$ ) is suppressed, while the $d$ wave superconductivity (which is governed by $\Lambda_{d}$ ) stays almost unaffected, since $\mu_{s}^{*} \gg \mu_{d}^{*}$. In that case the $d$-wave superconductivity which is mainly governed by EPI becomes more stable than the $s$-wave one at sufficiently low doping $\delta$. This transition between $s$ - and $d$-wave superconductivity is triggered by electronic correlations because in the model calculations [78-80, 130] the bare EPI coupling is assumed to be momentum independent, that is, the bare coupling constant contains the $s$-wave symmetry only.

(5) The calculations of the charge vertex $\gamma_{c}$ are performed in the adiabatic limit, that is, for $\omega<\mathbf{q} \cdot \mathbf{v}_{F}(\mathbf{q})$ the frequency $\omega$ in $\gamma_{c}$ can be neglected. In the nonadiabatic regime, that is, for $\omega>\mathbf{q} \cdot \mathbf{v}_{F}(\mathbf{q})$, the function $\gamma_{c}^{2}\left(\mathbf{k}_{F}, \mathbf{q}, \omega\right)$ may be substantially larger compared to the adiabatic case because $\gamma_{c}\left(\mathbf{k}_{F}, \mathbf{q}, \omega\right)$ tends to the bare value 1 for $q=0$. This means that EPI for different phonons (with different energies $\omega$ ) is differently affected by strong correlations. For a given $\omega$ the EPI coupling to those phonons with momenta $q<q_{\omega}=\omega / v_{F}$ will be (relatively) enhanced since $\gamma_{c}\left(\mathbf{k}_{F}, \mathbf{q}, \omega\right) \approx 1$, while the coupling to those with $q>q_{\omega}=\omega / v_{F}$ will be substantially reduced due to the suppression of the backward scattering by strong correlations $[37,38]$. These results are a consequence of the Ward identities and generally hold in the LandauFermi liquid theory [219].

(6) The transport EPI coupling constant $\Lambda_{\text {tr }}$ is significantly reduced in the presence of strong correlations especially for low doping $(\delta \ll 1)$ where $\Lambda_{\operatorname{tr}}<\Lambda / 3$. This result is physically plausible since the resistivity is dominated by the backward scattering processes (large $q \sim k_{F}$ ) which are suppressed by strong correlations-the suppression of $\gamma_{c}\left(\mathbf{k}_{F}, \mathbf{q}, \omega\right)$ at large $q$.

The theory based on the forward scattering peak in EPI is a good candidate to explain the linear temperature behavior of the resistivity down to very small temperatures $T\left(\sim \Theta_{D} / 30\right) \approx 10 \mathrm{~K}$ in some cuprates with low $T_{c}(\approx 10 \mathrm{~K})$ $[6,128,129]$. One physically rather plausible model, which is based on the forward scattering peak in EPI, is elaborated in [128]. It takes into account (i) the quasiparticle scattering on acoustic (a) and on optic (o) phonons, (ii) the extended van Hove singularity in the quasiparticle density of states $N(\xi)$ which in some cuprates is very near the Fermi surface, and (iii) the umklapp and "undulation" (due to the flat regions at the Fermi surface) processes with $\mathbf{v}_{\mathbf{k}^{\prime}} \cong-\mathbf{v}_{\mathbf{k}}-$ this condition can partly increase the EPI coupling. The transport Eliashberg function $\alpha_{\mathrm{tr}}^{2} F(\omega)$ is calculated similarly to (97) by using the definition of $\alpha^{2} F\left(\mathbf{k}, \mathbf{k}^{\prime}, \omega\right)$ in (95) with the renormalized coupling constant $g_{\nu}^{(r)}\left(\mathbf{k}-\mathbf{k}^{\prime}\right)=g_{\nu}(\mathbf{k}-$ $\left.\mathbf{k}^{\prime}\right) \gamma_{c}\left(\mathbf{k}-\mathbf{k}^{\prime}\right)$ of the $v=a, o$ mode, respectively. In [128] it is assumed a phenomenological form for the forward scattering peak in $\gamma_{c}\left(\mathbf{k}-\mathbf{k}^{\prime}\right)$ with the cutoff $q_{c} \ll k_{F}$ (and which mimics the exact results from $[78-80,130,179,180])$. Since the scattering of the quasiparticles on phonons (with the sound velocity $v_{s}$ ) is limited to small- $q$ transfer processes (with $q<q_{c}$ ), then the maximal energy of the acoustic branch is not the Debye energy $\Theta_{D}\left(\approx k_{F} v_{s}\right)$ but the effective Debye energy $\Theta_{A}\left(\approx q_{c} v_{s}\right) \ll \Theta_{D}$. In the case of Bi2201 in [128] it is taken (from the numerical results in $[78-80,130,179,180]$ ) that $q_{c} \approx k_{F} / 10$ which gives $\Theta_{A} \approx(30-50) K$. As a result the calculated $\alpha_{\mathrm{tr}}^{2} F(\omega)$ gives that $\rho_{a b}(T) \sim T$ down to very low $T\left(\sim 0.2 \Theta_{A}\right) \approx 10 \mathrm{~K}$. The slope $\left(d \rho_{a b} / d T\right)$ is governed by the effective EPI coupling constant for acoustic phonons. In systems with the extended van Hove singularity (in $N(\xi)$ ) near the Fermi surface, which is the case in Bi-2201, the effective coupling constant for acoustic phonons can be sufficiently large to give experimental values for the slope $\left(d \rho_{a b} / d T\right) \sim(0.5-1) \mu \Omega \mathrm{cm} / \mathrm{K}$-for details see [128]. This physical picture is applicable also to cuprates near and at the optimal doping but since in these systems $T_{c}$ is large the linearity of $\rho_{a b}(T)$ down to very low $T$ is "screened" by the appearance of superconductivity.

(7) The width of the forward scattering peak in $\gamma_{c}\left(\mathbf{k}_{F}, \mathbf{q}\right)$ is very narrow in underdoped cuprates — with the width $q_{c} \sim$ $\delta(\pi / a)$ — which may have further interesting consequences. For instance, HTSC cuprates are characterized not only by strong correlations but also by the relatively small Fermi energy $E_{F}$, which is in underdoped systems not much larger than the characteristic (maximal) phonon frequency $\omega_{\mathrm{ph}}^{\max }$, 
that is, $E_{F} \simeq 0.1-0.3 \mathrm{eV}, \omega_{\mathrm{ph}}^{\max } \simeq 80 \mathrm{meV}$. Due to the appreciable magnitude of $\omega_{D} / E_{F}$ it is necessary to correct the Migdal-Eliashberg theory by the non-Migdal vertex corrections due to the EPI. It is well known that these vertex corrections lower $T_{c}$ in systems with the isotropic EPI. However, the non-Migdal vertex corrections in systems with the forward scattering peak in the EPI coupling with the cutoff $q_{c} \ll k_{F}$ may increase $T_{c}$ which can be appreciable. The corresponding calculations [220, 221] give two interesting results: (i) there is an appreciable increase of $T_{c}$ by lowering $Q_{c}=q_{c} / 2 k_{F}$, for instance, $T_{c}\left(Q_{c}=0.1\right) \approx$ $4 T_{c}\left(Q_{c}=1\right)$; (ii) even small values of $\lambda_{e p}<1$ can give large $T_{c}$. The latter results open a new possibility in reaching high $T_{c}$ in systems with appreciable ratio $\omega_{D} / E_{F}$ and with the forward scattering peak in EPI. The difference between the Migdal-Eliashberg and the non-Migdal theory can be explained qualitatively in the framework of an approximative McMillan formula for $T_{c}$ (for not too large $\lambda$ ) which reads $T_{c} \approx\langle\omega\rangle e^{-1 /\left(\tilde{\lambda}-\mu^{*}\right)}$. The Migdal-Eliashberg theory predicts

$$
\tilde{\lambda}^{(\mathrm{ME})} \approx \frac{\lambda}{1+\lambda},
$$

while the non-Migdal theory [220, 221] gives

$$
\tilde{\lambda}^{(\mathrm{n}-\mathrm{ME})} \approx \lambda(1+\lambda) .
$$

For instance, $T_{c} \sim 100 \mathrm{~K}$ in HTSC oxides can be explained by the Migdal-Eliashberg theory for $\lambda^{(\mathrm{ME})} \sim 2$, while in the nonMigdal theory much smaller coupling constant is needed, that is, $\lambda^{(\mathrm{n}-\mathrm{ME})} \sim 0.5$.

(8) The existence of the forward scattering peak in EPI can in a plausible way explain the ARPES puzzle that the antinodal kink is shifted by the maximal superconducting gap $\Delta_{\max }$ while the nodal kink is unshifted. The reason is (as explained in Section 1.3.3) that due to strong correlations the EPI spectral function $\alpha^{2} F\left(\mathbf{k}, \mathbf{k}^{\prime}, \Omega\right) \approx \alpha^{2} F\left(\varphi-\varphi^{\prime}, \Omega\right)$ is strongly peaked at $\varphi-\varphi^{\prime}=0$ [151].

(9) The scattering potential on nonmagnetic impurities is renormalized by strong correlations giving also the forward scattering peak in the impurity scattering potential (amplitude) [155]. The latter effect gives large $d$-wave channel in the renormalized impurity potential, which is the reason that $d$-wave pairing in HTSC cuprates is robust in the presence of nonmagnetic impurities (and defects) $[6,155]$.

2.3.5. EPI and Strong Correlations-Other Methods. The calculations of the static (adiabatic) charge-vertex $\gamma_{c}\left(\mathbf{k}_{F}, \mathbf{q}\right.$ ) in the X-method are done for the case $U=\infty[78-80,130$, $179,180]$ where it is found that it is peaked at $q=0$ the forward scattering peak (FSP). This result is confirmed by the numerical Monte Carlo calculations for the finite- $U$ Hubbard model [182], where it is found that FSP exists for all $U$, but it is especially pronounced in the limit $t \ll U$. These results are additionally confirmed in the calculations [183] within the four slave-boson method of Kotliar-Rückenstein where $\gamma_{c}\left(\mathbf{k}_{F}, \mathbf{q}\right)$ is again peaked at $\mathbf{q}=0$ and the peak is also pronounced at $t \ll U$.

There are several calculations of the charge vertex in the one slave-boson method [219, 222-224] which is invented to study the limit $U \rightarrow \infty$. It is interesting to compare the results for the charge vertex in the X-method [78-80, 130, $179,180]$ and in the one slave-boson theory [222] which are calculated in $O\left((1 / N)^{0}\right)$ order. For instance, for $J=0$ one has

$$
\begin{gathered}
\gamma_{c}^{(X)}(\mathbf{k}, \mathbf{q})=\frac{1+b(\mathbf{q})-a(\mathbf{q}) t(\mathbf{k})}{[1+b(\mathbf{q})]^{2}-a(\mathbf{q}) c(\mathbf{q})}, \\
\gamma_{c}^{(\mathrm{SB})}(\mathbf{k}, \mathbf{q})=\frac{1+b(\mathbf{q})-a(\mathbf{q})[t(\mathbf{k})+t(\mathbf{k}+\mathbf{q})] / 2}{[1+b(\mathbf{q})]^{2}-a(\mathbf{q}) c(\mathbf{q})} .
\end{gathered}
$$

The explicit expressions for the "bare" susceptibilities $a(\mathbf{q}), b(\mathbf{q})$, and $c(\mathbf{q})$ can be found in [78-80, 130]. It is obvious from (106) that $\gamma_{c}^{(X)}(\mathbf{k}, \mathbf{q}=0)=\gamma_{c}^{(\mathrm{SB})}(\mathbf{k}, \mathbf{q}=0)$ but the calculations give that $\max \left\{\gamma_{c}^{(X)}(\mathbf{k}, \mathbf{q})\right\}$ is for $\mathbf{q}=$ 0 , while $\max \left\{\gamma_{c}^{(\mathrm{SB})}(\mathbf{k}, \mathbf{q})\right\}$ is for $|\mathbf{q}| \neq 0$ [181]. So, the SB vertex is peaked at finite $q$ which is in contradiction with the numerical Monte Carlo results for the Hubbard model [182] and with the four slave-boson theory [183]. The reason for the discrepancy of the one slave-boson (SB) in studying EPI with the numerical results and the $\mathrm{X}$-method is not quite clear and might be due to the symmetry breaking of the local gauge invariance in leading order of the SB theory.

2.4. Summary of Section 2. The experimental results in HTSC cuprates which are exposed in Section 1 imply that the EPI coupling constant is large and in order to be conform with $d$-wave pairing this interaction must be very nonlocal (long range), that is, weakly screened and peaked at small transfer momenta. In absence of quantitative calculations in the framework of the ab initio microscopic many-body theory the effects of strong correlations on EPI are studied within the minimal $t-J$ model where this pronounced nonlocality is due to two main reasons: (1) strong electronic correlations and (2) the combined metallicionic layered structure in these materials. In case (1) the pronounced nonlocality of EPI, which is found in the $t-J$ model system, is due to the suppression of doubly occupancy at the $\mathrm{Cu}$ lattice sites in the $\mathrm{CuO}_{2}$ planes, which drastically weakens the screening effect in these systems. The pronounced nonlocality and suppression of the screening are mathematically expressed by the charge vertex function $\gamma_{c}\left(\mathbf{k}_{F}, \mathbf{q}, \omega\right)$ which multiplies the bare EPI matrix element. The vertex function is peaked at $q=0$ and strongly suppressed at large $q$, especially for low (oxygen) holedoping $\delta \ll 1$ near the Mott-Hubbard transition. Such a structure of $\gamma_{c}$ gives that the $d$-wave and $s$-wave coupling constants are of the same order of magnitude around and below some optimal doping $\delta_{\mathrm{op}} \approx 0.1$, that is, $\lambda_{d} \approx \lambda_{s}$. This is very peculiar situation never met before. Since the residual effective (low-energy) Coulomb interaction is much smaller in the $d$-channel than in the $s$-channel, that is, $\mu_{s}^{*} \gg \mu_{d}^{*}$ (with the possibility that $\mu_{d}^{*}<0$ ), then the critical temperature for $d$-wave pairing is much larger than for the $s$-wave one, that is, $T_{c}^{(d)} \gg T_{c}^{(s)}$. Since all charge fluctuation processes are modified by strong correlations, then the quasiparticle scattering on nonmagnetic impurities is also drastically changed; the pair-breaking effect on $d$-wave 
pairing is drastically reduced. This nonlocal effect, which is not discussed here- see more in [6] and references thereinis one of the main reasons for the robustness of $d$-wave pairing in HTSC oxides in the presence of nonmagnetic impurities and numerous local defects. The development of the forward scattering peak in $\gamma_{c}\left(\mathbf{k}_{F}, \mathbf{q}\right)$ and suppression at large $q\left(\gg q_{c}=\delta(\pi / a)\right)$ give rise to the suppression of the transport coupling constant $\lambda_{\text {tr }}$ making it much smaller than the self-energy coupling constant $\lambda$, that is, one has $\lambda_{\text {tr }} \approx \lambda / 3$ near the optimal doping $\delta=0.1-0.2$. Thus the behavior of the vertex function and the dominance of EPI in the quasiparticle scattering resolve the experimental puzzle that the transport and the self-energy coupling constant take very different values, $\lambda_{\text {tr }, e p} \ll \lambda_{e p}$. Note that this is not the case with the SFI mechanism which is dominant at large $\mathbf{q} \approx \mathbf{Q}=(\pi, \pi)$ thus giving $\lambda_{\mathrm{tr}, \mathrm{sf}} \approx \lambda_{\mathrm{sf}}$. This result means that if in the SFI mechanism one fits the temperature-dependent resistivity (governed by $\lambda_{\mathrm{tr}, \mathrm{sf}}$ ) then one obtains very low $T_{c}$.

We stress that the strength of the EPI coupling constants $\lambda_{e p}, \lambda_{e p, d}$ is at present impossible to calculate since it is difficult to incorporate strong correlations and numerous structural effects in a tractable microscopic theory.

2.5. Discussions and Conclusions. Numerous experimental results related to tunnelling, optics, ARPES, inelastic neutron, and X-ray scattering measurements in HTSC cuprates at and near the optimal doping give evidence for strong electron-phonon interaction (EPI) with the coupling constant $1<\lambda_{e p}<3.5$. The tunnelling measurements furnish evidence for strong EPI which give that the peaks in the bosonic spectral function $\alpha^{2} F(\omega)$ coincide well with the peaks in the phonon density of states $F_{\mathrm{ph}}(\omega)$. The tunnelling spectra show that almost all phonons contribute to $T_{c}$ and that no particular phonon mode can be singled out in the spectral function $\alpha^{2} F(\omega)$ as being the only one which dominates in pairing mechanism. In light of these results the small oxygen isotope effect in optimally doped systems can be partly due to this effect, thus not disqualifying the important role of EPI in pairing mechanism. The compatibility of the strong EPI with $d$-wave pairing implies an important constraint on the EPI pairing potential - it must be nonlocal, that is, peaked at small transfer momenta. The latter is due to (a) strong electronic correlations and (b) the combined metallicionic structure of these materials. If the EPI scattering is the main player in pairing in HTSC cuprates, then this nonlocality implies that at and below some optimal doping $\left(\delta_{\mathrm{op}} \sim 0.1\right)$ the magnitude of the EPI coupling constants in $d$-wave and $s$-wave channel must be of the same order, that is, $\lambda_{e p, d} \approx \lambda_{e p, s}$. This result in conjunction with the fact that the residual effective Coulomb coupling in $d$-wave channel is much smaller than in the $s$-wave one, that is, $\mu_{s}^{*} \gg \mu_{d}^{*}$ (with the possibility that $\mu_{d}^{*}<0$ ) gives that the critical temperature for $d$-wave pairing is much larger than for $s$-wave pairing.

The numerous tunnelling, ARPES, optics, and magnetic neutron scattering measurements give sufficient evidence that the spin-fluctuation interaction (SFI) plays a secondary role in pairing in HTSC cuprates. Especially important evidence for the smallness of SFI (in pairing) comes from the magnetic neutron scattering measurements which show that by varying doping slightly around the optimal one there is a huge reconstruction of the SFI spectral function $\operatorname{Im} \chi(\mathbf{q}, \omega)$ (imaginary part of the spin susceptibility) for $\mathbf{q} \approx \mathbf{Q}$, while there is very small change in the critical temperature $T_{c}$. These experimental results imply important constraints on the pairing scenario for systems at and near optimal doping: (1) the strength of the $d$-wave pairing potential is provided by EPI (i.e., one has $\lambda_{e p, d} \approx \lambda_{e p, s}$ ) while the role of the residual Coulomb interaction and SFI, together, is to trigger $d$-wave pairing; (2) the Migdal-Eliashberg theory, but with the pronounced momentum dependent of EPI, is a rather good starting theory.

The ab initio microscopic theory of pairing in HTSC cuprates fails at present to calculate $T_{c}$ and to predict the magnitude of the $d$-wave order parameter. From that point of view it is hard to expect a significant improvement of this situation at least in the near future. However, the studies of some minimal (toy) models, such as the singleband $t-J$ model, allow us to understand part of the physics in cuprates on a qualitative and in some cases even on a semiquantitative level. In that respect the encouraging results come from the theoretical studies of the EPI scattering in the $t-J$ model by using controllable mathematical methods in the X-method formulated in terms of the Hubbard operators $[78-80,130,179,180]$. This theory predicts dressing of quasiparticles by strong correlations which dig up a largescale correlation hole of the size $\xi_{\mathrm{ch}} \sim a / \delta$ for $\delta \ll 1$. These quasiparticles respond to lattice vibrations in such a way to produce an effective long-range electron interaction (due to EPI), that is, the effective pairing potential $V_{\text {eff }}(\mathbf{q}, \omega)$ is peaked at small transfer momenta $q$ - the forward scattering peak. This theory (of the toy model) is conform with the experimental scenario by predicting the following results: (i) the EPI coupling constants in $d$-wave and $s$-wave channels are of the same order, that is, $\lambda_{e p, d} \approx \lambda_{e p, s}$, at some optimal doping $\delta_{\mathrm{op}} \sim 0.1$; (ii) the transport coupling is much smaller than the pairing one, that is, $\lambda_{\operatorname{tr}} \approx \lambda / 3$; (iii) due to strong correlations there is forward scattering peak in the potential for scattering on nonmagnetic impurities, thus making $d$-wave pairing robust in materials with a lot of defects and impurities. Applied to HTSC superconductors at and near the optimal doping, this theory is a realization of the Migdal-Eliashberg theory but with strongly momentum dependent EPI coupling, which is conform with the proposed experimental pairing scenario. This scenario which is also realized in the $t-J$ toy model may be useful in making a (phenomenological) theory of pairing in cuprates. However, all present theories are confronted with the unsolved and challenging task - the calculation of $T_{c}$. From that point of view we do not have at present a proper microscopic theory of pairing in HTSC cuprates.

\section{Appendix}

\section{A. Spectral Functions}

A.1. Spectral Functions $\alpha^{2} F\left(k, k^{\prime}, \omega\right)$ and $\alpha^{2} F(\omega)$. The quasiparticle bosonic (Eliashberg) spectral function $\alpha^{2} F\left(\mathbf{k}, \mathbf{k}^{\prime}, \omega\right)$ and its Fermi surface average $\alpha^{2} F(\omega)=\left\langle\left\langle\alpha^{2} F\left(\mathbf{k}, \mathbf{k}^{\prime}, \omega\right)\right\rangle\right\rangle_{\mathbf{k}, \mathbf{k}^{\prime}}$ 
enter the quasiparticle self-energy $\Sigma(\mathbf{k}, \omega)$, while the transport spectral function $\alpha^{2} F_{\mathrm{tr}}(\omega)$ enters the transport selfenergy $\Sigma_{\operatorname{tr}}(\mathbf{k}, \omega)$ and dynamical conductivity $\sigma(\omega)$. Since the Migdal-Eliashberg theory for EPI is well defined, we define the spectral functions for this case and the generalization to other electron-boson interaction is straightforward. In the superconducting state Matsubara Green's functions $\hat{G}\left(\mathbf{k}, \omega_{n}\right)$ and $\hat{\Sigma}\left(\mathbf{k}, \omega_{n}\right)$ are $2 \times 2$ matrices with the diagonal elements $G_{11} \equiv G\left(\mathbf{k}, \omega_{n}\right), \Sigma_{11} \equiv \Sigma\left(\mathbf{k}, \omega_{n}\right)$ and the off-diagonal elements $G_{12} \equiv F\left(\mathbf{k}, \omega_{n}\right), \Sigma_{12} \equiv \Phi\left(\mathbf{k}, \omega_{n}\right)$ which describe superconducting pairing. By defining $i \omega_{n}\left[1-Z\left(\mathbf{k}, \omega_{n}\right)\right]=$ $\left[\Sigma\left(\mathbf{k}, \omega_{n}\right)-\Sigma\left(\mathbf{k},-\omega_{n}\right)\right] / 2$ and $\chi\left(\mathbf{k}, \omega_{n}\right)=\left[\Sigma\left(\mathbf{k}, \omega_{n}\right)+\right.$ $\left.\Sigma\left(\mathbf{k},-\omega_{n}\right)\right] / 2$, the Eliashberg functions for EPI in the presence of the Coulomb interaction (in the singlet pairing channel) read [70, 225-227]

$$
\begin{aligned}
& Z\left(\mathbf{k}, \omega_{n}\right)=1+\frac{T}{N} \sum_{\mathbf{p}, m} \frac{\lambda_{\mathbf{k p}}^{Z}\left(\omega_{n m}^{-}\right) \omega_{m}}{N(\mu) \omega_{n}} \frac{Z\left(\mathbf{p}, \omega_{m}\right)}{D\left(\mathbf{p}, \omega_{m}\right)}, \\
& \chi\left(\mathbf{k}, \omega_{n}\right)=-\frac{T}{N} \sum_{\mathbf{p}, m} \frac{\lambda_{\mathbf{k p}}^{Z}\left(\omega_{n m}^{-}\right)}{N(\mu)} \frac{\epsilon(\mathbf{p})-\mu+\chi\left(\mathbf{p}, \omega_{m}\right)}{D\left(\mathbf{p}, \omega_{m}\right)}, \\
& \Phi\left(\mathbf{k}, \omega_{n}\right)=\frac{T}{N} \sum_{\mathbf{p}, m}\left[\frac{\lambda_{\mathbf{k p}}^{\Delta}\left(\omega_{n m}^{-}\right)}{N(\mu)}-V_{\mathbf{k p}}\right] \frac{\Phi\left(\mathbf{p}, \omega_{m}\right)}{D\left(\mathbf{p}, \omega_{m}\right)},
\end{aligned}
$$

where $\omega_{n m}^{-} \equiv \omega_{n}-\omega_{m}, \omega_{n}=\pi T(2 n+1), \Phi\left(\mathbf{k}, \omega_{n}\right) \equiv$ $Z\left(\mathbf{k}, \omega_{n}\right) \Delta\left(\mathbf{k}, \omega_{n}\right), D=\omega_{m}^{2} Z^{2}+(\epsilon-\mu+\chi)^{2}+\Phi^{2}$, and $N(\mu)$ is the density of states at the Fermi surface. (In studying some problems, such as optics, it is useful to define the renormalized frequency $i \tilde{\omega}_{n}\left(i \omega_{n}\right)\left(\equiv i \omega_{n} Z\left(\omega_{n}\right)\right)=\omega_{n}-\Sigma\left(\omega_{n}\right)$ or its analytical continuation $\widetilde{\omega}(\omega)=Z(\omega) \omega=\omega-\Sigma(\omega))$. These equations are supplemented with the electron number equation $n(\mu)$ ( $\mu$ is the chemical potential):

$$
\begin{aligned}
n(\mu) & =\frac{2 T}{N} \sum_{\mathbf{p}, m} G\left(\mathbf{p}, \omega_{m}\right) e^{i \omega_{m} 0^{+}} \\
& =1-\frac{2 T}{N} \sum_{\mathbf{p}, m} \frac{\epsilon(\mathbf{p})-\mu+\chi\left(\mathbf{p}, \omega_{m}\right)}{D\left(\mathbf{p}, \omega_{m}\right)} .
\end{aligned}
$$

Note that in the case of EPI one has $\lambda_{\mathbf{k p}}^{\Delta}\left(\nu_{n}\right)=\lambda_{\mathbf{k p}}^{Z}\left(\nu_{n}\right)(\equiv$ $\lambda_{\mathbf{k p}}\left(\nu_{n}\right)$ ) (with $\nu_{n}=\pi T n$ ) where $\lambda_{\mathbf{k p}}\left(\nu_{n}\right)$ is defined by

$$
\begin{array}{r}
\lambda_{\mathbf{k p}}\left(\nu_{n}\right)=2 \int_{0}^{\infty} d \nu \frac{\nu}{\nu^{2}+\nu_{n}^{2}} \alpha_{\mathbf{k p}}^{2} F(\nu), \\
\alpha_{\mathbf{k} \mathbf{p}}^{2} F(\nu)=N(\mu) \sum_{\kappa}\left|g_{\kappa, \mathbf{k p}}^{\text {ren }}\right|^{2} B_{\kappa}(\mathbf{k}-\mathbf{p}, \nu),
\end{array}
$$

where $B_{\kappa}(\mathbf{k}-\mathbf{p} ; \nu)$ is the phonon spectral function of the $\kappa$ th phonon mode related to the phonon propagator

$$
D_{\kappa}\left(\mathbf{q}, i v_{n}\right)=-\int_{0}^{\infty} d v \frac{v}{v^{2}+v_{n}^{2}} B_{\kappa}(\mathbf{q}, v)
$$

However, very often it is measured the generalized phonon density of states $\operatorname{GPDS}(\omega)(\equiv G(\omega))$ (see Section 1.3.4) defined by $G(\omega)=\sum_{i}\left(\sigma_{i} / M_{i}\right) F_{i}(\omega) / \sum_{i}\left(\sigma_{i} / M_{i}\right)$. Here, $\sigma_{i}$ and $M_{i}$ are the cross-section and the mass of the $i$ th nucleus and
$F_{i}\left(\omega=(1 / N) \sum_{q}\left|\varepsilon_{q}^{i}\right|^{2} \delta\left(\omega-\omega_{q}\right)\right.$ is the amplitude-weighted density of states.

The renormalized coupling constant $g_{\kappa, \mathbf{k p}}^{\text {ren }}\left(\approx g_{\kappa, \mathbf{k p}}^{0} \gamma \varepsilon_{e}^{-1}\right)$ in (A.4) comprises the screening effect due to long-range Coulomb interaction $\left(\sim \mathcal{E}_{e}^{-1}\right.$-the inverse electronic dielectric function) and short-range strong correlations $(\sim \gamma-$ the vertex function) - see more in Section 2. Usually in the case of low-temperature superconductors (LTS) with $s$-wave pairing the anisotropy is rather small (or in the presence of impurities it is averaged out) which allows an averaging of the Eliashberg equations [70, 225-227]:

$$
\begin{gathered}
Z\left(\omega_{n}\right)=1+\frac{\pi T}{\omega_{n}} \sum_{m} \frac{\lambda\left(\omega_{n m}^{-}\right) \omega_{m}}{\sqrt{\omega_{m}^{2}+\Delta^{2}\left(\omega_{m}\right)}}, \\
Z\left(\omega_{n}\right) \Delta\left(\omega_{n}\right)=\pi T \sum_{m}\left[\lambda\left(\omega_{n m}^{-}\right)-\mu\left(\omega_{c}\right) \theta\left(\omega_{c}-\left|\omega_{m}\right|\right)\right] \\
\times \frac{\Delta\left(\omega_{m}\right)}{\sqrt{\omega_{m}^{2}+\Delta^{2}\left(\omega_{m}\right)}}, \\
\lambda\left(\omega_{n m}^{-}\right)=2 \int_{0}^{\infty} d \nu \frac{\nu}{\nu^{2}+\left(\omega_{n m}^{-}\right)^{2}} \alpha^{2} F(\nu),
\end{gathered}
$$

where $\omega_{n m}^{-}=\omega_{n}-\omega_{m}, \alpha^{2} F(\omega)=\left\langle\left\langle\alpha^{2} F\left(\mathbf{k}, \mathbf{k}^{\prime}, \omega\right)\right\rangle\right\rangle_{\mathbf{k}, \mathbf{k}^{\prime}}$, and $\langle\langle\cdots\rangle\rangle_{\mathbf{k}, \mathbf{k}^{\prime}}$ is the average over the Fermi surface. The above equations can be written on the real axis by the analytical continuation $i \omega_{m} \rightarrow \omega+i \delta$ where the gap function is complex, that is, $\Delta(\omega)=\Delta_{R}(\omega)+i \Delta_{I}(\omega)$. The solution for $\Delta(\omega)$ allows the calculation of the current-voltage characteristic $I(V)$ and tunnelling conductance $G_{\mathrm{NS}}(V)=$ $d I_{\mathrm{NS}} / d V$ in the superconducting state of the NIS tunnelling junction where $I_{\mathrm{NS}}(V)$ is given by

$$
\begin{gathered}
I_{\mathrm{NS}}(V)=2 e \sum_{\mathbf{k}, \mathbf{p}}\left|T_{\mathbf{k}, \mathbf{p}}\right|^{2} \int_{-\infty}^{\infty} \frac{d \omega}{2 \pi}, \\
A_{N}(\mathbf{k}, \omega) A_{S}(\mathbf{p}, \omega+\mathrm{eV})[f(\omega)-f(\omega+\mathrm{eV})] .
\end{gathered}
$$

Here, $A_{N, S}(\mathbf{k}, \omega)=-2 \operatorname{Im} G_{N, S}(\mathbf{k}, \omega)$ are the spectral functions of the normal metal and superconductor, respectively, and $f(\omega)$ is the Fermi distribution function. Since the angular and energy dependence of the tunnelling matrix elements $\left|T_{\mathbf{k}, \mathbf{p}}\right|^{2}$ is practically unimportant for $s$-wave superconductors, then the relative conductance $\sigma_{\mathrm{NS}}(V) \equiv$ $G_{\mathrm{NS}}(V) / G_{\mathrm{NN}}(V)$ is proportional to the tunnelling density of states $N_{T}(\omega)=\int A_{S}(\mathbf{k}, \omega) d^{3} k /(2 \pi)^{3}$, that is, $\sigma_{\mathrm{NS}}(\omega) \approx N_{T}(\omega)$ where

$$
N_{T}(\omega)=\operatorname{Re}\left\{\frac{\omega+i \tilde{\gamma}(\omega)}{\sqrt{(\omega+i \tilde{\gamma}(\omega))^{2}-\tilde{Z}^{2}(\omega) \Delta(\omega)^{2}}}\right\} .
$$

Here, $\tilde{Z}(\omega)=Z(\omega) / \operatorname{Re} Z(\omega), \tilde{\gamma}(\omega)=\gamma(\omega) / \operatorname{Re} Z(\omega), Z(\omega)=$ $\operatorname{Re} Z(\omega)+i \gamma(\omega) / \omega$, and the quasiparticle scattering rate in the superconducting state $\gamma_{s}(\omega, T)=-2 \operatorname{Im} \Sigma(\omega, T)$ is given by

$$
\begin{aligned}
\gamma_{s}(\omega, T)= & 2 \pi \int_{0}^{\infty} d \nu \alpha^{2} F(\nu) N_{s}(\nu+\omega) \\
& \times\left\{2 n_{B}(\nu)+n_{F}(\nu+\omega)+n_{F}(\nu-\omega)\right\}+\gamma^{\mathrm{imp}},
\end{aligned}
$$


where $N_{s}(\omega)=\operatorname{Re}\left\{\omega /\left(\omega^{2}-\Delta^{2}(\omega)\right)^{1 / 2}\right.$ is the quasiparticle density of states in the superconducting state; $n_{B, F}(v)$ are Bose and Fermi distribution function, respectively. Since the structure of the phonon spectrum is contained in $\alpha^{2} F(\omega)$, it is reflected on $\Delta(\omega)$ for $\omega>\Delta_{0}$ (the real gap obtained from $\left.\Delta_{0}=\operatorname{Re} \Delta\left(\omega=\Delta_{0}\right)\right)$ which gives the structure in $G_{S}(V)$ at $V=\Delta_{0}+\omega_{\mathrm{ph}}$. On the contrary one can extract the spectral function $\alpha^{2} F(\omega)$ from $G_{N S}(V)$ by the inversion procedure proposed by Kulić [6] and McMillan and Rowell [228]. It turns out that in low-temperature superconductors the peaks of $-d^{2} I / d V^{2}$ at $e V_{i}=\Delta+$ $\omega_{\mathrm{ph}, i}$ correspond to the peak positions of $\alpha^{2} F(\omega)$ and $F(\omega)$. However, we would like to point out that in HTSC cuprates the gap function is unconventional and very anisotropic, that is, $\Delta\left(\mathbf{k}, i \omega_{n}\right) \sim \cos k_{x} a-\cos k_{y} a$. Since in this case the extraction of $\alpha^{2} F\left(\mathbf{k}, \mathbf{k}^{\prime}, \omega\right)$ is difficult and at present rather unrealistic task, then an "average" $\alpha^{2} F(\omega)$ is extracted from the experimental curve $G_{S}(V)$. There is belief that it gives relevant information on the real spectral function such as the energy width of the bosonic spectrum $\left(0<\omega<\omega_{\max }\right)$ and positions and distributions of peaks due to bosons. It turns out that even such an approximate procedure gives valuable information in HTSC cuprates-see discussion in Section 1.3.4.

Note that in the case when both EPI and spin-fluctuation interaction (SFI) are present one should make difference between $\lambda_{\mathbf{k p}}^{Z}\left(i v_{n}\right)$ and $\lambda_{\mathbf{k p}}^{\Delta}\left(i v_{n}\right)$ defined by

$$
\begin{gathered}
\lambda_{\mathbf{k p}}^{Z}\left(i \nu_{n}\right)=\lambda_{\mathrm{sf}, \mathbf{k p}}\left(i \nu_{n}\right)+\lambda_{e p, \mathbf{k p}}\left(i \nu_{n}\right), \\
\lambda_{\mathbf{k p}}^{\Delta}=\lambda_{e p, \mathbf{k p}}\left(i \nu_{n}\right)-\lambda_{\mathrm{sf}, \mathbf{k p}}\left(i \nu_{n}\right) .
\end{gathered}
$$

In absence of EPI, $\lambda_{\mathbf{k p}}^{Z}\left(i v_{n}\right)$ and $\lambda_{\mathbf{k p}}^{\Delta}\left(i \nu_{n}\right)$ differ by sign, that is, $\lambda_{\mathbf{k p}}^{Z}\left(i \nu_{n}\right)=-\lambda_{\mathbf{k p}}^{\Delta}\left(i \nu_{n}\right)>0$ since the SFI potential is repulsive in the singlet pairing channel.

a. Inversion of Tunnelling Data. Phonon features in the conductance $\sigma_{\mathrm{NS}}(V)$ at $\mathrm{eV}=\Delta_{0}+\omega_{\mathrm{ph}}$ make the tunnelling spectroscopy a powerful method in obtaining the Eliashberg spectral function $\alpha^{2} F(\omega)$. Two methods were used in the past for extracting $\alpha^{2} F(\omega)$.

The first method is based on solving the inverse problem of the nonlinear Eliashberg equations. Namely, by measuring $\sigma_{\mathrm{NS}}(V)$, one obtains the tunnelling density of states $N_{T}(\omega)(\sim$ $\left.\sigma_{\mathrm{NS}}(\omega)\right)$ and by the inversion procedure one obtains $\alpha^{2} F(\omega)$ [228]. In reality the method is based on the iteration procedure - the McMillan-Rowell $(M R)$ inversion, where in the first step an initial $\alpha^{2} F_{\text {ini }}(\omega), \mu_{\text {ini }}^{*}$, and $\Delta_{\text {ini }}(\omega)$ are inserted into Eliashberg equations (e.g., $\Delta_{\text {ini }}(\omega)=\Delta_{0}$ for $\omega<\omega_{0}$ and $\Delta_{\text {ini }}(\omega)=0$ for $\left.\omega>\omega_{0}\right)$ and then $\sigma_{\text {ini }}(V)$ is calculated. In the second step the functional derivative $\delta \sigma(\omega) / \delta \alpha^{2} F(\omega)(\omega \equiv$ $\mathrm{eV})$ is found in the presence of a small change of $\alpha^{2} F_{\text {ini }}(\omega)$ and then the iterated solution $\alpha^{2} F_{(1)}(\omega)=\alpha^{2} F_{\text {ini }}(\omega)+$ $\delta \alpha^{2} F(\omega)$ is obtained, where the correction $\delta \alpha^{2} F(\omega)$ is given by

$$
\delta \alpha^{2} F(\omega)=\int d \nu\left[\frac{\delta \sigma_{\mathrm{ini}}(V)}{\delta \alpha^{2} F(\nu)}\right]^{-1}\left[\sigma_{\exp }(\nu)-\sigma_{\mathrm{ini}}(\nu)\right] .
$$

The procedure is iterated until $\alpha^{2} F_{(n)}(\omega)$ and $\mu_{(n)}^{*}$ converge to $\alpha^{2} F(\omega)$ and $\mu^{*}$ which reproduce the experimentally obtained conductance $\sigma_{\mathrm{NS}}^{\exp }(V)$. In such a way the obtained $\alpha^{2} F(\omega)$ for $P b$ resembles the phonon density of states $F_{P b}(\omega)$, which is obtained from neutron scattering measurements. Note that the method depends explicitly on $\mu^{*}$ but on the contrary it requires only data on $\sigma_{\mathrm{NS}}(V)$ up to the voltage $V_{\max }=\omega_{\mathrm{ph}}^{\max }+\Delta_{0}$ where $\omega_{\mathrm{ph}}^{\max }$ is the maximum phonon energy $\left(\alpha^{2} F(\omega)=0\right.$ for $\left.\omega>\omega_{\mathrm{ph}}^{\max }\right)$ and $\Delta_{0}$ is the zero-temperature superconducting gap. One pragmatical feature for the interpretation of tunnelling spectra (and for obtaining the spectral pairing function $\alpha^{2} F(\omega)$ ) in LTS and HTSC cuprates is that the negative peaks of $d^{2} I / d V^{2}$ (or peaks in $\left.-d^{2} I / d V^{2}\right)$ are at the peak positions of $\alpha^{2} F(\omega)$ and $F(\omega)$. This feature will be discussed later on in relation with experimental situation in cuprates.

The second method has been invented in $[229,230]$ and it is based on the combination of the Eliashberg equations and dispersion relations for Green's functions-we call it GDS method. First, the tunnelling density of states is extracted from the tunnelling conductance in a more rigorous way [231]:

$$
\begin{aligned}
N_{T}(V)= & \frac{\sigma_{\mathrm{NS}}(V)}{\sigma_{\mathrm{NN}}(V)}-\frac{1}{\sigma^{*}(V)} \int_{0}^{V} d u \\
& \times \frac{d \sigma^{*}(u)}{d u}\left[N_{T}(V-u)-N_{T}(V)\right],
\end{aligned}
$$

where $\sigma^{*}(V)=\exp \{-\beta V\} \sigma_{\mathrm{NN}}(V)$ and the constant $\beta$ are obtained from $\sigma_{\mathrm{NN}}(V)$ at large biases—see $[229,230] . N_{T}(V)$ under the integral can be replaced by the BCS density of states. Since the second method is used in extracting $\alpha^{2} F(\omega)$ in a number of LTSC as well as in HTSC cuprates-see below-we describe it briefly for the case of isotropic EPI at $T=0 \mathrm{~K}$. In that case the Eliashberg equations are given by $[70,225-227,229,230]$ :

$$
\begin{aligned}
Z(\omega) \Delta(\omega)= & \int_{\Delta_{0}}^{\infty} d \omega^{\prime} \operatorname{Re}\left[\frac{\Delta\left(\omega^{\prime}\right)}{\left[\omega^{\prime 2}-\Delta^{2}\left(\omega^{\prime}\right)\right]^{1 / 2}}\right] \\
& \times\left[K_{+}\left(\omega, \omega^{\prime}\right)-\mu^{*} \theta\left(\omega_{c}-\omega\right)\right], \\
1-Z(\omega)= & \frac{1}{\omega} \int_{\Delta_{0}}^{\infty} d \omega^{\prime} \operatorname{Re}\left[\frac{\omega^{\prime}}{\left[\omega^{\prime 2}-\Delta^{2}\left(\omega^{\prime}\right)\right]^{1 / 2}}\right] K_{-}\left(\omega, \omega^{\prime}\right),
\end{aligned}
$$

where

$$
\begin{aligned}
K_{ \pm}\left(\omega, \omega^{\prime}\right)= & \int_{\Delta_{0}}^{\omega_{\mathrm{ph}}^{\max }} d \nu \alpha^{2} F(\nu) \\
& \times\left(\frac{1}{\omega^{\prime}+\omega+v+i 0^{+}} \pm \frac{1}{\omega^{\prime}-\omega+\nu-i 0^{+}}\right) .
\end{aligned}
$$

Here $\mu^{*}$ is the Coulomb pseudopotential, the cutoff $\omega_{c}$ is approximately $(5-10) \omega_{\mathrm{ph}}^{\max }$, and $\Delta_{0}=\Delta\left(\Delta_{0}\right)$ is the energy gap. Now by using the dispersion relation for the matrix Green's functions $\widehat{G}\left(\mathbf{k}, \omega_{n}\right)$ one obtains $[229,230]$

$$
\operatorname{Im} S(\omega)=\frac{2 \omega}{\pi} \int_{\Delta_{0}}^{\infty} d \omega^{\prime} \frac{N_{T}\left(\omega^{\prime}\right)-N_{\mathrm{BCS}}\left(\omega^{\prime}\right)}{\omega^{2}-\omega^{\prime 2}},
$$


where $S(\omega)=\omega /\left[\omega^{2}-\Delta^{2}(\omega)\right]^{1 / 2}$. From (A.13) one obtains

$$
\begin{array}{rl}
\int_{0}^{\omega-\Delta_{0}} & d \nu \alpha^{2} F(\omega-\nu) \operatorname{Re}\left\{\Delta(\nu)\left[\nu^{2}-\Delta^{2}(\nu)\right]^{1 / 2}\right\} \\
= & \frac{\operatorname{Re} \Delta(\omega)}{\omega} \int_{0}^{\omega-\Delta_{0}} d \nu \alpha^{2} F(\nu) N_{T}(\omega-\nu)+\frac{\operatorname{Im} \Delta(\omega)}{\pi} \\
& +\frac{\operatorname{Im} \Delta(\omega)}{\pi} \int_{0}^{\infty} d \omega^{\prime} N_{T}\left(\omega^{\prime}\right) \int_{0}^{\omega_{\mathrm{ph}}^{\max }} d \nu \frac{2 \alpha^{2} F(\nu)}{\left(\omega^{\prime}+\nu\right)^{2}-\omega^{2}}
\end{array}
$$

Based on (A.12)-(A.16) one obtains the scheme for extracting $\alpha^{2} F(\omega)$ :

$$
\begin{gathered}
\sigma_{\mathrm{NS}}(V), \sigma_{\mathrm{NN}}(V) \longrightarrow N_{T}(V), \\
\longrightarrow \operatorname{Im} S(\omega) \longrightarrow \Delta(\omega) \longrightarrow \alpha^{2} F(\omega) .
\end{gathered}
$$

The advantage in this method is that the explicit knowledge of $\mu^{*}$ is not required $[229,230]$. However, the integral equation for $\alpha^{2} F(\omega)$ is linear Fredholm equation of the first kind which is ill defined-see the discussion in Section 1.3.2 item (2)

b. Phonon Effects in $N_{T}(\omega)$. We briefly discuss the physical origin for the phonon effects in $N_{T}(\omega)$ by considering a model with only one peak, at $\omega_{0}$, in the phonon density of states $F(\omega)$ by assuming for simplicity $\mu^{*}=0$ and neglecting the weak structure in $N_{T}(\omega)$ at $n \omega_{0}+\Delta_{0}$, which is due to the nonlinear structure of the Eliashberg equations [232]. In Figure 37 it is seen that the real part of the gap function $\Delta_{R}(\omega)$ reaches a maximum at $\omega_{0}+\Delta_{0}$ then decreases and becomes negative and zero, while $\Delta_{I}(\omega)$ is peaked slightly beyond $\omega_{0}+\Delta_{0}$ that is the consequence of the effective electron-electron interaction via phonons.

It follows that for $\omega<\omega_{0}+\Delta_{0}$ most phonons have higher energies than the energy $\omega$ of electronic charge fluctuations and there is overscreening of this charge by the ions giving rise to attraction. For $\omega \approx \omega_{0}+\Delta_{0}$ the charge fluctuations are in resonance with ion vibrations giving rise to the peak in $\Delta_{R}(\omega)$. For $\omega_{0}+\Delta_{0}<\omega$ the ions move out of phase with respect to the charge fluctuations giving rise to repulsion and negative $\Delta_{R}(\omega)$. This is shown in Figure 37(b). The structure in $\Delta(\omega)$ is reflected on $N_{T}(\omega)$ as shown in Figure $37(\mathrm{c})$ which can be reconstructed from the approximate formula for $N_{T}(\omega)$ expanded in powers of $\Delta / \omega$ :

$$
\frac{N_{T}(\omega)}{N(0)} \approx 1+\frac{1}{2}\left[\left(\frac{\Delta_{R}(\omega)}{\omega}\right)^{2}-\left(\frac{\Delta_{I}(\omega)}{\omega}\right)^{2}\right]
$$

As $\Delta_{R}(\omega)$ increases above $\Delta_{0}$, this gives $N_{T}(\omega)>N_{\text {BCS }}(\omega)$, while for $\omega \gtrsim \omega_{0}+\Delta_{0}$ the real value $\Delta_{R}(\omega)$ decreases while $\Delta_{I}(\omega)$ rises and $N_{T}(\omega)$ decreases giving rise for $N_{T}(\omega)<$ $N_{\text {BCS }}(\omega)$.

A.2. Transport Spectral Function $\alpha_{t r}^{2} F(\omega)$. The spectral function $\alpha_{\mathrm{tr}}^{2} F(\omega)$ enters the dynamical conductivity $\sigma_{i j}(\omega)(i, j=$

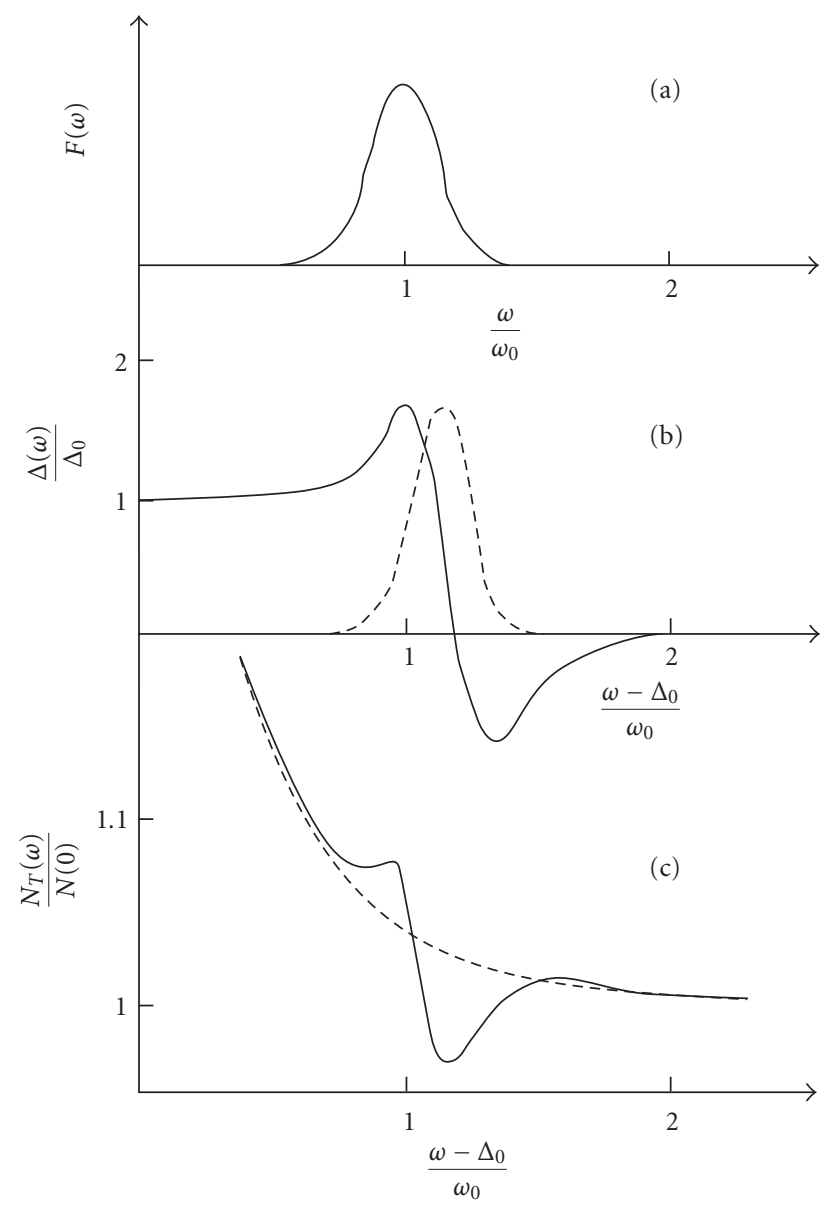

FIgURE 37: (a) Model phonon density of states $F(\omega)$ with the peak at $\omega_{0}$. (b) The real (solid) $\Delta_{R}$ and imaginary (dashed) part $\Delta_{I}$ of the gap $\Delta(\omega)$. (c) The normalized tunnelling density of states $N_{T}(\omega) / N(0)$ (solid) compared with the BCS density of states (dashed). From [232].

$a, b, c$ axis in HTS systems) which generally speaking is a tensor quantity given by the formula

$$
\begin{aligned}
\sigma_{i j}(\omega)=-\frac{e^{2}}{\omega} \int & \frac{d^{4} q}{(2 \pi)^{4}} \gamma_{i}(q, k+q) \\
& \times G(k+q) \Gamma_{j}(q, k+q) G(q),
\end{aligned}
$$

where $q=(\mathbf{q}, v)$ and $k=(\mathbf{k}=0, \omega)$ and the bare current vertex $\gamma_{i}(q, k+q ; \mathbf{k}=0)$ is related to the Fermi velocity $v_{F, i}$, that is, $\gamma_{i}(q, k+q ; \mathbf{k}=0)=v_{F, i}$. The vertex function $\Gamma_{j}(q, k+q)$ takes into account the renormalization due to all scattering processes responsible for finite conductivity [233]. In the following we study only the in-plane conductivity at $\mathbf{k}=0$. The latter case is realized due to the fact that the long penetration depth in HTSC cuprates and the skin depth in the normal state are very large. In the EPI theory, $\Gamma_{j}(q, k+$ $q) \equiv \Gamma_{j}\left(\mathbf{q}, i \omega_{n}, i \omega_{n}+i \omega_{m}\right)$ is the solution of an approximative integral equation written in the symbolic form [118] $\Gamma_{j}=$ $v_{j}+V_{\text {eff }} G G \Gamma_{j}$. The effective potential $V_{\text {eff }}$ (due to EPI) is given by $V_{\text {eff }}=\sum_{\kappa}\left|g_{\kappa}^{\text {ren }}\right|{ }^{2} D_{\kappa}$, where $D_{\kappa}$ is the phonon Green's 
function. In such a case the Kubo theory predicts $\sigma_{i i}^{\text {intra }}(\omega)$ $(i=x, y, z)$ :

$$
\begin{aligned}
\sigma_{i i}(\omega)=\frac{\omega_{p, i i}^{2}}{4 i \pi \omega}\left\{\int_{-\omega}^{0} d \nu \operatorname{th}\left(\frac{\omega+\nu}{2 T}\right) S^{-1}(\omega, \nu)\right. \\
\left.\quad+\int_{0}^{\infty} d \nu\left[\operatorname{th}\left(\frac{\omega+\nu}{2 T}\right)-\operatorname{th}\left(\frac{\nu}{2 T}\right)\right] S^{-1}(\omega, \nu)\right\},
\end{aligned}
$$

where $S(\omega, \nu)=\omega+\Sigma_{\mathrm{tr}}^{*}(\omega+\nu)-\Sigma_{\mathrm{tr}}(\nu)+i \gamma_{\mathrm{tr}}^{\mathrm{imp}}$, and $\gamma_{\mathrm{tr}}^{\mathrm{imp}}$ is the impurity contribution. In the following we omit the tensor index $i i$ in $\sigma_{i i}(\omega)$. In the presence of several bosonic scattering processes the transport self-energy $\Sigma_{\mathrm{tr}}(\omega)=\operatorname{Re} \Sigma_{\mathrm{tr}}(\omega)+$ $i \operatorname{Im} \Sigma_{\mathrm{tr}}(\omega)$ is given by

$$
\begin{gathered}
\Sigma_{\operatorname{tr}}(\omega)=-\sum_{l} \int_{0}^{\infty} d \nu \alpha_{\mathrm{tr}, l}^{2} F_{l}(\nu)\left[K_{1}(\omega, \nu)+i K_{2}(\omega, \nu)\right], \\
K_{1}(\omega, \nu)=\operatorname{Re}\left[\Psi\left(\frac{1}{2}+i \frac{\omega+v}{2 \pi T}\right)-\Psi\left(\frac{1}{2}+i \frac{\omega-v}{2 \pi T}\right)\right], \\
K_{2}(\omega, \nu)=\frac{\pi}{2}\left[2 c \operatorname{th}\left(\frac{v}{2 T}\right)-\operatorname{th}\left(\frac{\omega+\nu}{2 T}\right)+\operatorname{th}\left(\frac{\omega-v}{2 T}\right)\right] .
\end{gathered}
$$

Here $\alpha_{\mathrm{tr}, l}^{2} F_{l}(\nu)$ is the transport spectral function which measures the strength of the $l$ th (bosonic) scattering process and $\Psi$ is the di-gamma function. The index $l$ enumerates EPI, charge, and spin-fluctuation scattering processes. Like in the case of EPI, the transport bosonic spectral function $\alpha_{\mathrm{tr}, l}^{2} F(\Omega)$ defined in (97) is given explicitly by

$$
\begin{aligned}
\alpha_{\mathrm{tr}, l}^{2} F(\omega)= & \frac{1}{N^{2}(\mu)} \int \frac{d S_{\mathbf{k}}}{v_{F, \mathbf{k}}} \int \frac{d S_{\mathbf{k}^{\prime}}}{v_{F, \mathbf{k}^{\prime}}} \\
& \times\left[1-\frac{v_{F, \mathbf{k}}^{i} v_{F, \mathbf{k}}^{i}}{\left(v_{F, \mathbf{k}}^{i}\right)^{2}}\right] \alpha_{\mathbf{k k}^{\prime}, l}^{2} F(\omega) .
\end{aligned}
$$

We stress that in the phenomenological SFI theory [12-17] one assumes that $\alpha_{\mathbf{k k}^{\prime}}^{2} F(\omega) \approx N(\mu) g_{\text {sf }}^{2} \operatorname{Im} \chi(\mathbf{k}-\mathbf{p}, \omega)$, which, as we have repeated several times, can be justified only for small $g_{\text {sf }}$, that is, $g_{\text {sf }} \ll W_{b}$ (the bandwidth).

In case of weak coupling $(\lambda<1), \sigma(\omega)$ can be written in the generalized (extended) Drude form as discussed in Section 1.3.2.

\section{Acknowledgments}

The authors devote this paper to their great teacher and friend Vitalii Lazarevich Ginzburg who passed away recently. His permanent interest in their work and support in many respects over many years are unforgettable. M. L. Kulić is thankful to Karl Bennemann for inspiring discussions on many subjects related to physics of HTSC cuprates. They also thank Godfrey Akpojotor for careful reading of the manuscript. M. L. Kulić is thankful to the Max-Born-Institut für Nichtlineare Optik und Kurzzeitspektroskopie, Berlin, for the hospitality and financial support during his stay where part of this work has been done.

\section{References}

[1] A. B. Migdal, "Interaction between electrons and the lattice vibrations in a normal metal," Soviet Physics: Journal of Experimental and Theoretical Physics, vol. 34, p. 996, 1958.

[2] O. V. Danylenko and O. V. Dolgov, "Nonadiabatic contribution to the quasiparticle self-energy in systems with strong electron-phonon interaction," Physical Review B, vol. 63, Article ID 094506, 9 pages, 2001.

[3] V. L. Ginzburg and E. G. Maksimov, "Mechanisms and models of high temperature superconductors," Physica C, vol. 235-240, pp. 193-196, 1994.

[4] V. L. Ginzburg and E. G. Maksimov, "Superconductivity," Fizika Khimiya Tekhnika, vol. 5, p. 1505, 1992 (Russian).

[5] E. G. Maksimov, "High-temperature superconductivity: the current state," Uspekhi Fizicheskikh Nauk, vol. 170, p. 1033, 2000.

[6] M. L. Kulić, "Interplay of electron-phonon interaction and strong correlations: the possible way to high-temperature superconductivity," Physics Report, vol. 338, no. 1-2, pp. 1264, 2000.

[7] C. Falter, "Phonons, electronic charge response and electronphonon interaction in the high-temperature superconductors," Physica Status Solidi (B), vol. 242, p. 118, 2005.

[8] A. S. Alexandrov, "Unconventional pairs glued by conventional phonons in cuprate superconductors," Journal of Superconductivity and Novel Magnetism, vol. 22, no. 2, pp. 103-107, 2009.

[9] T. M. Hardy, J. P. Hague, J. H. Samson, and A. S. Alexandrov, "Superconductivity in a Hubbard-Froehlich model and in cuprates," Physical Review B, vol. 79, Article ID 212501, 2009.

[10] O. Gunnarsson and O. Rösch, "Interplay between electronphonon and Coulomb interactions in cuprates," Journal of Physics, vol. 20, no. 4, Article ID 043201, 2008.

[11] O. Rösch, J. E. Hahn, O. Gunnarsson, and V. H. Crespi, "Phonons, electronic charge response and electron-phonon interaction in the high-temperature superconductors," Physica Status Solidi (B), vol. 242, p. 78, 2005.

[12] A. J. Millis, H. Monien, and D. Pines, "Phenomenological model of nuclear relaxation in the normal state of $\mathrm{YBa}_{2} \mathrm{Cu}_{3} \mathrm{O}_{7}$," Physical Review B, vol. 42, no. 1, pp. 167-178, 1990.

[13] P. Monthoux and D. Pines, "Spin-fluctuation-induced superconductivity in the copper oxides: a strong coupling calculation," Physical Review Letters, vol. 69, no. 6, pp. 961-964, 1992.

[14] P. Monthoux and D. Pines, " $\mathrm{YBa}_{2} \mathrm{Cu}_{3} \mathrm{O}_{7}$ : a nearly antiferromagnetic Fermi liquid," Physical Review B, vol. 47, no. 10, pp. 6069-6081, 1993.

[15] B. P. Stojković and D. Pines, "Theory of the optical conductivity in the cuprate superconductors," Physical Review B, vol. 56, no. 18, pp. 11931-11941, 1997.

[16] D. Pines, preprint CNSL Newsletter, LALP-97-010, no. 138, June 1997.

[17] D. Pines, "Spin excitations and superconductivity in cuprate oxide and heavy electron superconductors," Physica B, vol. 163, no. 1-3, pp. 78-88, 1990.

[18] P. A. Lee, N. Nagaosa, and C.-G. Wen, "Doping a Mott insulator: physics of high-temperature superconductivity," Reviews of Modern Physics, vol. 78, p. 17, 2006.

[19] T. Aimi and M. Imada, "Does simple two-dimensional Hubbard model account for high- $T_{c}$ superconductivity in copper oxides?" Journal of the Physical Society of Japan, vol. 76, no. 11, Article ID 113708, 4 pages, 2007. 
[20] D. J. Scalapino, S. R. White, and S. C. Zhang, "Superfluid density and the drude weight of the hubbard model," Physical Review Letters, vol. 68, no. 18, pp. 2830-2833, 1992.

[21] L. P. Pryadko, S. A. Kivelson, and O. Zachar, "Incipient order in the t-J model at high temperatures," Physical Review Letters, vol. 92, no. 6, Article ID 067002, 4 pages, 2004.

[22] A. Damascelli, Z. Hussain, and Z.-X. Shen, "Angle-resolved photoemission studies of the cuprate superconductors," Reviews of Modern Physics, vol. 75, no. 2, pp. 473-541, 2003.

[23] J. C. Campuzano, M. R. Norman, and M. Randeria, "Photoemission in the high $T_{c}$ superconductors," in Physics of Conventional and Unconventional Superconductors Vol. II, K. H. Bennemann and J. B. Ketterson, Eds., pp. 167-273, Springer, Berlin, Germany, 2004.

[24] E. Schachinger and J. P. Carbotte, "Comparison of the inand out-of-plane charge dynamics in $\mathrm{YBa}_{2} \mathrm{Cu}_{3} \mathrm{O}_{6.95}$," Physical Review B, vol. 64, no. 9, Article ID 094501, 10 pages, 2001.

[25] J. Hwang, E. Schachinger, J. P. Carbotte, F. Gao, D. B. Tanner, and T. Timusk, "Bosonic spectral density of epitaxial thin-film $\mathrm{La}_{1.83} \mathrm{Sr}_{0.17} \mathrm{CuO}_{4}$ superconductors from infrared conductivity measurements," Physical Review Letters, vol. 100, no. 13, Article ID 137005, 2008.

[26] J. Hwang, T. Timusk, E. Schachinger, and J. P. Carbotte, "Evolution of the bosonic spectral density of the hightemperature superconductor $\mathrm{Bi}_{2} \mathrm{Sr}_{2} \mathrm{CaCu}_{2} \mathrm{O}_{8+\delta}$," Physical Review B, vol. 75, no. 14, Article ID 144508, 2007.

[27] J. Hwang, T. Timusk, and G. D. Gu, "High-transitiontemperature superconductivity in the absence of the magnetic-resonance mode," Nature, vol. 427, no. 6976, pp. 714-717, 2004.

[28] R. Heid, K.-P. Bohnen, R. Zeyher, and D. Manske, "Momentum dependence of the electron-phonon coupling and selfenergy effects in superconducting $\mathrm{YBa}_{2} \mathrm{Cu}_{3} \mathrm{O}_{7}$ within the local density approximation," Physical Review Letters, vol. 100, no. 13, Article ID 137001, 2008.

[29] F. Giustino, M. L. Cohen, and S. G. Louie, "Small phonon contribution to the photoemission kink in the copper oxide superconductors," Nature, vol. 452, no. 7190, pp. 975-978, 2008.

[30] Ph. Bourges, "From magnons to the resonance peak: spin dynamics in high- $T_{c}$ superconducting cuprates by inelastic neutron scattering," http://arxiv.org/abs/cond-mat/9901333.

[31] D. Reznik, J.-P. Ismer, I. Eremin, et al., "Local-moment fluctuations in the optimally doped high- $T_{c}$ superconductor $\mathrm{YBa}_{2} \mathrm{Cu}_{3} \mathrm{O}_{6.95}$," Physical Review $B$, vol. 78, no. 13, Article ID 132503, 2008.

[32] P. B. Allen, "Electron-phonon effects in the infrared properties of metals," Physical Review B, vol. 3, no. 2, pp. 305-320, 1971.

[33] O. V. Dolgov and S. V. Shulga, "Analysis of intermediate boson spectra from FIR data for HTSC and heavy fermion systems," Journal of Superconductivity, vol. 8, no. 5, pp. 611612, 1995.

[34] S. V. Shulga, O. V. Dolgov, and E. G. Maksimov, "Electronic states and optical spectra of HTSC with electron-phonon coupling," Physica C, vol. 178, no. 4-6, pp. 266-274, 1991.

[35] O. V. Dolgov, E. G. Maksimov, and S. V. Shulga, in ElectronPhonon Interaction in Oxide Superconductors, R. Baquero, Ed., p. 30, World Scientific, Singapore, 1991.

[36] S. V. Shulga, "Electron-boson effects in the infrared properties of metals," in High- $T_{c}$ Superconductors and Related Materials, S.-L. Drechsler and T. Mishonov, Eds., p. 323, Kluwer Academic Publishers, 2001.
[37] M. L. Kulić, Lectures on the Physics of Highly Correlated Electron Systems, vol. 715 of AIP Conference Proceedings, 2004.

[38] M. L. Kulić and O. V. Dolgov, "Forward scattering peak in the electron-phonon interaction and impurity scattering of cuprate superconductors," Physica Status Solidi (B), vol. 242, no. 1, pp. 151-178, 2005.

[39] A. E. Karakozov, E. G. Maksimov, and O. V. Dolgov, "Electromagnetic response of superconductors and optical sum rule," Solid State Communications, vol. 124, no. 4, pp. 119-124, 2002.

[40] A. E. Karakozov and E. G. Maksimov, "Optical sum rule in metals with a strong interaction," Solid State Communications, vol. 139, no. 2, pp. 80-85, 2006.

[41] P. Kusar, V. V. Kabanov, S. Sugai, J. Demsar, T. Mertelj, and D. Mihailovic, "Controlled vaporization of the superconducting condensate in cuprate superconductors sheds light on the pairing boson," Physical Review Letters, vol. 101, Article ID 227001, 2008.

[42] L. N. Bulaevskii, O. V. Dolgov, I. P. Kazakov, et al., "A tunnelling study of the oxide superconductors $\mathrm{La}_{2-x} \mathrm{Sr}_{x} \mathrm{CuO}_{4-y}$ and $\mathrm{EuBa}_{2} \mathrm{Cu}_{3} \mathrm{O}_{7}$," Superconductor Science and Technology, vol. 1, no. 4, pp. 205-209, 1988.

[43] S. I. Vedeneev, A. G. M. Jansen, P. Samuely, V. A. Stepanov, A. A. Tsvetkov, and P. Wyder, "Tunneling in the ab plane of the high- $T_{c}$ superconductor $\mathrm{Bi}_{2} \mathrm{Sr}_{2} \mathrm{CaCu}_{2} \mathrm{O}_{8+\delta}$ in high magnetic fields," Physical Review B, vol. 49, no. 14, pp. 9823-9830, 1994.

[44] S. I. Vedeneev, A. G. M. Jansen, A. A. Tsvetkov, and P. Wyder, "Bloch-Grüneisen behavior for the in-plane resistivity of $\mathrm{Bi}_{2} \mathrm{Sr}_{2} \mathrm{CuO}_{x}$ single crystals," Physical Review B, vol. 51, no. 22, pp. 16380-16383, 1995.

[45] S. I. Vedeneev, A. G. M. Jansen, and P. Wyder, "Tunneling spectroscopy of $\mathrm{Bi}_{2} \mathrm{Sr}_{2} \mathrm{CaCu}_{2} \mathrm{O}_{8}$ single crystals," Physica B, vol. 218, no. 1-4, pp. 213-216, 1996.

[46] D. Shimada, Y. Shiina, A. Mottate, Y. Ohyagi, and N. Tsuda, "Phonon structure in the tunneling conductance of $\mathrm{Bi}_{2} \mathrm{Sr}_{2} \mathrm{CaCu}_{2} \mathrm{O}_{8}$," Physical Review B, vol. 51, no. 22, pp. 16495-16498, 1995.

[47] N. Miyakawa, A. Nakamura, Y. Fujino, et al., "Electronphonon spectral function $\alpha^{2} F(\omega)$ ) determined by quasiparticle tunneling spectroscopy for $\mathrm{Bi}_{2} \mathrm{Sr}_{2} \mathrm{CaCu}_{2} \mathrm{O}_{8} / \mathrm{Au}$ junctions," Physica C, vol. 282-287, pp. 1519-1520, 1997.

[48] N. Miyakawa, Y. Shiina, T. Kaneko, and N. Tsuda, "Analysis of phonon structures in the tunneling conductance of Bicuprates," Journal of the Physical Society of Japan, vol. 62, pp. 2445-2455, 1993.

[49] N. Miyakawa, Y. Shiina, T. Kido, and N. Tsuda, "Fine structure in the tunneling conductance of a $\mathrm{Bi}_{2} \mathrm{Sr}_{2} \mathrm{CaCu}_{2} \mathrm{O}_{8}$ GaAs junction," Journal of the Physical Society of Japan, vol. 58, pp. 383-386, 1989.

[50] Y. Shiina, D. Shimada, A. Mottate, Y. Ohyagi, and N. Tsuda, "Temperature dependence of the tunneling conductance of $\mathrm{Bi}_{2} \mathrm{Sr}_{2} \mathrm{CaCu}_{2} \mathrm{O}_{8}$ : phonon contribution to high- $T_{c}$ superconductivity," Journal of the Physical Society of Japan, vol. 64, pp. 2577-2584, 1995.

[51] Y. Ohyagi, D. Shimada, N. Miyakawa, et al., "Reproducibility of phonon structures in the tunneling conductance of $\mathrm{Bi}_{2} \mathrm{Sr}_{2} \mathrm{CaCu}_{2} \mathrm{O}_{8}$," Journal of the Physical Society of Japan, vol. 64, pp. 3376-3383, 1995.

[52] R. S. Gonnelli, F. Asdente, and D. Andreone, "Reproducible inelastic tunneling in $\mathrm{Nb} / \mathrm{Bi}_{2} \mathrm{Sr}_{2} \mathrm{CaCu}_{2} \mathrm{O}_{8+x}$ point-contact junctions," Physical Review B, vol. 49, no. 2, pp. 1480-1483, 1994. 
[53] D. Shimada, N. Tsuda, U. Paltzer, and F. W. de Wette, "Tunneling phonon structures and the calculated phonon density of states for $\mathrm{Bi}_{2} \mathrm{Sr}_{2} \mathrm{CaCu}_{2} \mathrm{O}_{8}$," Physica C, vol. 298, no. 3-4, pp. 195-202, 1998.

[54] N. Tsuda, et al., "Contribution of the electron-phonon interaction to high- $T_{c}$ superconductivity: tunneling study of $\mathrm{Bi}_{2} \mathrm{Sr}_{2} \mathrm{CaCu}_{2} \mathrm{O}_{8}$," in New Research on Superconductivity, B. P. Martinis, Ed., pp. 105-141, Nova Science Publishers, 2007.

[55] Y. G. Ponomarev, et al., "Extended van Hove singularity, strong electron-phonon interaction and superconducting gap in doped Bi-2212 single crystal," Physica Status Solidi (B), vol. 66, p. 2072, 2009.

[56] H. Shim, P. Chaudhari, G. Logvenov, and I. Božović, "Electron-phonon interactions in superconducting $\mathrm{La}_{1.84} \mathrm{Sr}_{0.16} \mathrm{CuO}_{4}$ films," Physical Review Letters, vol. 101, no. 24, Article ID 247004, 2008.

[57] S. Sugai, S. Shamoto, M. Sato, T. Ido, H. Takagi, and S. Uchida, "Symmetry breaking on the phonon Raman spectra only at the superconductor compositions in $\mathrm{La}_{2-x} \mathrm{Sr}_{x} \mathrm{CuO}_{4}$," Solid State Communications, vol. 76, no. 3, pp. 371-376, 1990.

[58] F. Marsiglio, et al., "Eliashberg treatment of the microwave conductivity of niobium," Physical Review B, vol. 50, p. 7023, 1994.

[59] O. Klein, E. J. Nicol, K. Holczer, and G. Grüner, "Conductivity coherence factors in the conventional superconductors $\mathrm{Nb}$ and Pb," Physical Review B, vol. 50, no. 9, pp. 6307-6316, 1994.

[60] D. G. Hinks and J. D. Jorgensen, "The isotope effect and phonons in MgB2," Physica C, vol. 385, no. 1-2, pp. 98-104, 2003.

[61] K. McElroy, R. W. Simmonds, J. E. Hoffman, et al., "Relating atomic-scale electronic phenomena to wave-like quasiparticle states in superconducting $\mathrm{Bi}_{2} \mathrm{Sr}_{2} \mathrm{CaCu}_{2} \mathrm{O}_{8+\delta}$, Nature, vol. 422, no. 6932, pp. 592-596, 2003.

[62] J. Lee, J. L. V. Lewandowski, and T. G. Jenkins, "Transport, noise, and conservation properties in gyrokinetic plasmas," Bulletin of the American Physical Society, vol. 50, p. 299, 2005.

[63] J. C. Davis, et al., Bulletin of the American Physical Society, vol. 50, p. 1223, 2005.

[64] J. C. Phillips, "Superconductive excitations and the infrared vibronic spectra of BSCCO," Physica Status Solidi (B), vol. 242, no. 1, pp. 51-57, 2005.

[65] M. L. Cohen and P. W. Anderson, "Comments on the maximum superconducting transition temperature," in Superconductivity in $d$ and $f$ Band Metals, D. H. Douglass, Ed., AIP Conference Proceedings, p. 17, AIP, New York, NY, USA, 1972.

[66] P. W. Anderson, A Career in Theoretical Physics, World Scientific, Singapore, 1994.

[67] O. V. Dolgov and E. G. Maksimov, "A note on the possible mechanisms of high-temperature superconductivity," Uspekhi Fizicheskikh Nauk, vol. 177, p. 983, 2007, translated in Physics-Uspekhi, vol. 50, p. 933, 2007.

[68] D. A. Kirzhnitz, "General properties of electromagnetic response functions," in Dielectric Function of Condensed Systems, L. V. Keldysh, D. A. Kirzhnits, and A. A. Maradudin, Eds., chapter 2, Elsevier, Amsterdam, The Netherlands, 1989.

[69] V. L. Ginzburg and D. Kirzhnitz, Eds., High Temperature Superconductivity, Consultant Bureau, New York, NY, USA, 1982.

[70] P. B. Allen and B. Mitrović, Solid State Physics, vol. 37 of $H$. Ehrenreich, F. Seitz and D. Turnbull Ed., Academic, New York, NY, USA, 1982.
[71] O. V. Dolgov, D. A. Kirzhnits, and E. G. Maksimov, "On an admissible sign of the static dielectric function of matter," Reviews of Modern Physics, vol. 53, no. 1, pp. 81-93, 1981.

[72] O. V. Dolgov, D. A. Kirzhnits, and E. G. Maksimov, "Dielectric function and superconductivity," in Superconductivity, Superdiamagnetism and Superfluidity, V. L. Ginzburg, Ed., chapter 2, MIR, Moscow, Russia, 1987.

[73] O. V. Dolgov and E. G. Maksimov, "The dielectric function of crystalline systems," in Dielectric Function of Condensed Systems, L. V. Keldysh, D. A. Kirzhnits, and A. A. Maradudin, Eds., chapter 4, Elsevier, Amsterdam, The Netherlands, 1989.

[74] E. G. Maksimov and D. Yu. Savrasov, "Lattice stability and superconductivity of the metallic hydrogen at high pressure," Solid State Communications, vol. 119, no. 10-11, pp. 569-572, 2001.

[75] C. C. Tsuei and J. R. Kirtley, "Pairing symmetry in cuprate superconductors," Reviews of Modern Physics, vol. 72, no. 4, pp. 969-1016, 2000.

[76] A. I. Lichtenstein and M. L. Kulić, "Electron-boson interaction can help d wave pairing self-consistent approach," Physica C, vol. 245, no. 1-2, pp. 186-192, 1995.

[77] D. J. Scalapino, "The case for $\mathrm{dx} 2-\mathrm{y} 2$ pairing in the cuprate superconductors," Physics Report, vol. 250, no. 6, pp. 329365, 1995.

[78] M. L. Kulić and R. Zeyher, "Influence of strong electron correlations on the electron-phonon coupling in high- $T_{c}$ oxides," Physical Review B, vol. 49, no. 6, pp. 4395-4398, 1994.

[79] R. Zeyher and M. L. Kulić, "Electron-phonon coupling in the presence of strong correlations," Physica B, vol. 199-200, pp. 358-360, 1994.

[80] R. Zeyher and M. L. Kulić, "Modifications of the transport and the pairing electron-phonon coupling in high- $T_{c}$ oxides due to strong electronic correlations," Physica C, vol. 235240, pp. 2151-2152, 1994.

[81] T. Dahm, V. Hinkov, S. V. Borisenko, et al., "Strength of the spin-fluctuation-mediated pairing interaction in a hightemperature superconductor," Nature Physics, vol. 5, no. 3, pp. 217-221, 2009.

[82] H.-B. Schüttler and M. R. Norman, "Contrasting dynamic spin susceptibility models and their relation to hightemperature superconductivity," Physical Review B, vol. 54, no. 18, pp. 13295-13305, 1996.

[83] S. M. Hayden, H. A. Mook, P. Dal, T. G. Perring, and F. Doan, "The structure of the high-energy spin excitations in a high-transition-temperature superconductor," Nature, vol. 429, no. 6991, pp. 531-534, 2004.

[84] V. Hinkov, P. Bourges, S. Pailhès, et al., "Spin dynamics in the pseudogap state of a high-temperature superconductor," Nature Physics, vol. 3, no. 11, pp. 780-785, 2007.

[85] S. V. Borisenko, A. A. Kordyuk, V. Zabolotnyy, et al., "Kinks, nodal bilayer splitting, and interband scattering in $\mathrm{YBa}_{2} \mathrm{Cu}_{3} \mathrm{O}_{6+x}$," Physical Review Letters, vol. 96, no. 11, Article ID 117004, 4 pages, 2006.

[86] V. B. Zabolotnyy, S. V. Borisenko, A. A. Kordyuk, et al., "Momentum and temperature dependence of renormalization effects in the high-temperature superconductor $\mathrm{YBa}_{2} \mathrm{Cu}_{3} \mathrm{O}_{7-\delta}$," Physical Review B, vol. 76, no. 6, Article ID 064519, 2007.

[87] T. A. Maier, A. Macridin, M. Jarrell, and D. J. Scalapino, "Systematic analysis of a spin-susceptibility representation of the pairing interaction in the two-dimensional Hubbard model," Physical Review B, vol. 76, no. 14, Article ID 144516, 2007. 
[88] P. W. Anderson, "Is there glue in cuprate superconductors?" Science, vol. 317, p. 1705, 2007.

[89] H.-Y. Kee, S. A. Kivelson, and G. Aeppli, "Spin-1 neutron resonance peak cannot account for electronic anomalies in the cuprate superconductors," Physical Review Letters, vol. 88, no. 25, Article ID 257002, 4 pages, 2002.

[90] M. L. Kulić and I. M. Kulić, "High- $T_{c}$ superconductors with antiferromagnetic order: limitations on spin-fluctuation pairing mechanism," Physica C, vol. 391, no. 1, pp. 42-48, 2003.

[91] A. Lanzara, P. V. Bogdanov, X. J. Zhou, et al., "Evidence for ubiquitous strong electron-phonon coupling in hightemperature superconductors," Nature, vol. 412, no. 6846, pp. 510-514, 2001.

[92] T. Valla, A. V. Fedorov, P. D. Johnson, et al., "Evidence for quantum critical behavior in the optimally doped cuprate $\mathrm{Bi}_{2} \mathrm{Sr}_{2} \mathrm{CaCu}_{2} \mathrm{O}_{8+\delta}$," Science, vol. 285, no. 5436, pp. 2110 2113, 1999.

[93] G. Xu, G. D. Gu, M. Hücker, et al., "Testing the itinerancy of spin dynamics in superconducting $\mathrm{Bi}_{2} \mathrm{Sr}_{2} \mathrm{CaCu}_{2} \mathrm{O}_{8+\delta}$," Nature Physics, vol. 5, no. 9, pp. 642-646, 2009.

[94] I. Božović, "Plasmons in cuprate superconductors," Physical Review B, vol. 42, no. 4, pp. 1969-1984, 1990.

[95] Z. Schlesinger, R. T. Collins, F. Holtzberg, et al., "Superconducting energy gap and normal-state conductivity of a singledomain $\mathrm{YBa}_{2} \mathrm{Cu}_{3} \mathrm{O}_{7}$ crystal," Physical Review Letters, vol. 65, no. 6, pp. 801-804, 1990.

[96] O. V. Dolgov and M. L. Kulić, "Optical properties of strongly correlated systems with spin-density-wave order," Physical Review B, vol. 66, no. 13, Article ID 134510, 7 pages, 2002.

[97] D. B. Romero, C. D. Porter, D. B. Tanner, et al., "Quasiparticle damping in $\mathrm{Bi}_{2} \mathrm{Sr}_{2} \mathrm{CaCu}_{2} \mathrm{O}_{8}$ and $\mathrm{Bi}_{2} \mathrm{Sr}_{2} \mathrm{CuO}_{6}$," Physical Review Letters, vol. 68, no. 10, pp. 1590-1593, 1992.

[98] D. B. Romero, C. D. Porter, D. B. Tanner, et al., "On the phenomenology of the infrared properties of the copperoxide superconductors," Solid State Communications, vol. 82, no. 3, pp. 183-187, 1992.

[99] H. J. Kaufmann, Ph.D. thesis, University of Cambridge, Cambridge, UK, February 1999.

[100] A. V. Puchkov, D. N. Basov, and T. Timusk, "The pseudogap state in high- $T_{c}$ superconductors: an infrared study," Journal of Physics, vol. 8, no. 48, pp. 10049-10082, 1996.

[101] J. Hwang, T. Timusk, and G. D. Gu, "High-transitiontemperature superconductivity in the absence of the magnetic-resonance mode," Nature, vol. 427, no. 6976, pp. 714-717, 2004.

[102] M. Norman, "Shine a light," Nature, vol. 427, no. 6976, p. 692, 2004.

[103] F. Gao, D. B. Romero, D. B. Tanner, J. Talvacchio, and M. G. Forrester, "Infrared properties of epitaxial $\mathrm{La}_{2-x} \mathrm{Sr}_{x} \mathrm{CuO}_{4}$ thin films in the normal and superconducting states," Physical Review B, vol. 47, no. 2, pp. 1036-1052, 1993.

[104] A. V. Boris, N. N. Kovaleva, O. V. Dolgov, et al., "In-plane spectral weight shift of charge carriers in $\mathrm{YBa}_{2} \mathrm{Cu}_{3} \mathrm{O}_{6.9}$," Science, vol. 304, no. 5671, pp. 708-710, 2004.

[105] J. Schutzmann, B. Gorshunov, K. F. Renk, et al., "Far-infrared hopping conductivity in the $\mathrm{CuO}$ chains of a single-domain $\mathrm{YBa}_{2} \mathrm{Cu}_{3} \mathrm{O}_{7}$-crystal," Physical Review B, vol. 46, no. 1, pp. 512-515, 1992.

[106] K. Kamarãs, S. L. Herr, C. D. Porter, et al., "Erratumml: in a clean high- $T_{c}$ superconductor you do not see the gap (Physical Review Letters (1990) 64, 14 (1692))," Physical Review Letters, vol. 64, no. 14, p. 1692, 1990.
[107] B. Vignolle, S. M. Hayden, D. F. McMorrow, et al., "Two energy scales in the spin excitations of the high-temperature superconductor $\mathrm{La}_{2-x} \mathrm{Sr}_{x} \mathrm{CuO}_{4}$," Nature Physics, vol. 3, no. 3, pp. 163-167, 2007.

[108] I. Božović, J. H. Kim, J. S. Harris Jr., C. B. Eom, J. M. Phillips, and J. T. Cheung, "Reflectance and Raman spectra of metallic oxides, $\mathrm{LaSrCoO}$ and $\mathrm{CaSrRuO}$ : resemblance to superconducting cuprates," Physical Review Letters, vol. 73, no. 10, pp. 1436-1439, 1995.

[109] J. E. Hirsch, "Superconductors that change color when they become superconducting," Physica C, vol. 201, no. 3-4, pp. 347-361, 1992.

[110] P. F. Maldague, "Optical spectrum of a Hubbard chain," Physical Review B, vol. 16, no. 6, pp. 2437-2446, 1977.

[111] H. J. A. Molegraaf, C. Presura, D. van der Marel, P. H. Kes, and M. Li, "Superconductivity-induced transfer of in-plane spectral weight in $\mathrm{Bi}_{2} \mathrm{Sr}_{2} \mathrm{CaCu}_{2} \mathrm{O}_{8+\delta}$," Science, vol. 295, no. 5563, pp. 2239-2241, 2002.

[112] R. Beck, Y. Dagan, A. Milner, et al., "Transition in the tunneling conductance of $\mathrm{YBa}_{2} \mathrm{Cu}_{3} \mathrm{O}_{7-\delta}$ films in magnetic fields up to 32.4T," Physical Review B, vol. 72, no. 10, Article ID 104505, 4 pages, 2005.

[113] F. Carbone, A. B. Kuzmenko, H. J. A. Molegraaf, E. van Heumen, E. Giannini, and D. van der Marel, "Inplane optical spectral weight transfer in optimally doped $\mathrm{Bi}_{2} \mathrm{Sr}_{2} \mathrm{Ca}_{2} \mathrm{Cu}_{3} \mathrm{O}_{10}$," Physical Review B, vol. 74, no. 2, Article ID 024502, 2006.

[114] F. Carbone, A. B. Kuzmenko, H. J. A. Molegraaf, et al., "Doping dependence of the redistribution of optical spectral weight in $\mathrm{Bi}_{2} \mathrm{Sr}_{2} \mathrm{CaCu}_{2} \mathrm{O}_{8+\delta}$," Physical Review B, vol. 74, no. 6, Article ID 064510, 2006.

[115] A. F. Santander-Syro, et al., Europhysics Letters, vol. 51, p. 16380, 1995.

[116] L. Vidmar, J. Bonča, S. Maekawa, and T. Tohyama, "Bipolaron in the t-J model coupled to longitudinal and transverse quantum lattice vibrations," Physical Review Letters, vol. 103, no. 18, Article ID 186401, 2009.

[117] G. Deutscher, A. F. Santander-Syro, and N. Bontemps, "Kinetic energy change with doping upon superfluid condensation in high-temperature superconductors," Physical Review B, vol. 72, no. 9, Article ID 092504, 3 pages, 2005.

[118] H. J. Kaufmann, E. G. Maksimov, and E. K. H. Salje, "Electron-phonon interaction and optical spectra of metals," Journal of Superconductivity and Novel Magnetism, vol. 11, no. 6, pp. 755-768, 1998.

[119] T. A. Friedman, et al., "Direct measurement of the anisotropy of the resistivity in the $a-b$ plane of twin-free, single-crystal, superconducting $\mathrm{YBa}_{2} \mathrm{Cu}_{3} \mathrm{O}_{7-\delta}$," Physical Review B, vol. 42, pp. 6217-6221, 1990.

[120] P. B. Allen, "Is kinky conventional?" Nature, vol. 412, no. 6846, pp. 494-495, 2001.

[121] I. I. Mazin and O. V. Dolgov, "Estimation of the electronphonon coupling in $\mathrm{YBa}_{2} \mathrm{Cu}_{3} \mathrm{O}_{7}$ from the resistivity," Physical Review B, vol. 45, no. 5, pp. 2509-2511, 1992.

[122] T. Kondo, T. Takeuchi, S. Tsuda, and S. Shin, "Electrical resistivity and scattering processes in $(\mathrm{Bi}, \mathrm{Pb})_{2}(\mathrm{Sr}, \mathrm{La}) 2 \mathrm{CuO}_{6+\delta}$ studied by angle-resolved photoemission spectroscopy," Physical Review B, vol. 74, no. 22, Article ID 224511, 2006.

[123] J. Meng, G. Liu, W. Zhang, et al., "Growth, characterization and physical properties of high-quality large single crystals of $\mathrm{Bi}_{2}\left(\mathrm{Sr}_{2-x} \mathrm{La}_{x}\right) \mathrm{CuO}_{6+\delta}$ high-temperature superconductors," Superconductor Science and Technology, vol. 22, no. 4, Article ID 045010, 2009. 
[124] W. E. Pickett, "Temperature-dependent resistivity from phonons in cuprate superconductors," Journal of Superconductivity, vol. 4, no. 6, pp. 397-407, 1991.

[125] D. M. King, Z.-X. Shen, D. S. Dessau, et al., "Observation of a saddle-point singularity in $\mathrm{Bi}_{2}\left(\mathrm{Sr}_{0.97} \mathrm{Pr}_{0.03}\right) 2 \mathrm{CuO}_{6+\delta}$ and its implications for normal and superconducting state properties," Physical Review Letters, vol. 73, no. 24, pp. 32983301, 1994.

[126] S. Martin, A. T. Fiory, R. M. Fleming, L. F. Schneemeyer, and J. V. Waszczak, "Normal-state transport properties of $\mathrm{Bi}_{2+x} \mathrm{Sr}_{2-y} \mathrm{CuO}_{6+\delta}$ crystals," Physical Review B, vol. 41, no. 1, pp. 846-849, 1990.

[127] S. I. Vedeneev, A. G. M. Jansen, and P. Wyder, "Magnetotransport and magnetotunneling in single-layer, twolayer, and three-layer $\mathrm{Bi}_{2} \mathrm{Sr}_{2} \mathrm{Ca}_{n-1} \mathrm{Cu}_{n} \mathrm{O}_{z}(n=1,2,3)$ single crystals," Physica B, vol. 300, no. 1-4, pp. 38-51, 2001.

[128] G. Varelogiannis and E. N. Economou, "Small-q electronphonon scattering and linear dc resistivity in high- $T_{c}$ oxides," Europhysics Letters, vol. 42, no. 3, pp. 313-318, 1998.

[129] M. L. Kulić and O. V. Dolgov, "Forward electron-phonon scattering and HTSC," in High Temperature Superconductivity, S. Barnes, J. Ashkenazi, J. Cohn, and F. Zuo, Eds., vol. 483 of AIP Conference Proceedings, p. 63, 1999.

[130] R. Zeyher and M. L. Kulić, "Renormalization of the electronphonon interaction by strong electronic correlations in high$T_{c}$ superconductors," Physical Review B, vol. 53, no. 5, pp. 2850-2862, 1996.

[131] D. Mihailovic and V. V. Kabanov, "Dynamic inhomogeneity, pairing and superconductivity in cuprates," Structure and Bonding, vol. 114, pp. 331-364, 2005.

[132] V. V. Kabanov, J. Demsar, and D. Mihailovic, "Kinetics of a superconductor excited with a femtosecond optical pulse," Physical Review Letters, vol. 95, no. 14, Article ID 147002, 4 pages, 2005.

[133] A. Bansil and M. Lindroos, "Importance of matrix elements in the ARPES spectra of BISCO," Physical Review Letters, vol. 83, no. 24, pp. 5154-5157, 1999.

[134] T. Cuk, F. Baumberger, D. H. Lu, et al., "Coupling of the B1g Phonon to the antinodal electronic states of $\mathrm{Bi}_{2} \mathrm{Sr}_{2} \mathrm{Ca}_{0.92} \mathrm{Y}_{0.08} \mathrm{Cu}_{2} \mathrm{O}_{8+\delta}$," Physical Review Letters, vol. 93, no. 11, Article ID 117003, 2004.

[135] X. J. Zhou, J. Shi, T. Yoshida, et al., "Multiple bosonic mode coupling in the electron self-energy of $\left(\mathrm{La}_{2-x} \mathrm{Sr}_{x}\right) \mathrm{CuO}_{4}$," Physical Review Letters, vol. 95, no. 11, Article ID 117001, 4 pages, 2005.

[136] T. Valla, T. E. Kidd, W.-G. Yin, et al., "High-energy kink observed in the electron dispersion of high-temperature cuprate superconductors," Physical Review Letters, vol. 98, no. 16, Article ID 167003, 2007.

[137] M. L. Kulić and O. V. Dolgov, "Angle-resolved photoemission spectra of $\mathrm{Bi}_{2} \mathrm{Sr}_{2} \mathrm{CaCu}_{2} \mathrm{O}_{8}$ show a Coulomb coupling $\approx 1$ and an electron-phonon coupling of 2-3," Physical Review B, vol. 76, no. 13, Article ID 132511, 2007.

[138] T. Cuk, D. H. Lu, X. J. Zhou, Z.-X. Shen, T. P. Devereaux, and N. Nagaosa, "A review of electron-phonon coupling seen in the high- $T_{c}$. Superconductors by angle-resolved photoemission studies (ARPES)," Physica Status Solidi (B), vol. 242, no. 1, pp. 11-29, 2005.

[139] G.-H. Gweon, T. Sasagawa, S. Y. Zhou, et al., "An unusual isotope effect in a high-transition-temperature superconductor," Nature, vol. 430, no. 6996, pp. 187-190, 2004.

[140] E. G. Maksimov, O. V. Dolgov, and M. L. Kulić, "Electronphonon interaction with the forward scattering peak and the angle-resolved photoemission spectra isotope shift in $\mathrm{Bi}_{2} \mathrm{Sr}_{2} \mathrm{CaCu}_{2} \mathrm{O}_{8}$," Physical Review B, vol. 72, no. 21, Article ID 212505, 4 pages, 2005.

[141] J. F. Douglas, H. Iwasawa, Z. Sun, et al., "Superconductors: unusual oxygen isotope effects in cuprates?" Nature, vol. 446, no. 7133, article E5, 2007.

[142] H. Iwasawa, Y. Aiura, T. Saitoh, et al., "A re-examination of the oxygen isotope effect in ARPES spectra of Bi2212," Physica C, vol. 463-465, pp. 52-55, 2007.

[143] K. M. Shen, F. Ronning, D. E. Lu, et al., "Missing quasiparticles and the chemical potential puzzle in the doping evolution of the cuprate superconductors," Physical Review Letters, vol. 93, no. 26, Article ID 267002, 2004.

[144] O. Rösch, O. Gunnarsson, X. J. Zhou, et al., "Polaronic behavior of undoped high- $T_{c}$ cuprate superconductors from angleresolved photoemission spectra," Physical Review Letters, vol. 95, no. 22, Article ID 227002, 4 pages, 2005.

[145] A. S. Mishchenko and N. Nagaosa, "Electron-phonon coupling and a polaron in the t-J model: from the weak to the strong coupling regime," Physical Review Letters, vol. 93, no. 3, Article ID 036402, 2004.

[146] S. Ciuchi, F. De Pasquale, S. Fratini, and D. Feinberg, "Dynamical mean-field theory of the small polaron," Physical Review B, vol. 56, no. 8, pp. 4494-4512, 1997.

[147] M. Tsunekawa, A. Sekiyama, S. Kasai, et al., "Bulk electronic structures and strong electron-phonon interactions in an electron-doped high-temperature superconductor," New Journal of Physics, vol. 10, Article ID 073005, 2008.

[148] S. R. Park, D. J. Song, C. S. Leem, et al., "Angle-resolved photoemission spectroscopy of electron-doped cuprate superconductors: isotropic electron-phonon coupling," Physical Review Letters, vol. 101, no. 11, Article ID 117006, 2008.

[149] J. C. Campuzano, H. Ding, M. R. Norman, et al., "Direct observation of particle-hole mixing in the superconducting state by angle-resolved photoemission," Physical Review B, vol. 53, no. 22, pp. R14737-R14740, 1996.

[150] H. Matsui, T. Sato, T. Takahashi, et al., "BCS-like Bogoliubov quasiparticles in high- $T_{c}$ superconductors observed by angleresolved photoemission spectroscopy," Physical Review Letters, vol. 90, no. 21, Article ID 217002, 4 pages, 2003.

[151] M. L. Kulić and O. V. Dolgov, "Dominance of the electronphonon interaction with forward scattering peak in high$T_{c}$ superconductors: theoretical explanation of the ARPES kink," Physical Review B, vol. 71, no. 9, Article ID 092505, 4 pages, 2005.

[152] Y. Chen, A. Iyo, W. Yang, et al., "Anomalous Fermi-surface dependent pairing in a self-doped high- $T_{c}$ superconductor," Physical Review Letters, vol. 97, no. 23, Article ID 236401, 2006.

[153] W. Xie, O. Jepsen, O. K. Andersen, Y. Chen, and Z.-X. Shen, "Insights from angle-resolved photoemission spectroscopy of an undoped four-layered two-gap high- $T_{c}$ superconductor," Physical Review Letters, vol. 98, no. 4, Article ID 047001, 2007.

[154] L. Zhu, P. J. Hirschfeld, and D. J. Scalapino, "Elastic forward scattering in the cuprate superconducting state," Physical Review B, vol. 70, no. 21, Article ID 214503, 13 pages, 2004.

[155] M. L. Kulić and V. Oudovenko, "Why is d-wave pairing in HTS robust in the presence of impurities?" Solid State Communications, vol. 104, no. 7, pp. 375-379, 1997.

[156] M. L. Kulić and O. V. Dolgov, "Anisotropic impurities in anisotropic superconductors," Physical Review B, vol. 60, no. 18, pp. 13062-13069, 1999. 
[157] H.-Y. Kee, "Effect of doping-induced disorder on the transition temperature in high- $T_{c}$ cuprates," Physical Review B, vol. 64, no. 1, Article ID 012506, 2001.

[158] J. Kirtley, "Tunneling measurements of the cuprate superconductors," in Handbook of High-Temperature Superconductivity: Theory and Experiment, J. R. Schrieffer, Ed., p. 19, Springer, Berlin, Germany, 2007.

[159] B. Renker, F. Gompf, D. Ewert, et al., "Changes in the phonon spectra of Bi 2212 superconductors connected with the metal-semiconductor transition in the series of $\mathrm{Bi}_{2} \mathrm{Sr}_{2}\left(\mathrm{Ca}_{1-x} \mathrm{Y}_{x}\right) \mathrm{Cu}_{2} \mathrm{O}_{8}$ compounds," Zeitschrift für Physik B, vol. 77, no. 1, pp. 65-68, 1989.

[160] G. Deutscher, N. Hass, Y. Yagil, A. Revcolevschi, and G. Dhalenne, "Evidence for strong electron-phonon interaction in point contact spectroscopy of superconducting oriented $\mathrm{La}_{1-x} \mathrm{Sr}_{x} \mathrm{CuO}_{4}$," Journal of Superconductivity, vol. 7, no. 2, pp. 371-374, 1994.

[161] Y. Sidis, S. Pailhès, B. Keimer, P. Bourges, C. Ulrich, and L. P. Regnault, "Magnetic resonant excitations in high- $T_{c}$ superconductors," Physica Status Solidi (B), vol. 241, no. 6, pp. 1204-1210, 2004.

[162] G.-M. Zhao, "Fine structure in the tunneling spectra of electron-doped cuprates: no coupling to the magnetic resonance mode," Physical Review Letters, vol. 103, no. 23, Article ID 236403, 2009.

[163] O. Fischer, M. Kugler, I. Maggio-Aprile, C. Berthod, and C. Renner, "Scanning tunneling spectroscopy of hightemperature superconductors," Reviews of Modern Physics, vol. 79, no. 1, pp. 353-419, 2007.

[164] O. K. Andersen, O. Jepsen, A. I. Liechtenstein, and I. I. Mazin, "Plane dimpling and saddle-point bifurcation in the band structures of optimally doped high-temperature superconductors: a tight-binding model," Physical Review B, vol. 49, no. 6, pp. 4145-4157, 1994.

[165] Q. Huang, J. F. Zasadzinski, N. Tralshawala, et al., "Tunnelling evidence for predominantly electronphonon coupling in superconducting $\mathrm{Ba}_{1-x} \mathrm{~K}_{x} \mathrm{BiO}_{3}$ and $\mathrm{Nd}_{2-x} \mathrm{Ce}_{x} \mathrm{CuO}_{4-y}$," Nature, vol. 347, no. 6291, pp. 369-372, 1990.

[166] P. Samuely, N. L. Bobrov, A. G. M. Jansen, P. Wyder, S. N. Barilo, and S. V. Shiryaev, "Tunneling measurements of the electron-phonon interaction in $\mathrm{Ba}_{1-x} \mathrm{~K}_{x} \mathrm{BiO}_{3}$," Physical Review B, vol. 48, no. 18, pp. 13904-13910, 1993.

[167] P. Samuely, P. Szabó, A. G. M. Jansen, et al., "From superconducting to normal density of states of $\mathrm{Ba}_{1-x} \mathrm{~K}_{x} \mathrm{BiO}_{3}$ by tunneling in high magnetic fields," Physica B, vol. 194196, pp. 1747-1748, 1994.

[168] O. Rösch and O. Gunnarsson, "Electron-phonon interaction in the t-J model," Physical Review Letters, vol. 92, no. 14, Article ID 146403, 2004.

[169] K. J. von Szczepanski and K. W. Becker, "Coupling of electrons and phonons in a doped antiferromagnet," Zeitschrift für Physik B, vol. 89, no. 3, pp. 327-334, 1992.

[170] D. Reznik, "Giant electron-phonon anomaly in doped $\mathrm{La}_{2} \mathrm{CuO}_{4}$ and other cuprates," Advances in Condensed Matter Physics, vol. 2010, Article ID 523549, 2010.

[171] G. Khaliullin and P. Horsch, "Theory of the density fluctuation spectrum of strongly correlated electrons," Physical Review B, vol. 54, no. 14, pp. R9600-R9603, 1996.

[172] L. Pintschovius, "Electron-phonon coupling effects explored by inelastic neutron scattering," Physica Status Solidi (B), vol. 242, no. 1, pp. 30-50, 2005.
[173] D. Reznik, L. Pintschovius, M. Ito, et al., "Electron-phonon coupling reflecting dynamic charge inhomogeneity in copper oxide superconductors," Nature, vol. 440, no. 7088, pp. 11701173, 2006.

[174] D. Reznik, L. Pintschovius, M. Fujita, K. Yamada, G. D. Gu, and J. M. Tranquada, "Electron-phonon anomaly related to charge stripes: static stripe phase versus optimally doped superconducting $\mathrm{La}_{1.85} \mathrm{Sr}_{0.15} \mathrm{CuO}_{4}$, Journal of Low Temperature Physics, vol. 147, no. 3-4, pp. 353-364, 2007.

[175] L. Pintschovius, D. Reznik, W. Reichardt, et al., "Oxygen phonon branches in $\mathrm{YBa}_{2} \mathrm{Cu}_{3} \mathrm{O}_{7}$," Physical Review B, vol. 69, no. 21, Article ID 214506, 2004.

[176] K.-P. Bohnen, R. Heid, and M. Krauss, "Phonon dispersion and electron-phonon interaction for $\mathrm{YBa}_{2} \mathrm{Cu}_{3} \mathrm{O}_{7}$ from firstprinciples calculations," Europhysics Letters, vol. 64, no. 1, pp. 104-110, 2003.

[177] T. Bauer and C. Falter, "The impact of dynamical screening on the phonon dynamics of LaCuO," Physical Review B, vol. 80, Article ID 094525, 2009.

[178] D. Reznik, G. Sangiovanni, O. Gunnarsson, and T. P. Devereaux, "Photoemission kinks and phonons in cuprates," Nature, vol. 455, no. 7213, pp. E6-E7, 2008.

[179] R. Zeyher and M. L. Kulić, "Statics and dynamics of charge fluctuations in the t-J model," Physical Review B, vol. 54, no. 13, pp. 8985-8988, 1996.

[180] M. L. Kulić and R. Zeyher, "Novel 1/N expansion for selfenergy and correlation functions of the Hubbard model," Modern Physics Letters B, vol. 11, no. 8, pp. 333-338, 1997.

[181] M. L. Kulić and G. Akpojotor, in preparation.

[182] Z. B. Huang, W. Hanke, E. Arrigoni, and D. J. Scalapino, "Electron-phonon vertex in the two-dimensional one-band Hubbard model," Physical Review B, vol. 68, no. 22, Article ID 220507, 4 pages, 2003.

[183] E. Cappelluti, B. Cerruti, and L. Pietronero, "Charge fluctuations and electron-phonon interaction in the finite- $U$ Hubbard model," Physical Review B, vol. 69, no. 16, Article ID 161101, 2004.

[184] C. Thomsen, M. Cardona, B. Gegenheimer, R. Liu, and A. Simon, "Untwinned single crystals of $\mathrm{YBa}_{2} \mathrm{Cu}_{3} \mathrm{O}_{7}$ : an optical investigation of the $a-b$ anisotropy," Physical Review B, vol. 37, no. 16, pp. 9860-9863, 1988.

[185] C. Thomsen and M. Cardona, in Physical Properties of High Temperature Superconductors I, D. M. Ginzberg, Ed., p. 409, World Scientific, Singapore, 1989.

[186] R. Feile, "Lattice vibrations in high- $T_{c}$ superconductors: optical spectroscopy and lattice dynamics," Physica $C$, vol. 159, no. 1-2, pp. 1-32, 1989.

[187] C. Thomsen, "Light scattering in high- $T_{c}$-superconductors," in Light Scattering in Solids VI, M. Cardona and G. Guentherodt, Eds., p. 285, Springer, Berlin, Germany, 1991.

[188] V. G. Hadjiev, X. Zhou, T. Strohm, M. Cardona, Q. M. Lin, and C. W. Chu, "Strong superconductivity-induced phonon self-energy effects in $\mathrm{HgBa}_{2} \mathrm{Ca}_{3} \mathrm{Cu}_{4} \mathrm{O}_{10+\delta}$," Physical Review B, vol. 58, no. 2, pp. 1043-1050, 1998.

[189] S. N. Rashkeev and G. Wendin, "Electronic Raman continuum for $\mathrm{YBa}_{2} \mathrm{Cu}_{3} \mathrm{O}_{7-\delta}$ : effects of inelastic scattering and interband transitions," Physical Review B, vol. 47, no. 17, pp. 11603-11606, 1993.

[190] J. F. Franck, in Physical Properties of High Temperature Supercoductors V, D. M. Ginsberg, Ed., World Scientific, Singapore, 1994. 
[191] J. P. Franck, "Experimental studies of the isotope effect in high $T_{c}$ superconductors," Physica C, vol. 282-287, pp. 198201, 1997.

[192] H. Iwasawa, J. F. Douglas, K. Sato, et al., "An isotopic fingerprint of electron-phonon coupling in high- $T_{c}$ cuprates," Physical Review Letters, vol. 101, Article ID 157005, 2008.

[193] D. J. Scalapino, "The Electron-phonon interaction and strong-coupling superconductors," in Superconductivity, R. D. Parks, Ed., vol. 1, chapter 11, Dekker, New York, NY, USA, 1969.

[194] D. Rainer, "Principles of AB initio calculations of superconducting transition temperatures," in Progress in LowTemperature Physics, D. F. Brewer, Ed., p. 371, Elsevier, Amsterdam, The Netherlands, 1986.

[195] H. Krakauer, W. E. Pickett, and R. E. Cohen, "Large calculated electron-phonon interactions in $\mathrm{La}_{2-x} \mathrm{M}_{x} \mathrm{CuO}_{4}$," Physical Review B, vol. 47, no. 2, pp. 1002-1015, 1993.

[196] C. Falter, M. Klenner, G. A. Hoffmann, and Q. Chen, "Origin of phonon anomalies in $\mathrm{La}_{2} \mathrm{CuO}_{4}$," Physical Review B, vol. 55, no. 5, pp. 3308-3313, 1997.

[197] C. Falter, M. Klenner, and G. A. Hoffmann, "Screening and phonon-plasmon scenario as calculated from a realistic electronic bandstructure based on LDA for $\mathrm{La}_{2} \mathrm{CuO}_{4}$," Physica Status Solidi (B), vol. 209, no. 2, pp. 235-266, 1998.

[198] C. Falter, M. Klenner, and G. A. Hoffmann, "Anisotropy dependence of the c-axis phonon dispersion in the hightemperature superconductors," Physical Review B, vol. 57, no. 22, pp. 14444-14451, 1998.

[199] I. I. Mazin, S. N. Rashkeev, and S. Y. Savrasov, "Nonspherical rigid-muffin-tin calculations of electron-phonon coupling in high- $T_{c}$ perovskites," Physical Review B, vol. 42, no. 1, pp. 366-370, 1990.

[200] L. F. Mattheiss, "Electronic band properties and superconductivity in $\mathrm{La}_{2-y} \mathrm{X}_{y} \mathrm{CuO}_{4}$," Physical Review Letters, vol. 58, no. 10 , pp. 1028-1030, 1987.

[201] T. Jarlborg, "Electron-phonon coupling and charge transfer in $\mathrm{YBa}_{2} \mathrm{Cu}_{3} \mathrm{O}_{7}$ from band theory," Solid State Communications, vol. 67, no. 3, pp. 297-300, 1988.

[202] T. Jarlborg, "Weak screening of high frequency phonons and superconductivity in $\mathrm{YBa}_{2} \mathrm{Cu}_{3} \mathrm{O}_{7}$," Solid State Communications, vol. 71, no. 8, pp. 669-671, 1989.

[203] S. Yu. Savrasov, "Linear response calculations of lattice dynamics using muffin-tin basis sets," Physical Review Letters, vol. 69, no. 19, pp. 2819-2822, 1992.

[204] S. Yu. Savrasov and D. Yu. Savrasov, "Full-potential linearmuffin-tin-orbital method for calculating total energies and forces," Physical Review B, vol. 46, no. 19, pp. 12181-12195, 1992.

[205] S. Y. Savrasov and O. K. Andersen, "Linear-response calculation of the electron-phonon coupling in doped $\mathrm{CaCuO}_{2}$," Physical Review Letters, vol. 77, no. 21, pp. 4430-4433, 1996.

[206] V. J. Emery, "Theory of high- $T_{c}$ superconductivity in oxides," Physical Review Letters, vol. 58, no. 26, pp. 2794-2797, 1987.

[207] W. E. Picket, "Electronic structure of the high-temperature oxide superconductors," Reviews of Modern Physics, vol. 61, p. $433,1989$.

[208] J. van den Brink, M. B. J. Meinders, J. Lorenzana, R. Eder, and G. A. Sawatzky, "New phases in an extended hubbard model explicitly including atomic polarizabilities," Physical Review Letters, vol. 75, no. 25, pp. 4658-4661, 1995.

[209] J. van den Brink, M. B. J. Meinders, J. Lorenzana, R. Eder, and G. A. Sawatzky, "New phases in an extended hubbard model explicitly including atomic probabilities," Physical Review Letters, vol. 76, p. 2826, 1996.
[210] J. van den Brink, thesis, University Groningen, 1997.

[211] H. Eskes and G. A. Sawatzky, "Doping dependence of highenergy spectral weights for the high- $T_{c}$ cuprates," Physical Review B, vol. 43, no. 1, pp. 119-129, 1991.

[212] F. C. Zhang and T. M. Rice, "Effective Hamiltonian for the superconducting Cu oxides," Physical Review B, vol. 37, no. 7, pp. 3759-3761, 1988.

[213] R. Hayn, V. Yushankhai, and S. Lovtsov, "Analysis of the singlet-triplet model for the copper oxide plane within the paramagnetic state," Physical Review B, vol. 47, no. 9, pp. 5253-5262, 1993.

[214] A. E. Ruckenstein and S. Schmitt-Rink, "New approach to strongly correlated systems: $1 \mathrm{~N}$ expansions without slave bosons," Physical Review B, vol. 38, no. 10, pp. 7188-7191, 1988.

[215] A. Greco and R. Zeyher, "Superconducting instabilities in the tt' hubbard model in the large-N limit," Europhysics Letters, vol. 35, no. 2, pp. 115-120, 1996.

[216] R. Zeyher and A. Greco, "Superconductivity in the t-J model in the large-N limit," Zeitschrift fur Physik B, vol. 104, no. 4, pp. 737-740, 1997.

[217] M. Grilli and G. Kotliar, "Fermi-liquid parameters and superconducting instabilities of a generalized t-J model," Physical Review Letters, vol. 64, no. 10, pp. 1170-1173, 1990.

[218] P. Fulde, J. Keller, and G. Zwicknagel, "Theory of heavy fermion systems," in Solid State Physics, H. Ehrenreich and D. Turnbul, Eds., vol. 41, p. 2, Academic Press, 1988.

[219] M. Grilli and C. Castellani, "Electron-phonon interactions in the presence of strong correlations," Physical Review B, vol. 50, no. 23, pp. 16880-16898, 1994.

[220] C. Grimaldi, L. Pietronero, and S. Străssler, "Nonadiabatic superconductivity. I. Vertex corrections for the electronphonon interactions," Physical Review B, vol. 52, no. 14, pp. 10516-10529, 1995.

[221] C. Grimaldi, L. Pietronero, and S. Străssler, "Nonadiabatic superconductivity. II. Generalized Eliashberg equations beyond Migdal's theorem," Physical Review B, vol. 52, no. 14, pp. 10530-10546, 1995.

[222] G. Kotliar and J. Liu, "Superconducting instabilities in the large-U limit of a generalized hubbard model," Physical Review Letters, vol. 61, no. 15, pp. 1784-1787, 1988.

[223] J. H. Kim and Z. Teanović, "Effects of strong Coulomb correlations on the phonon-mediated superconductivity: a model inspired by copper oxides," Physical Review Letters, vol. 71, no. 25, pp. 4218-4221, 1993.

[224] S. Ishihara and N. Nagaosa, "Interplay of electron-phonon interaction and electron correlation in high-temperature superconductivity," Physical Review B, vol. 69, no. 14, Article ID 144520, 2004.

[225] O. V. Dolgov and E. G. Maksimov, "Critical temperature of superconductors with a strong coupling," Uspekhi Fizicheskikh Nauk, vol. 138, p. 95, 1982.

[226] O. V. Dolgov and E. G. Maksimov, "Electron-phonon interaction and superconductivity," in Thermodynamics and Electrodynamics of Superconductors, V. L. Ginzburg, Ed., chapter 1, Nova Science, Hauppauge, NY, USA, 1987.

[227] F. Marsiglio and J. P. Carbotte, "Electron-phonon superconductivity," in Superconductivity I, J. Kettersson and K. H. Bennemann, Eds., Springer, Berlin, Germany, 2008.

[228] W. L. McMillan and J. M. Rowell, "Lead phonon spectrum calculated from superconducting density of states," Physical Review Letters, vol. 14, no. 4, pp. 108-112, 1965.

[229] A. A. Galkin, A. I. Dyashenko, and V. M. Svistunov, "Determination of the energy gap parameter and the 
electron-phonon interaction function in superconductors on basis tunnel data," Zhurnal Eksperimental'noi i Teoreticheskoi Fiziki, vol. 66, p. 2262, 1974.

[230] V. M. Svistunov, A. I. D’yachenko, and M. A. Belogolovskii, "Elastic tunneling spectroscopy of single-particle excitations in metals," Journal of Low Temperature Physics, vol. 31, no. 3-4, pp. 339-356, 1978.

[231] Yu. M. Ivanshenko and Yu. V. Medvedev, "Many-body and inelastic tunnel spectroscopy of quasi-particle excitations in normal metals," Fizika Nizkikh Temperatur, vol. 2, p. 143, 1976.

[232] D. J. Scalapino, J. R. Schrieffer, and J. W. Wilkins, "Strongcoupling superconductivity. I," Physical Review, vol. 148, no. 1, pp. 263-279, 1966.

[233] J. R. Schrieffer, Theory of Superconductivity, W. A. Benjamin, New York, NY, USA, 1964. 

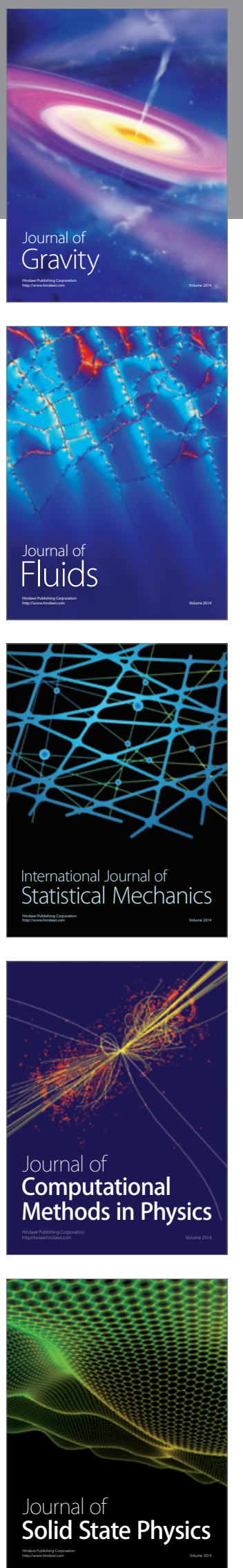

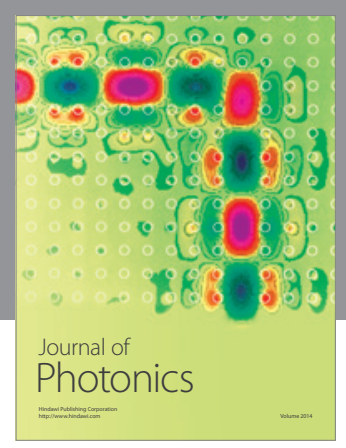

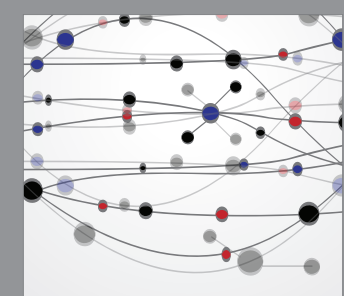

The Scientific World Journal
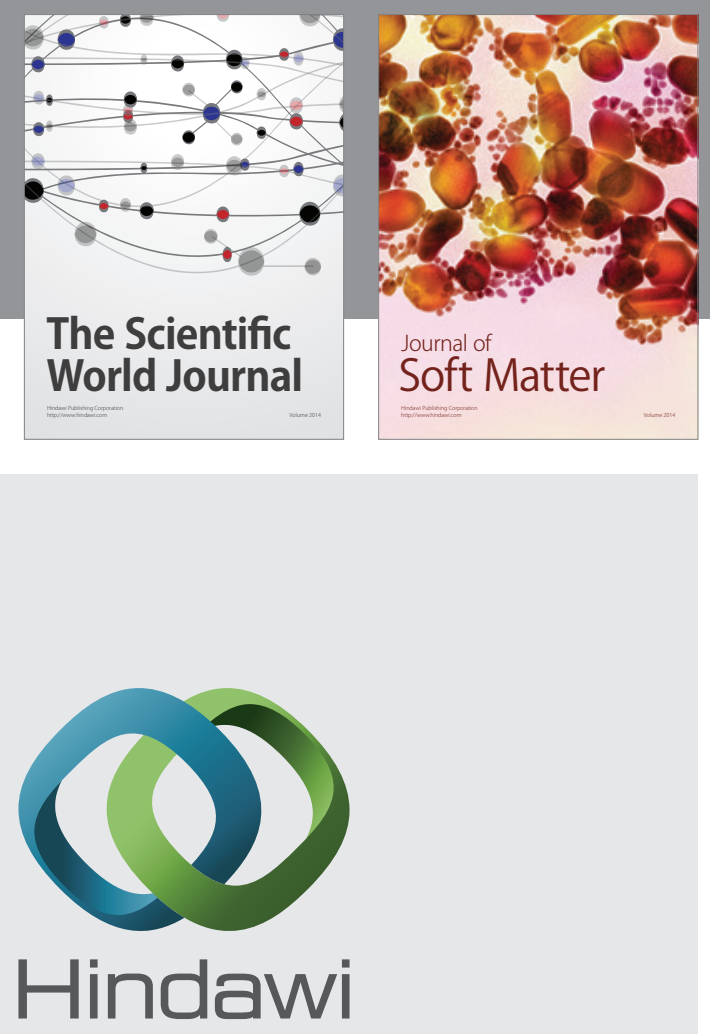

Submit your manuscripts at

http://www.hindawi.com
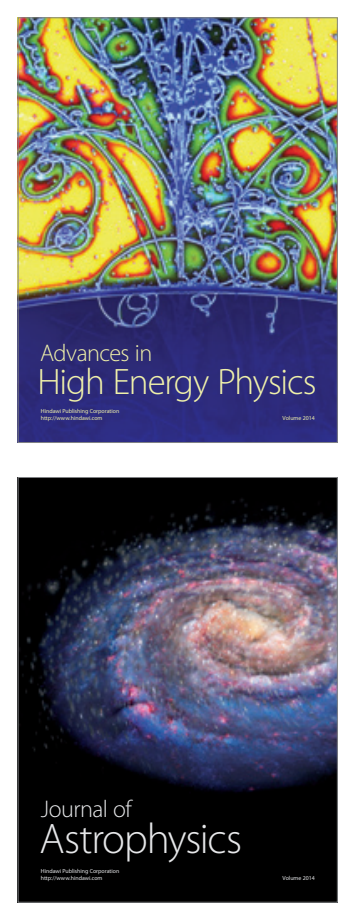
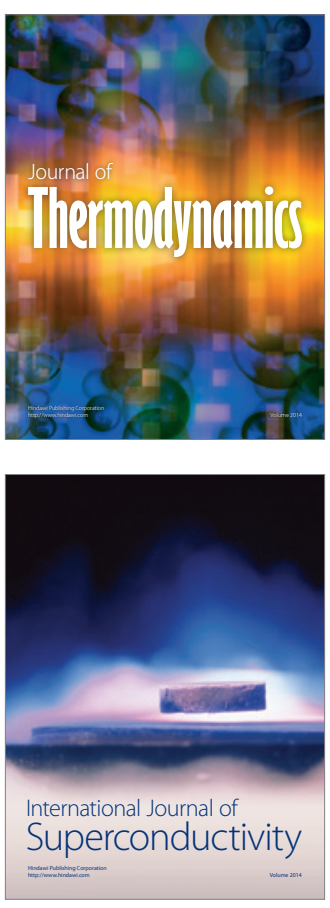
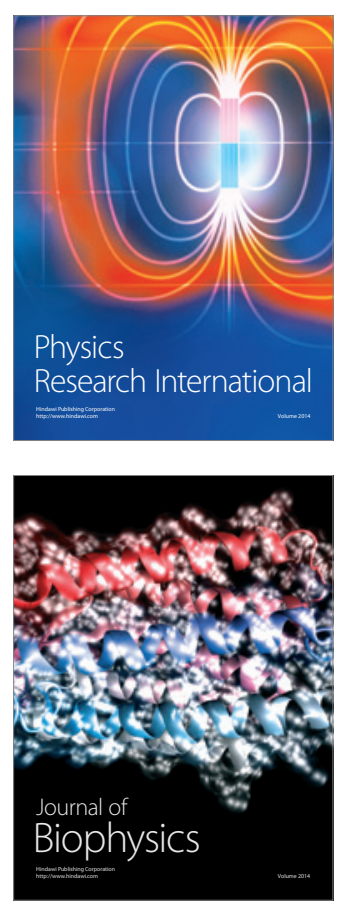
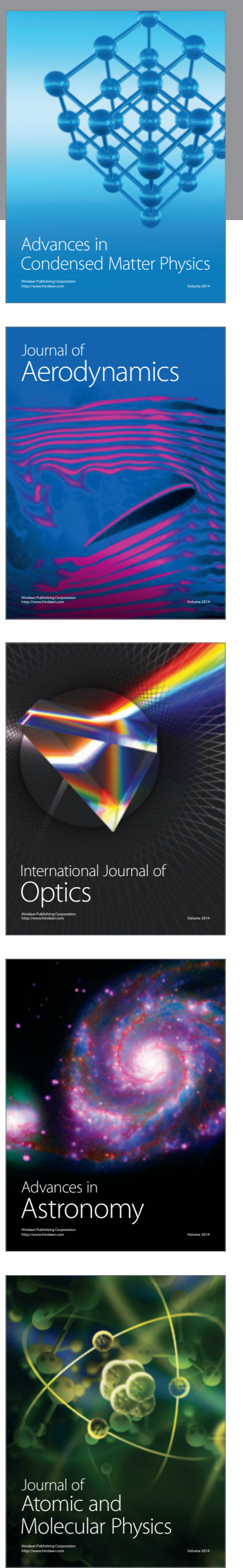